\title{
Versuche über Längsschub, Querbiegung und \\ Quervorspannung in Zugplatten von Betonträgern
}

\section{Working Paper}

Author(s):

Bacchetta, Aldo; Bachmann, Hugo

Publication date:

1979

Permanent link:

https://doi.org/10.3929/ethz-a-000184909

Rights / license:

In Copyright - Non-Commercial Use Permitted

Originally published in:

Bericht / Institut für Baustatik und Konstruktion ETH Zürich 6504(10) 
Versuche über Längsschub, Querbiegung und Quervorspannung in Zugplatten von Betonträgern

Aldo Bacchetta

Hugo Bachmann 
CIP-Kurztitelaufnahme der Deutschen Bibliothek

\section{Bacchetta, Aldo:}

Versuche über Längsschub, Querbiegung und Quervorspannung in Zugplatten von Betonträgern/ von Aldo Bacchetta; Hugo Bachmann. -Basel, Boston, Stuttgart : Birkhäuser, 1979.

(Bericht/Institut für Baustatik und Konstruktion ETH Zürich ; Nr. 6504-10)

ISBN 3-7643-1153-3

NE: Bachmann, Hugo:

Nachdruck verboten.

Alle Rechte, insbesondere das der Übersetzung in fremde Sprachen und der Reproduktion auf photostatischem Wege oder durch Mikrofilm,

vorbehalten.

(C) Birkhäuser Verlag Basel, 1979

ISBN 3-7643-1153-3 
Versuche über Längsschub, Querbiegung

und Quervorspannung in Zugplatten von Betonträgern

\author{
von \\ Dipl. Ing. Aldo Bacchetta \\ Prof. Dr. Hugo Bachmann \\ Institut für Baustatik und Konstruktion \\ Eidgenössische Technische Hochschule Zürich
}


1. EINLEITUNG

\section{1}

1.1 Allgemeines 1

1.2 Zielsetzung 1

1.3 Bemessungsmodelle . 2

1.3.1 Längsschub allein 2

1.3 .2 Längsschub mit Querbiegung 3

1.3.3 Längsschub mit Querbiegung und Quervorspannung 3

1.4 Versuchsplanung 4

2. VERSUCHSTRAEGER 7

2.1 Beschreibung 7

2.1.1 Abmessungen, Armierungen . 7

2.1 .2 Herstellung, Vorspannung, Lagerung 7

2.2 Baustoffe B

2.2.1 Armierungsstahl, Spannstahl 8

2.2 .2 Beton 8

2.2 .3 Zementmörtel o . 8

2.3 Bemessung und theoretische werte 9

2.3.1 Berechnete Lasten und Schubspannungen $g$

2.3.2 Längsbiegearmierung 9

2.3 .3 Schubarmierung im Steg 10

2.3.4 Querarmierung in der Oruckplatte 10

2.3.5 Querarmierung in der Zugplatte 10

3. VERSUCHSDURCHFUEHRUNG 12

3.1 Versuchsanlage 12

3.2 Versuchsablauf 12

3.3 Messungen 13

3.3.1 Lasten 13

3.3 .2 Verformungen 13

3.3 .3 Risse 14

4. VERSUCHSRESULTATE 15

4.1 Allgemeines 15

4.2 Biegeverhalten der Träger 16

4.2 .1 Durchbiegungen 16

4.2.2 Stauchungen der Druckplatte 16

4.2.3 Dehnungen der Längsbiegearmierung 16

4.2.4 Dehnungen der Längsbiegearmierung beim Träger Z2

4.3 Dehnungen der Plattenquerarmierung 17

4.3.1 Träger Z1 und Z2 (Längschub allein) 17

4.3.2 Träger Z4 (Längsschub allein, mit Querbiegung/ und Quervorspannung 18

4.3.3 Träger 23,25 und Z6 (Längsschub mit Querbiegung/ und Quervorspannung 19

4.4 Kräfte in der Plattenquerarmierung 21

4.4.1 Träger Z1 und Z2 (Längsschub allein) 21

4.4.2 Träger 24 (Längsschub allein, mit Querbiegung/ und Quervorspannung 21

4.4.3 Träger Z3, 25 und Z6 (Längsschub mit Querbiegung/ und Quervorspannung 22

4.5 Verlagerung des Angriffspunktes der Querzugkraft aus Längsschub 24

4.5.1 Längsschub allein, gleiche obere und untere Querarmierung 25

4.5.2 Längsschub allein, ungleiche obere und untere Querarmierung 25 
4.5.3 Längsschub mit Querbiegung, gleiche oder ungleiche abere und untere Armierung

4.6 Rissverhalten

4.6.1 Träger Z1. und $Z 2 \quad 25$

4.6.2 Träger $Z 4 \quad 26$

4.6.3 Träger $Z 3, Z 5$ und $Z 6 \quad 26$

4.7 Bruchverhalten $\quad 27$

4.7.1 Träger $Z 1$ und $Z 2 \quad 27$

4.7 .2 Träger $Z 4 \quad 27$

$\begin{array}{ll}4.7 .3 \text { Träger } Z 3, Z 5 \text { und } Z 6 & 27\end{array}$

5. FOLGERUNGEN $\quad 29$

$\begin{array}{ll}5.1 \text { Längsschub allein } & 29\end{array}$

5.1 .1 Tragfähigkeit $\quad 29$

5.1 .2 Verhalten im Gebrauchszustand 30

5.2 Längsschub mit Querbiegung 30

5.2.1 Tragfähigkeit 30

5.2.2 Verhalten im Gebrauchszustand 30

5.3 Längsschub mit Querbiegung und Quervorspannung 30

5.3.1 Tragfähigkeit 30

5.3.2 Verhalten im Gebrauchszustand 31

ZUSAMMENFASSUNG UND EMPFEHLUNGEN $\quad 32$

RESUME ET RECOMMANDATIONS $\quad 34$

$\begin{array}{lr}\text { SUMMARY AND RECOMMENDATIONS } & 37\end{array}$

$\begin{array}{ll}\text { VERDANKUNGEN } & 40\end{array}$

LITERATURVERZEICHNIS $\quad 41$

BEZEICHNUNGEN $\quad 42$

$\begin{array}{lr}\text { BILDER } & 45\end{array}$

ANHANG $\quad$ A1 


\section{EINLEITUNG}

1.1 Allgemeines

In Druck- bzw. Zugflanschen dünnwandiger Plattenbalken- und Hohlkastenquerschnitte treten ausser Längsdruck bzw. Längszug häufig auch Längsschub und Querbiegung auf.

Die Problematik in Druckflanschen wurde in [1] und [2] sowie in [3] und [4] eingehend untersucht und behandelt.

Die Problematik in Zugflanschen ist Gegenstand des vorliegenden Versuchsberichtes. Es sollen die Grundlagen für die Entwicklung einfacher, mit der Wirklichkeit gut übereinstimmender Bemessungsmodelle bereitgestellt werden.

An einem Element des zum Beispiel als Kragarm gelagerten Plattenbalkens mit ausserhalb des Steges eingelegter Längsbiegearmierung gemäss Bild 1 kännen in einem Schnitt I-I, je nach der äusseren Belastung, im Rissezustand folgende Schnittgrässen auftreten:

\section{a) Längsschub allein}

Wirkt eine Belastung $P$ in der Symmetrieebene des I-Trägers, so entsteht eine Längsschubkraft $\tau_{y x} \cdot d$.

\section{b) Längsschub mit Querbiegung}

Wirkt ferner eine Belastung $\bar{P}$ ausserhalb des Steges, so entsteht zum Längsschub hinzu ein Querbiegemoment $\bar{M}$. Die ebenfalls auftretende vertikale Schubkraft $\tau_{x y} \cdot d$ ist meist klein und wird in der vorliegenden Untersuchung nicht weiter berücksichtigt.

\section{c) Längsschub mit Querbiegung und Quervorspannung}

Ist die Zugplatte in Querrichtung vorgespannt, so wirkt ausser Längsschub und Querbiegung zusätzlich noch die Vorspannkraft $V$.

Zu diesen Problemkreisen wurden in den Jahren 1977 und 1978 am Institut für Baustatik und Konstruktion der ETH Zürich Versuche an insgesamt sechs Versuchsträgern durchgeführt. Zum Problemkreis "Längsschub allein" wurden zwei Versuchsträger und zu "Längsschub mit Querbiegung" sowie "Längsschub mit Querbiegung und Quervorspannung" vier Versuchsträger geprüft.

\subsection{Zielsetzung}

Die Ziele der hier beschriebenen Versuche waren:

- die Erforschung des Verformungs- und Tragverhaltens von Zugplatten von Betonträgern unter Längsschub, Querbiegung und Quervorspannung, und

- das Entwickeln und Ueberprüfen neuer Bemessungsmodelle anhand der Versuchsresultate, wobei zwei wesentliche Anforderungen an ein Modell gestellt wurden:

1. Das Modell soll möglichst gut mit der Wirklichkeit übereinstimmen.

2. Das Modell soll zu einem möglichst geringen Rechenaufwand führen. 


\subsubsection{Längsschub allein}

Im Falle von Längsschub werden in der Praxis die Beanspruchungen im Anschlussbereich Zugplatte - Steg normalerweise nach dem klassischen Modell (Hauptzugsspannungs-Modell) ermittelt. Wie sich bereits bei den Druckflansch-Versuchen herausgestellt hat, stimmt dieses Modell im Risszustand mit der Wirklichkeit schlecht überein.

Zweckmässiger ist beim Zugflansch ein Flanschfachwerk-Modell, ähnlich demjenigen für den Druckflansch ([1] und [2]). Die Grundidee dieses Flanschfachwerk-Modells vermittelt Bild 2a. Wird die Wirkung des Trägersteges durch ein Fachwerk mit der Höhe des inneren Hebelarms $z$ und der Diagonalenneigung $\alpha$ erfasst, so greifen an der Platte im Abstand $z \cdot \cot \alpha$ die horizontalen Komponenten der Stegdiagonalenkräfte $D$ an, das heisst $D \cdot \cos \alpha=Q \cdot \cot \alpha$. Diese müssen sich im Risszustand bei ausserhalb des Steges liegender Längsbiegearmierung in den Flanschen ausbreiten. Ersetzt man die in den beiden Flanschhälften liegende Längsbiegearmierung je in ihrem Schwerpunkt durch einen Zugstab, und ergänzt man die beiden Zugstäbe durch Stabdreiecke zur Kraftausbreitung, so entsteht ein einfaches Flanschfachwerk. Im Abstand $z \cdot \cot \alpha$ ergeben sich Querzugkräfte

$$
Z_{1}=\frac{Q}{2} \cdot \cot \alpha \cdot \tan \beta
$$

welche die Zugplatte im Anschluss Platte - Steg auf Querzug beanspruchen.

Wird dieses diskrete Fachwerk "verschmiert", so beträgt die Querzugkraft $Z$ pro Längeneinheit

$$
Z=\frac{Q}{2 \cdot z} \cdot \tan \beta
$$

Das Versatzmass $v$ der Querarmierung, bezogen auf den Trägerquerschnitt mit der Querkraft $Q$, beträgt

$$
v=\frac{z}{2} \cdot \cot \alpha+\frac{b}{2} \cdot \cot \beta
$$

in Richtung des abnehmenden Biegemomentes.

Wird nur ein Teil, $F_{e l}$, der gesamten Längsbiegearmierung $F_{\text {el }}$ ausserhalb des steges in den Flanschen angeordnet und der Rest über dem Steg, so kann das Flanschfachwerk-Modell gemäss Bild 2b durch einen Zugstab in der Stegebene ergänzt werden. In diesem Fall muss sich nur noch derjenige Teil der horizontalen Komponente der Stegdiagonalkraft $D$, welcher dem Verhältnis $\mathrm{F}_{\mathrm{eL}}^{\mathrm{Fl}} / \mathrm{F}_{\mathrm{eL}}$ entspricht, in die beiden Flanschhälften ausbreiten.

Die durch eine Querarmierung aufzunehmende Zugkraft pro Längeneinheit wird

$$
Z=\frac{Q}{2 \cdot Z} \cdot \frac{F_{e L}^{F l}}{F_{e L}} \cdot \tan B \text {. }
$$

Die Armierung kann je zur Hälfte oben und unten in die Platte eingelegt werden (Bild 3 oben). Den beiden Lagen entsprechen die Kräfte

$$
k_{0}=\frac{z}{2} \quad ; \quad k_{u}=\frac{z}{2}
$$




\subsubsection{Längsschub mit Querbiegung}

Für den Fall "Längsschub mit Querbiegung" kann, sofern das unbefriedigende Hauptzugspannungs-Modell ausser acht gelassen wird, die Plattenquerarmierung grundsäzlich nach einem der folgenden drei Modelle bestimmt werden (Bild 3):

\section{a) Ueberlagerung der Armierungen aus zentrisch angreifender Querzugkraft und aus Querbiegung}

Die Armierung für die Querzugkraft $Z$ wird nach dem Flanschfachwerk-Modell gemäss Gl. (4) ermittelt und je zur Hälfte oben und unten in der Platte vorgesehen. Die Armierung für das Querbiegemoment $\bar{M}$ wird gemäss der normalen Biegetheorie bestimmt und der Armierung für Längsschub überlagert. Es braucht also eine obere (Querbiegezugseite) und eine untere (Querbiegedruckseite) Plattenquerarmierung. Diese Armierungen entsprechen den folgenden Kräften:

$$
k_{0}=\frac{z}{2}+\frac{\bar{M}}{z_{p}} \quad ; \quad k_{u}=\frac{z}{2} \text {. }
$$

b) Ueberlagerung der Kräfte aus zentrisch angreifender Querzugkraft und aus Querbiegung

Die zentrisch angreifende Querzugkraft Z nach Flanschfachwerk-Modell gemäss GI. (4) und das Querbiegemoment $\bar{M}$ werden als gemeinsam wirkende Querschnittsbeanspruchung betrachtet. Im Vergleich zum Modell a) bedeutet dies im wesentlichen, dass auf der Querbiegedruckseite die Druckkraft aus dem Querbiegemoment berücksichtigt wird.

$$
k_{0}=\frac{z}{2}+\frac{\bar{M}}{z_{p}} \quad ; \quad k_{u}=\frac{z}{2}-\frac{\bar{M}}{z_{p}} .
$$

Demgemäss wird im Querschnitt meist, d.h. bei genügend grossem Querbiegemoment $\bar{M}$, auf der Querbiegedruckseite keine Querarmierung benötigt.

c) Ueberlagerung der Kräfte aus exzentrisch angreifender Querzugkraft und aus Querbiegung

Die mit der Exzentrizität e gegen die Querbiegedruckzone hin angreifende Querzugkraft $Z$ nach Flanschfachwerk-Model 1 gemäss Gl. (4) und das Querbiegemoment $\bar{M}$ werden als gemeinsam wirkende Querschnittsbeanspruchung betrachtet.

$$
K_{0}=\frac{Z}{2} \cdot\left(1-\frac{2 \cdot e}{z_{p}}\right)+\frac{\bar{M}}{z_{p}} ; \quad K_{u}=\frac{Z}{2} \cdot\left(1+\frac{2 \cdot e}{z_{p}}\right)-\frac{\bar{M}}{z_{p}} \text {. }
$$

Im Vergleich zum Modell b) bedeutet dies eine Verminderung der Beanspruchung auf der Querbiegezugseite, währenddem auf der Querbiegedruckseite, bei genügend grossem Querbiegemoment $\bar{M}$, meist ebenfalls keine Querarmierung benötigt wird.

\subsubsection{Längsschub mit Querbiegung und Quervorspannung}

Für den Fall "Längsschub mit Querbiegung und Quervorspannung" kann ähnlich vorgegangen werden wie beim Fall "Längsschub mit Querbiegung".

Bei den Druckflansch-Untersuchungen ([2] und [4]) wurde bestätigt, dass bei einer plastischen Bemessung die vorgespante Armierung wie eine schlaffe Armierung behandelt werden kann, natürlich unter Berücksichtigung der viel höheren Fliess-bzw. Streckgrenze. Dieses vorgehen ist auch beim Zugflansch anwendbar. 
Um das Verformungs- und Tragverhalten von Zugflanschen von Betonträgern unter Längsschub, Querbiegung und Quervorspannung zu erforschen sowie die im vorangehenden Abschnitt beschriebenen Modelle zu überprüfen, wurde ein Versuchsprogramm ausgearbeitet. Dieses sah die Prüfung von sechs Versuchsträgern, Z1 bis Z6, vor. Die ersten zwei Träger, Z1 und Zz, wurden dem Problemkreis "Längsschub allein" zugeordnet. Bei den anderen vier Trägern, Z3 bis Z6, wurde je die eine Trägerhälfte dem Problemkreis "Längsschub mit Querbiegung" und die andere Trägerhälfte dem Problemkreis "Längsschub mit Querbiegung und Quervorspannung" gewidmet.

Die Belastungsanordnung der sechs Versuchsträger ist in Bild 10 dargestellt. Bei allen Trägern waren die Abmessungen, die Betonqualität und die Stegarmierung gleich gehalten; sie sind in Bild 5 ersichtlich.

Die Längsbiegearmierung der Träger wurde je zur Hälfte in beiden Flanschhälften angeordnet, mit Ausnahme des Trägers 22 . Bei diesem Träger wurden nur $75 \%$ der Längsbiegearmierung im Flansch und $25 \%$ über dem steg eingelegt.

Für die Planung und Bemessung der Versuchsträger wurde im Flanschfachwerk-Modell mit der Querzugkraft gemäss GI. (4) der Ausbreitwinkel $\beta$ zu $33^{\circ}$ angenommen, was

$\tan \beta=0.66$

entspricht.

Für das Versatzmass $v$ der Querarmierung, bezogen auf den Querschnitt mit der Querkraft Q, gemäss G1. (3), wurde für $\alpha$, entsprechend den steilen Druckdiagonalen beim Zwischenauflager, $\simeq 60^{\circ}$ und $f u ̈ r ~ \beta \simeq 30^{\circ}$ eingesetzt. Damit ergibt sich gerundet

$$
v \simeq 0.3 \cdot\left(z+2 \cdot b_{e}\right)
$$

Die Plattenquerarmierungen wurden nach den in Bild 3 dargestellten Modellen bemessen. Bild 4 zeigt die entsprechenden Fliesskräfte der eingelegten Querarmierungen. Dort, wo nach Modell keine oder nur eine schwache Querarmierung erforderlich gewesen wäre, wurde stets eine konstruktive Mindestarmierung eingelegt.

Im einzelnen wurde die rechnerisch ermittelte Plattenquerarmierung wie folgt konzipiert:

\section{Träger 21 (Längsschub allein)}

Die Plattenquerarmierung wurde nach dem Flanschfachwerk-Modell gemäss den Gleichungen (4) und ( 9 ) bestimmt und im allgemeinen um das Versatzmass $v$ gemäss G1. (10) in Richtung des abnehmenden Biegemomentes verschoben je zur Hälfte oben und unten in die Platte eingelegt.

Auf der linken Seite des Trägers wurde jedoch das Versatzmass $v$ am Trägerende etwa um $30 \%$ (ca. $12.5 \mathrm{~cm}$ ) verkürzt und die Plattenquerarmierung am Ende entsprechend zusammengeschoben. Das Gesamtvolumen der Plattenquerarmierung wurde somit, im Vergleich zur rechten Seite des Trägers, nicht verändert.

\section{Träger Z2 (Längsschub allein)}

Die Plattenquerarmierung wurde auch bei diesem Träger grundsätzlich nach dem Flanschfachwerk-Modell gemäss den Gleichungen (4), (9) und (10) bestimmt. Weil jedoch nur $75 \%$ der Längsbiegearmierung im Flansch und $25 \%$ über dem Steg angeordnet wurden, betrug die er- 
forderliche Fliesskraft der Querarmierung nur 75\% derjenigen des Trägers Z1. Diese Armierung wurde auf der rechten Seite des Trägers eingelegt. Auf der linken Seite wurden $30 \%$ mehr Querarmierung, d.h. wie bei 21 , angeordnet.

\section{Träger 23 (Längsschub mit Querbiegung/ und Quervorspannung)}

Die Plattenquerarmierung dieses Trägers wurde nach dem Modell "Ueberlagerung der Armierungen aus zentrisch angreifender Querzugkraft und aus Querbiegung" bemessen. Die Querzugkraft $Z$ wurde nach dem Flanschfachwerk-Modell gemäss den Gleichungen (4), (9) und (10) berechnet.

Auf der rechten Seite des Trägers wurde die Zugplatte in Querrichtung teilweise vorgespannt, d.h. es wurde auf der oberen Seite (Querbiegezugseite) eine gemischte (schlaffe und vorgespannte) Querarmierung angeordnet.

\section{Träger 24 (Längsschub allein, mit Querbiegung/ und Quervorspannung)}

Die Plattenarmierung dieses Trägers wurde nach dem Modell "Ueberlagerung der Kräfte aus exzentrisch angreifender Querzugkraft und aus Querbiegung" bemessen. Die Querzugkraft $Z$ wurde nach dem Flanschfachwerk-Modell gemäss den Gleichungen (4), (9) und (10) berechnet. Für die Exzentrizität e (zur Querbiege-Druckzone hin) wurde der "Extremwert" $z_{p} / 2$ gewählt. Als Unterschied zu den Trägern Z3, Z5 und Z6 wurde die Querbiegung um ca. 50\% erhöht, wodurch die unten (auf der Querbiegedruckseite) angreifende Druckkraft aus der Querbiegung $\bar{M} / z_{p}$ gleich gross war wie die (als dort angreifend angenommene) Querzugkraft $z$, d.h. die Kraft $K_{u}$ gemäss 61 . (8) war null. Daher wurde auf der Querbiegedruckseite nur die Mindestarmierung eingelegt. Von dieser Mindestarmierung abgesehen, war die Zugplatte des Trägers 24 sozusagen nur auf Querbiegung armiert (obere Lage).

\section{Träger 25 (Längsschub mit Querbiegung/ und Quervorspannung)}

Die Plattenquerarmierung dieses Trägers wurde nach dem Modell "Ueberlagerung der Kräfte aus zentrisch angreifender Querzugkraft und aus Querbiegung" bemessen. Die Querzugkraft Z wurde auch hier nach dem Flanschfachwerk-Modell gemäss den Gleichungen (4), (9) und (10) berechnet. Als Unterschied zum Träger 23 wurde gemäss Gl. (7) unten (auf der Querbiegedruckseite) die Biegedruckkraft aus der Querbiegung $\bar{M} / z_{p}$ in Abzug gebracht. Hier war die Biegedruckkraft ca. $33 \%$ grösser als der untere Querzugkraft-Anteil $2 / 2$.

Auch bei diesem Träger wurde die Platte auf der rechten Seite teilweise vorgespannt.

\section{Träger Z6 (Längsschub mit Querbiegung/ und Quervorspannung)}

Die Plattenquerarmierung dieses Trägers wurde nach dem Modell "Ueberlagerung der Kräfte aus exzentrisch angreifender Querzugkraft und aus Querbiegung" bemessen. Die Querzugkraft $Z$ wurde wiederum nach dem Flanschfachwerk-Modell gemäss den Gleichungen (4), (9) und (10) berechnet. Für die Exzentrizität e (gegen die Querbiegedruckzone hin) wurde der "Mittelwert" $z_{p} / 4$ gewählt. Die unten (auf der Querbiegedruckseite) angreifende Druckkraft aus der Querbiegung $\bar{M} / z_{p}$ betrug nur ca. $89 \%$ des unteren Querzugkraft-Anteils $3 \cdot z / 4$. Die somit noch resultierende, verhältnismässig kleine Zugkraft $K_{u}$ gemäss Gl. (B) war durch die Mindestarmierung reichlich abgedeckt.

Auch bei diesem Träger wurde die Platte auf der rechten Seite teilweise vorgespannt.

Die Quervorspannung der Zugflanschen auf der rechten Seite der Träger Z3 bis Z6 wurde konstant längs der Trägerhälften aufgebracht. Ihre Grösse wurde so gewählt, dass in den Bereichen III "Reine Querbiegung" (Bild 4b) das Fliesskraftverhältnis

$$
\lambda=\frac{F_{s} \cdot \sigma_{s f}}{F_{e} \cdot \sigma_{e f}+F_{s} \cdot \sigma_{s f}}
$$


0.6 betrug. Dies entspricht einer häufig anzutreffenden Quervorspannung von Brückenfahrbahnplatten.

Für das Verhalten von teilweise vorgespannten Konstruktionen im Gebrauchszustand ist der Vorspanngrad

$$
k^{\prime}=\frac{M_{z u I, V}}{M_{g+p}}
$$

von wesentlicher Bedeutung. Der Vorspanngrad $K^{\prime}$ gibt an, bei welchem Teil des Gebrauchslastmomentes am ungerissenen Querschnitt die Randzugspannung $\sigma_{r}$ auftritt. Er wird in Tabelle 7 für reine Biegung $(\gamma=0)$ und für Biegung mit zentrischem Zug $(\gamma>0)$ näher definiert.

Vor allem die Randzugspannung $\sigma_{r}$, aber auch das Zugkraft-Momenten-Verhältnis

$$
\gamma=\frac{Z}{M / d}
$$

haben einen wesentlichen Einfluss auf den Vorspanngrad. Dies zeigt auch Tabelle 7 , wo die Zusammenhänge für den Anschnitt Zugplatte - Steg der Versuchsträger Z3, Z5 und Z6 dargestellt sind. Nach der Norm SIA 162 [5] beträgt die zulässige Randspannung $\sigma_{r}$ bei voller Vorspannung 10 (bis 20 ) $\mathrm{kg} / \mathrm{cm}^{2}$.

Bei den Versuchsträgern 23, 25 und Z6 betrug der Vorspanngrad bei der Bemessungsfliesslast für $\sigma_{r}=10 \mathrm{~kg} / \mathrm{cm}^{2}$ in den Bereichen III "Reine Querbiegung" $(\gamma=0) 0.68$ und im Bereich IV "Längsschub mit Querbiegung" ( $\gamma \cong 1.8) 0.52$. 


\section{VERSUCHSTRAEGER}

2. 1 Beschreibung

\subsubsection{Abmessungen, Armierungen}

Die Abmessungen und Armierungen gehen aus den Bildern 5 und 6 hervor.

\section{Abmessungen}

Die Betonabmessungen waren bei allen sechs Trägern gleich. Die Träger waren $616 \mathrm{~cm}$ lang und $63 \mathrm{~cm}$ hoch, der Steg $20 \mathrm{~cm}$ breit und die Flanschen $100 \mathrm{~cm}$ breit. Die Dicke der Druckplatte betrug am Rand $10 \mathrm{~cm}$ und beim Steg $15 \mathrm{~cm}$, diejenige der Zugplatte $10 \mathrm{~cm}$.

\section{Längsbiegearmierung}

Die Längsbiegearmierung war mit Ausnahme von 22 bei allen sechs Trägern gleich. Sie bestand aus der hauptarmierung, 8 stäbe $\oslash 26 \mathrm{~mm}$, und aus den konstruktiv erforderlichen Stäben $2 \emptyset 8 \mathrm{~mm}$ und 4 $06 \mathrm{~mm}$. Beim Träger 22 wurden 2 der 8 Stäbe $026 \mathrm{~mm}$ über dem Steg angeordnet anstelle der 2 Stäbe $\emptyset 8 \mathrm{~mm}$. Sämtliche Stäbe der Hauptarmierung wurden mit stumpf angeschweissten Endplatten verankert.

\section{Schubarmierung im Steg}

Die Schubarmierung im Steg war bei allen sechs Trägern gleich. Sie bestand in den Querkraftbereichen aus Bügeln $\emptyset 12 \mathrm{~mm}$ im Abstand $t=12.5 \mathrm{~cm}$ und ausserhalb derselben aus Bügeln $\oslash 8 \mathrm{~mm}$ im Abstand $t=25 \mathrm{~cm}$.

\section{Querarmierung in der Druckplatte}

Die nach den entsprechenden Modellen unter Berücksichtigung des Versatzmasses (siehe [1] und [3]) bemessene Querarmierung in der Druckplatte bestand in den Längsschubbereichen und ausserhalb derselben aus Bügeln $\emptyset 6 \mathrm{~mm}$ im Abstand $t=25 \mathrm{~cm}$.

\section{Querarmierung in der Zugplatte}

Die schlaffe Querarmierung in der Zugplatte bestand aus Armierungsstäben 0 mm und Ø $8 \mathrm{~mm}$, die in zwei Lagen, d.h. oben und unten, eingelegt wurden. Die genaue Anordnung der Plattenarmierung ist aus Bild 6 ersichtlich.

Als vorgespannte Plattenquerarmierung wurden bei den Trägern Z3, Z5 und Z6 Spanndrähte $\emptyset 4 \mathrm{~mm} \mathrm{im}$ Abstand $t=20 \mathrm{~cm}$ und beim Träger $Z 4$ Spanndrähte $\emptyset 4 \mathrm{~mm}$ im Abstand $t=13.3 \mathrm{~cm}$ eingelegt. Um einen einwandfreien Verbund sicherzustellen und nicht ein kleines Hüllrohr injizieren zu müssen, wurde folgende Lösung gewählt (Bild 7): Beim Betonieren wurden in der Zugplatte kanalförmige, d.h. oben offene, Aussparungen angebracht. In diese konnten die Spanndrähte eingelegt werden. Nach dem Vorspannen wurden dịe Aussparungen mit erdfeuchtem Zementmörtel ausgefüllt, bzw. ausgestopft.

Die Vorspannkräfte, entsprechend einer Spannung $\sigma_{s v}(t=0)=0.7 \beta_{z d}$, wurden mit einem Dynamometer gemessen. Der Spannungsabfall infolge von Schwinden, Kriechen und Relaxation wurde bis zum Prüftag zu $10 \%$ (wie in [2] und [4]) angenommen.

\subsubsection{Herstellung, Vorspannung, Lagerung}

Die Herstellung der sechs Versuchsträger, Z1 bis Z6, erfolgte in einer Holzschalung. Der Beton wurde mit einem Nadelvibrator verdichtet. Fünf Tage nach dem Betonieren wurden die Träger ausgeschalt. Im Alter von 28 Tagen wurden die Vorspanndrähte angebracht und vorgespannt. Anschliessend erfolgte das oben beschriebene Injizieren. Bis zur Prüfung im Alter von 56 Tagen wurden die Träger im Betonierraum gelagert, wo sie für die Prüfung vorbereitet wurden. 


\subsubsection{Armierungsstah1, Spannstahl}

Die gesamte schlaffe Armierung bestand aus naturhartem Stahl (BOX-ULTRA) mit Ausnahme der Querarmierungsstäbe $\emptyset 6 \mathrm{~mm}$ im Zugflansch. Diese Armierungsstäbe bestanden aus kaltverformtem Stahl (TOR), der aber durch eine spezielle Wärmebehandlung im Werk wieder eine ausgeprägte Fliessgrenze erhalten hatte.

Die vorgespannte Querarmierung der Zugplatte bestand aus patentiert kaltgezogenen Spanndrähten $\emptyset 4 \mathrm{~mm}$ (Stahlton $A G$ ).

Die Kennwerte (Mittelwerte) der verwendeten Stähle sind in Tabelle 1 zusammengestellt. Sie wurden in einer dehnungsgesteuerten Zugmaschine ermittelt (wie bei [1] und [2]). Dabei wurde die Dehnungsgeschwindigkeit auf $1.25 \%$ pro Minute festgelegt. Im Fliessbereich und bei der Höchstlast wurde die Dehnungsgeschwindigkeit gleich null gesetzt. Während einer Wartezeit von zwei Minuten fiel der sogenannte dynamische Festigkeitswert ( $\sigma_{f d}$ $\left.b z w . \beta_{z d}\right)$ auf den statischen Wert $\left(\sigma_{f s} b z w . \beta_{z s}\right)$ ab. Wartezeiten länger als zwei Minuten haben praktisch keinen Einfluss auf die statischen Festigkeitswerte.

Die gemittelten idealisierten $\sigma-\varepsilon$-Diagramme der Querarmierung für die Zugplatte sind in Bild 8 dargestellt.

\section{$\underline{2.2 .2 \text { Beton }}$}

Die Zusammensetzung des Frischbetons war für alle sechs Träger gleich und ist in Tabelle 2 angegeben. Der Zuschlagstoff, der aus gut gewaschenem und getrocknetem Material bestand, wurde getrennt nach drei Komponenten beigegeben. Die Siebkurve der Mischung entsprach ungefähr der Fuller-Kurve. Als Bindemittel wurde normaler Portlandzement verwendet.

Zur Bestimmung der konventionellen Betonfestigkeitswerte Würfeldruckfestigkeit $\beta_{W}$ ' Prismendruckfestigkeit $\beta_{p}$, Biegezugfestigkeit $\beta_{b z}$ und Querzugfestigkeit $\beta_{q z}$ wurden Würfel $(15 \times 15 \times 15 \mathrm{~cm})$, Prismen $(12 \times 12 \times 36 \mathrm{~cm})$ und zylinder $(\emptyset 10 \mathrm{~cm}, \mathrm{~h}=30 \mathrm{~cm}$ ) hergestellt und geprüft. Die Mittelwerte dieser im Alter von 28 Tagen geprüften Proben sind in Tabelle 3 aufgeführt.

Für die Ermittlung des $\sigma-\varepsilon-D i a g r a m m s$ des Betons wurden Prüfzylinder verwendet. Um den Einfluss der Dehnungsgeschwindigkeit demjenigen der Balkenversuche anzupassen, wurde eine verhältnismässig geringe Dehnungsgeschwindigkeit van etwa $0.07 \%$ pro Minute gewählt. Das Ergebnis ist in Tabelle 4 und in Bild 9 dargestellt.

\subsubsection{Zementmörtel}

Die Zusammensetzung des für die "Injektion" der Spanndrähte verwendeten frischen Zementmörtels ist in Tabelle 5 angegeben.

Zur Ermittlung der konventionellen Mörtelfestigkeitswerte $\beta_{p}$ und $\beta_{b z}$ wurden kleine Prismen $(4 \times 4 \times 16 \mathrm{~cm})$ hergestellt und geprüft. Die Mittelwerte dieser im Alter von 28 Tagen geprüten Proben sind aus Tabelle 6 ersichtich. 


\subsubsection{Berechnete Lasten und Schubspannungen}

Der Bemessung der Träger wurden die mit den effektiven Materialkennwerten berechneten theoretischen Last- und Schnittkraftgrössen zugrunde gelegt.

In Tabelle $B$ sind die Längsbelastung $P_{u B}^{T h}$ für die theoretische Biegekapazität der Träger (Biegefliesslast) sowie die Längs - und Querbelastung $P_{f}^{T h}$ und $\bar{P}_{f}^{T h}$ für den theoretischen Fliessbeginn der Zugplattenquerarmierung (Bemessungsfliesslasten) aufgeführt. Die Bemessungsfliesslast $P_{f}^{T h}$ wurde, mit Ausnahme der linken Seite von 22 , auf etwa $70 \%$ der Biegefliesslast $P_{\text {UB }}^{\text {Th }}$ festgelegt.

Ebenfalls in Tabelle 8 aufgeführt sind die nominellen Schubspannungen $\tau_{s}$ ' $\tau_{y x} u n d \tau_{y z}$, die sich unter diesen Lasten im Steg bzw. im Anschnitt Zugplatte - Steg ergeben. Sie wurden wie folgt definiert:

- Nominelle Schubspannung im Steg:

$$
\tau_{s}=\frac{Q}{b \cdot z}=\frac{P^{T h}}{b \cdot z}
$$

wobei: $\quad z=$ innerer Hebelarm des Trägers $(50 \mathrm{~cm})$

- Nominelle Längsschubspannung im Anschnitt Zugplatte - Steg:

$$
\tau_{y x}=\frac{Q}{2 \cdot d \cdot z} \cdot \frac{F_{e L}^{F l}}{F_{e L}}=\frac{P_{f}^{T h}}{2 \cdot d \cdot z} \cdot \frac{F_{e L}^{F l}}{F_{e L}}
$$

wobei: $\quad F_{\text {eL }}^{F l}=$ Längsbiegearmierung im Flansch

$F_{e L}=$ gesamte Längsbiegearmierung

- Nominelle Querschubspannung im Anschnitt Zugplatte - Steg:

$$
\tau_{y z}=\frac{\bar{P}_{f}^{T h}}{I \cdot z_{p}}
$$

$$
\text { wobei: } \begin{aligned}
& 1 \\
& =\text { Abstand der Querbelastung } \bar{P}(88 \mathrm{~cm}) \\
z_{p} & =\text { innerer Hebelarm der Zugplatte }(8.2 \mathrm{~cm})
\end{aligned}
$$

Eine Längsbelastung $P_{f}^{T h}$ von 43 t erzeugt im Anschnitt Zugplatte - Steg eine nominelle Längsschubspannung $\tau_{y x}$ von $43 \mathrm{~kg} / \mathrm{cm}^{2} \mathrm{bzw}$. $32 \mathrm{~kg} / \mathrm{cm}^{2}$ beim Träger $Z 2$. Die bei den vorliegenden Versuchen nicht weiter berücksichtigte Querschubspannung $\tau$ yz infolge einer Querbelastung $\bar{P}_{f}^{T h}$ von $3.9 \mathrm{t}$, bzw. $6.0 \mathrm{t}$ beim Träger 24 , betrug $5.4 \mathrm{~kg} / \mathrm{cm}^{2} \mathrm{bzw} .8 .3 \mathrm{~kg} / \mathrm{cm}^{2}$.

\subsubsection{Längsbiegearmierung}

Die Längsbiegearmierung wurde für die angestrebte Biegefliesslast P Th nach den Regeln der plastischen Bemessung bestimmt. Dabei wurde für den Stahl der Mittelwert der dynamischen Fliessspannung $\sigma_{f d}=5.2 \mathrm{t} / \mathrm{cm}^{2}$ und für den Beton der Mittelwert der konventionell ermittelten Würfeldruckfestigkeit $B_{w 28}=375 \mathrm{~kg} / \mathrm{cm}^{2}$ eingesetzt. Der Längsbiegearmierungsgehalt $\mu_{L}$ betrug $0.78 \%$. 


\subsubsection{Schubarmierung im Steg}

Die Schubarmierung im Steg wurde ebenfalls auf die Biegefliesslast $P_{u B}^{T h}$ ausgelegt. Um das Risiko eines vorzeitigen Schubbruchs zu eliminieren, wurde die Bemessung der Bügel nach der Richtlinie 34 der Norm SIA 162 mit tan $\cong 0.7$ durchgeführt. In den Bereichen ohne Querkraft wurde eine minimale Bügelarmierung eingelegt. Der Schubarmierungsgehalt im Steg $\mu_{\theta}$ betrug $0.9 \%$ bzw. $0.2 \%$ in den Bereichen ohne Querkraft.

\subsubsection{Querarmierung in der Druckplatte}

Die Querarmierung in der Druckplatte wurde ebenfalls auf die Biegefliesslast $P_{u B}^{T h}$ ausgelegt. Die Bemessung erfolgte nach dem entsprechenden Flanschfachwerk-Modell für den Druckflansch (siehe [1] und [3]).

Def Querarmierungsgehalt in der Druckplatte (obere und untere Armierung zusammen) betrug $0.3 \%$ bzw. $0.15 \%$ in den Bereichen, für welche das Modell keine Armierung vorschreibt.

\subsubsection{Querarmierung in der Zugplatte}

\section{Träger Z1 und Z2 (Längsschub allein)}

Die Queràmierung in den Zugplatten der Träger $Z 1$ und $Z 2$ wurde auf die Bemessungsfliesslast $P_{f}^{T h}=43 t$ ausgelegt, mit Ausnahme der linken Trägerhälfte des Trägers 22 , für welche entsprechend der um $30 \%$ vergrösserten Querarmierung (siehe Abschnitt 1.4 und Bild 4a) eine Bemessungsfliesslast van $56 \mathrm{t}$ angesetzt wurde.

Die Zugplatten dieser beiden Versuchsträger können aufgrund des Flanschfachwerk-Modells in zwei verschieden beanspruchte Bereiche aufgeteilt werden (Bild $4 a$ ):

- Bereich I : "Keine Querbeanspruchung"

- Bereich II: "Längsschub allein"

Im Bereich I "Keine Querbeanspruchung" wurden die Zugplatten beider Träger mit einer zweilagigen konstruktiven Mindestarmierung versehen ( $\mu_{\min }=0.12 \%$ pro Lage).

Im Bereich II "Längsschub allein" wurde die Plattenquerarmierung nach dem FlanschfachwerkModell gemäss den Gleichungen (4) und ( 9 ) bemessen und je zur Hälfte oben und unten in die Zugplatte eingelegt. Der Armierungsgehalt pro Lage betrug beim Träger $Z 1 \mu=0.32 \%$ und beim Träger $22 \mu=0.25 \%$ in der rechten Trägerhälfte, bzw. ebenfalls $\mu=0.32 \%$ in der linken Trägerhälfte.

\section{Träger 23 bis Z6 (Längsschub mit Querbiegung/ und Quervorspannung)}

Der Bemessung der Querarmierung in der Zugplatte der Träger Z3, Z5 und Z6 wurden die Fliesslasten $P_{f}^{T h}=43 \mathrm{t}$ und $\bar{P}_{f}^{T h}=3.9 \mathrm{t}$ zugrunde gelegt. Beim Träger $Z 4$ wurde die Bemessungsfliesslast $\bar{P}_{f}^{\top h}$ auf $6.0 \mathrm{t}\left(\bar{M} / z_{p} \cong Z\right)$ angesetzt.

Die Zugplatten dieser vier Versuchsträger können ebenfalls in zwei verschieden beanspruchte Bereiche aufgeteilt werden (Bild 4b):

- Bereich III: "Reine Querbiegung"

- Bereich IV : "Längsschub mit Querbiegung"

Bei allen vier Versuchsträgern wurden zudem die Zugplatten in der rechten Trägerhälfte in Querrichtung teilweise vorgespant.

Im_Bereich_III "Reine Querbiegung". wurde die Querarmierung allein auf die Bemessungsfliesslast $\bar{P}_{f}=3.9 \mathrm{t}$, bzw. $6.0 \mathrm{t}$ beim Träger 24 ausgelegt. Die im Abstand von $44 \mathrm{~cm}$ längs den Plattenrändern wirkende Querbelastung $\bar{P}_{f}^{T h} / 2$ (Bild 10) ergab im Anschnitt Zugplatte - Steg ein Querbiegemoment $\bar{M}$ von $1.46 \mathrm{mt} / \mathrm{m}^{\prime}$, bzw. $2.24 \mathrm{mt} / \mathrm{m}^{\prime}$ beim Träger $Z 4$. 
Die Armierungen wurden nach den üblichen Regeln der plastischen Bemessung bestimmt und oben (auf der Querbiegezugseite) in der Platte eingelegt. Der Anteil der vorgespannten Querarmierung (auf der rechten Trägerseite) kann durch das Fliesskraft-Verhältnis $\lambda$ beschrieben werden (siehe Abschnitt 1.4). Es betrug bei allen vier Versuchsträgern im Bereich III etwa 0.6. Die entsprechenden Vorspanngrade $\kappa^{\prime}$ sind für die Träger 23,25 und Z6 in Tabelle 7 eingetragen (reine Biegung, $\gamma=0$ ).

Der absolute Armierungsgehalt der oberen Querarmierung $\mu=\left(F_{e}+F_{s}\right) / b \cdot h_{p}$ betrug $0.38 \%$ in den schlaff armierten Trägerhälften und $0.22 \%$ in den teilweise vorgespannten Trägerhälften, bzw. $0.59 \%$ und $0.34 \%$ beim Träger 24 . In der unteren Lage wurde auch hier eine konstruktive Mindestarmierung $\left(\mu_{\min }=0.12 \%\right)$ angeordnet.

Im_Bereich IV_"Längsschub mit Qquerbiegung". wurde die Querarmierung auf die Bemessungsfliesslasten $P_{f}^{T h}=43 t$ und $\bar{P}_{f}^{T h}=3.9 \mathrm{t}$, bzw. $6.0 \mathrm{t}$ beim Träger $Z 4$, ausgelegt. Dabei wurde von den Beanspruchungen im Anschnitt Zugplatte - Steg gemäss den in Abschnitt 1.3 dargestellten Modellen ausgegangen.

In den teilweise vorgespennten Zugplatten (rechte Trägerhälften) wurde die im Bereich III mit dem Fliesskraftverhältnis $\lambda=0.6$ bestimmte und oben eingelegte vorspannarmierung konstant auch über den Bereich IV durchgeführt und mit der erforderlichen schlaffen Armierung ergänzt. Die entsprechenden Vorspanngrade $K^{\prime}$ der Versuchsträger 23,25 und 26 bei den Bemessungsfliesslasten $P_{f}^{T h}, \bar{P}_{f}^{T h}$ sind in der Tabelle 7 eingetragen $(\gamma=1.8)$.

Der absolute Armierungsgehalt der oberen Querarmierung $\mu=\left(F_{e}+F_{s}\right) / b \cdot h_{p}$ schwankte in den nur schlaff armierten Trägerhälften zwischen $0.53 \%$ beim Träger 26 und $0.69 \%$ bei den Trägern 23 und 25 . In den teilweise vorgespannten Trägerhälften betrug er $0.37 \%$ beim Träger $Z 6$ und $0.51 \%$ bei den Trägern $Z 3$ und $Z 5$. In der unteren Lage, wo gemäss Modell keine Armierung vorgesehen ist, wurde eine konstruktive Mindestarmierung $\left(\mu_{\min }=0.12 \%\right)$ eingelegt. 


\subsection{Versuchsanlage}

Die Versuchsträger $Z 1$ bis $Z 6$ wurden auf dem neuen Aufspannboden der Eidgenössischen Technischen Hochschule Zürich auf dem Hönggerberg geprüft. Bild 11 zeigt die Versuchsanlage für die Träger $Z 1$ und $Z 2$ und Bild 12 diejenige für die Träger $Z 3$ bis $Z 6$. Es wurden insgesamt zwei Arten von Belastungen aufgebracht (vgl. Bild 10):

- Die "Längsbelastung" P wurde durch zwei 100 t Pressen erzeugt. Um während des Versuchs eine symmetrische und zwängungsfreie Verschiebung der beiden Trägerauflager zu gewährleisten, wurden die dortigen blockierbaren Rollenlager abwechslungsweise gelöst bzw. blockiert.

- Die "Querbelastung" $\bar{P}$ wurde durch sieben kleine Pressen ( $15 \mathrm{t}$ ) im Abstand $1=88 \mathrm{~cm}$ aufgebracht, welche an eine zweite unabhängige Pumpanlage angeschlossen waren. Jeder Kolben zieht die Zugplatte mit der Kraft $2 \times \bar{P}$ nach unten, wobei die Reaktion dieser Kraft von unten auf den Steg des Trägers abgegeben wird. Die Querbelastung $\bar{P}$ bildet zusammen mit den entsprechenden Reaktionen einen Eigenspannungszustand und verursacht somit in Längsrichtung des Trägers keine zusätzlichen Beanspruchungen und somit auch keine zusätzlichen Längsschubspannungen $\tau_{y x}$. Abgesehen von den versuchstechnischen Vorteilen dieser Belastungsanordnung wird damit auch ein direkter Vergleich zwischen aufgebrachter Belastung $P$ bzw. $\bar{P}$ und Beanspruchung in der Zugplatte ermöglicht. Um beim Anschnitt Zugplatte - Steg längs des Trägers ein möglichst gleichmässiges Querbiegemoment $\bar{M}$ zu erzeugen, wurden die Angriffspunkte der Zugkräfte $\bar{P}$ am Zugplattenrand durch kleine Lastverteilträger (HEB 100) verdoppelt.

\subsection{Versuchsablauf}

Bei jeder Laststufe kam das übliche Belastungsschema zur Anwendung, nämlich:

- Verändern der Last innerhalb von etwa zwei Minuten bis zum Erreichen der vorgesehenen Last- bzw. Verformungsgrösse. Ablesen der Anfangslast $P_{A}$ bzw. $\bar{P}_{A}$.

- Konstanthalten der Anfangslast $P_{A}$ bzw. $\bar{P}_{A}$ während zwei Minuten.

- Konstanthalten einer Deformationsgrösse für die Dauer der Messungen (ca. 45 Minuten); entsprechende Reduktion der Last (Relaxation). Nach Beendigung der Messungen Ablesen der Endlast $P_{E}$ bzw. $\bar{P}_{E}$.

In der dritten Phase wurden als Deformationsgrössen für die Längsbelastung $P$ jeweils die Durchbiegung unter der Lasteintragung und für die Querbelastung $\bar{P}$ die relative Durchbiegung des Zugplattenrandes gegenüber dem Steg in Trägermïte (Bereich III: "Reine Querbiegung") konstant gehalten.

Es wurden folgende charakteristische Lastgrössen definiert:

$$
\begin{array}{lll}
P_{I}=P_{f}^{T h} / 1.8=24 t, & \bar{P}_{I}=\bar{P}_{f}^{T h} / 1.8=2.15 t \quad(3.3 \mathrm{t} \text { bei Z4) } \\
P_{I I}=P_{f}^{T h} / 1.3=33 \mathrm{t}, & \bar{P}_{I I}=\bar{P}_{f}^{T h} / 1.3=3.00 t(4.6 \mathrm{t} \text { bei z4) } \\
P_{I I I}=P_{f}^{T h}=43 \mathrm{t}, & \bar{P}_{I I I}=\bar{P}_{f}^{T h}=3.90 \mathrm{t}(6.0 \mathrm{t} \text { bei Z4) }
\end{array}
$$

Der Belastungsablauf der Versuchsträger ist in den Bildern 13, 14 und 15 aufgezeichnet. Die Lasten der einzelnen Laststufen sind aus den Tabellen 9 bis 14 ersichtlich. 
Die Träger $Z 1$ und $Z 2$ wurden nur einer Längsbelastung $P$ unterworfen (Bild 13).

Beim Träger 24 (Querarmierung nur in der oberen Lage - abgesehen von der Mindestarmierung in der unteren Lage) wurde zuerst mit der Längsbelastung $P$ bis auf $P_{\text {III }}$ gefahren (Bild 14). Von da an wurde die Längsbelastung $P$ konstant gehalten bzw. nach jeder Laststufe wieder auf die Anfangslast $P_{A}=P_{I I I}$ erhöht und die Querbelastung $\bar{P}$ stufenweise $\left(\bar{P}_{I}, \bar{P}_{I I}\right.$ und $\left.\bar{P}_{I I I}\right)$ bis zum Bruch vergrössert.

Bei den Trägern Z3, Z5 und Z6 hingegen (Bild 15) wurde zuerst die Querbelastung $\bar{P}$ auf eine charakteristische Lastgrösse ( $\bar{P}_{I}$ bzw. $\bar{P}_{I I}$ bzw. $\bar{P}_{\text {III }}$ ) vorgezogen. Anschliessend wurde mit der Längsbelastung $P$ auf die entsprechende Lastgrässe ( $P_{I}$ bzw. $P_{\text {II }}$ bzw. $P_{\text {III }}$ ) nachgefahren. Von $P_{I I I}, \bar{P}_{I I I}$ an wurde die Querbelastung $\bar{P}$ konstant gehalten bzw. nach jeder Laststufe wieder auf die Anfangslast $\bar{P}_{A}=\bar{P}_{I I I}$ erhöht und nur die Längsbelastung $P$ bis zum Bruch weiter vergrössert.

\subsection{Messungen}

\subsubsection{Lasten}

Die in den Versuchsträgern wirksamen Schnittkräfte wurden aus den gemessenen Lasten $P$ und $\bar{P}$ ermittelt. Die Längsbelastung $P$ wurde durch zwei Druckmessdosen direkt bei der Krafteinleitung unter den Pressen erfasst. Die Querbelastung $\bar{P}$ konnte am geeichten Pendelmanometer abgelesen werden.

\subsubsection{Verformungen}

Es wurden folgende Verformungsmessungen durchgeführt:

\section{Durchbiegungen}

Die Durchbiegungen der Versuchsträger (Bild 16) wurden mittels fest installierter induktiver Weggeber zwischen Hallenboden und Unterkante Versuchsträger gemessen (Anzeige in $5 / 1000 \mathrm{~mm}$ ).

\section{Stauchungen der Druckplatte}

Mit einem elektronischen Deformeter van $20 \mathrm{~cm}$ Basislänge (Anzeige in $1 / 1000 \mathrm{~mm}$ ) wurden auf der unteren Seite der Druckplatte die Betonstauchungen in Längsrichtung entlang des Trägers gemessen (Bild 16).

\section{Dehnungen der Längsbiegearmierung}

Mit einem elektronischen Deformeter von $20 \mathrm{~cm}$ Basislänge (Anzeige in 1/1000 mm) wurden jeweils die Dehnungen eines Stabes der Längsbiegearmierung in der oberen Lage entlang der ganzen Zugplatte gemessen (Bild 17). Beim Versuchsträger Z2 wurden die Dehnungen zweier Armierungsstäbe (einer im Flansch und einer über dem Steg) entlang einer Trägerhälfte gemessen (Bild 18).

\section{Dehnungen der Querarmierung in der Zugplatte}

Mit einem elektronischen Deformeter von $10 \mathrm{~cm}$ Basislänge (Anzeige in 1/1000 mm) wurden die Dehnungen der schlaffen oberen Plattenquerarmierung an fünf Messstrecken pro Armierungsstab gemessen. An der unteren Plattenquerarmierung wurden die Dehnungen an zwei Messstrecken pro Armierungsstab gemessen (siehe z.B. Bild 19b oben).

Die Deformeter wurden auf Messbolzen gesetzt, die mit einem Schneliklebestoff auf den freiliegenden StahI bzw. auf die Betonfläche geklebt waren. Mit diesem Verfahren konnten die Messungen bis zum Bruch einwandfrei vorgenommen werden. 
Bei der Auswertung der Dehnungsmessungen der Plattenquerarmierung wurde ferner der Einfluss der Plattenkrümmung infolge von Querbiegung auf die Messwerte "auskorrigiert" (Bild 46). Die an der oberen bzw. unteren Plattenquerarmierung ermittelten Dehnungen $\varepsilon_{0}$ und $\varepsilon_{u}$ wurden nicht in der Mittellinie der Armierungsstäbe gemessen, sondern auf der Höhe des Messbolzens. Die effektiven Stabdehnungen $\varepsilon_{\text {eo }}$ bzw. $\varepsilon_{\text {eu }}$ wurden anhand der "gemessenen" Dehriungsebene $\left(\varepsilon_{0}, \varepsilon_{u}\right)$ bestimmt.

Ferner wurde für die Umrechnung der Dehnungen auf Spannungen folgender Effekt berücksichtigt: Mit einem Deformeter wird eine Längenänderung $\Delta l$ der Basislänge $l_{0}$ gemessen. Bildet man den Quotienten $\Delta 1 / l_{0}$, so erhält man eine mittlere Dehnung $\varepsilon_{e m}$ über diese Basislänge. Vor allem beim Stahl ergeben sich aber im gerissenen Zustand Abweichungen zwischen einer mittleren Dehnung $\varepsilon_{\text {em }}$ und der maximalen Dehnung $\varepsilon_{e, m a x}$ im Riss. Für die Umsetzung der mittleren Dehnung $\varepsilon_{\text {em }}$ in die Spannungen bzw. Kräfte im Rissquerschnitt (anhand von $\sigma-\varepsilon$-Diagrammen) wurde ein mittlerer Koeffizient $k=\varepsilon_{\mathrm{em}} / \varepsilon_{\text {e max }}$ von 0.9 angenommen.

Eine Begründung dieser Annahme wird aufgrund entsprechender Versuche in [1] gegeben. Der Koeffizient $k=0.9$ entspricht am besten der Wirklichkeit bei Dehnungen um die Fliessgrenze ( $\left.\varepsilon_{e f}\right)$. Sowohl bei kleineren als auch bei grösseren Dehnungen $\left(\varepsilon_{\theta}\right)$ nimmt $k$ oft kleinere.Werte an.

\subsubsection{Risse}

Nach jeder Laststufe wurden die in der Zugplatte von blossem Auge sichtbaren Risse mit Filzstift nachgezogen und die Rissbreiten mit einem Rissemassstab ausgemessen (Einschätzung auf $0.05 \mathrm{~mm})$. Die Rissbreiten wurden auf Klebern notiert und diese neben den Rissen fixiert. Anschliessend wurden das Rissbild und damit auch die Rissbreiten fotografisch festgehalten (Bilder 31 bis 40 ). 


\subsection{Allgemeines}

In diesem Kapitel werden die Resultate der sechs untersuchten Versuchsträger dargestellt. Vorerst seien jedoch die wichtigsten Aspekte aus den vorangegangenen Kapiteln kurz wiederholt:

Zur Erzeugung des Längsschubes $\tau_{y x}$ wurden die Träger 21 bis $Z 6$ der "Längsbelastung" $P$ unterworfen. Zur Erzeugung des Querbiegemomentes $\bar{M}$ wurden die Träger $Z 3$ bis Z6 zusätzlich noch der "Querbelastung" $\bar{P}$ (Eigenspannungszustand) unterworfen (Bild 10). Bei den Versuchsträgern $Z 3$ bis 26 wurde zudem auf der rechten Hälfte die Zugplatte in Querrichtung teilweise vorgespannt. Somit wurden die Zugplatten der Träger $Z 1$ und $Z 2$ im Anschnitt Zugplatte - Steg durch "Längsschub allein", die. Träger Z3 bis Z6 auf der linken Hälfte durch "Längsschub mit Querbiegung" und auf der rechten Hälfte durch "Längsschub mit Querbiegung und Quervorspannung" beansprucht.

Mit Ausnahme des Versuchsträgers $Z 2$ wurde die gesamte Längsbiegearmierung in die beiden Zugflanschhälften, d.h. ausserhalb des Steges, verlegt. Bei dem Träger Z2 wurden je $37.5 \%$ der Längsbiegearmierung in die Flanschhälften und $25 \%$ über dem Steg angeordnet. Bei allen Trägern wurde die Plattenquerarmierung für die Längsschubbeanspruchung nach dem Flanschfachwerk-Modell (Bild 2) konzipiert und gemäss den Gleichungen (4), (9) und (10) bemessen. Wie im Abschnitt 1.4 ausführlich beschrieben wurde und in den Bildern 3 und 4 dergestellt ist, sind folgende Merkmale wesentlich:

Beim Iräger Z1 wurde auf der linken Seite die Zugplatte um $12.5 \mathrm{~cm}$ "verkürzt", bzw. die" Querarmierung am Trägerende zusammengeschoben.

Beim Träger 22 waren $25 \%$ der Längsbiegearmierung über dem Steg angeordnet. Auf der linken Seite wurden $30 \%$ mehr Querarmierung (um 30\% erhöhte Bemessungsfliesslast) als auf der rechten Seite eingelegt.

Beim Träger 23 wurde die Plattenquerarmierung nach dem Modell "Ueberlagerung der Armierungen aus zentrisch angreifender Querzugkraft und aus Querbiegung" eingelegt.

Beim Träger 24 wurde die Plattenquerarmierung nach dem Modell "Ueberlagerung der Kräfte aus exzentrisch mit $e=z_{p} / 2$ angreifender Querzugkraft und aus Querbiegung" eingelegt.

Beim Träger 25 wurde die Plattenquerarmierung nach dem Modell "Ueberlagerung der Kräfte aus zentrisch angreifender Querzugkraft und aus Querbiegung" eingelegt.

Beim Träger 26 wurde die Plattenquerarmierung nach dem Modell "Ueberlagerung der Kräfte aus exzentrisch mit $e=z_{p} / 4$ angreifender Querzugkraft und aus Querbiegung" eingelegt.

Für die Darstellung der Versuchsresultate wurden folgende charakteristische Lastgrössen bzw. Lastkombinationen gewählt:

- Gebrauchs last der Plattenquerarmierung (1. Stufe):

$$
P_{I}=P_{I I I} / 1.8 \quad \bar{P}_{I}=\bar{P}_{I I I / 1.8}
$$

- Laststufe zwischen Gebrauchs - und Bemessungsfliesslast der Plattenquerarmierung

(2. Stufe):

$$
P_{I I}=P_{I I I} / 1.3 \quad \bar{P}_{I I}=\bar{P}_{I I I} / 1.3
$$


- Bemessungsfliesslast der Plattenquerarmierung (3. Stufe):

$$
P_{I I I}=P_{f}^{T h} \quad \bar{P}_{I I I}=\bar{P}_{f}^{T h}
$$

- Laststufen über der Bemessungsfliesslast:

$$
P>P_{I I I}
$$

Eine Besonderheit betrifft den Träger Z2: Hier wurden für die Darstellung der Versuchsresultate der linken Trägerhälfte die Laststufen entsprechend der Bemessungsfliesslast der rechten Trägerhälfte verwendet.

\subsection{Biegeverhalten der Träger}

Da bei den Versuchsträgern (Ausnahme: Träger 22) die gleiche Längsbiegearmierung eingelegt wurde, wiesen alle Träger ein ähnliches Biegeverhalten auf. Als Beispiel wurde das Biegeverhalten des Trägers 25 dargestellt.

\subsubsection{Durchbiegungen}

Bild 16 zeigt den Verlauf der Stegdurchbiegungen $\delta_{5}$. Es konnten nur relativ kleine Durchbiegungen festgestellt werden, da die zweilagige Längsbiegearmierung (ausgenommen beim Träger Z2l nie vollständig ins Fliessen kam.

\subsubsection{Stauchungen der Druckplatte}

Der Verlauf der Betonstauchungen $\varepsilon_{b}$, auf der unteren Seite der Druckplatte in Flanschmitte gemessen, ist ebenfalls in Bild 16 dargestellt. Es ist deutlich ersichtlich, dass der Verlauf der Betonstauchungen (bzw. Druckkräfte) im Vergleich zum Verlauf des Biegemomentes gegen die Trägermitte hin verschoben ist. Das Versatzmass beträgt hier $v=z / 2 \cdot \cot \alpha$.

\section{2 .3 Dehnungen der Längsbiegearmierung}

Die an einem oberen Armierungsstab gemessenen Stahldehnungen sind in Bild 17 festgehalten. Der Verlauf der Dehnungen (bzw. Zugkräfte) ist, im Vergleich zum Verlauf des Biegemomentes, gegen das Trägerende hin verschoben. Bei im Flansch angeordneter Längsbiegearmierung setzt sich das Versatzmass aus zwei Anteilen zusammen (vgl. Bild 2)

$v=z / 2 \cdot \cot \alpha+b_{e} / 2 \cdot \cot \beta$

\subsubsection{Dehnungen der Längsbiegearmierung beim Träger 22}

Beim Träger 22 (75\% der Längsarmierung in den Flanschen und $25 \%$ über dem Steg) wurden die Stahldehnungen längs einer Trägerhälfte sowohl an einem oberen Armierungsstab im Flansch (äusserer Stab) als auch an einem Armierungsstab über dem Steg (innerer Stab) gemessen und in Bild 18 dargestellt. Interessant ist, dass gegen das Trägerende hin (etwa im Bereich des Schnittes $A$ am äusseren Stab die grösseren Dehnungen gemessen wurden; d.h. der Verlauf der Dehnungen (bzw. Zugkräfte) ist, im Vergleich zum Verlauf der Dehnungen am inneren Stab, mehr gegen das Trägerende hin verschoben (in Uebereinstimmung mit dem Flanschfachwerk-Modell). Beim inneren Stab (über dem Steg) beträgt das Versatzmass nur $v=z / 2 \cdot \cot \alpha$; beim äusseren Stab (im Flansch) setzt sich das Versatzmass aus zwei Anteilen zusammen, nämlich $v=z / 2 \cdot \cot \alpha+b_{e} / 2 \cdot \cot \beta$. Gegen die Trägermitte hin fetwa ab Schnitt B) entzogen sich jedoch die äusseren Armierungsstäbe der Beanspruchung zulasten der inneren Stäbe. 
Die Dehnungen der Plattenquerarmierung der Versuchsträger $Z 1$ bis Z6, die für die Erreichung der Ziele der Untersuchung eine wichtige Rolle spielten, sind in den Bildern 19 bis 24 dargestellt. Die eingetragene Markierung "Fliessen" wurde jeweils mit $k \cdot \varepsilon_{f}$ berechnet $(k=0.9)$.

\section{3 .1 Träger $Z 1$ und $Z 2$ (Längsschub allein)}

\section{Träger Z1}

In Bild 19a ist die jeweils grösste Dehnung aus den fünf Messstrecken eines oberen Armierungsstabes sowie aus den zwei Messstrecken eines unteren Armierungsstabes aufgezeichnet.

Im Bereich II "Längsschub allein" ist erwartungsgemäss eine Zunahme der Dehnungen mit steigender Last $P$ festzustellen. Bis zur Bemessungsfliesslast $P_{\text {III }}=43$ t kamen nur vereinzelte Armierungsstäbe an den äusseren Enden der Bereiche II ins Fliessen und zwar sowohl in der oberen als auch in der unteren Armierungslage. Die Dehnungen in der zusammengeschobenen Querarmierung auf der linken Seite ("verkürzte" Zugplatte) waren grösser als auf der rechten Seite. Eine Laststeigerung auf $P=49 \mathrm{t}$ bewirkte bereits das fliessen von etwa der Hälfte aller Armierungsstäbe. Die zusammengeschobene Querarmierung kam schon bei der Bemessungsfliesslast $P_{\text {III }}$ ins Fliessen.

Im mittleren Bereich I "Keine Querbeanspruchung" blieben die gemessenen Dehnungen bis zur Höchstlast gering. Nur im Uebergangsbereich I bis II konnte mit steigender Last ein etwas grösserer Dehnungszuwachs festgestellt werden, der aber vor allem auf die "Verträglichkeitswirkung" der Flanschhälften (Scheiben) zurückzuführen ist.

In Bild 19b oben ist für die beiden Schnitte A-A und B-B (Bereich II, siehe Bild 19a) die Dehnungsverteilung quer zum Träger aufgetragen. Obwohl nach der Theorie (FlanschfachwerkModell) die Dehnungen in allen Messstrecken pro Armierungsstab und Laststufe etwa gleich sein sollten, sind relativ grosse Unterschiede festzustellen. Die Erklärung dafür ist in der unregelmässigen (willkürlichen) Rissbildung und den entsprechend variierenden gemessenen mittleren Dehnungen zu suchen.

In Bild 19b unten ist der Verlauf der maximalen Stahldehnungen in Abhängigkeit der Last $P$ in der oberen und unteren Lage der Schnitte $A-A$ und B-B aufgetragen. Die Dehnungen in der oberen und unteren Armierungslage waren nahezu gleich, was zeigt, dass das Anordnen einer zweilagigen Armierung ( $50 \%$ oben und 50\% unten) zweckmässig und gerechtfertigt ist.

\section{$\underline{\text { Träger } \mathrm{Z2}}$}

In Bild 20a ist die jeweils grösste Dehnung aus den fünf Messstrecken eines oberen Armierungsstabes sowie aus den zwei Messstrecken eines unteren Armierungsstabes aufgezeichnet.

Im Bereich II "Längsschub allein" resultierten auf der rechten Seite im Mittel etwas geringere maximale Dehnungen als beim Träger $Z 1$ (bei 22 nur 75\% der Längsbiegearmierung im Flansch bzw. Querarmierung von Z1). Erst bei einer Last von $P=56 t$ kam die Querarmierung fast über den ganzen Bereich II ins Fliessen. Die etwas geringere Querbeanspruchung (im Vergleich mit Träger 21) lässt sich vor allem dadurch erklären, dass sich die äussere Längsarmierung (für den Längsschub massgebend) mit steigender Belastung der Beanspruchung zulasten der inneren Längsarmierung entzieht (Bild 1B).

Auf der linken Seite wiesen die maximalen Dehnungen (bei $30 \%$ mehr Armierung) erwartungsgemäss wesentlich kleinere Werte auf. Die der eingelegten Querarmierung entsprechende theoretische Fliesslast betrug 1.3.P III $=56 \mathrm{t}$. Bei dieser Last kamen (wie auf der rechten Seite und wie beim Träger Z1) unter $P_{\text {III }}$ einige Armierungsstäbe am äusseren Ende des 
Bereiches II ins Fliessen.

Im mittleren Bereich I "Keine Querbeanspruchung" blieben die gemessenen Dehnungen bis zur Höchstlast auch bei diesem Träger klein. Der beim Uebergangsbereich I bis II festgestellte Dehnungszuwachs lässt sich vor allem mit der "Verträglichkeitswirkung" der Flanschhälften (Scheiben) erklären.

Bild 20b oben zeigt die Dehnungsverteilung quer zum Träger im Schnitt $B_{1}-_{1}$ der linken Trägerhälfte und im Schnitt $B_{r}-B_{r}$ der rechten Trägerhälfte. Wie beim Träger $Z 1$ wiesen die Dehnungen längs eines Armierungsstabes relativ grosse Unterschiede auf, die durch die unregelmässige Rissbildung verursacht wurden.

Bild 20b unten zeigt den Verlauf der maximalen Stahldehnungen in Abhängigkeit der Last $P$ in der oberen und unteren Lage der Schnitte $B_{1}-B_{1}$ und $B_{r}-B_{r}$. Die Dehnungen in der oberen und unteren Armierungslage waren fast gleich. Deutlich ist die geringere Dehnungszunahme im Schnitt $B_{1}-B_{1}$ zu sehen (30\% mehr Armierung).

\subsubsection{Träger Z4 (Längsschub allein, mit Querbiegung/ und Quervorspannung)}

Wie in Abschnitt 3.2 beschrieben, wurde als Variante zu den Trägern Z3, Z5 und Z6 beim Träger $Z 4$ zuerst mit der Längsbelastung $P$ bis $P_{\text {III }}$ gefahren und erst darnach die Querbelastung $\bar{P}$ stufenweise erhöht.

In Bild 21a ist die jeweils grösste Dehnung aus den fünf Messstrecken eines oberen schlaffen Armierungsstabes aufgezeichnet. Die Dehnungen der ersten drei dargestellten Laststufen (nur Längsbelastung $P$ ) wiesen einen ähnlichen Verlauf auf wie bei den Trägern 21 und 22 , obwohl die obere Querarmierung konstant über die ganze Zugplatte durchgezogen wurde. Durch das Aufbringen der Querbelastung $\bar{P}$ glichen sich die Dehnungen mit mittleren Bereich III "Reine Querbiegung" denjenigen im Bereich IV "Längsschub mit Querbiegung" etwas an.

In Bild 21b oben ist für die beiden Schnitte $B_{1}-B_{1}$ und $B_{r}-B_{r}$ im Bereich IV die Dehnungsverteilung der oberen Armierungslage quer zum Träger aufgetragen ( $B_{1}$ : schlaff armierte Seite; $B_{r}$ : teilweise vargespannte Seitel.

In Bild 21b unten ist der Verlauf der maximalen Stahldehnungen in Abhängigkeit der Last $P$ in den Schnitten $B_{1}-B_{1}$ und $B_{r}-B_{r}$ aufgetragen.

In Bild 21c sind die jeweils grössten Dehnungen aus den beiden äusseren Messstrecken a-a eines oberen schlaffen Armierungsstabes sowie aus den zwei Messstrecken eines unteren Armierungsstabes aufgezeichnet. Im mittleren Bereich III blieben die Dehnungen der unteren Armierungslage bei jeder Last fast null. Im Bereich IV hingegen nahmen sie mit steigender Längsbelastung $P z u$, um darauf mit steigender Querbelastung $\bar{P}$ (infolge QuerbiegeDruckkraft) wieder abzunehmen.

In Bild 21d ist der Verlauf der maximalen Stahldehnungen in Abhängigkeit der Last $P$ der äusseren Messstrecken a-a in den Schnitten $B_{1}-B_{1}$ und $B_{r}-B_{r}$ für die obere und untere Lage dargestellt. 


\subsubsection{Träger Z3, Z5 und Z6 (Längsschub mit Querbiegung/ und Quervorspannung}

Wie in Abschnitt 3.2 beschrieben, wurde bei den Versuchsträgern Z3, Z5 und Z6 zuerst die Querbelastung $\bar{P}$ um eine Stufe vorgefahren, mit der Längsbelastung $P$ nachgefahren und nach der dritten Stufe $\left(P_{\text {III }}\right.$, $\bar{P}_{\text {III }}$ ) nur noch mit der Längsbelastung $P$ bis zum Bruch weitergefahren. Die Träger $Z 5$ und Z6 wiesen im Gegensatz zum Träger $Z 3$ als unteré Plattenquerarmierung nur eine konstruktive Mindestarmierung auf.

\section{Träger 23}

In Bild 22a ist die jeweils grösste Dehnung aus den fünf Messstrecken eines oberen schlaffen Armierungsstabes aufgezeichnet. Ausser den ersten beiden dargestellten Laststufen ( $P_{I}, \bar{P}_{I}$ und $P_{I I}, \bar{P}_{I I}$ ) wiesen die anderen vier Leststufen die gleiche Querbelastung $\bar{P}_{\text {III }}$ auf, währenddem die Längsbelastung $P$ von $P_{I I}$ auf $P=54$ t gesteigert wurde. Im Bereich IV "Längsschub mit Querbiegung" kam die Querarmierung auf der linken Seite (schlaff armiert) bei der Bemessungsfliesslast $P_{\text {III }}$, $\bar{P}_{\text {III }}$ und auf der rechten Seite (teilweise vorgespannt) erst bei der Laststufe $P=54 \mathrm{t}$, $\bar{P}_{\text {III }}$ teilweise ins Fliessen. Im mittleren Bereich III "Reine Querbiegung" kam die Querarmierung bereits unter $P_{I I}, \bar{P}_{I I I}$ ins Fliessen.

Im Bild 22b oben ist für die beiden Schnitte $B_{1}^{-B_{1}}$ und $B_{r}{ }^{-B_{r}}$ im Bereich IV die Dehnungsverteilung der oberen Armierungslage quer zum Träger aufgetragen ( $B_{1}$ : schlaff armierte Seite: ${ }_{\Gamma}$ : teilweise vorgespannte Seite). Es ist deutlich zu erkennen, dass die Dehnungen im Schnitt $B_{\Gamma}-B_{\Gamma}$ im Vergleich zu denjenigen im Schnitt $B_{1}$ - $_{1}$ zuerst wesentlich geringer waren, sich dann aber mit steigender Belastung immer mehr anglichen.

Dieselbe Beobachtung ist auch in Bild 22b unten zu machen, wo der Verlauf der maximalen Stahldehnungen in Abhängigkeit der Last $P$ in den Schnitten $B_{1}{ }^{-B_{1}}$ und $B_{r}{ }^{-B_{r}}$ aufgetragen ist.

In Bild 22c sind die jeweils grössten Dehnungen aus den beiden äusseren Messstrecken a-a eines oberen schlaffen Armierungsstabes sowie aus den beiden Messstrecken eines unteren Armierungsstabes aufgezeichnet. Die vier dargestellten Laststufen wiesen alle die gleiche Querbelastung $\bar{P}_{\text {III }}$ auf, währenddem die Längsbelastung $P$ von $P_{\text {II }}$ auf $P=54$ gesteigert wurde. Im mittleren Bereich III blieben die Dehnungen der unteren Armierungslage sehr gering; im Bereich IV hingegen nahmen sie mit steigender Längsbelastung zu.

In Bild 22d ist der Verlauf der maximalen Stahldehnungen in Abhängigkeit der Last $P$ der äusseren Messstrecken a-a in den Schnitten $B_{1}{ }^{-B_{1}}$ und $B_{r}-B_{r}$ dargestelit.

\section{Träger 25}

In Bild 23a ist die jeweils grösste Dehnung aus den fünf Messstrecken eines oberen schlaffen Armierungsstabes aufgetragen. Ausser den ersten beiden dargestellten Laststufen ( $P_{I}$, $\bar{P}_{I}$ und $P_{I I}, \bar{P}_{I I}$ ) wiesen die anderen vier Laststufen die gleiche Querbelastung $\bar{P}_{\text {III }}$ auf, währenddem die Längsbelastung $P$ von $P_{\text {II }}$ auf $P=54$ t gesteigert wurde. Im Bereich IV "Längsschub mit Querbiegung" kam die Querarmierung auf der linken Seite (schlaff armiert) bei der Bemessungsfliesslast $P_{\text {III' }}$ P $\bar{P}_{I I}$ und auf der rechten Seite (teilweise vorgespannt) bei der Laststufe $P=49 t, \bar{P}_{I I I}$ ins Fliessen. Im mittleren Bereich III "Reine Querbiegung" kam die Querarmierung bereits unter $P_{I I}$, $\bar{P}_{\text {III }}$ ins Fliessen.

In Bild 23b oben ist für die beiden Schnitte $B_{1}-B_{1}$ und $B_{r}-B_{r}$ im Bereich IV die Dehnungsverteilung der oberen Armierungslage quer zum Träger aufgetragen ( $B_{1}$ : schlaff armierte Seite; $B_{r}$ : teilweise vorgespannte Seite).

In Bild 23b unten ist der Verlauf der maximalen Stahldehnungen in Abhängigkeit der Last $P$ in den Schnitten $B_{1}-B_{1}$ und $B_{r}{ }^{-B_{r}}$ aufgetragen. Auch hier ist deutlich zu sehen, dass die Dehnungen im Schnitt $B_{r}-B_{r}$ zuerst geringer sind, sich dann aber mit steigender Belastung denjenigen des Schnittes $B_{1}-B_{1}$ angleichen. 
In Bild $23 c$ sind die jeweils grössten Dehnungen aus den beiden äusseren Messstrecken a-a eines oberen schlaffen Armierungsstabes sowie aus den zwei Messstrecken eines unteren Armierungsstabes aufgezeichnet. Die vier dargestellten Laststufen wiesen alle die gleiche Querbelastung $\bar{P}_{\text {III }}$ auf, währenddem die Längsbelastung $P$ von $P_{\text {II }}$ auf $P=54$ t gesteigert wurde. Im Bereich IV waren die Dehnungen der unteren Armierungslage im Vergleich zu denjenigen des Trägers 23 etwas grösser, ohne jedoch (bis $P=54 t, \bar{P}_{\text {III }}$ ) zu fliessen.

In Bild 23d ist der Verlauf der maximalen Stahldehnungen in Abhängigkeit der Last $P$ der äusseren Messstrecken a-a in den Schnitten $B_{1}-B_{1}$ und $B_{r}-B_{r}$ dargestellt.

\section{Träger Z6}

In Bild $24 a$ ist die jeweils grösste Dehnung aus den fünf Messstrecken eines oberen schlaffen Armierungsstabes aufgezeichnet. Ausser den ersten beiden Laststufen $\left(P_{I}\right.$, $\bar{P}_{I}$ und $P_{I I}$, $\bar{P}_{\text {II }}$ ) wiesen die anderen vier Laststufen die gleiche Querbelastung $\bar{P}_{\text {III }}$ auf, währenddem die Längsbelastung $P$ von $P_{\text {II }}$ auf $P=54$ t gesteigert wurde. Im Bereich IV "Längsschub mit Querbiegung" kam die Querarmierung der linken Seite (schlaff armiert) schon bei der Laststufe $P_{I I}, \bar{P}_{I I I}$ und auf der rechten Seite (teilweise vorgespannt) bei der Bemessungsfliesslast $P_{\text {III }}, \bar{P}_{\text {III }}$ ins Fliessen. Obwohl bei der Bemessungsfliesslast ( $P_{\text {III }}$, $\bar{P}_{\text {III }}$ ) praktisch die ganze obere Plattenquerarmierung die Fliessdehnung erreicht hatte, konnte die Längsbelastung $P$ dennoch weiter erhöht werden, was auf eine Umlagerung der Querzugkraft $Z$ (aus Flanschfachwerk-Modell) gegen die Querbiegedruckzone hin schliessen lässt.

In Bild 24b oben ist für die beiden Schnitte $B_{1}-B_{1}$ und $B_{r}-B_{r}$ im Bereich IV die Dehnungsverteilung der oberen Armierungslage quer zum Träger aufgetragen.

In Bild 24b unten ist der Verlauf der maximalen Stahldehnungen in Abhängigkeit der Last $P$ in den beiden Schnitten $B_{1}-B_{l}$ und $B_{r}-B_{r}$ aufgetragen.

In Bild 24c sind die jeweils grössten Dehnungen aus den zwei äusseren Messstrecken a-a eines oberen schlaffen Armierungsstabes sowie aus den zwei Messstrecken eines unteren Armierungsstabes aufgezeichnet. Die vier dargestellten Laststufen wiesen alle die gleiche Querbelastung $\bar{P}_{\text {III }}$ auf, währenddem die Längsbelastung $P$ von $P_{\text {II }}$ auf $P=54$ gesteigert wurde. Die oberen Armierungsstäbe kamen auch in den äusseren Messreihen a-a zum grössten Teil schon bei der Bemessungsfliesslast ( $\left.P_{I I I}, \bar{P}_{I I I}\right)$ ins fliessen. Im Bereich IV fielen die Dehnungen der unteren Armierungslage im Vergleich zu denjenigen des Trägers 25 wesentlich grösser aus. Bei der letzten Laststufe $\bar{P}_{\text {III }}$ kamen auch die unteren Armierungsstäbe im Bereich IV zum grössten Teil ins Fliessen.

In Bild 24d ist der Verlauf der maximalen Stahldehnungen in Abhängigkeit der Last $P$ der äusseren Messstrecken a-a in den Schnitten $B_{1}-B_{1}$ und $B_{r}-B_{r}$ dargestellt. Auch hier (wie bei den Trägern 23 und 25 ) glichen sich die Dehnungen im Schnitt $B_{r}{ }^{-B_{r}}$ (teilweise vorgespannte Seite) mit steigender Belastung denjenigen im Schnitt $B_{1}{ }^{-B_{1}}$ (schlaff armierte Seite) an. 
4.4 Kräfte in der Plattenquerarmierung

\subsubsection{Träger $Z 1$ und $Z 2$ (Längsschub allein)}

Der Verlauf der Querzugkräfte $K_{0}$ in der oberen Plattenquerarmierung ist in den Bildern 25 und 26 dargestellt. Auch hier sieht man deutlich, dass der Bereich II "Längsschub allein" im Vergleich zum Querschnitt mit der Querkraft $Q$ um das Versatzmass $v$ (gemäss G1. (10)) verschoben ist. In denselben Bildern sind die Last-Querzugkraft-Diagramme $\left(K=K_{0}+K_{u}\right.$; Kräfte in der oberen und unteren Lage zusammen) des Schnittes $B_{r}{ }^{-B_{r}}$ (Z1) bzw. der Schnitte $B_{1}-B_{1}$ und $B_{r}-B_{r}(22)$ aufgezeichnet und mit theoretischen werten nach Flanschfachwerk-Modell gemäss Gl. (4) verglichen. Bei allen drei Diagrammen entsprechen die "gemessenen" Werte $K$ bei hohen Laststufen $\left(P \geq P_{I I I}\right.$ ) der Querzugkraft $Z$ nach dem Flanschfachwerk-Modell mit einem Ausbreitwinkel $B$ von $\tan \beta=0.5$ bis 0.6 . Bei kleinen Laststufen ( $P<P_{I I I}$ ) entsprechen die Werte $K$ sogar einem Ausbreitwinkel $B$ von nur $\tan \beta \cong 0.4$.

Diese Feststellungen gelten sowohl für den Fall bei dem die Längsbiegearmierung hauptsächlich in den Flanschen angeordnet war (Träger 21, Bild 25) als auch für denjenigen, wo nur ein Teil ( $F_{\mathrm{BL}}^{\mathrm{Fl}} / \mathrm{F}_{\mathrm{eL}}$ ) in den Flanschen und der Rest über dem Steg angeordnet war (Träger 22, Bild 26). Die gemachten Feststellungen gelten aber auch unabhängig von der Menge der eingelegten Plattenquerarmierung (linke und rechte Seite des Trägers $Z 2$, Bild 26), zumindest in dem hier variierten Rahmen.

\subsubsection{Träger Z4 (Längsschub allein, mit Querbiegung/ und Quervorspannung}

Längsschub allein

In Bild 27a oben ist der Verlauf der "gemessenen" Querzugkraft $K_{0}$ in der oberen Plattenquerarmierung unter der Längsbelastung $P_{I}, P_{I I}$ und $P_{I I I}$ aufgetragen.

In Bild 27a unten sind die Last-Querzugkraft-Diagramme in den beiden Schnitten $B_{1}{ }^{-B_{1}}$ und $B_{r}-B_{r}$ aufgezeichnet und mit theoretischen Werten nach Flanschfachwerk-Modell gemäss G1. (4) verglichen.

Weil die Plattenquerarmierung hauptsächlich in der oberen Lage eingelegt war, musste bei diesem Träger die Querzugkraft grösstenteils von der oberen Querarmierung aufgenommen werden. Auch hier nähern sich mit steigender Last die "gemessenen" Werte $k$ den theoretischen Werten an.

\section{Längsschub mit Querbiegung}

In Bild 27b oben ist der Verlauf der "gemessenen" Querzugkräfte (bzw. Querdruckkräfte) $K_{0}$ und $K_{u}$ in den Messstrecken a-a der oberen und unteren Querarmierung bei Laststufe $P_{I I I}, \bar{P}_{I}$ aufgetragen. Dort, wo die untere Querarmierung gedrückt war, wurde die entsprechende Druckkraft des Betons mitberücksichtigt.

Um aus $K_{0}$ und $K_{U}$ die nur durch die Längsbelastung $P$ bewirkte Querzugkraft im Flansch zu ermitteln, d.h. um die Wirkung der Querbelastung $\bar{P} z u$ eliminieren, wurde wie folgt vorgegangen:

Von der "gemessenen" Armierungskraft $K_{0}$ wurde die im Schnitt a-a (gemäss Bild 47) theoretisch ermittelte Querbiege-Zugkraft $m_{Z}=\bar{M} / z_{p}+\bar{Q} / 2 \cdot t a n \alpha$ abgezogen bzw. der "gemessenen" Kraft $K_{u}$ wurde die theoretisch ermittelte Querbiege-Druckkraft $m_{\square}=\bar{M} / z_{p}-\bar{Q} / 2 \cdot \tan \alpha$

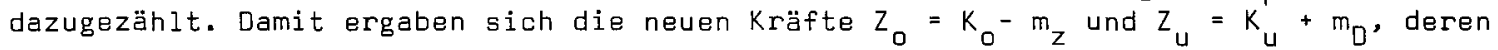
Verlauf in Bild 27b dargestellt ist. Die Summe dieser Kräfte ergibt schliesslich die allein durch die Längsbelastung $P$ bewirkte und in Bild 27b ebenfalls eingetragene Querzugkraft $z=z_{0}+z_{u}$. Die theoretisch ermittelte Querbiege-Zugkraft bzw. QuerbiegeDruckkraft im Schnitt a-a wurde unter Berücksichtigung der Neigung der Betondruckdiagona- 
len $\alpha \cong 45^{\circ}$ in der Platte für $\bar{Q}$ bestimmt.

In Bild 27b unten sind die derart ermittelten Kräfte $Z_{0}, Z_{u}$ sowie deren Summe $Z$ der Messstrecken a-a in den beiden Schnitten $B_{1}-B_{1}$ und $B_{r}-B_{r}$ für die konstante Längsbelastung $P_{\text {III }}$ in Abhängigkeit von der Last $\bar{P}$ aufgezeichnet sowie mit theoretischen Werten nach Flanschfachwerk-Modell gemäss $G 1$. (4) verglichen. Obwohl die Querbelastung $\bar{P}$ bis fast zur Laststufe $P_{I I I}, \bar{P}_{\text {III }}$ gesteigert werden konnte, konnten die entsprechenden Messungen nicht mehr vorgenommen werden. Diese Werte sind in beiden Diagrammen trotzdem eingetragen. Sie wurden aufgrund der (zutreffenden) Annahme, dass die obere und untere Querarmierung ins fliessen kam, berechnet.

Es ist deutlich zu erkennen, dass mit steigender Querbelastung $\bar{P}$ der untere QuerzugkraftAnteil $Z_{u}$ zunimmt, währenddem der obere Querzugkraft-Anteil $Z_{0}$ abnimmt. Dies deutet auf eine Verlagerung des Angriffspunktes der Querzugkraft $Z$ aus dem oberen Teil der Platte (für $\bar{P}=0$ ) in die Mitte der Platte (für $\bar{P}_{I}$ ) und schliesslich in den unteren Teil gegen die Querbiege-Druckzone hin (für $\bar{P}_{I I}$ und $\bar{P}_{\text {III }}$ ).

Auch bei diesem Träger stimmt die gesamte Querzugkraft $Z$ sowohl auf der schlaff armierten Seite (Schnitt $B_{1}-B_{I}$ ) als auch auf der teilweise vorgespannten Seite (Schnitt $B_{r}-B_{r}$ ) mit steigender Last bzw. fortschreitender Rissbildung immer besser mit den theoretischen Werten gemäss Flanschfachwerk-Modell mit einem Ausbreitwinkel $\beta$ von $\tan \beta=0.5$ bis 0.6 überein.

\section{4 .3 Träger $23, Z 5$ und Z6 (Längsschub mit Querbiegung/ und Quervorspannung)}

\section{Träger $Z 3$}

In Bild 28a oben ist der Verlauf der "gemessenen" Querzugkraft $K_{0}$ in der oberen Plattenquerarmierung dargestellt. Ausser den ersten zwei dargestellten Laststufen $\left(P_{I}, \bar{P}_{I}\right.$ und $P_{I I}, \bar{P}_{I I}$ ) weisen die vier folgenden Laststufen die gleiche Querbelastung $\bar{P}_{\text {III }}$ auf, wobei die Längsbelastung $P$ von $P_{I I}$ auf $P=54$ t gesteigert wurde. Bei der letzten Laststufe $\left(P=54 t, \bar{P}_{I I I}\right)$ wurde praktisch auf der ganzen Länge des Trägers der Fliesswiderstand der oberen Querarmierung erreicht.

In Bild 28a unten ist die Querzugkraft $K_{0}$ der oberen Querarmierung in den beiden Schnitten $B_{I}-B_{1}$ und $B_{r}-B_{r}$ in Abhängigkeit der Last $P$ dargestellt.

In Bild $28 b$ oben ist der Verlauf der "gemessenen" Querzugkräfte (bzw. Querdruckkräfte) $K_{0}$ und $K_{u}$ in den Messstrecken a-a der oberen und unteren Querarmierung bei der Bemessungsfliesslast $P_{\text {III }}, \bar{P}_{\text {III }}$ aufgetragen. Dort, wo die untere Querarmierung gedrückt war, wurde die entsprechende Betondruckkraft mitberücksichtigt. Um aus $K_{D} u n d k_{u} d i e$ nur durch die Längsbelastung $P$ bewirkte Querzugkraft im Flansch zu ermitteln, d.h. um die Wirkung der Querbelastung $\bar{P}$ zu eliminieren, wurde auch hier wie bei $Z 4$ vorgegangen: Von der "gemessenen" Armierungskraft $K_{0}$ wurde die im Schnitt a-a (gemäss Bild 47) theoretisch ermittelte Querbiege-Zugkraft $m_{z}=\bar{M} / z_{p}+\bar{Q} / 2 \cdot t a n \alpha$ abgezogen, bzw. der "gemessenen" Kraft $K_{u}$ wurde die theoretisch ermittelte Querbiege-Druckkraft $m_{D}=\bar{M} / z_{0}-\bar{Q} / 2 \cdot \tan \alpha$ dazugezählt. Damit ergaben sich die neuen $k$ räfte $z_{0}=k_{0}-m_{z}$ und $z_{u}=k_{u}+m_{0}$, deren Verlauf in Bild 28b dargestellt ist. Die Summe dieser Kräfte ergibt schliesslich die allein durch die Längsbelastung $P$ bewirkte und in Bild 28b ebenfalls aufgetragene Querzugkraft $z=z_{0}+z_{u}$. Die theoretisch ermittelte Querbiege-Zugkraft im Schnitt a-a wurde unter Berücksichtigung der Neigung der Betondruckdiagonalen $\alpha \cong 45^{\circ}$ in der Platte für $\bar{Q}$ bestimmt.

In Bild 28b unten sind die derart ermittelten Kräfte $Z_{0}$, $Z_{u}$ sowie deren Summe $Z$ der Messstrecken a-a in den beiden Schnitten $B_{1}-B_{1}$ und $B_{r}-B_{r}$ in Abhängigkeit von der Last $P$ aufgezeichnet sowie mit theoretischen Werten nach Flanschfachwerk-Modell gemäss Gl. (4) verglichen. 
Mit steigender Last $P$ nähern sich die stets etwas kleineren Werte $Z_{0}$ denjenigen von $Z_{u}$ an. Dies deutet auf eine gewisse Verlagerung des Angriffspunktes der Querzugkraft $Z$ aus dem unteren Teil der Platte gegen die Mitte hin.

Auch bei diesem Träger stimmt die gesamte Querzugkraft $Z$ sowohl auf der schlaff armierten Seite (Schnitt $B_{1}-B_{l}$ ) als auch auf der teilweise vorgespannten Seite (Schnitt $B_{r}-B_{r}$ ) mit steigender Last bzw. fortschreitender Rissbildung immer besser mit den theoretischen Werten gemäss Flanschfachwerk-Modell mit einem Ausbreitwinkel $\beta$ von $\tan \beta=0.5$ bis 0.6 überein.

\section{Träger 25}

In Bild 29a oben ist der Verlauf der "gemessenen" Querzugkraft $K_{0}$ in der oberen Plattenquerarmierung dargestellt. Ausser den ersten zwei dargestellten Laststufen $\left(P_{I}\right.$, $\bar{P}_{I}$ und $P_{I I}, \bar{P}_{I I}$ ) weisen die vier folgenden Laststufen die gleiche Querbelastung $\bar{P}_{\text {III }}$ auf, wobei die Längsbelastung $P$ von $P_{\text {II }}$ auf $P=54$ gesteigert wurde. Bei der letzten Laststufe $\left(P=54 t, \bar{P}_{I I I}\right)$ wurde praktisch auf der ganzen Länge des Trägers der Fliesswiderstand der oberen Querarmierung erreicht.

In Bild 29a unten ist die Querzugkraft $K_{0}$ der oberen Querarmierung in den beiden Schnitten $B_{1}-B_{1}$ und $B_{r}-B_{r}$ in Abhängigkeit der Last $P$ dargestellt.

Die Beanspruchung der oberen Querarmierung war praktisch gleich derjenigen des Trägers 23 (gleiche obere Querarmierung).

In Bild 29b oben ist der Verlauf der "gemessenen" Querzugkräfte (bzw. Querdruckkräfte) $K_{0}$ und $K_{u}$ in den Messstrecken a-a der oberen und unteren Querarmierung bei der Bemessungsfliesslast $P_{\text {III. }}$, $\bar{P}_{\text {II }}$ aufgetragen. Dort, wo die untere Querarmierung gedrückt war, wurde die Betondruckkraft mitberücksichtigt. Auch hier wurden wiederum (wie bei den Trägern 23 und $z 4$ ) die $k_{r} a ̈ f t e z_{0}=k_{0}-m_{z}$ und $z_{u}=k_{u}+m_{0}$ bestimmt und deren Verlauf in Bild 29b dargestellt. Die Summe dieser Kräfte ergibt schliesslich die allein durch die Längsbelastung $P$ bewirkte und in Bild 29b ebenfalls eingetragene Querzugkraft $z=Z_{0}+Z_{u}$.

Im Vergleich zum Träger 23 wies die untere Querzugkraft (bzw. Querdruckkraft) $k_{u}$ in den Bereichen IV "Längsschub mit Querbiegung" ca. $50 \%$ kleinere Werte auf (bei ca. $60 \%$ weniger unterer Querarmierung\}.

In Bild 29b unten sind die ermittelten Kräfte $Z_{0}, Z_{u}$ sowie deren Summe $Z$ der Messstrecken a-a in den beiden Schnitten $B_{1}{ }^{-B_{1}}$ und $B_{r}-B_{r}$ in Abhängigkeit der Last $P$ aufgezeichnet sowie mit theoretischen Werten nach Flanschfachwerk-Modell gemäss Gl. (4) verglichen.

Mit steigender Last $P$ nimmt der anfangs kleinere Wert $Z_{0}$ stärker zu als der (im Vergleich zum Träger 23 kleinere) Wert $Z_{u}$. Etwa nach Erreichen der Bemessungsfliesslast ( $P_{\text {III }}$, $\bar{P}_{\text {III }}$ ) weisen beide Werte ähnliche Grössen auf. Dies deutet auf eine Verlagerung des Angriffspunktes der Querzugkraft $z$ aus dem unteren Teil zur Mitte der Platte hin.

Auch bei diesem Träger stimmt die gesamte Querzugkraft $Z$ sowohl auf der schlaff armierten Seite (Schnitt $B_{1}-B_{1}$ ) als auch auf der teilweise vorgespannten Seite (Schnitt $B_{r}-B_{r}$ ) mit steigender Last bzw. fortschreitender Rissbildung immer besser mit den theoretischen Werten gemäss Flanschfachwerk-Modell mit einem Ausbreitwinkel $\beta$ von $\tan \beta=0.5$ bis 0.6 überein.

\section{Träger 26}

In Bild 30a oben ist der Verlauf der "gemessenen" Querzugkraft $K_{0}$ in der oberen Plattenquerarmierung dargestellt. Ausser den ersten zwei dargesteliten Laststufen $\left(P_{I}, \bar{P}_{I}\right.$ und 
$P_{\text {II }}, \bar{P}_{I I}$ ) weisen die folgenden Laststufen die gleiche Querbelastung $\bar{P}_{\text {III }}$ auf, wobei die Längsbelastung $P$ von $P_{I I}$ auf $P=54$ t gesteigert wurde. Bei der Bemessungsfliesslast ( $P_{\text {III, }} \bar{P}_{\text {III }}$ ) wurde auf der ganzen Länge des Trägers der Fliesswiderstand der oberen Querarmierung erreicht.

In Bild 30a unten ist die Querzugkraft $K_{0}$ der oberen Querarmierung in den beiden Schnitten $B_{1}-B_{2}$ und $B_{r}-B_{F}$ in Abhängigkeit der Last $P$ dargestellt.

Die im Vergleich zu den Trägern $Z 3$ und 25 in den Bereichen IV "Längsschub mit Querbiegung" um ca. $20 \%$ verringerte obere Querarmierung bewirkte ein relativ frühes Fliessen derselben. Bis zum Fliessbeginn wiesen die Querzugkräfte $k_{0}$ jedoch ähnliche Werte auf wie bei den Trägern $Z 3$ und $Z 5$.

In Bild 30b oben ist der Verlauf der "gemessenen" Querzugkräfte (bzw. Querdruckkräfte) $K_{0}$ und $K_{u}$ in den Messstrecken a-a der oberen und unteren Querarmierung bei der Bemessungsfliesslast $P_{I I I}, \bar{P}_{I I I}$ aufgetragen. Dort, wo die untere Querarmierung gedrückt war, wurde die Betondruckkraft mitberücksichtigt. Auch hier wurden wiederum (wie bei Z3, Z4 und Z5) die kräfte $z_{0}=k_{0}-m_{z}$ und $z_{u}=k_{u}+m_{0}$ bestimmt und deren Verlauf in Bild 30b dargestellt. Die Summe dieser Kräfte ergibt schliesslich die allein durch die Längsbelastung bewirkte und in Bild 30b ebenfalls eingetragene Querzugkraft $Z=z_{0}+z_{u}$.

In Bild 30b unten sind die ermittelten Kräfte $Z_{0}, Z_{u}$ sowie deren Summe $Z$ der Messstrecken a-a in den beiden Schnitten $B_{1}-B_{1}$ und $B_{r}-B_{r}$ in Abhängigkeit von der Last $P$ aufgezeichnet sowie mit theoretischen Werten nach Flanschfachwerk-Modell gemäss G1. (4) verğlichen.

Im Vergleich mit dem Träger $Z 5$ wiesen die Querzugkraft-Anteile $Z_{0}$ und $Z_{u}$ bis zur Bemessungsfliesslast ( $P_{I I I}, \bar{P}_{I I I}$ ) ähnliche Werte auf. Bei einer weiteren Laststeigerung $P>P_{\text {III }}$ konnte der obere Querzugkraft-Anteil $Z_{0}$ nicht mehr vergrössert werden (Fliesswiderstand erreicht). Bei diesem Träger blieb der Angriffspunkt der Querzugkraft $Z$ im unteren Teil der Platte. Durch eine Verkleinerung des theoretischen Ausbreitwinkels $\beta$ (auf $\tan \beta \leq 0.5$ ) konnte die Last trotz Fliessens der oberen Armierung noch bis auf $P=$ $54 \mathrm{t}, \bar{P}_{\text {III }}$ gesteigert werden. Auch bei diesem Träger konnte kein Unterschied in der Querzugkraft zwischen der linken (schlaff armierten) und der rechten (teilweise vorgespannten) Seite festgestellt werden.

4.5 Verlagerung des Angriffspunktes der Querzugkraft aus Längsschub

In Tabelle 15 sind für sämtliche Träger die Versuchsresultate der Messstrecken a-a in beiden Schnitten $B_{1}^{-B_{1}}$ (schlaff armierte Seite) und $B_{r}-B_{r}$ (teilweise vorgespannte Seite) zusammengestellt.

Es wurden folgende Grössen berechnet und eingetragen:

K : "gemessene" Querzugkraft $\left(K_{0}+K_{u}\right)$ in der Querarmierung (Träger 21 und 22 )

$Z \quad$ : durch die Längsbelastung $P$ bewirkte Querzugkraft

$$
\left(z=z_{0}+z_{u}, \quad z_{0}=k_{0}-m_{Z}, \quad z_{u}=k_{u}+m_{D}\right)
$$

$\tan \beta$ : experimentell bestimmter Ausbreitwinkel nach Gl. (4)

$\tau \quad$ : Längsschubspannung $\tau_{y x}$ nach GI. (14)

e : "gemessene" Exzentrizität des Angriffspunktes der Querzugkraft Z

$e_{\text {max }}$ : maximal anzusetzende Exzentrizität nach 61. (23) im Anhang

e/e ${ }_{\max }$ : Verhältnis zwischen "gemessener" Exzentrizität e und $8_{\max }$ 
In Bild 50 wurde die Verlagerung des Angriffspunktes der Querzugkraft aus Längsschub in den Messstrecken a-a in beiden Schnitten $B_{1}-B_{1}$ und $B_{r}-B_{r}$ der Träger 23 bis Z6 graphisch dargestellt und mit dem Wert gemäss dem theoretischen Ansatz für e max nach G1. (23) verglichen.

\subsubsection{Längsschub allein, gleiche obere und untere Querarmierung}

Bei den Trägern $Z 1$ und $Z 2$ wurde die obere und die untere Querarmierung etwa gleich stark beansprucht. Die Querzugkraft griff etwa in Plattenmitte an, entsprechend dem der Bemessung der Querarmierung zugrundegelegten Modell (e $\cong 0$ ).

\subsubsection{Längsschub allein, ungleiche obere und untere Querarmierung}

Eine hauptsächlich einlagig angeordnete Querarmierung (Träger Z4, anfängliche Prüfung auf Längsschub allein) bewirkte eine Verlagerung des Angriffspunktes der Querzugkraft zur vorhandenen Querarmierung hin $\left(e \rightarrow-z_{p} / 2\right)$.

Sofern die Exzentrizität e der Querzugkraft $Z$ den Wert gemäss dem theoretischen Ansatz für $e_{\text {max }}$ nach [1. (23) im Anhang nicht (wesentlich) übersteigt, ist grundsätzlich keine Verminderung der Tragfähigkeit zu erwarten.

\subsubsection{Längsschub mit Querbiegung, gleiche oder ungleiche obere und untere Armierung}

Beim Träger 24 (anfängliche Prüfung auf Längsschub allein) bewirkte das Aufbringen des Querbiegemomentes $\bar{M}$ eine Verlagerung der Querzugkraft von der oberen zur unteren Plattenseite hin, entsprechend dem der Bemessung der Querarmierung zugrundegelegten Modell $\left(e \rightarrow+z_{p} / 2\right)$.

Bei den Trägern Z3, 25 und Z6 (Querbelastung $\bar{P}$ jeweils um eine Stufe vorgezogen) bewirkte das Querbiegemoment $\bar{M}$ anfänglich eine Verlagerung der Querzugkraft gegen die (steifere) Querbiege-Oruckzone hin. Mit dem Steigern der Längsbelastung $P$ verlagerte sich die Querzugkraft bei 23 und $Z 5$ jedoch immer mehr gegen die Plattenmitte hin, entsprechend den der Bemessung der Querarmierung zugrundegelegten Modellen $(e \rightarrow \pm 0)$.

Beim Träger Z6 verlagerte sich die Querzugkraft nach dem Fliessen der oberen Querarmierung (ca. ab der Bemessungsfliesslast $P_{\text {III }}, \bar{P}_{\text {III }}$ ) wieder gegen die Querbiege-Druckzone hin, entsprechend dem der Bemessung der Querarmierung zugrundegelegten Modell $\left(e++z_{p} / 4\right)$.

\subsection{Rissverhalten}

\subsubsection{Träger $Z 1$ und $Z 2$}

Die Bilder 31 und 33 zeigen die Entwicklung der Rissbilder. Zwei Arten von Rissen sind ersichtlich: "Biegerisse", die senkrecht zur Trägeraxe verlaufen, und "Schubrisse", die von der Flanschmitte aus schräg zur Längsbiegearmierung verlaufen. Im Bereich der Trägermitte verlaufen die "Schubrisse" relativ steil, gegen das Trägerende hin jedoch immer flacher, d.h. mit kleinerer Neigung zur Trägeraxe.

\section{Träger 21}

In Bild 32 oben ist der Verlauf der grössten Rissbreiten längs der Zugplatte bei der Bemessungsfliesslast $P_{\text {III }}$ dargestellt.

In Bild 32 unten ist für die linke und rechte Seite des Trägers der Last-RissbreitenVerlauf der grössten "Schubrisse" im Bereich II aufgetragen. Es konnten bei beiden Seiten (gleiche Querarmierung) ähnliche Rissbreiten festgestellt werden. Bei der Gebrauchslast $P_{I}$ betragen die grössten Rissbreiten 0.25 bzw. $0.20 \mathrm{~mm}$ und bei der Bemessungsfliess- 
last $P_{\text {III }} 0.40$ bzw. $0.45 \mathrm{~mm}$.

\section{Träger Z2}

Bild 34 oben zeigt den Verlauf der grössten Rissbreiten längs der Zugplatte bei der Bemessungsfliesslast PIII. Auf der linken Seite (30\% mehr Querarmierung) weisen die "Schubrissen erwartungsgemäss wesentlich kleinere Breiten auf als jene auf der rechten Seite.

In Bild 34 unten ist für die linke und rechte Seite des Trägers der Last-RissbreitenVerlauf der grössten "Schubrisse" im Bereich II dargestellt. Die grössten Rissbreiten betragen bei der Gebrauchslast $P_{I}$ auf der linken Seite $0.15 \mathrm{~mm}$ und auf der rechten Seite $0.25 \mathrm{~mm}$. Bei der Bemessungsfliesslast $P_{\text {III }}$ ergaben sich Rissbreiten von $0.35 \mathrm{~mm}$ links und $0.55 \mathrm{~mm}$ rechts.

\subsubsection{Träger 24}

Bild 35 zeigt die Entwicklung der Rissbilder.

In Bild 36 oben ist der Verlauf der grössten Rissbreiten längs der Zugplatte bei der Laststufe $P_{\text {III }}, \bar{P}_{0}$ dargestellt.

In Bild 36 unten ist für die linke und rechte Seite des Trägers der Last-RissbreitenVerlauf der grössten "Schubrisse" im Bereich IV aufgetragen. Auf der rechten Seite bewirkte die teilweise Quervorspannung sowohl eine höhere Risslast von $18 \mathrm{t}(12 \mathrm{t}$ auf der linken Seite) als auch wesentlich kleinere Rissbreiten im Gebrauchslast-Bereich.

\subsubsection{Träger Z3, Z5 und Z6}

Die Bilder 37 und 39 zeigen die Entwicklung der Rissbilder der Träger 25 und Z6. Die Rissbilder und der Rissverlauf des Trägers 23 sind fast gleich wie beim Träger $Z 5$ und wurden deshalb nicht dargestellt. Bei allen drei Trägern (wie auch bei $Z 4$ mit $\bar{P}>\bar{P}_{0}$ ) konnten die Risse infolge der zusätzlichen Querbiegebeanspruchung nicht mehr eindeutig nach Rissarten unterschieden werden.

\section{Träger 25}

In Bild 38 oben ist der Verlauf der grössten Rissbreiten längs der Zugplatte bei vier charakteristischen Laststufen aufgetragen.

Bild 38 unten zeigt für die linke und rechte Seite des Trägers den Last-RissbreitenVerlauf der grössten Risse im Bereich IV. Die Rissbreiten auf der linken Seite (schlaff armiert) sind im Vergleich zu denjenigen auf der rechten Seite (teilweise vorgespannt) im Gebrauchslast-Bereich etwas grösser. Bei der Gebrauchslast $P_{I}, \bar{P}_{I}$ betragen die grössten Rissbreiten $0.15 \mathrm{~mm}$ links und $0.10 \mathrm{~mm}$ rechts. Bei der Bemessungsfliesslast PIII, PIII ergaben sich Rissbreiten von $0.45 \mathrm{~mm}$ links und ebenfalls $0.45 \mathrm{~mm}$ rechts.

\section{Träger 26}

Bild 40 oben zeigt den Verlauf der grössten Rissbreiten längs der Zugplatte bei vier charakteristischen Laststufen.

In Bild 40 unten ist für die linke und rechte Seite des Trägers der Last-RissbreitenVerlauf der grössten Risse im Bereich IV aufgetragen. Auch hier sind die Risse auf der linken Seite (schlaff armiert) bis zur Bemessungsfliesslast $P_{\text {III. }}$ P $\bar{P}_{\text {II }}$ grösser als auf der rechten Seite (teilweise vorgespannt). Bei der Gebrauchslast $P_{I}$, $\bar{P}_{I}$ betragen die grössten Rissbreiten $0.25 \mathrm{~mm}$ links und $0.15 \mathrm{~mm}$ rechts. Bei der Bemessungsfliesslast P III, $\bar{P}_{\text {III }}$ ergaben sich Rissbreiten von $0.50 \mathrm{~mm}$ links und ebenfalls $0.50 \mathrm{~mm}$ rechts. 
Die in Querrichtung teilweise vorgespannten Zugplatten zeigten im Vergleich mit den nur schlaff armierten Zugplatten ein günstigeres Rissverhalten. Zum Beispiel entstanden beim Träger Z6 die ersten, nicht senkrecht zur Trägeraxe verlaufenden Risse ("Schubrisse") auf der vorgespannten Seite (rechts) erst bei $\sim P_{I} / 2, \bar{P}_{I}$. Dies entspricht bei $\gamma=z \cdot d / \bar{M} \cong 0.9$ einem effektiven Vorspanngrad $K^{\prime}=M_{z u l,} V^{/ M_{g+p}}$ von 1.0 (siehe.Abschnitt 1.4.2) und somit einer "effektiven" Randzugspannung bei der Rissbildung $\sigma_{r}$ von $\sim 33 \mathrm{~kg} / \mathrm{cm}^{2}$. Auf der schlaff armierten Seite (links) entstanden die ersten Risse schon beim Steigern der Querbelastung $\bar{P}$ bei $\sim P_{0}, 3 \bar{P}_{I} / 4$, was bei $\gamma=0$ einem effektiven Vorspanngrad $k$ 'von 0.75 und somit einer "effektiven" Randzugspannung bei der Rissbildung or von $\sim 37 \mathrm{~kg} / \mathrm{cm}^{2}$ entspricht.

Bei einem Bauwerk dürften zum Beispiel infolge Schwind- und Temperaturzwängungen, oft wiederholter Belastungen, usw. nicht derart hohe Randzugspannungen $\sigma_{r}$ auftreten. Trotzdem hat die teilweise Vorspannung zwei wichtige Vorteile: erstens wird die Risslast erhöht und zweitens treten im Gebrauchslast-Bereich schmälere Risse auf als bei einem analogen nur schlaff armierten Bauteil.

\subsection{Bruchverhalten}

\subsubsection{Träger $\mathrm{Z1}$ und $\mathrm{Z} 2$}

Bild 41 zeigt die Bruchbilder des Trägers 21. Der Bruch (Schubbruch) erfolgte beim linken Trägerende, wo die Zugplatte durch die zusammen geschobene Querzugarmierung "verkürzt" war, bei der Last $P_{U}=52 \mathrm{t}$. Anhand der Bruchbilder ist deutlich zu sehen wie wichtig es ist, die Querzugarmierung bis um das Versatzmass v "hinter" der konzentrierten krafteinleitung einzulegen.

Der Träger 22 versagte bei der Last $P_{u}=58 \mathrm{t}$ durch Biegebruch. Bis Erreichen der Biegetragfähigkeit $\left(P_{U B}=58 \mathrm{t}\right)$ konnte kein Bruch im Flansch erzielt werden, obwohl aus den gemessenen Rissbreiten geschlossen werden konnte, dass bei nur wenig höherer Last vermutlich ein ähnlicher Bruch wie beim Träger 21 erfolgt wäre.

\subsubsection{Träger 24}

Bild 42 zeigt die Bruchbilder des Trägers 24. Das Versagen erfolgte nahezu bei der Bemessungsfliesslast $P_{I I I}, \bar{P}_{I I I}$, mit $\bar{P}_{U} \leq \bar{P}_{I I I}$ durch einen kombinierten Schub- und Biegebruch längs der schlaff armierten Zugplatte der linken Trägerhälfte. Die Bruchfläche verläuft relativ flach vom Stegansatz (unten) bis zu der im Flansch eingelegten Längsarmierung (oben).

\subsubsection{Träger $Z 3, Z 5$ und $Z 6$}

Die Bilder 43 bis 45 zeigen die Bruchbilder der Träger Z3, 25 und Z6. Alle drei Träger wiesen praktisch dasselbe Bruchverhalten wie der Träger 24 auf.

Beim Träger 23 erfolgte der Bruch längs der schlaff armierten Zugplatte der linken Trägerhälfte bei $P_{u}=58 \mathrm{t}, \bar{P}_{I I I}$. Die Bruchfläche verläuft ebenfalls flach vom Stegansatz (unten) bis zu der im Flansch eingelegten Längsarmierung (oben).

Beim Träger 25 erfolgte der Bruch gleichzeitig in beiden Flanschhälften längs der teilweise vorgespannten Zugplatte der rechten Trägerhälfte bei $P_{u}=58 \mathrm{t}, \bar{P}_{I I I}$. Die beiden fast symmetrischen Bruchflächen verlaufen flach vom Stegansatz (unten) bis zu den im Flansch eingelegten Längsarmierungen. 
armierten Zugplatte der linken Trägerhälfte bei $P_{U}=56 t, \bar{P}_{I I I}$. Auch hier verlaufen die beiden fast symmetrischen Bruchflächen flach vom Stegansatz (unten) bis zu den im Flansch eingelegten Längsarmierungen (oben). 
5. FOLGERUNGEN

Nachstehend werden die wichtigsten Folgerungen aus den beschriebenen Versuchen (unter Hinweis auf entsprechende Bilder) wiederholt und zusammenfassend dargestellt.

5.1 Längsschub allein

\subsubsection{Tragfähigkeit}

Die Zugkraft $K$ in der Plattenquerarmierung (Bilder 25 und 26 ) nähert sich mit steigender Längsbelastung $P$ der Querzugkraft $Z$ gemäss Flanschfachwerk-Modell (Bild 2)

$$
Z=\frac{Q}{2 \cdot z} \cdot \frac{F_{e L}^{F I}}{F_{e L}} \tan \beta
$$

mit dem Ausbreitwinkel $\beta$ von

$$
\tan \beta=0.5 \text { bis } 0.6
$$

Die Formeln (4) und (16) wurden bestätigt für die beiden Fälle, wo

- die gesamte Längsarmierung $\left(F_{e L}^{F l} / F_{e L} \cong 1\right.$, Träger $\left.Z 2\right)$

- nur ein Teil der Längsarmierung $\left(F_{e L}^{F l} / F_{e L}<1\right.$, Träger 22)

ausserhalb des Steges im Zugflansch liegt. Beim zweiten Fall dürften diese Formeln mehr oder weniger auf der sicheren Seite liegen.

Das für die Anordnung der Querarmierung verwendete Versatzmass

$$
v \cong 0.3 \cdot\left(z+2 \cdot b_{e}\right)
$$

hat sich als zweckmässig erwiesen.

Eine Verkürzung des Versatzmasses $v$ mit Zusammenschieben der entsprechenden Querarmierung bei der Lasteinleitung gemäss Bild 49 wirkt sich ungünstig aus (Träger Z1, linke Seite). Eine solche Reduktion bewirkt eine lokale Vergrösserung des Ausbreitwinkels und damit der Querzugkraft.

Das Versatzmass $\vee$ nach Gl. (10) sollte daher wenn möglich im Bereich konzentrierter Lasteinleitungen auf der Zugflanschseite des Trägers nicht unterschritten werden. Andernfalls ist die Querarmierung entsprechend zu verstärken.

Die Längsarmierung muss in jedem Fall "hinter" dem Versatzmass einwandfrei verankert werden.

Eine hauptsächlich einlagig angeordnete Querarmierung (Träger 24, Bilder 27a und 50) bewirkt eine Verlagerung des Angriffspunktes der Querzugkraft zur vorhandenen Querarmierung hin.

Die Beanspruchung in den beiden Lagen der Querarmierung entspricht den Kräften

$$
K_{0, u}=\frac{z}{2}\left(1 \mp \frac{2 \cdot e}{z_{p}}\right)
$$

(Ein Vorschlag für die bei einer Bemessung maximal anzusetzenden Exzentrizität e wird im Anhang gemacht.) 


\section{1 .2 Verhalten im Gebrauchszustand}

Unter Berücksichtigung der kleinen Durchmesser und Abstände der eingelegten Armierungsstäbe sind die in den Versuchen festgestellten maximalen Rissbreiten beträchtlich.

Bei einer Bemessung der Querarmierung gemäss Gl. (4) mit $\tan \beta=0.5$ bis 0.6 (anstatt mit $\tan \beta=0.66$ ) sind bei der entsprechenden Gebrauchslast "Schubrissbreiten" bis zu $0.30 \mathrm{~mm}$ zu erwarten (Bilder 32 und 34 ).

\subsection{Längsschub mit Querbiegung}

\subsubsection{Tragfähigkeit}

Die Querzugkraft $Z\left(Z_{0}+Z_{u}\right)$ aus Längsschub allein (Bilder 27b bis 30b, linke Seite, bzw. Schnitt $B_{1}-B_{1}$ ) nähert sich mit steigender Längsbelastung der theoretischen Querzugkraft gemäss Flanschfachwerk-Modell bzw. gemäss den Gleichungen (4) und (16).

Eine Verlagerung des Angriffspunktes der Querzugkraft $Z$ gegen die Biegedruckzone hin ist möglich ( $v$ gl. Bild 50 ).

Die Beanspruchung in den beiden Lagen der Querarmierung entspricht den Kräften

$$
k_{0, u}=\frac{z}{2}\left(1 \mp \frac{2 \cdot e}{z_{p}}\right) \pm \frac{M}{z_{p}}
$$

(Ein Vorschlag für die bei einer Bemessung maximal anzusetzenden Exzentrizität e wird im Anhang gemacht.)

\subsubsection{Verhalten im Gebrauchszustand}

Der Angriffspunkt der angreifenden Querzugkraft $Z$ wird erst gegen das Fliessen der Querarmierung hin vom Bemessungsmodell beeinflusst (Bild 50).

Bei einer Bemessung der Querarmierung unter Berücksichtigung einer Verlagerung des Angriffspunktes der Querzugkraft mit den Kräften gemäss Gl. (8) sind bei der Gebrauchslast grössere Rissbreiten in Kauf zu nehmen als bei einer Bemessung mit zentrisch angreifender Querzugkraft (Bilder 38, 40).

\subsection{Längsschub mit Querbiegung und Quervorspannung}

\subsubsection{Tragfähigkeit}

Die Querzugkraft $Z\left(Z_{0}+Z_{u}\right)$ aus Längsschub allein (Bilder 27b bis 30b, rechte Seite, bzw. Schnitt $B_{r}-B_{r}$ ) nähert sich auch hier mit steigender Längsbelastung der theoretischen Querzugkraft gemäss Flanschfachwerk-Modell, resp. gemäss den Gleichungen (4) und (16).

Eine Verlagerung des Angriffspunktes der Querzugkraft $Z$ gegen die Biegedruckzone ist auch hier möglich (vgl. Bild 50). Dies bedeutet, dass auch hier dieselben, für Längsschub und Querbiegung abgeleiteten Gleichungen gelten. Für die Tragfähigkeit ist allein der Fliesswiderstand der gesamten schlaffen und vorgespannten Querarmierung massgebend. 


\subsubsection{Verhalten im Gebrauchszustand}

Eine teilweise Quervorspannung der Zugflanschen bewirkt kleinere Rissbreiten als nur eine schlaffe Armierung (Bilder 36, 38, 40). Erst oberhalb der Gebrauchslast - was für die Praxis allerdings nicht wesentlich ist - gleichen sich die Rissbreiten denjenigen von analogen, nur schlaff armierten Zugflanschen an. 
In Druck-bzw. Zugflanschen dünnwandiger Plattenbalken- und Hohlkastenquerschnitte treten ausser Längsdruck bzw. Längszug häufig auch Längsschub und Querbiegung auf.

In [1] und [2] ist über Versuche an Druckflanschen von sieben Ștahlbetonträgern berichtet worden.

Gegenstand des vorliegenden Versuchsberichtes ist die Problematik in Zugflanschen. Es sollten die Grundlagen für die Entwicklung einfacher, mit der Wirklichkeit gut übereinstimmender Bemessungs-Modelle bereitgestellt werden. In den Jahren 1977 und 1978 wurden am Institut für Baustatik und Konstruktion der Eidgenössischen Technischen Hochschule Zürich Versuche an sechs I-Trägern durchgeführt.

Der Bemessung der Plattenquerarmierung wurden grundsätzlich die folgenden Modelle zugrundegelegt (Bild 3):

\section{Längsschub allein}

Träger 21, Z2: Flanschfachwerk-Modell (Bild 2)

\section{Längsschub mit Querbiegung/ und Quervorspannung}

Träger 23 : Ueberlagerung der Armierungen aus zentrisch angreifender Querzugkraft und aus Querbiegung

Träger 25 : Ueberlagerung der Kräfte aus zentrisch angreifender Querzugkraft und aus Querbiegung

Träger Z4, Z6: Ueberlagerung der Kräfte aus exzentrisch angreifender Querzugkraft und aus Querbiegung

In der rechten Trägerhälfte von $Z 3$ bis $Z 6$ wurde die Zugplatte in Querrichtung teilweise vorgespannt.

Die Längsbiegearmierung wurde je zur Hälfte in beiden Flanschhälften angeordnet mit Ausnahme des Trägers 22 . Hier wurden nur $75 \%$ der Längsarmierung im Flansch angeordnet, die restlichen $25 \%$ wurden über dem Steg eingelegt.

Alle übrigen Parameter wie Trägerabmessungen, Querkraftarmierung des Steges, Druckflanscharmierung, Betonqualität, usw. wurden konstant gehalten (Bild 5).

Aufgrund der vorliegenden Versuchsergebnisse und den in Kapitel 5 dargestellten Folgerungen wird für die Bemessung der Armierungen in Zugflanschen für Längsschub mit Querbiegung und Quervorspannung folgendes Vorgehen empfohlen (Bezeichnungen siehe Bild 48).

\section{Bestimmen der erforderlichen Fliesskapazität der Armierungen}

Die Kräfte in der oberen und unteren Lage der Armierungen betragen

$$
\begin{aligned}
K_{0}^{*} & =\frac{Z^{*}}{2}\left(1-\frac{2 \cdot e}{Z_{p}}\right)+\frac{\bar{M}^{*}}{Z_{p}} \\
K_{u}^{*} & =\frac{Z^{*}}{2}\left(1+\frac{2 \cdot e}{Z_{p}}\right)-\frac{\bar{M}^{*}}{Z_{p}} \\
\text { wobei } & Z^{*}=\frac{Q^{*}}{2 \cdot Z} \cdot \frac{F_{e L}^{F l}}{F_{e L}} \cdot \tan \beta ; \tan \beta=0.5 \text { bis } \underline{0.6 .}
\end{aligned}
$$

1) Die Schnittkräfte unter der rechnerischen Bruchlast sind mit * bezeichnet. 
Die Exzentrizität e der Querzugkraft $Z^{*} \operatorname{darf} z u$

$$
e \leq e_{\max }=\frac{d}{2}\left(1-\frac{\tau^{*}}{\tau_{\max }^{*}}\right) \quad 2
$$

angenommen werden mit

$$
\tau^{*}=\frac{Q^{*}}{2 \cdot d \cdot z} \cdot \frac{F_{e L}^{F l}}{F_{e L}}
$$

und $\quad \tau_{\max }^{*}=$ abere Schubspannungsgrenze (gemäss Norm).

Das Versatzmass der Armierung, bezogen auf den Trägerquerschnitt mit der Querkraft $Q$, ist anzusetzen zu

$$
v \cong 0.3\left(z+2 \cdot b_{\theta}\right)
$$

Die Biegearmierung im Zugflansch ist "hinter" dem Versatzmass zu verankern.

\section{Wahl der vorgespannten und der schlaffen Armierungen}

Der Vorspanngrad ist nach wirtschaftichen und konstruktiven Gesichtspunkten festzulegen. Im allgemeinen ohne Begrenzung nach oben oder nach unten.

Daraus resultieren die Vorspannkraft und der entsprechende Querschnitt $F_{\text {so }}$ der vorgespanten Armierung.

Die Querschnitte der schlaffen Armierung sind so zu bestimmen, dass

$$
\begin{array}{r}
F_{s 0} \cdot \sigma_{s f}+F_{e 0} \cdot \sigma_{B f}=K_{0}^{*} \\
F_{B u} \cdot \sigma_{\theta f}=K_{U}^{*}
\end{array}
$$

erfüllt wird.

In beiden Armierungslagen ist auf jeden Fall eine schlaffe Mindestarmierung von

$$
\mu_{\min }=0.10 \text { bis } 0.15 \%
$$

einzulegen.

\section{Nachweis der Rissesicherung}

Sofern $\tan \beta=0.6$ und $e=0$ angenommen wurde und eher dünne und gut verteilte Armierungsstäbe gewählt wurden, ist auch in nicht oder nur schwach quer vorgespannten Zugplatten ein befriedigendes Rissverhalten zu erwarten.

In anderen Fällen kann ein solches Verhalten angestrebt werden durch

- die Begrenzung des Spannungszuwachses $\Delta \sigma_{S}=\sigma_{\mathrm{g}}$ in den Armierungen, oder

- die Anwendung einer geeigneten Rissformel.

Diese Empfehlungen gelten für Zugflansche symmetrischer I-Querschnitte. Bemessungsregeln für andere Querschnitte können sinngemäss hergeleitet werden.

2) Begründung siehe im Anhang. 
Les dalles minces comprimées ou tendues des profils en Te et des sections évidées sont fréquemment soumises à des sollicitations combinées d'effort tranchant longitudinal et de flexion transversale qui s'ajoutent à l'effort de compression ou de traction longitudinal.

[1] et [2] décrivent les essais sur dalles minces comprimées de sept poutres en beton armé.

Le présent rapport d'essais traite du problème des dalles minces soumises à une traction longitudinale. Jl s'agit de pouvoir créer les bases pour le développement de modèles de dimensionnement simples et en accord avec le comportement réel. En 1977 et 1978, 1'institut de statique et de construction de l'école polytechnique fédérale de Zurich a réalisé six essais sur poutres de profil I.

On s'est servi essentiellement des modèles suivants pour le dimensionnement de l'armature des dalles minces (figure 3 ).

Effort tranchant longitudinal seul

Poutres 21, 22: Modèle formé d'un système de barres triangulé situé dans l'aile de la poutre (figure 2).

Effort tranchant longitudinal avec flexion transversale/et précontrainte transversale

Poutre 23 : Superposition de l'armature résistant à l'effort de traction transversal centré et à la flexion transversale.

Poutre 25 : Superposition des forces résultant de l'effort de traction excentrique et de la flexion transversale.

Poutres 24, 26: Superposition des forces résultant de l'effort de traction excentrique et de la flexion transversale.

La dalle mince des poutres 23 à 26 fut partiellement précontrainte transversalement dans la moitié droite de la portée.

L'armature longitudinale fut répartie symétriquement dans l'aile à l'exception de la poutre 22 . L'armature de cette dernière fut placke à $75 \%$ dans l'aile et à $25 \%$ dans l'âme.

Tous les autres paramètres tels que les dimensions de la pièce, I'armature à l'effort tranchant dans l'âme, l'armature de compression dans l'aile, la qualité du béton, etc. furent maintenance constants (figure 5 ).

Se référant aux résultats des essais et aux deductions présentées au chapitre 5 , nous pouvons recommander le procédé suivant pour le dimensionnement de l'armature des dalles minces tendues subissant un effort tranchant longitudinal avec flexion transversale et avec une précontrainte transversale (notations of figure 48 ). 


\section{Détermination de la capacité portante nécessaire des armatures}

Les forces reprises par la couche superieure et inférieure des armatures sont les suivantes:

où

$$
\begin{aligned}
& K_{0}^{*}=\frac{Z^{*}}{2}\left(1-\frac{2 \cdot e}{Z_{p}}\right)+\frac{\bar{M}^{*}}{Z_{p}} \\
& K_{u}^{*}=\frac{Z^{*}}{2}\left(1+\frac{2 \cdot e}{Z_{P}}\right)-\frac{\bar{M}^{*}}{Z_{p}} \\
& Z^{*}=\frac{Q^{*}}{2 \cdot Z} \cdot \frac{F_{e L}^{F l}}{F_{e L}} \cdot \tan \beta ; \tan \beta=0.5 \div \underline{0.6 .}
\end{aligned}
$$

L'excentricité e de la force de traction transversale peut être évaluée de la manière suivante

où $\quad \tau^{*}=\frac{Q^{*}}{2 \cdot d \cdot z} \cdot \frac{F^{F I}}{F_{e L}}$

$$
E \leq e_{\max }=\frac{d}{2}\left(1-\frac{\tau^{*}}{\tau_{\max }^{*}}\right)
$$

et

$$
\tau_{\max }^{*} \text { : limite supérieure de l'effort tangentiel (selon la norme). }
$$

L'armature transversale est décalée par rapport à la section envisagée soumise à l'effort tranchant $Q$ d'une longueur égale à

$$
v=\cong 0.3\left(z+2 \cdot b_{e}\right)
$$

L'armature de flexion dans l'aile tendue doit ètre ancrée au-delà de cette distance de décalage.

\section{Choix de l'armature précontrainte et de l'armature passive}

Le degré de précontrainte est choisi selon des critères économiques et constructifs. Généralement on ne fixe pas de borne supérieure ni inférieure.

On détermine ainsi la force de précontrainte et la section correspondante de l'armature de précontrainte $F_{\text {so }}$.

Les sections de l'armature passive sont déterminées par les équations suivantes:

$$
\begin{array}{r}
F_{s o} \cdot \sigma_{s f}+F_{e 0} \cdot \sigma_{e f}=K_{0}^{*} \\
F_{e u} \cdot \sigma_{e f}=K_{u}^{*}
\end{array}
$$

Il faut toutefois placer une armature minimale pour chaque couche d'armature

$$
\mu_{\min }=0.10 \div 0.15 \%
$$

1) Les efforts de réduction calculés avec. Ia charge théorique de rupture portent le signe *.

2) Explications of Annexe 


\section{Contrôle de la fissuration dans le domaine d'utilisation}

En admettant des valeurs $\tan \beta=0.6$ et $e=0$ et en choisissant des barres d'armature plutôt fines et bien réparties, on peut s'attendre à un comportement à la fissuration favorable dans les dalles minces tendues avec aucune ou avec une faible armature de précontrainte transversale.

Dans d'autres cas on peut tendre vers un même comportement de fissuration favorable avec:

- la limitation de l'accroissement de contrainte $\Delta \sigma_{5}=\sigma_{e}$ dans l'armature ou bien avec

- l'application d'une formule adéquate permettant d'évaluer la fissuration.

Ces recommandations se rapportent aux ailes tendues des sections symetrique en I. Des règles de dimensionnement pour d'autres sections peuvent être déduites en adoptant un raisonnement conforme. 
Compression and tension flanges of thin-walled $T$ - and hollow box beams are subjected to longitudinal shear and transverse bending in addition to longitudinal compression and tension respectively.

Tests carried out on the compression flanges of seven reinforced concrete beams were reported in references [1] and [2].

The following report describes tests carried out during 1977-78 on the tension flanges of six reinforced concrete I-beams at the Institute of Structural Engineering. Swiss Federal Institute of Technology Zurich. Simple models for the dimensioning of such members are also proposed.

Calculation of the transverse slab reinforcement was based on the following models (Fig. 3):

Longitudinal shear only

Beams $21, \mathrm{Z2}$ : Flange truss model (Fig. 2)

Longitudinal shear with transverse bending and transverse prestressing

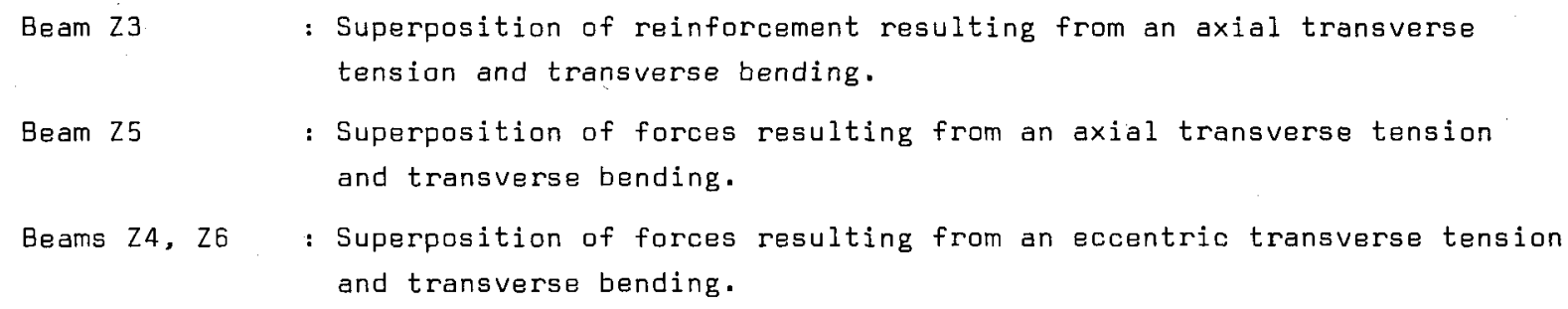

The tension flanges on the right hand half spans of beams $Z 3, Z 4, Z 5$ and Z6 were partially prestressed in the transverse direction.

The longitudinal bending reinforcement was arranged symmetrically in the tension flange. The only exception to this was beam 22 , where $75 \%$ of this reinforcement was placed in the flange and the remaining $25 \%$ distributed in the web.

All other parameters such as beam dimensions, web shear reinforcement, compression flange reinforcement, concrete quality etc. were held constant for all the beams (Fig. 5 ).

Based on the test results and the conclusions set out in Section 5 of this report, the following design rules are suggested for the reinforcement in tension flanges of reinforced concrete beams subjected to longitudinal shear with transverse bending and transverse prestressing (see Fig. 48 for notations and symbols): 


\section{Determination of required yield capacity of reinforcement}

The forces in the upper and lower layers of reinforcement are given by

$$
\begin{aligned}
& K_{0}^{*}=\frac{Z^{*}}{2}\left(1-\frac{2 \cdot e}{Z_{p}}\right)+\frac{\bar{M}^{*}}{z_{p}} \\
& K_{u}^{*}=\frac{Z^{*}}{2}\left(1+\frac{2 e}{z_{p}}\right)-\frac{\bar{M}^{*}}{Z_{p}}
\end{aligned}
$$

where $\quad Z^{*}=\frac{Q^{*}}{2 \cdot Z} \cdot \frac{F^{F L}}{F_{e L}} \cdot \tan \beta ; \tan \beta=0.5$ bis $\underline{0.6}$.

The eccentricity $e$ of the transverse tensile force $Z^{*}$ may be assumed to be given by

$$
\left.B \leq e_{\max }=\frac{d}{2}\left(1-\frac{\tau^{*}}{\tau_{\max }}\right) \quad 2\right)
$$

where

$$
\begin{aligned}
& \tau^{*}=\frac{Q^{*}}{2 \cdot d \cdot z} \cdot \frac{F_{B L}^{F l}}{F_{e L}} \\
& \tau_{\text {max }}=\text { upper limit to shear stress (according to code) }
\end{aligned}
$$

The displacement distance for the reinforcement in a beam cross-section under transverse shear $Q$ is given by

$$
v \cong 0.3\left(z+2 \cdot b_{e}\right)
$$

The bending reinforcement should be anchored beyond the displacement point given by this expression.

\section{Choice of prestressed and non-prestressed reinforcement}

The degree of prestressing is to be chosen with due regard to economic and detailing aspects. As a general rule any value of degree of prestressing may be chosen.

Once this value has been chosen, the value of the prestressing force and the required area of prestressed reinforcement can easily be calculated.

The area of non-prestressed reinforcement is then chosen to satisfy the following conditions

$$
\begin{array}{r}
F_{s 0} \cdot \sigma_{s f}+F_{e 0} \cdot \sigma_{e f}=K_{0}^{*} \\
F_{e u} \cdot \sigma_{e f}=K_{u}^{*}
\end{array}
$$

A minimum reinforcement on each side of $\mu_{\min }=0.10$ to $0.15 \%$ should be provided.

1) Force with the suffix * are ultimate values

2) See appendix for derivation 


\section{Checking crack behaviour}

With the assumptions of $\tan \beta=0.6$ and $e=0$ and the choice of well-distributed smalldiameter reinforcement, a satisfactory crack behaviour may be expected in tension flanges without or only with light prestressing.

In other cases a satisfactory crack behaviour may be achieved through

- limiting the stress increase in the prestressed and non-prestressed reinforcement $\Delta \sigma_{s}$ and $\sigma_{e}$ respectively, caused by cracking

- limiting crack widths calculated by suitable formulae.

These recommendations are valid for the tension of symmetrical I-cross-sections. Design rules for other types of cross-sections may be derived from these recommendations. 
Der vorliegende Versuchsbericht ist im Rahmen der 3. Phase des Forschungsprojektes "Teilweise Vorspannung im Brückenbau" ausgearbeitet worden. Dieses Programm wurde durch einen Beitrag aus dem

Kredit zur Förderung der Strassenbauforschung

des Eidgenössischen Departementes des Innern ermöglicht. Für diese grosszügige finanzielle Unterstützung möchten die Verfasser dem Eidg. Departement des Innern und der Kommission für Strassenbauforschung herzlich danken.

Den Firmen

Monteforno Stahl- und Walzwerke $A G$, Bodio von Moos Stahl AG, Luzern

sind die Verfasser für die zur Verfügung gestellten Armierungsstähle zu Dank verpflichtet. Den Firmen

Cementfabrik Holderbank

Weiacher Kies $A G$

danken wir für die Ueberlassung von Zement und Zuschlagstoffen für die Betonherstellung.

Der Firma Stahlton AG, Zürich

danken wir für ihre Unterstützung bei der Ausführung der Quervorspannung bestens. 
[1] Badawy M., Bachmann H.: "Versuche über Längsschub und Querbiegung in Druckplatten von Betonträgern". Institut für Baustatik und Konstruktion, ETH Zürich, Versuchsbericht Nr. 6504-8, Juni 1977, Birkhäuser Verlag Basel und Stuttgart.

[2] Bacchetta A., Bachmann H.: "Versuche zur teilweisen Vorspannung für Längsschub und Querbiegung in Druckplatten von Betonträgern". Institut für Baustatik und Konstruktion, ETH Zürich, Versuchsbericht Nr. 6504-9, Juli 1977, Birkhäuser Verlag Basel und Stuttgart.

[3] Bachmann H.: "Längsschub und Querbiegung in Druckflanschen von Betonträgern". Institut für Baustatik und Konstruktion, ETH Zürich, Bericht Nr. 79, März 1978 Sonderdruck aus Beton- und Stahlbetonbau Nr. 3, März 1978, Birkhäuser Verlag Basel und Stuttgart.

[4] Bachmann H., Bacchetta A.: "Teilweise Vorspannung für Längsschub und Querbiegung in Druckplatten von Betonträgern". Institut für Baustatik und Konstruktion. ETH Zürich, Bericht Nr. 82, 1978, Sonderdruck aus Beton- und Stahlbetonbau Nr. 5, 1978, Birkhäuser Verlag Basel und Stuttgart.

[5] Norm SIA 162 (1968): "Norm für die Berechnung, Konstruktion und Ausführung von Bauwerken aus Beton, Stahlbeton und Spannbeton. Schweizerischer Ingenieur- und Architekten-Verein (SIA), Zürich.

[6] Thürlimann B.: "Schubbemessung bei Querbiegung", Institut für Baustatik und Konstruktion, ETH Zürich, Bericht Nr. 72, 1977, Sonderdruck aus Schweiz. Bauzeitung. Heft 26, 1977. Birkhäuser Verlag Basel und Stuttgart. 


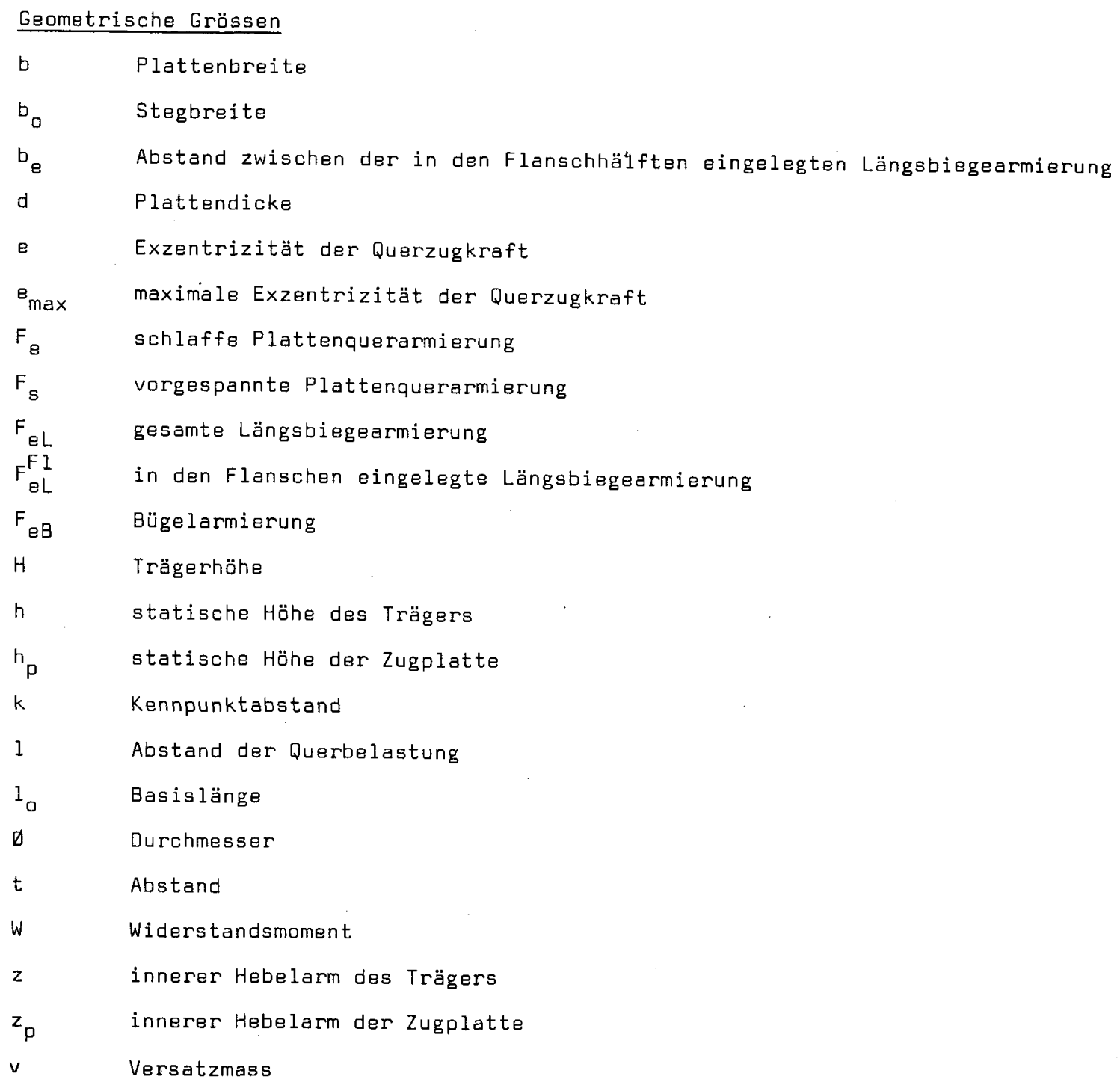

\section{Kraftgrössen}

D Druckkraft, Diagonaldruckkraft

$\triangle \mathrm{D} \quad$ Druckkraft-Zuwachs

$k \quad$ Zugkraft in der Plattenquerarmierung

$K_{0} \quad$ Zugkraft in der oberen Armierungslage

$K_{u} \quad$ Zugkraft in der unteren Armierungslage

M Bisgemoment

$\bar{M} \quad$ Querbiegemoment

$\mathrm{m}_{\mathrm{D}} \quad$ Querbiege-Druckkraft

$m_{z} \quad$ Querbiege-Zugkraft

$P \quad$ Längsbelastung

$P_{f}^{T h} \quad$ theoretische Fliesslast der Plattenquerarmierung infolge von Längsschub

$P$ Th theoretische Biegekapazität der Träger

$\bar{P} \quad$ Querbelastung

$\vec{P}_{f}^{T h} \quad$ theoretische Fliesslast der Plattenquerarmierung infolge von Querbiegung 


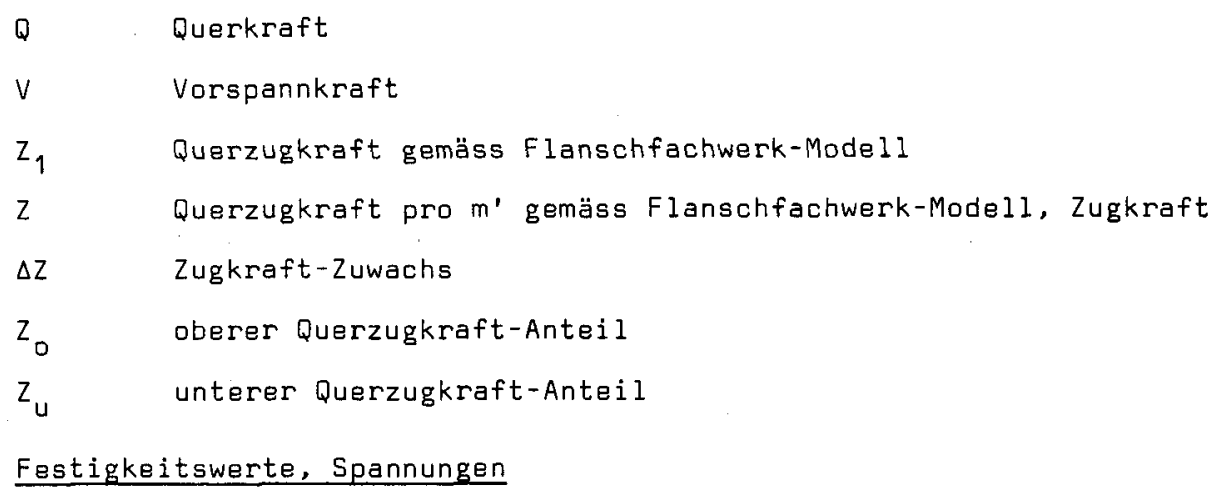

\section{Festigkeitswerte, Spannungen}

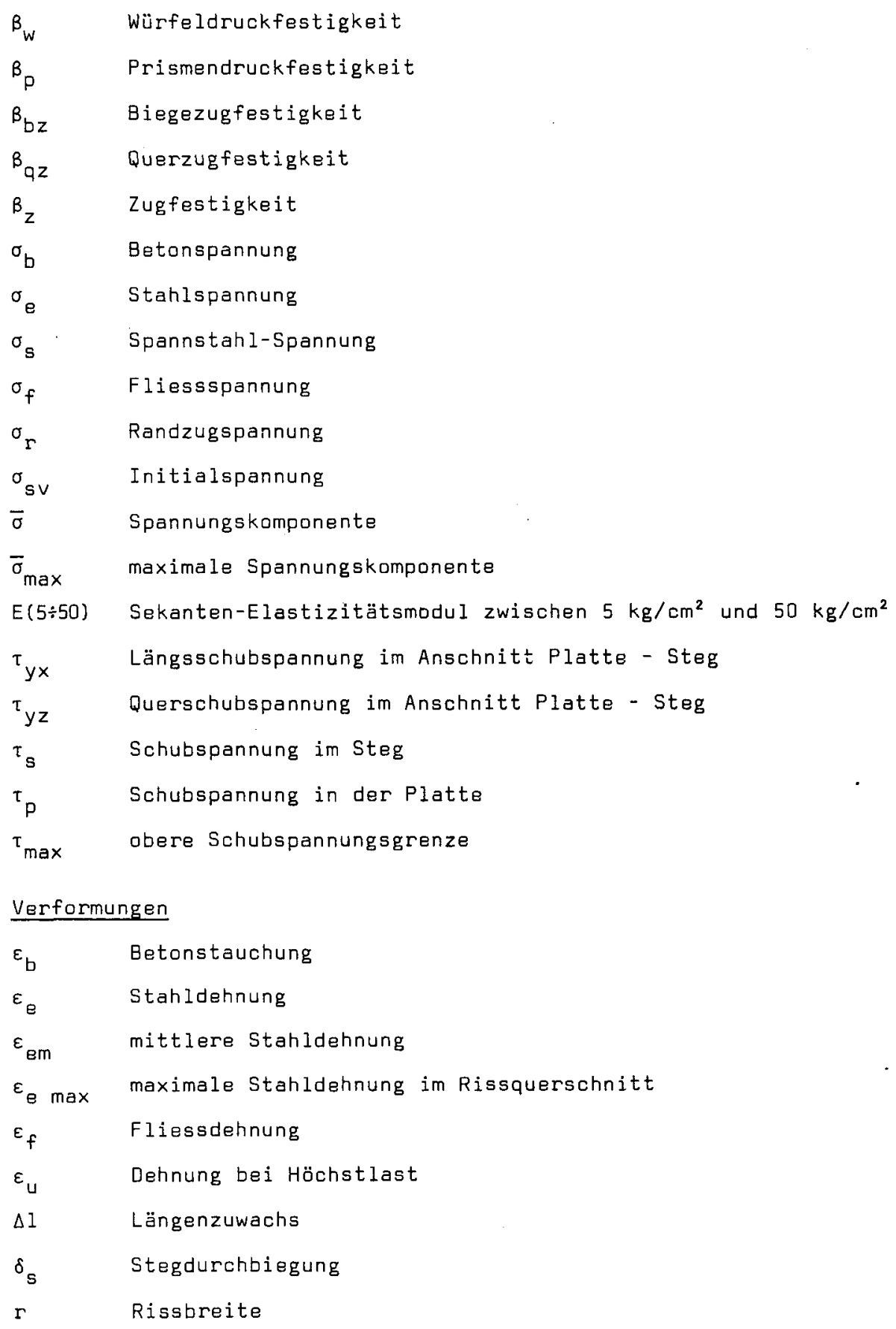




\section{Dimensionslose Parameter}

$\alpha \quad$ Neigung der Betondruckdiagonalen

B Ausbreitwinkel der Betondruckdiagonalen im Zugflansch

$\gamma$ Zugkraft-Momenten-Verhältnis bei kombinierter Beanspruchung

$\lambda \quad$ Fliesskraftverhältnis

K' Vorspanngrad

$k \quad$ Verbundkoeffizient

$\mu \quad$ Armierungsgehalt der Plattenquerarmierung

$\mu_{L} \quad$ Armierungsgehalt der Längsarmierung

$\mu_{B} \quad$ Armierungsgehalt der Bügel im Steg

$\mu_{\min }$ Mindestarmierung

Indizns

I.II,III Stufen der Längs- bzw. Querbelastung

A Anfangslast

B Bügel

E Endlast

Ex experimentell

Fl Flansch

L längs

P Platte

Th theoretisch

b Beton

d dynamisch

e Stahl

f fliessen

o oben

s Spannstahl, statisch

u unten 
Stähle

\begin{tabular}{|c|c|c|c|c|c|c|c|}
\hline $\begin{array}{c}\varnothing \\
\mathrm{mm}\end{array}$ & $\begin{array}{c}F_{e} \\
\mathrm{~cm}^{2}\end{array}$ & $\begin{array}{c}\sigma_{f d} \\
t / \mathrm{cm}^{2}\end{array}$ & $\begin{array}{c}\sigma_{\mathrm{fs}} \\
t / \mathrm{cm}^{2}\end{array}$ & $\begin{array}{c}\beta_{2 d} \\
t / \mathrm{cm}^{2}\end{array}$ & $\begin{array}{c}\beta_{2 s} \\
t / \mathrm{cm}^{2}\end{array}$ & Stahlqualität & Verwendung \\
\hline 4 & 0.125 & 17.90 & 17.20 & 19.70 & 18.80 & Spanndraht & $\begin{array}{l}\text { Querarmierung } \\
\text { Zugplatte Z3-Z6 }\end{array}$ \\
\hline 6 & 0.281 & 4.92 & 4.75 & 5.42 & 5.18 & Tor (behandelt) & $\begin{array}{l}\text { Querarmierung } \\
\text { Zugplatte Z } 1-\mathrm{Z2}\end{array}$ \\
\hline 6 & 0.281 & 5.57 & 5.28 & 6.42 & 6.10 & Tor (behandelt) & $\begin{array}{l}\text { Querarmierung } \\
\text { Zugplatte Z3-Z6 }\end{array}$ \\
\hline 8 & 0.503 & 5.55 & 5.25 & 7.14 & 6.68 & Box-Ultra & $\begin{array}{l}\text { Querarmierung } \\
\text { Zugplatte Z3-Z6 }\end{array}$ \\
\hline 12 & 1.08 & 5.19 & 4.95 & 7.00 & 6.66 & Box-Ulitra & $\begin{array}{l}\text { Stegarmierung } \\
\mathrm{Z1}-\mathrm{Z} 6\end{array}$ \\
\hline 26 & 5.31 & 5.20 & 4.94 & 6.60 & 6.17 & Box-Ultra & $\begin{array}{l}\text { Leingsarmierung } \\
\mathrm{Z1}-\mathrm{Z6}\end{array}$ \\
\hline
\end{tabular}

Tabelle 1: Eigenschaften der Stähle (Mittelwerte)

Beton

\begin{tabular}{|ll|}
\hline Sand $0-4 \mathrm{~mm}$ & $640 \mathrm{~kg} / \mathrm{m}^{3}$ \\
Sand $4-8 \mathrm{~mm}$ & $560 \mathrm{~kg} / \mathrm{m}^{3}$ \\
Kies $8-16 \mathrm{~mm}$ & $680 \mathrm{~kg} / \mathrm{m}^{3}$ \\
Zement & $325 \mathrm{~kg} / \mathrm{m}^{3}$ \\
Wasser & $180 \mathrm{~kg} / \mathrm{m}^{3}$ \\
W/Z Faktor & 0.55 \\
Raumgewicht & $2385 \mathrm{~kg} / \mathrm{m}^{3}$ \\
Konsistenz & plastisch \\
\hline
\end{tabular}

Tabelle 2 : Zusammensetzung des Betons

\section{Zementmörtel}

\begin{tabular}{|ll|}
\hline Sand $0-4 \mathrm{~mm}$ & $1480 \mathrm{~kg} / \mathrm{m}^{3}$ \\
Zement & $420 \mathrm{~kg} / \mathrm{m}^{3}$ \\
Wasser & $252 \mathrm{~kg} / \mathrm{m}^{3}$ \\
$W / Z$ Faktor & 0.60 \\
Raumgewicht & $2160 \mathrm{~kg} / \mathrm{m}^{3}$ \\
Konsistenz & erdfeucht \\
\hline
\end{tabular}

Tabelle 5: Zusammensetzung

\section{des Zementmörtels}

Beton

\begin{tabular}{|c|c|c|c|}
\hline$\beta_{w}\left(\mathrm{~kg} / \mathrm{cm}^{2}\right)$ & $\beta_{p}\left(\mathrm{~kg} / \mathrm{cm}^{2}\right)$ & $\beta_{\mathrm{bz}}\left(\mathrm{kg} / \mathrm{cm}^{2}\right)$ & $\beta_{\mathrm{qz}}\left(\mathrm{kg} / \mathrm{cm}^{2}\right)$ \\
\hline 375 & 320 & 53 & 23 \\
\hline
\end{tabular}

Tabelle 3: Konventionelle Festigkeitsweite *)

\begin{tabular}{|c|c|c|c|}
\hline$\beta_{p}^{\sigma-\varepsilon}\left(\mathrm{kg} / \mathrm{cm}^{2}\right)$ & $\varepsilon_{u}(\% 0)$ & $E_{3-50}\left(t / \mathrm{cm}^{2}\right)$ & $E_{5+0 \sigma^{j}}\left(t / \mathrm{cm}^{2}\right)$ \\
\hline 305 & 19 & 370 & 360 \\
\hline
\end{tabular}

Tabelle 4: Spannungs-Dehnungsverhalten der Betonprismen *)

Zementmörtel

\begin{tabular}{|c|c|c|}
\hline$\beta_{w}\left(\mathrm{~kg} / \mathrm{cm}^{2}\right)$ & $\beta_{p}\left(\mathrm{~kg} / \mathrm{cm}^{2}\right)$ & $\beta_{b z}\left(\mathrm{~kg} / \mathrm{cm}^{2}\right)$ \\
\hline 290 & 242 & 50 \\
\hline
\end{tabular}

Tabelle 6: Konventionelle Festigkeitswerte *)

*) Mittelwerte, im Prüfalter von 28 Togen 
Vorspanngrad : $\kappa^{\prime}=\frac{M_{\text {zul.V }}}{M_{g+p}}$

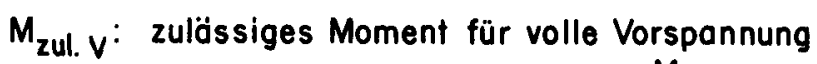
$M_{g+p}$ : Moment unter Gebrauchslast $\left(\frac{M_{p l}}{1.8}\right)$

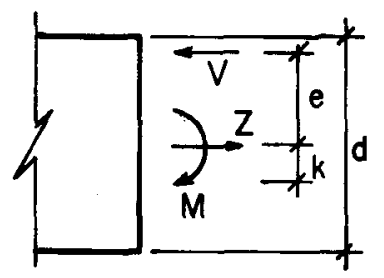

Querschnittswerte :

$d, e, F, k, w$

Querschnittskräfte:

$Z, M, V, \gamma=\frac{Z}{M / d}$ (Zugkraft-Momenten-Verhältnis)

Untere Betonrandspannung am ungerissenen Querschnitt:

$$
\begin{aligned}
& \sigma_{r}=\frac{M_{z u l . V}}{W}+\frac{z}{F}-\frac{V \cdot e}{W}-\frac{V}{F} \\
& \sigma_{r}=M_{z u l . V} \cdot \frac{1+y \frac{k}{d}}{W}-V \cdot \frac{e+k}{W}
\end{aligned}
$$

Zulässiges Moment für volle Vorspannung :

$$
M_{\text {zul.V }}=\frac{\sigma_{r} \cdot W+V(e+k)}{1+\gamma \frac{k}{d}}
$$

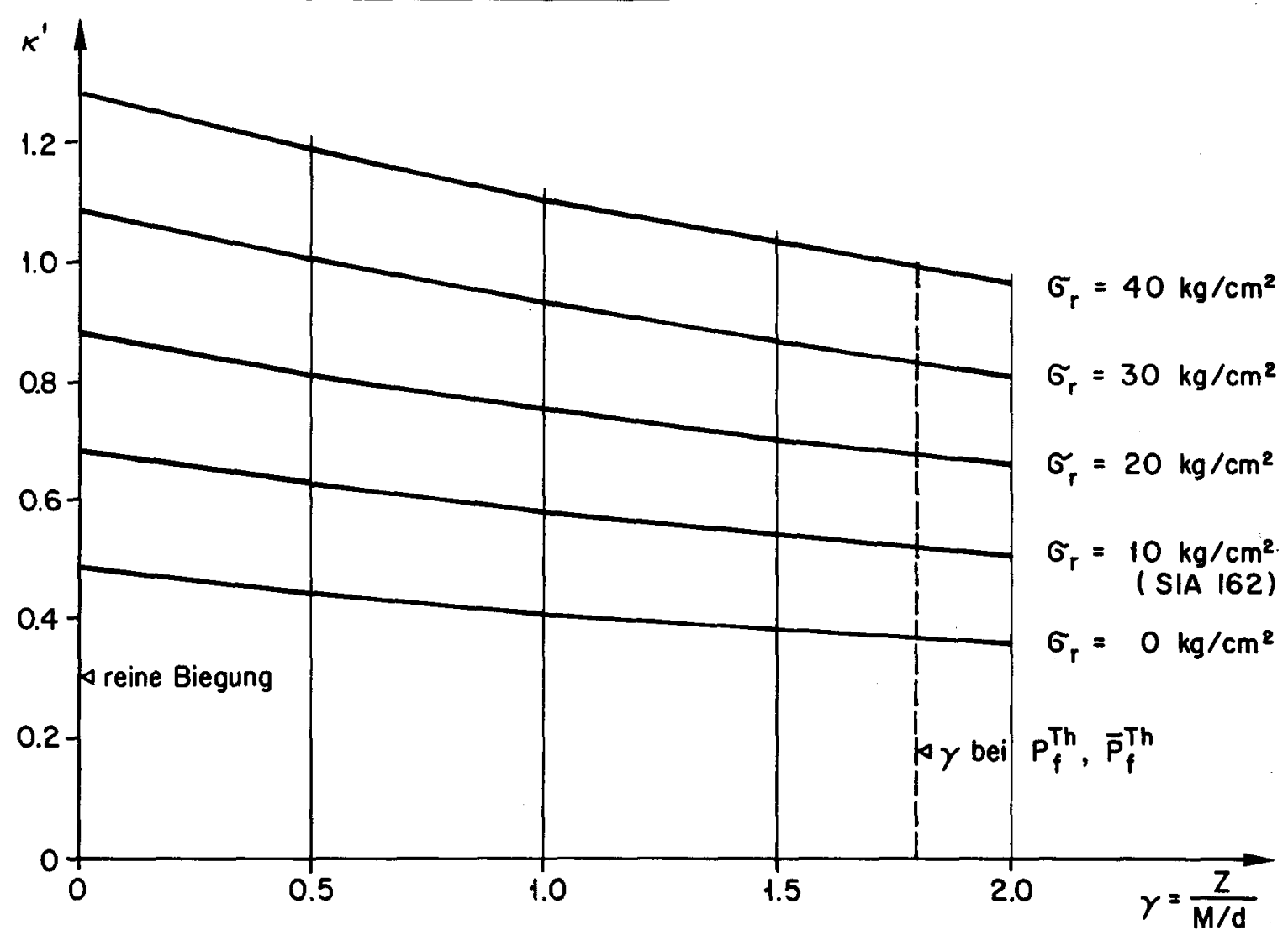

Tabelle 7: Einfluss von $\sigma_{r}$ und $\gamma$ auf den Vorspanngrad $K^{\prime}$ im Anschnitt Zugplatte/Steg der Träger Z3, Z 5 und Z6 (ungerissener Querschnitt, Biegung mit zentrischem Zug ) 


\begin{tabular}{|c|c|c|c|c|c|c|c|c|c|c|}
\hline \multirow{3}{*}{ Träger } & \multicolumn{6}{|c|}{ Theoretische Werte } & \multirow{2}{*}{\multicolumn{4}{|c|}{$\frac{\text { Experimentelle Werte }}{\text { Zugplatte }}$}} \\
\hline & \multicolumn{2}{|c|}{ Steg } & \multicolumn{4}{|c|}{ Zugplatte } & & & & \\
\hline & $\begin{array}{l}P_{\text {Th }}^{\text {Th }} \\
\text { (t) }\end{array}$ & $\begin{array}{c}\tau_{\mathrm{s}} \\
\left(\mathrm{kg} / \mathrm{cm}^{2}\right)\end{array}$ & $\begin{array}{c}P_{f}^{T h} \\
(t)\end{array}$ & $\left|\begin{array}{c}\tau_{\mathrm{yx}} \\
\left(\mathrm{kg} / \mathrm{cm}^{2}\right)\end{array}\right|$ & $\begin{array}{l}\bar{P}_{f}^{T h} \\
(1)\end{array}$ & $\begin{array}{c}\tau_{\mathrm{yz}} \\
\left(\mathrm{kg} / \mathrm{cm}^{2}\right)\end{array}$ & $\begin{array}{l}P_{u}^{E x} \\
(t)\end{array}$ & $\begin{array}{l}P_{f}^{E x} \\
(t)\end{array}$ & $\begin{array}{l}\bar{P}_{f} E_{f} \\
(t)\end{array}$ & $\begin{array}{c}\tau_{y x} \\
\left(\mathrm{~kg} / \mathrm{cm}^{2}\right)\end{array}$ \\
\hline 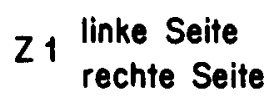 & 58 & 58 & 43 & 43 & - & - & $\begin{array}{c}\text { Bruch } \\
52\end{array}$ & $\begin{array}{l}40 \\
43\end{array}$ & - & 52 \\
\hline $22 \begin{array}{l}\text { linke Seite } \\
\text { rechte Seite }\end{array}$ & 58 & 58 & $\begin{array}{l}56 \\
43\end{array}$ & $\begin{array}{l}42 \\
32\end{array}$ & - & - & $58^{1)}$ & $\begin{array}{l}49 \\
46\end{array}$ & - & 43 \\
\hline Z3 linke Seite & 58 & 58 & 43 & 43 & 3.9 & 5.4 & $\begin{array}{c}\text { Bruch } \\
58\end{array}$ & $\begin{array}{l}43 \\
46\end{array}$ & 3.9 & 58 \\
\hline Z4 $\begin{array}{l}\text { linke Seite } \\
\text { rechte Seite }\end{array}$ & 58 & 58 & 43 & 43 & 6.0 & 8.3 & 43 & 43 & $\begin{array}{c}\text { Bruch } \\
6.0\end{array}$ & 43 \\
\hline 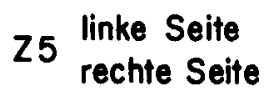 & 58 & 58 & 43 & 43 & 3.9 & 5.4 & $\begin{array}{c}58 \\
\text { Bruch }\end{array}$ & $\begin{array}{l}43 \\
46\end{array}$ & 3.9 & 58 \\
\hline Z6 $\begin{array}{l}\text { linke Seite } \\
\text { rechte Seite }\end{array}$ & 58 & 58 & 43 & 43 & 3.9 & 5.4 & $\begin{array}{c}\text { Bruch } \\
56\end{array}$ & $\begin{array}{r}33 \\
>33\end{array}$ & 3.9 & 56 \\
\hline
\end{tabular}

1) Biegefliessen der Löngsarmierung

Tabelle 8: Theoretische und experimentelle Werte

\begin{tabular}{|c|c|c|}
\hline \multicolumn{3}{|c|}{ Träger } \\
\hline LS & $P_{A}(t)$ & $P_{E}(t)$ \\
\hline 1 & 2.5 & 2.5 \\
2 & 12.0 & 11.8 \\
3 & 18.0 & 17.5 \\
4 & 24.0 & 23.5 \\
5 & 33.0 & 31.8 \\
6 & 40.0 & 38.7 \\
7 & 43.0 & 41.7 \\
8 & 46.0 & 44.3 \\
9 & 49.0 & 46.6 \\
10 & 52.0 & Bruch \\
& & \\
& & \\
& & \\
\hline
\end{tabular}

Tabelle 9 : Belastungsprotokoll Träger Z 1

\begin{tabular}{|r|c|c|}
\hline \multicolumn{3}{|c|}{ Träger } \\
\hline LS & $P_{A}(t)$ & $P_{E}(t)$ \\
\hline 1 & 2.5 & 2.5 \\
2 & 12.0 & 11.8 \\
3 & 18.0 & 17.7 \\
4 & 24.0 & 23.5 \\
5 & 33.0 & 31.6 \\
6 & 40.0 & 38.1 \\
7 & 43.0 & 41.2 \\
8 & 46.0 & 44.4 \\
9 & 49.0 & 46.9 \\
10 & 52.0 & 49.9 \\
11 & 54.0 & 51.8 \\
12 & 56.0 & 53.9 \\
13 & 58.0 & 55.1 \\
14 & 58.0 & 55.0 \\
\hline
\end{tabular}

Tabelle 10: Belastungsprotokoll Träger Z 2 


\begin{tabular}{|r|c|c|c|c|}
\hline \multicolumn{5}{|c|}{ Träger $Z 3$} \\
\hline LS & $P_{A}(t)$ & $P_{E}(t)$ & $\bar{P}_{A}(t)$ & $\bar{P}_{E}(t)$ \\
\hline 1 & 2.5 & 2.5 & 0.15 & 0.15 \\
2 & 2.5 & 2.5 & 2.15 & 2.10 \\
3 & 24.0 & 23.0 & 2.15 & 2.10 \\
4 & 24.0 & 23.2 & 3.00 & 2.85 \\
5 & 33.0 & 31.7 & 3.00 & 2.90 \\
6 & 33.0 & 32.0 & 3.90 & 3.70 \\
7 & 40.0 & 38.5 & 3.90 & 3.75 \\
8 & 43.0 & 42.2 & 3.90 & 3.75 \\
9 & 46.0 & 44.8 & 3.90 & 3.75 \\
10 & 50.0 & 48.7 & 3.90 & 3.70 \\
11 & 54.0 & 52.5 & 3.90 & 3.70 \\
12 & 58.0 & Bruch & 3.90 & Bruch \\
\hline
\end{tabular}

Tabelle 11 : Belastungsprotokoll Träger 23

\begin{tabular}{|r|c|c|c|c|}
\hline \multicolumn{5}{|c|}{ Träger $Z 4$} \\
\hline LS & $P_{A}(t)$ & $P_{E}(t)$ & $\bar{P}_{A}(t)$ & $\bar{P}_{E}(t)$ \\
\hline 1 & 2.5 & 2.5 & 0.15 & 0.15 \\
2 & 12.0 & 11.7 & 0.15 & 0.15 \\
3 & 24.0 & 23.0 & 0.15 & 0.15 \\
4 & 33.0 & 31.7 & 0.15 & 0.15 \\
5 & 40.0 & 38.2 & 0.15 & 0.15 \\
6 & 43.0 & 41.4 & 0.15 & 0.15 \\
7 & 43.0 & 40.1 & 0.15 & 0.15 \\
8 & 43.0 & 41.3 & 3.30 & 3.20 \\
9 & 43.0 & 41.6 & 4.60 & 4.30 \\
10 & 43.0 & Bruch & 6.00 & Bruch \\
& & & & \\
& & & & \\
\hline
\end{tabular}

Tabelle 12 : Belastungsprotokoll Träger Z 4

\begin{tabular}{|r|c|c|c|c|}
\hline \multicolumn{5}{|c|}{ Träger $Z 5$} \\
\hline LS & $P_{A}(t)$ & $P_{E}(t)$ & $\bar{P}_{A}(t)$ & $\bar{P}_{E}(t)$ \\
\hline 1 & 2.5 & 2.5 & 0.15 & 0.15 \\
2 & 2.5 & 2.5 & 2.15 & 2.05 \\
3 & 24.0 & 23.0 & 2.15 & 2.10 \\
4 & 24.0 & 23.4 & 3.00 & 2.80 \\
5 & 33.0 & 31.6 & 3.00 & 2.90 \\
6 & 33.0 & 32.2 & 3.90 & 3.65 \\
7 & 40.0 & 38.7 & 3.90 & 3.75 \\
8 & 43.0 & 42.1 & 3.90 & 3.75 \\
9 & 46.0 & 45.0 & 3.90 & 3.75 \\
10 & 49.0 & 48.1 & 3.90 & 3.70 \\
11 & 52.0 & 50.7 & 3.90 & 3.70 \\
12 & 54.0 & 53.0 & 3.90 & 3.65 \\
13 & 56.0 & 54.0 & 3.90 & 3.60 \\
14 & 58.0 & Bruch & 390 & Bruch \\
\hline
\end{tabular}

Tabelle 13: Belastungsprotokoll Träger Z 5

\begin{tabular}{|r|c|c|c|c|}
\hline \multicolumn{5}{|c|}{ Träger $Z 6$} \\
\hline LS & $P_{A}(t)$ & $P_{E}(t)$ & $\bar{P}_{A}(t)$ & $\bar{P}_{E}(t)$ \\
\hline 1 & 2.5 & 2.5 & 0.15 & 0.15 \\
2 & 2.5 & 2.5 & 2.15 & 2.05 \\
3 & 24.0 & 23.0 & 2.20 & 2.10 \\
4 & 24.0 & 23.5 & 3.00 & 2.75 \\
5 & 33.0 & 32.0 & 3.00 & 2.80 \\
6 & 33.0 & 32.2 & 3.90 & 3.45 \\
7 & 40.0 & 39.0 & 3.90 & 3.60 \\
8 & 43.0 & 41.7 & 3.90 & 3.60 \\
9 & 46.0 & 44.5 & 3.90 & 3.60 \\
10 & 49.0 & 47.2 & 3.90 & 3.60 \\
11 & 52.0 & 50.0 & 3.90 & 3.60 \\
12 & 54.0 & 50.8 & 3.90 & 3.60 \\
13 & 56.0 & Bruch & 3.90 & Bruch \\
& & & & \\
\hline
\end{tabular}

Tabelle 14: Belastungsprotokoll Träger Z 6 


\begin{tabular}{|c|l|c|c|c|c|c|c|c|c|c|c|}
\cline { 2 - 12 } \multicolumn{2}{c|}{} & \multicolumn{4}{c|}{ linke Seite: $B_{1}-B_{I}$} & \multicolumn{5}{c|}{ rechte Seite : $B_{r}-B_{r}$} \\
\cline { 2 - 12 } & $P_{I}$ & $P_{\text {II }}$ & $P_{\text {III }}$ & $P=49 t$ & $P=54 t$ & $P_{I}$ & $P_{\text {II }}$ & $P_{\text {III }}$ & $P=49 t$ & $P=54 t$ \\
\hline \multirow{2}{*}{$Z 1$} & $K(t)$ & & & & & & 9.4 & 15.5 & 20.5 & $28.11)$ & - \\
\cline { 2 - 12 } & $\tan \beta$ & & & & & & 0.39 & 0.47 & 0.48 & 0.57 & - \\
\hline \multirow{2}{*}{$Z 2$} & $K(t)$ & 6.1 & 9.9 & 15.9 & 21.2 & 26.3 & 4.3 & 8.1 & 17.1 & 20.6 & 21.3 \\
\cline { 2 - 12 } & $\tan \beta$ & 0.34 & 0.40 & 0.50 & 0.57 & 0.65 & 0.24 & 0.33 & 0.53 & 0.56 & 0.53 \\
\hline
\end{tabular}

1) interpolierte Werte

\begin{tabular}{|c|c|c|c|c|c|c|c|c|c|c|c|c|c|}
\hline & \multicolumn{6}{|c|}{$\begin{array}{l}\text { linke Seite: } B_{1}-B_{\mid} \\
\text {(schlaff armiert) }\end{array}$} & \multicolumn{6}{|c|}{$\begin{array}{l}\text { rechte Seite: } B_{r}-B_{r} \\
\text { (teilweise vorgespannt) }\end{array}$} \\
\hline & & $P_{I}$ & $P_{\text {II }}$ & $P_{\text {III }}, \bar{P}_{0}$ & $P_{\text {III }}, \bar{P}_{\text {I }}$ & $P_{\text {III }}, \bar{P}_{\text {II }}$ & $P_{\mathbb{I I}^{\prime}} \bar{P}_{\text {II }}^{3}$ & $P_{I}$ & $P_{\text {II }}$ & $P_{\text {III }}, \bar{P}_{0}$ & $P_{\text {III }}, \bar{P}_{\text {I }}$ & $P_{\text {III }} \bar{P}_{\text {II }}$ & $P_{\text {III }}, \bar{P}_{\text {III) }}^{3)}$ \\
\hline & $\tau\left(\mathrm{kg} / \mathrm{cm}^{2}\right.$ & 24 & 33 & 43 & 43 & 43 & 43 & 24 & 33 & 43 & 43 & 43 & 43 \\
\hline & $e_{\operatorname{mox}}^{2)}(\mathrm{cm})$ & 3.2 & 2.5 & 1.75 & 1.75 & 1.75 & 1.75 & 3.2 & 2.5 & 1.75 & 1.75 & 1.75 & 1.75 \\
\hline \multirow{3}{*}{24} & $z(t)$ & 6.5 & 13.7 & 18.3 & 20.0 & 24.2 & 25.7 & 14.0 & 17.3 & 22.7 & 22.0 & 22.7 & 25.3 \\
\hline & $\tan \beta$ & 0.27 & 0.42 & 0.43 & 0.47 & 0.56 & 0.60 & 0.58 & 0.52 & 0.53 & 0.51 & 0.53 & 0.59 \\
\hline & $e / e_{\max }$ & -0.77 & -0.94 & -1.16 & 0.26 & 0.43 & 1.06 & -1.02 & -1.13 & -1.09 & 0.06 & 0.40 & 1.25 \\
\hline
\end{tabular}

2) theoretischer Ausotz noch GI. (23)

${ }^{3}$ ) extropolierte Werte

\begin{tabular}{|c|c|c|c|c|c|c|c|c|c|}
\hline & \multicolumn{4}{|c|}{$\begin{array}{l}\text { linke Seite: } B_{1}-B_{1} \\
\text { (schlaff armiert) }\end{array}$} & \multicolumn{4}{|c|}{$\begin{array}{l}\text { rechte Seite: } B_{r}-B_{r} \\
\text { (teilweise vorgespannt) }\end{array}$} \\
\hline & & $P_{I}, \bar{P}_{I}$ & $\mathrm{P}_{\mathbf{I}}, \overline{\mathrm{P}}_{\mathrm{II}}$ & $P_{\text {III }}, \bar{P}_{\text {III }}$ & $P=54 t, \bar{P}_{\text {III }}$ & $P_{I}, \bar{P}_{I}$ & $\mathrm{P}_{\mathbf{I I}}, \overline{\mathrm{P}}_{\mathbf{I I}}$ & $P_{\text {III }}, \bar{P}_{\text {III }}$ & $P=54 t, \bar{P}_{\text {III }}$ \\
\hline & $\tau\left(\mathrm{kg} / \mathrm{cm}^{2}\right)$ & 24 & 33 & 43 & 54 & 24 & 33 & 43 & 54 \\
\hline & $\mathrm{e}_{\max }^{2)}(\mathrm{cm})$ & 3.2 & 2.5 & 1.75 & 0.9 & 3.2 & 2.5 & 1.75 & 0.9 \\
\hline \multirow{3}{*}{23} & $Z(t)$ & 9.0 & 1.56 & 26.0 & 34.5 & 7.8 & 15.5 & 26.0 & 34.3 \\
\hline & $\tan \beta$ & 0.38 & 0.47 & 0.60 & 0.65 & 0.33 & 0.47 & 0.60 & 0.64 \\
\hline & $\mathrm{e} / \mathrm{e}_{\max }$ & 0.84 & 0.40 & 0.30 & 0.23 & 0.66 & 0.45 & 0.51 & 0.31 \\
\hline \multirow{3}{*}{ Z 5} & $Z(t)$ & 7.3 & 1.56 & 23.3 & 33.0 & 8.0 & 13.7 & 23.3 & 33.0 \\
\hline & $\tan \beta$ & 0.30 & 0.47 & 0.54 & 0.61 & 0.33 & 0.42 & 0.54 & 0.61 \\
\hline & $\mathrm{e} / \mathrm{e}_{\max }$ & 1.00 & 0.33 & 0.19 & -0.16 & 0.73 & 0.48 & 0.12 & -0.20 \\
\hline \multirow{3}{*}{26} & $Z(t)$ & 8.2 & 1.67 & 24.3 & 2.60 & 9.3 & 15.5 & 22.7 & 26.0 \\
\hline & $\tan \beta$ & 0.34 & 0.51 & 0.56 & 0.48 & 0.39 & 0.47 & 0.53 & 0.48 \\
\hline & $e / e_{\max }$ & 0.70 & 0.23 & 0.37 & 0.92 & 0.64 & 0.28 & 0.29 & 0.89 \\
\hline
\end{tabular}

Tabelle 15: Versuchsresultate in den Messstrecken $a-a$ 

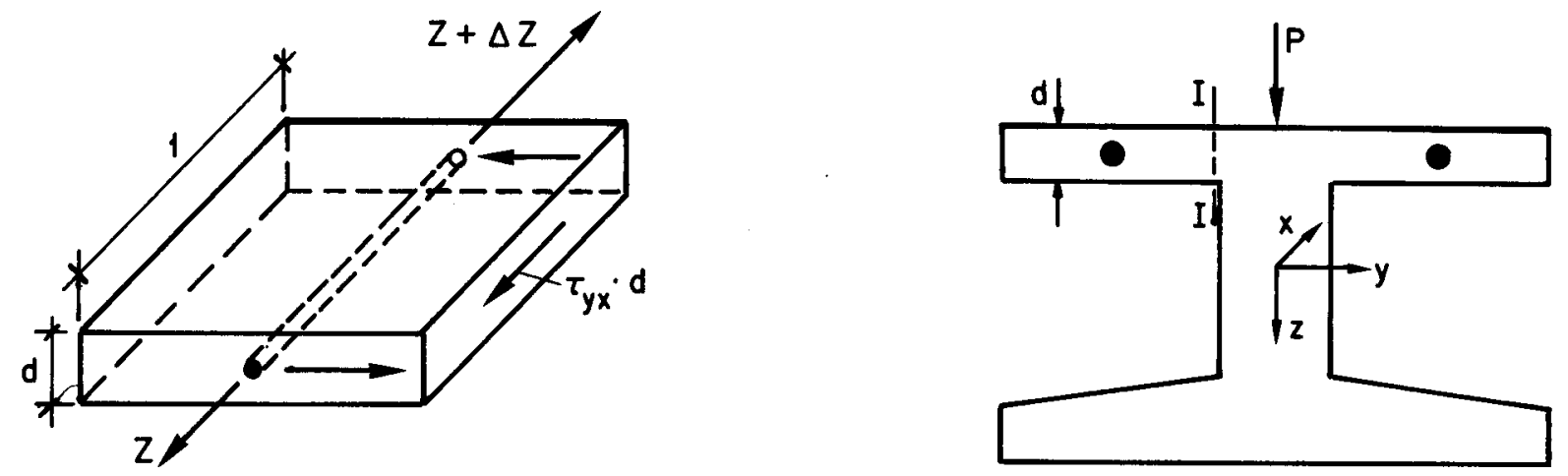

b - Längsschub + Querbiegung
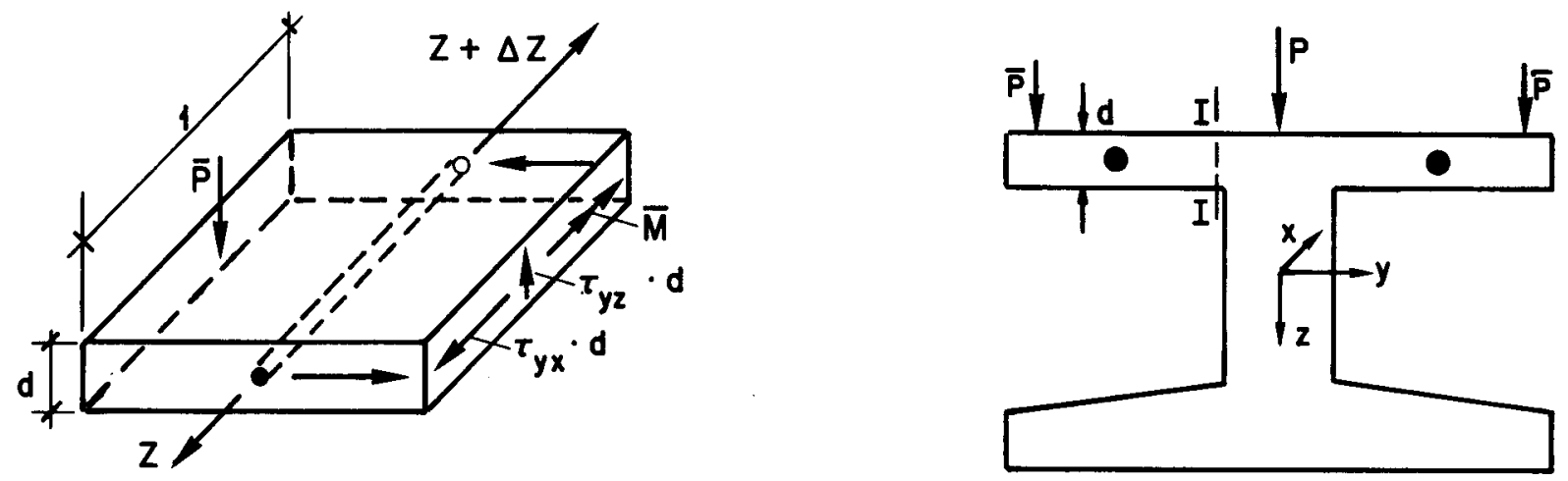

c-Längsschub + Querbiegung + Quervorspannung
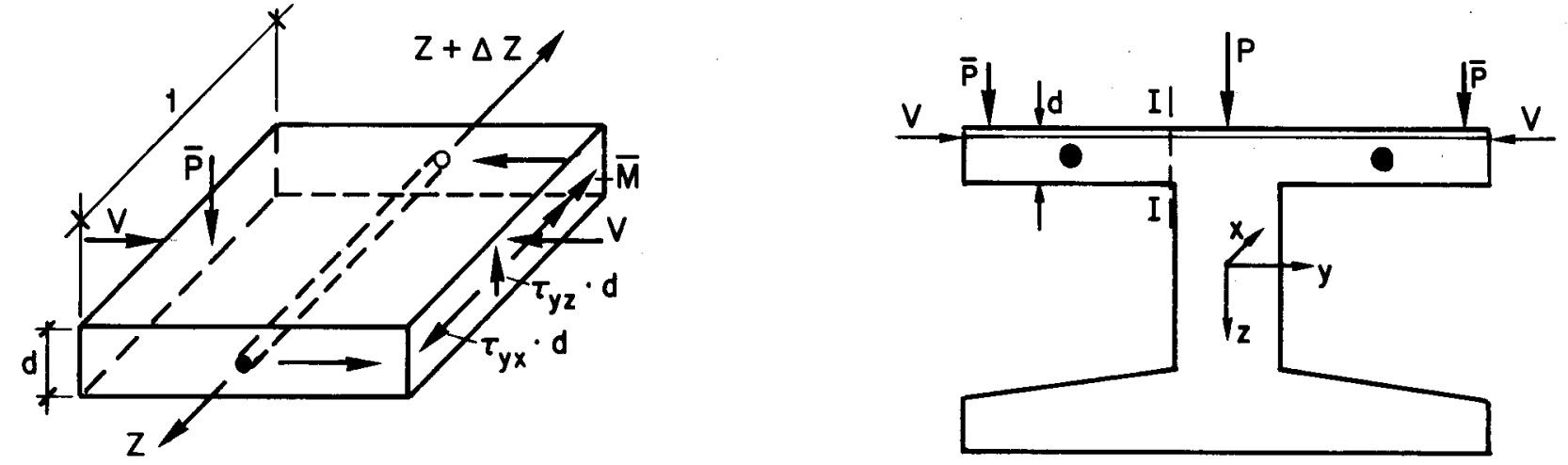

Bild 1: Problemstellung 
Querschnitt :

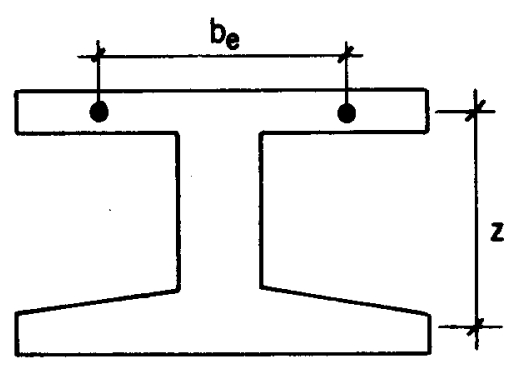

System :

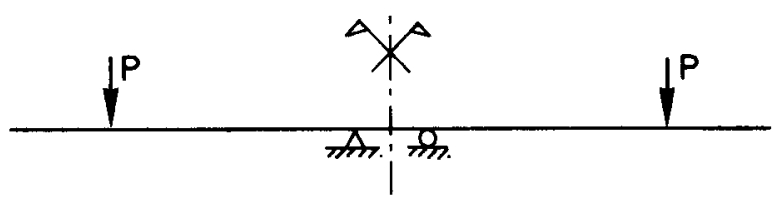

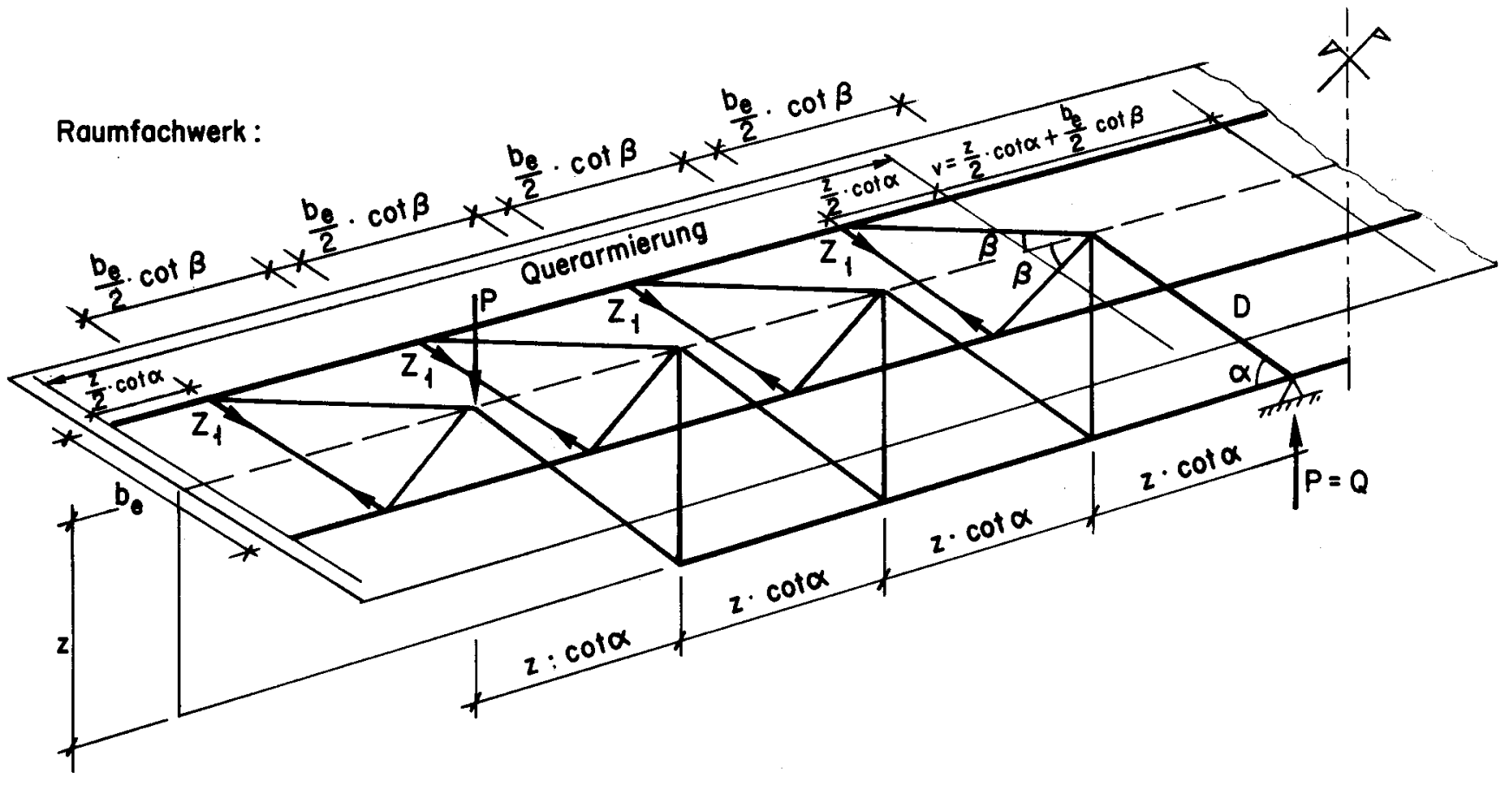

Kräftedreieck:

$D \cdot \cos \alpha=Q \cdot \cot \alpha$

$D^{\prime}=\frac{D}{2} \cdot \cos \alpha=\frac{Q}{2} \cdot \cot \alpha$

$Z_{1}=\frac{Q}{2} \cdot \cot \alpha \cdot \tan \beta$

$Z_{\text {pro } m^{\prime}}=\frac{Q}{2 \cdot z} \cdot \tan \beta$

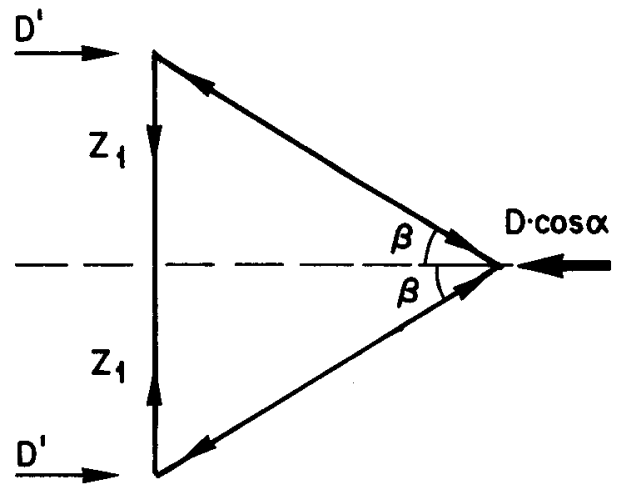

$v=\frac{z}{2} \cdot \cot \alpha+\frac{b_{e}}{2} \cdot \cot \beta$

Bild 2-a: Flanschfachwerk-Modell mi† 2 Zugstäben (ganze Längsbiegearmierung ausserhalb Steg) 
Querschnitt :

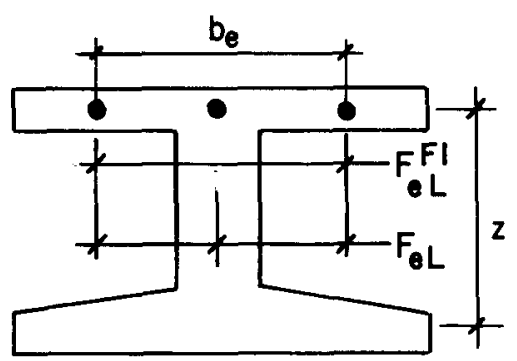

System :

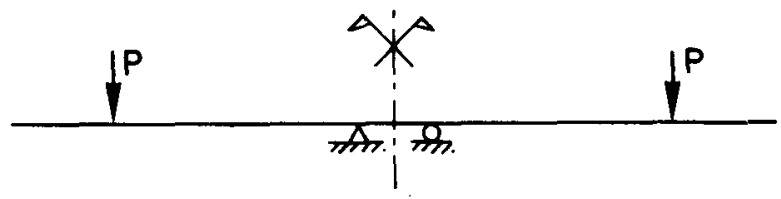

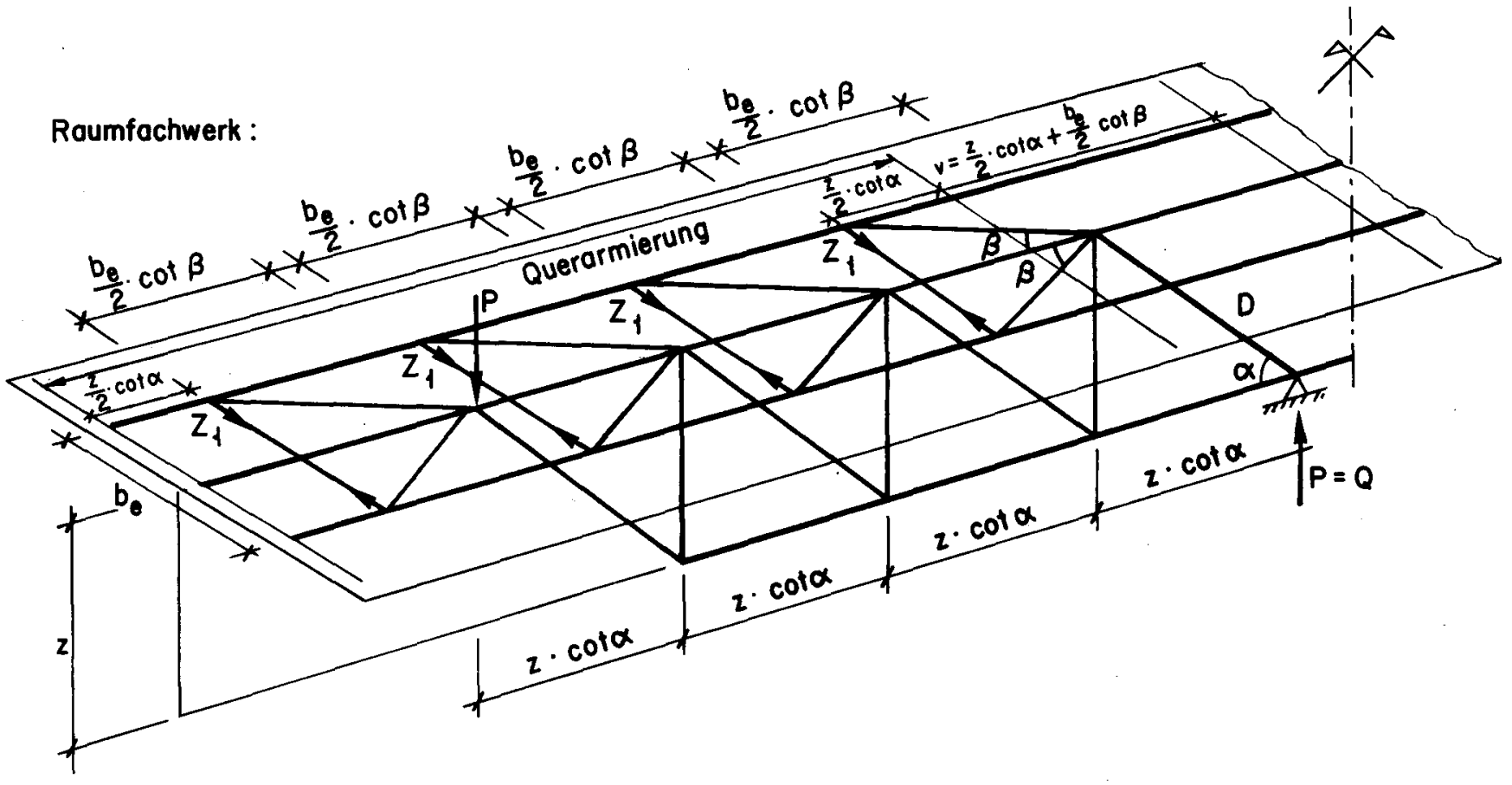

Kräftedreieck :

$D \cdot \cos \alpha \cdot \frac{F_{Q L}^{F I}}{F_{Q L}}=Q \cdot \cot \alpha \cdot \frac{F_{Q L}^{F I}}{F_{Q L}}$

$D^{\prime}=\frac{D}{2} \cdot \cos \alpha \cdot \frac{F_{\mathrm{eL}}^{F I}}{F_{\mathrm{eL}}}=\frac{Q}{2} \cdot \cot \alpha \cdot \frac{F_{\mathrm{eL}}^{\mathrm{FI}}}{F_{\mathrm{eL}}}$

$Z_{1}=\frac{Q}{2} \cdot \cot \alpha \cdot \frac{F_{Q L}^{F I}}{F_{Q L}} \cdot \tan \beta$

$Z_{\text {prom }}=\frac{Q}{2 \cdot z} \cdot \frac{F_{Q L}^{F !}}{F_{\theta L}} \cdot \tan \beta$

$v=\frac{z}{2} \cdot \cot \alpha+\frac{b_{e}}{2} \cdot \cot \beta$

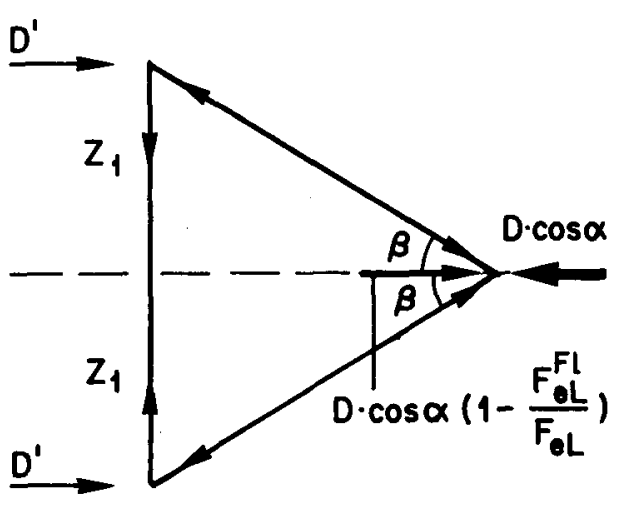

Bild 2-b: Flanschfachwerk-Modell mit 3 Zugstäben (Längsbiegearmierung nur teilweise ausserhalb Steg) 
Armierung für Löngsschub allein; $Z=\frac{Q}{2 \cdot z} \cdot \frac{F_{Q L}^{F l}}{F_{Q L}} \cdot \tan \beta$
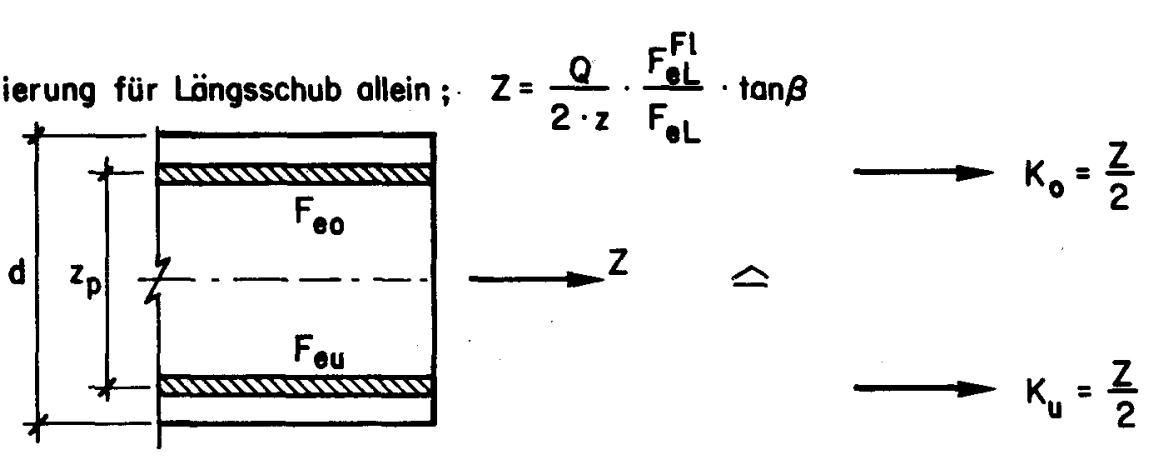

Überlagerung der Armierungen aus zentrisch angreifender Querzugkraft und ous Querbiegung
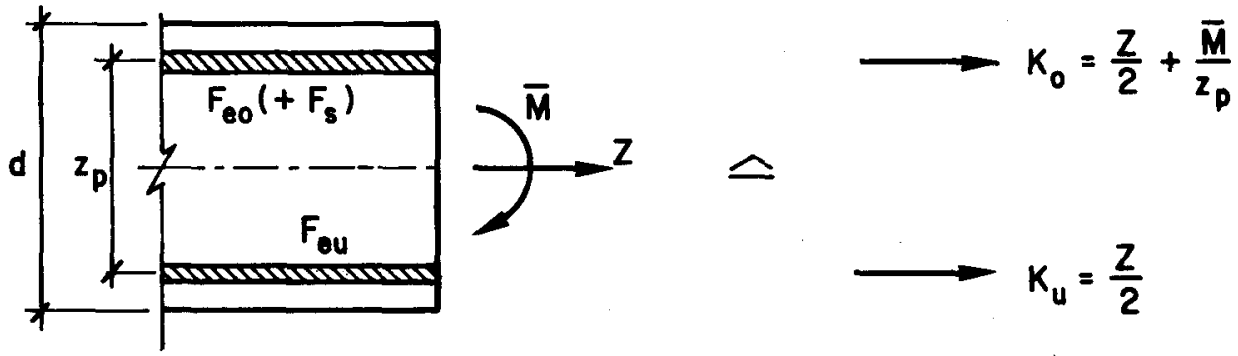

Überlagerung der Kräfte aus exzentrisch mit $e=z_{p} / 2$ ongreifender Querzugkraft und aus Querbiegung

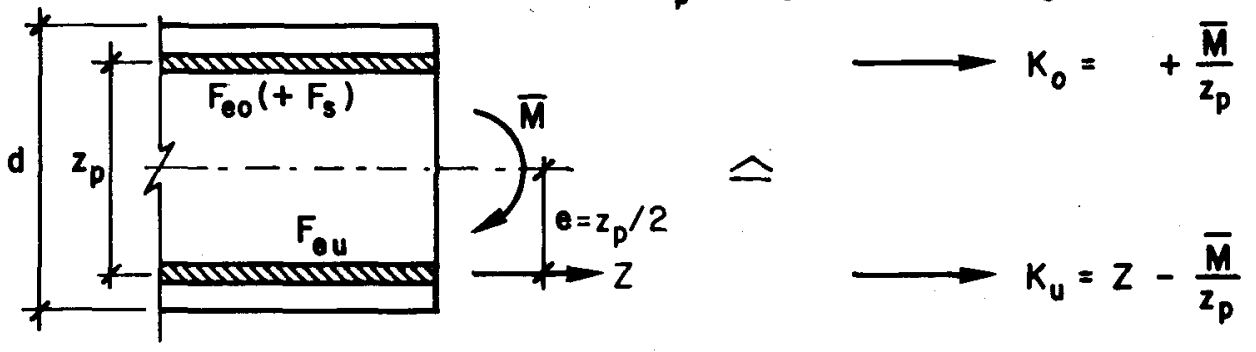

Überlagerung der Kräfte ous zentrisch angreifender Querzugkraft und aus Querbiegung
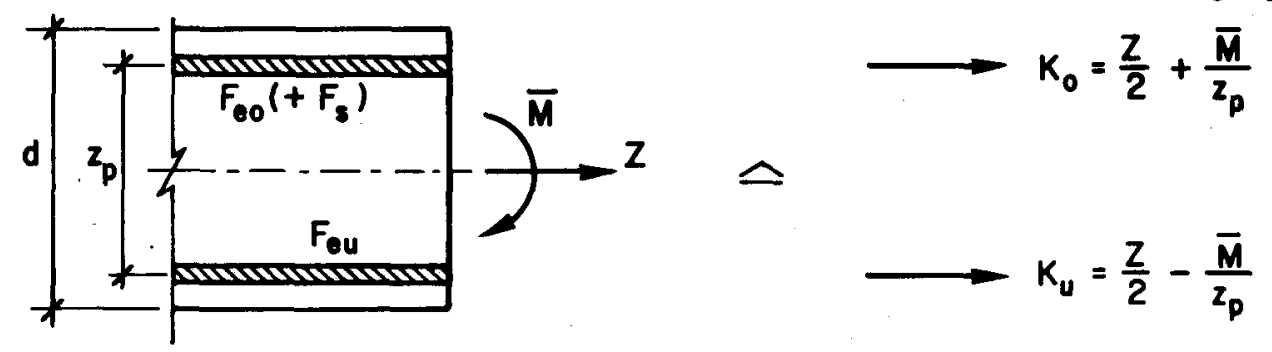

Überlogerung der Kröfte aus exzentrisch mit $e=z_{p} / 4$ angreifender Querzugkraft und aus Querbiegung
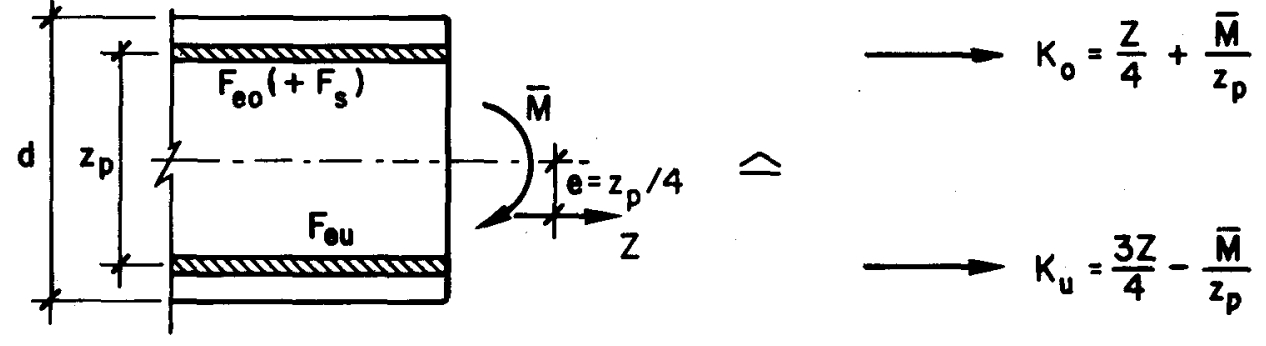


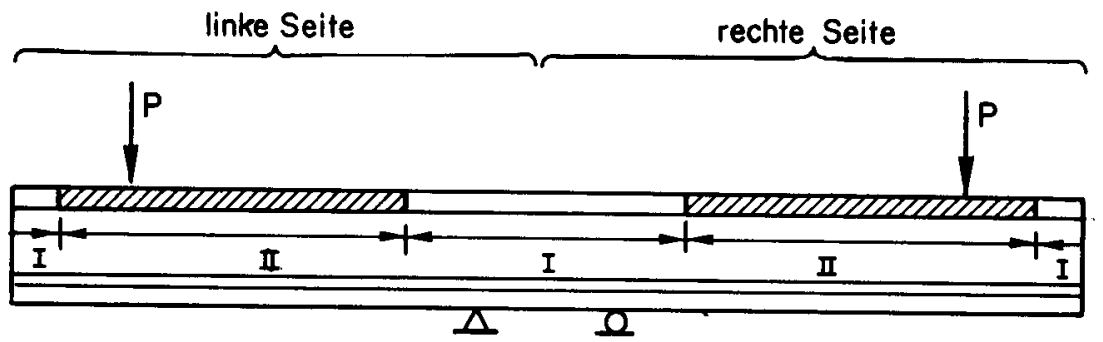

Bereich I Keine Querbeanspruchung

Bereich II Längsschub allein

theoretisch erforderliche Fliesskraft

$\square$ konstruktive Mindestarmierung
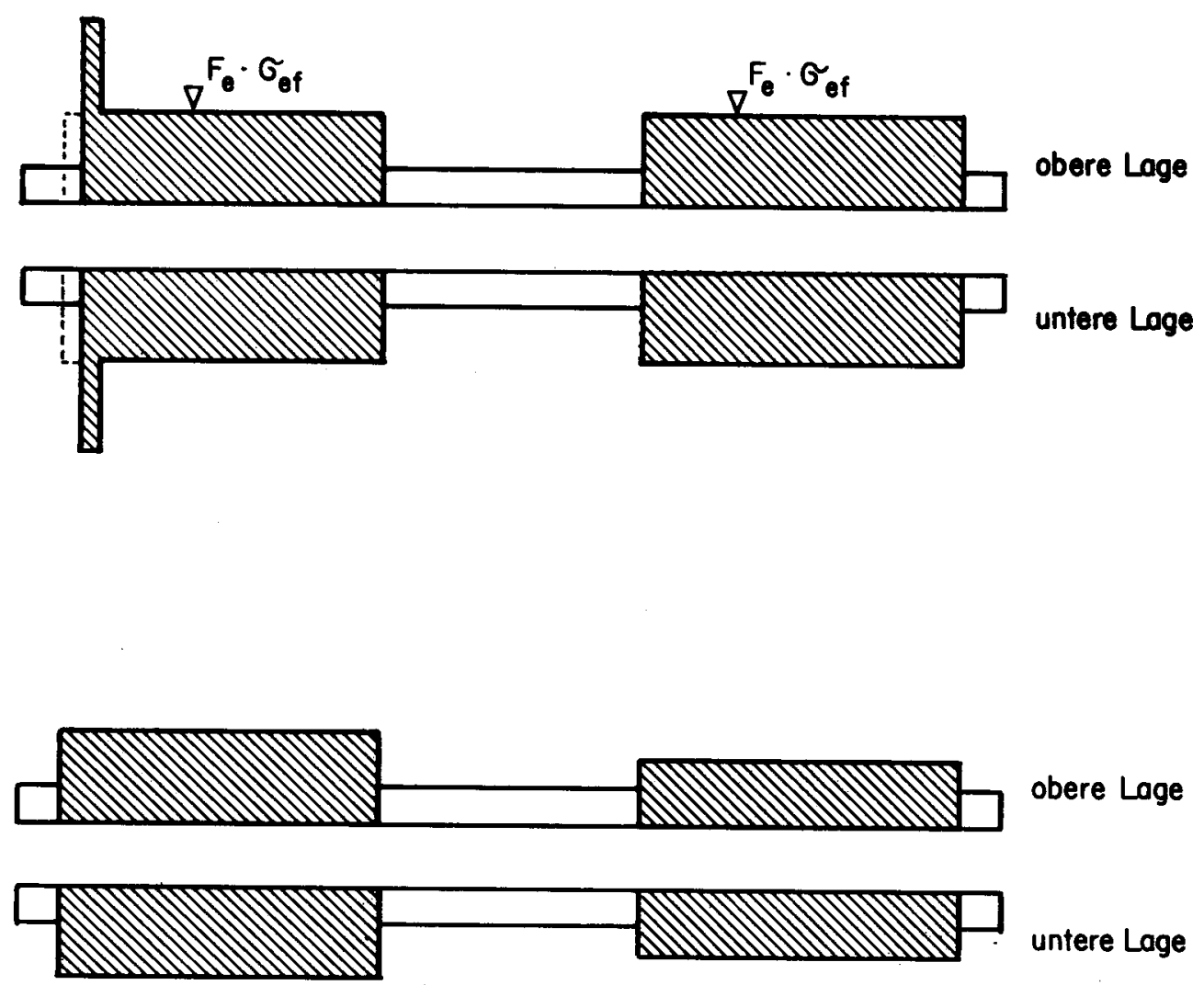

Um $30 \%$ vergrösserte

Armierung gegenüber der

rechten Seite 


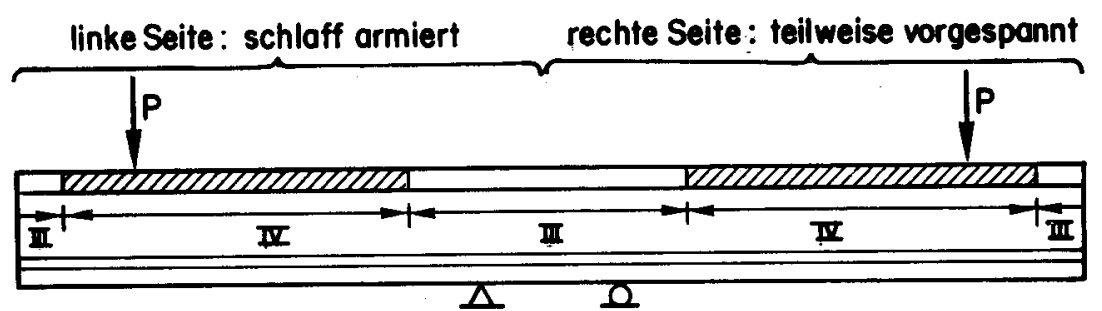

Bereich III Reine Querbiegung

Bereich II Löngsschub + Querbiegung

theoretisch erforderliche Fliesskraf $t$

$\square$ konstruktive Mindestarmierung

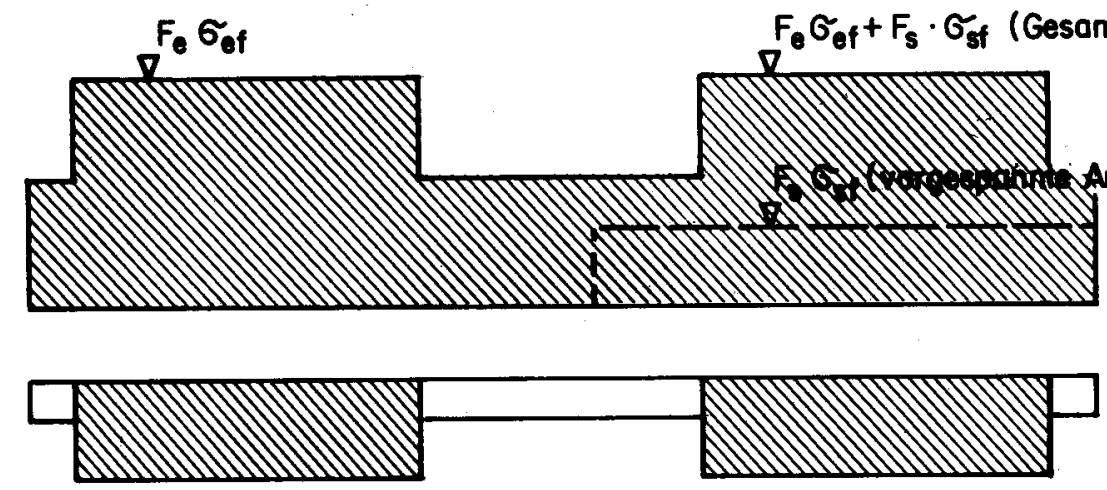

\section{obere Lage (Querbiegezugseite)}

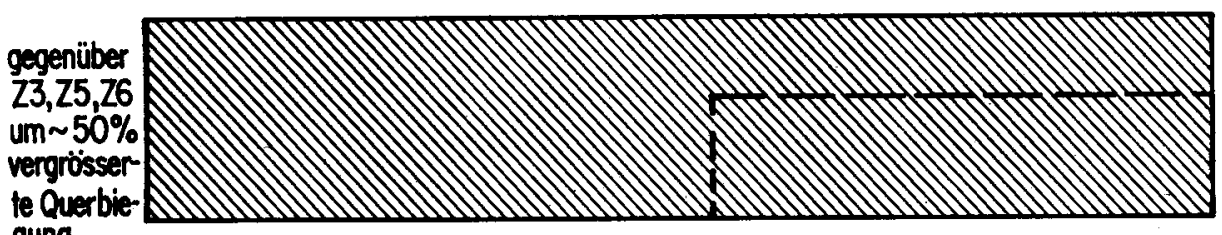

obere Loge gung

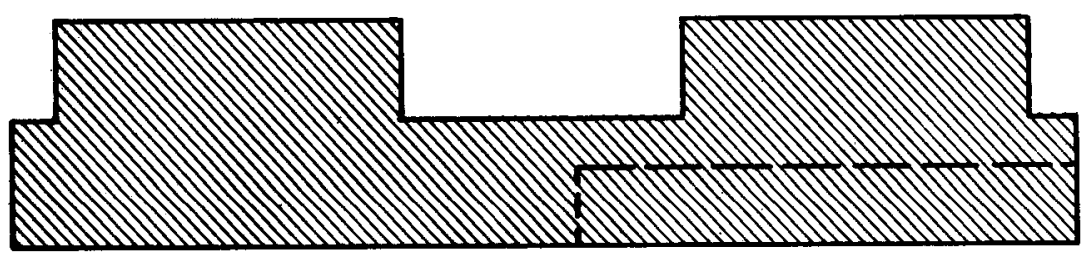

obere Loge

untere Lage

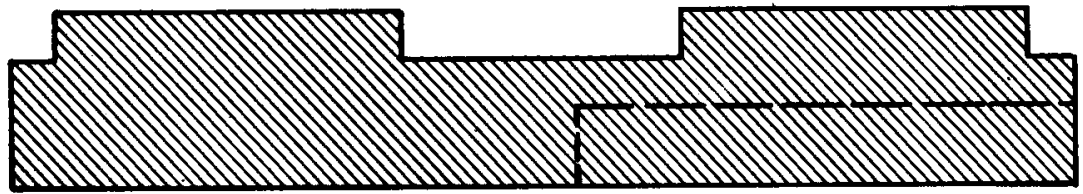

obere Loge

Bild 4-b: Fliesskräfte der Plattenquerarmierung

untere Loge 


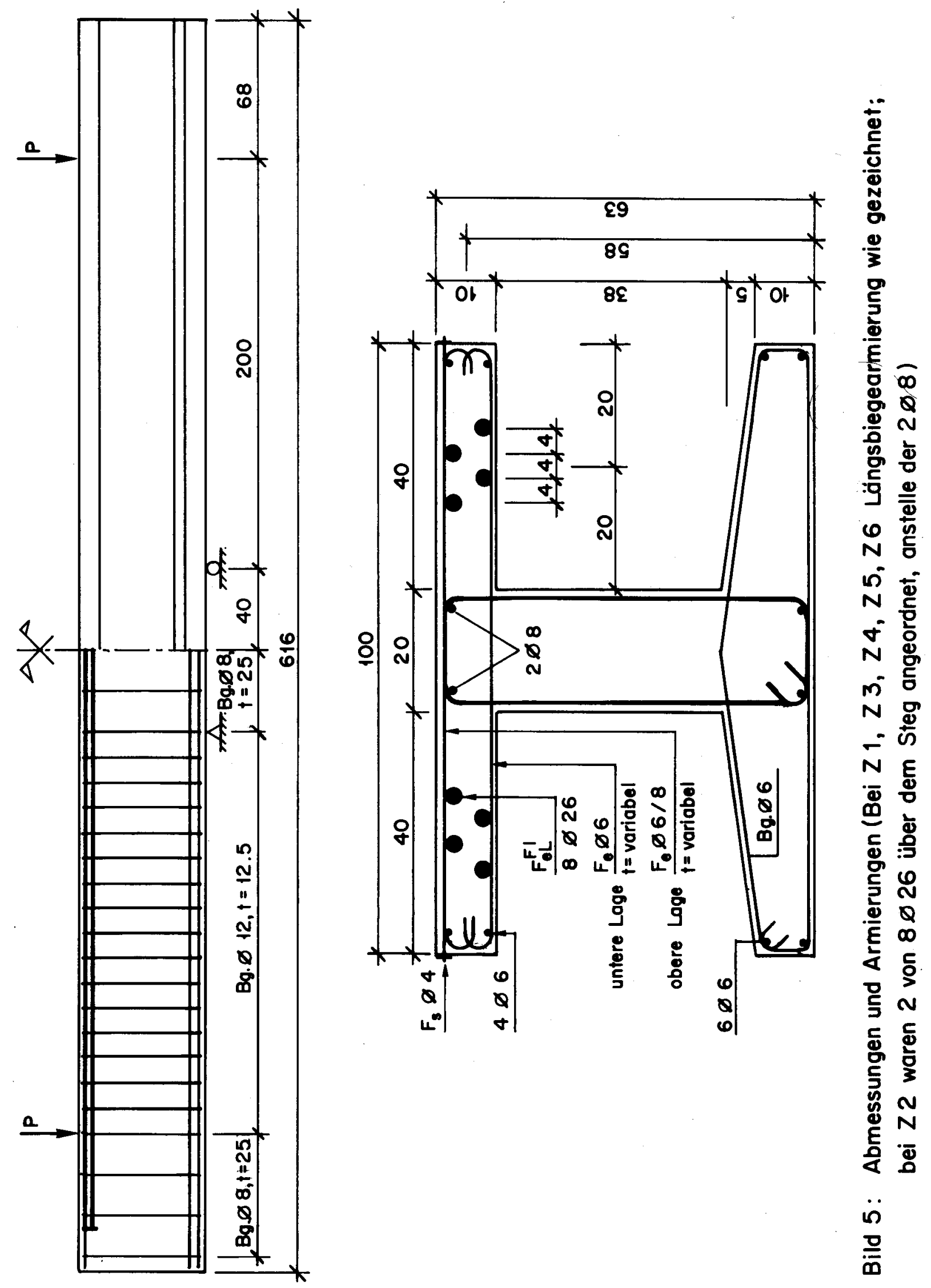


N

$N$
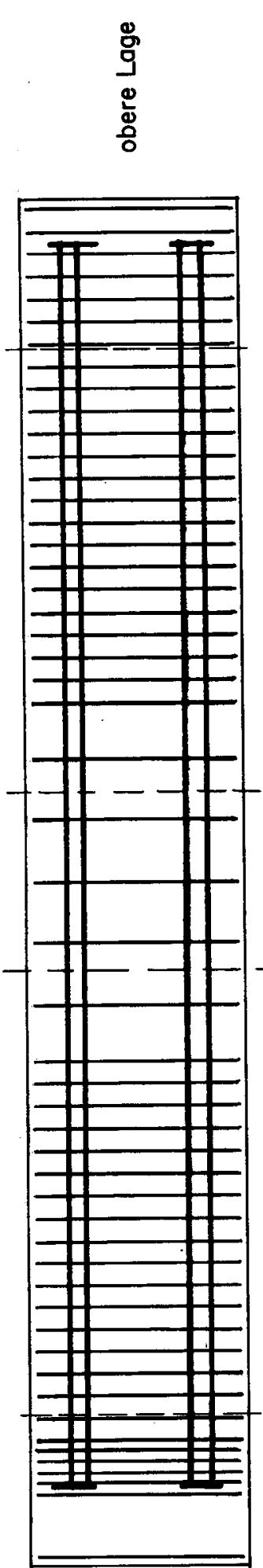

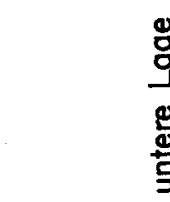

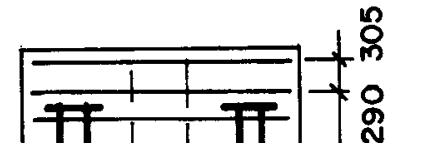
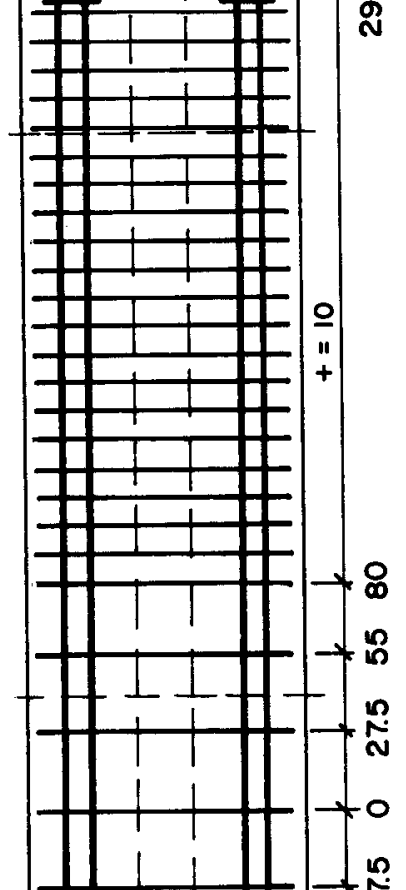

丹1 $1+1-{ }_{-\infty}^{n}$
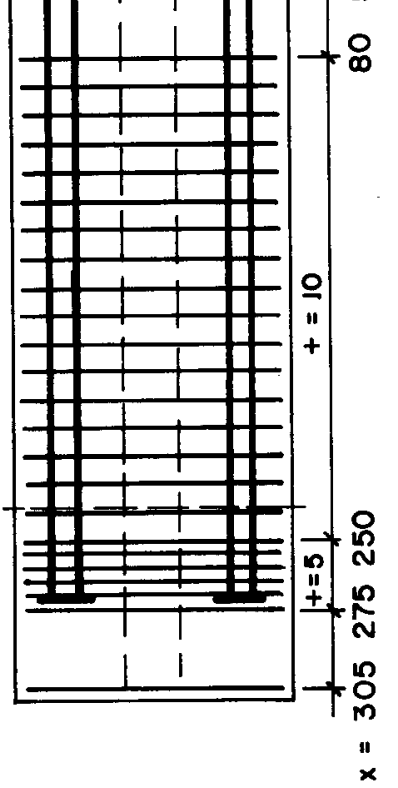

$\stackrel{9}{9}$
9
$\stackrel{0}{0}$
$\stackrel{0}{0}$

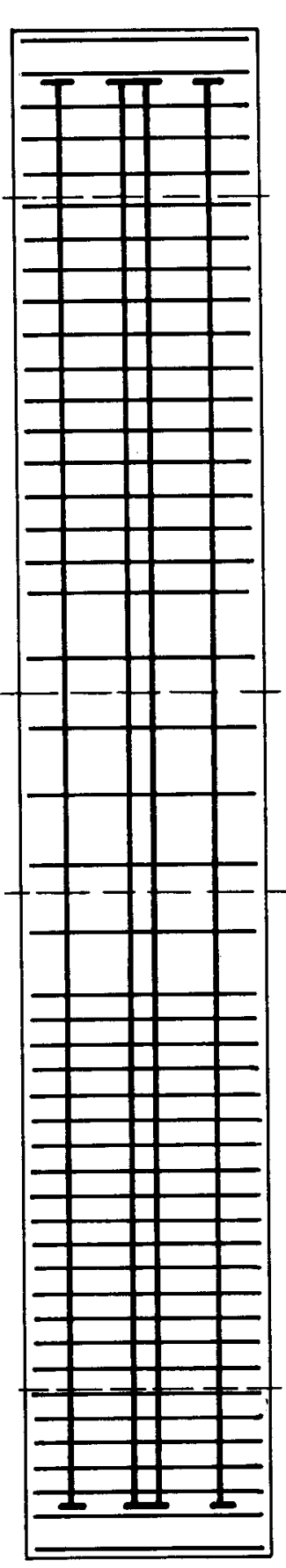

$\frac{8}{\stackrel{g}{g}}$

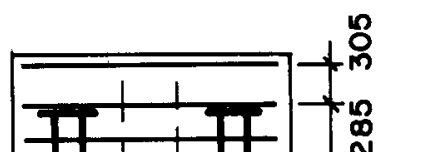

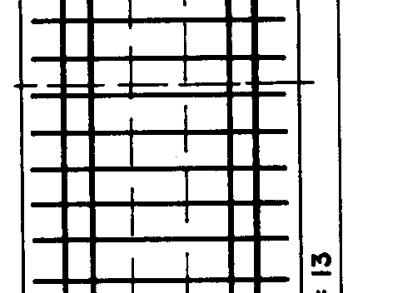
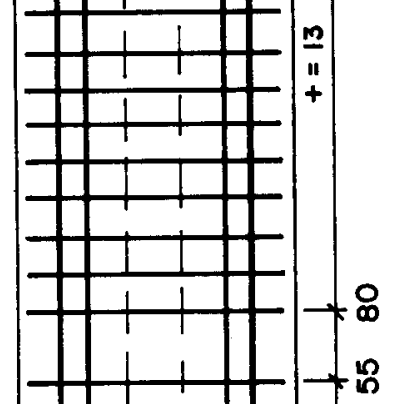

†正H十

$\grave{Q}$

Q

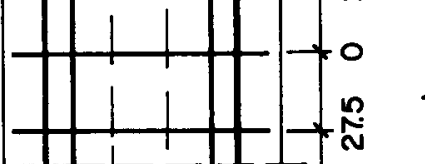

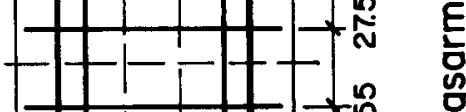
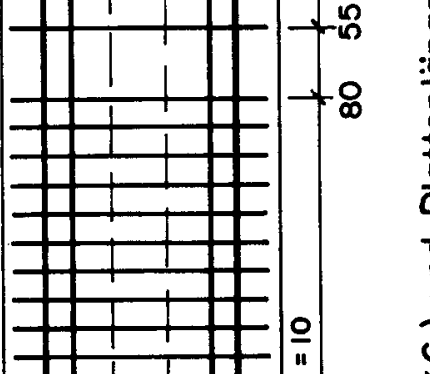

$7^{\circ} \div$

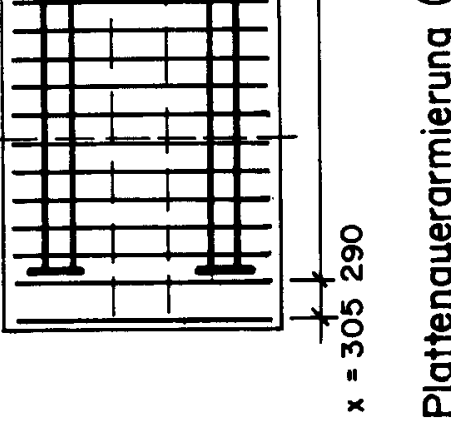

í

음 


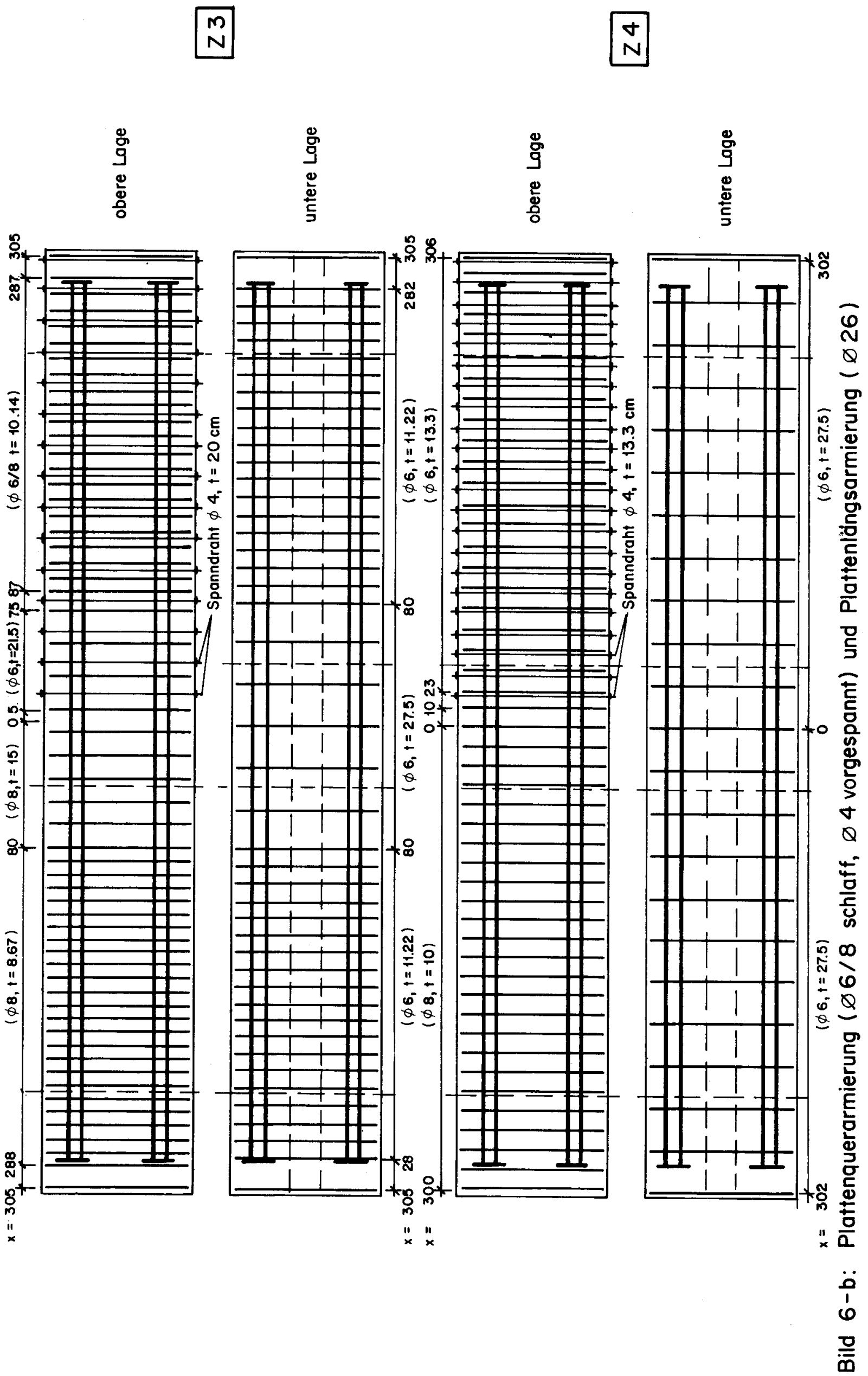


$n$
$N$

0
$N$

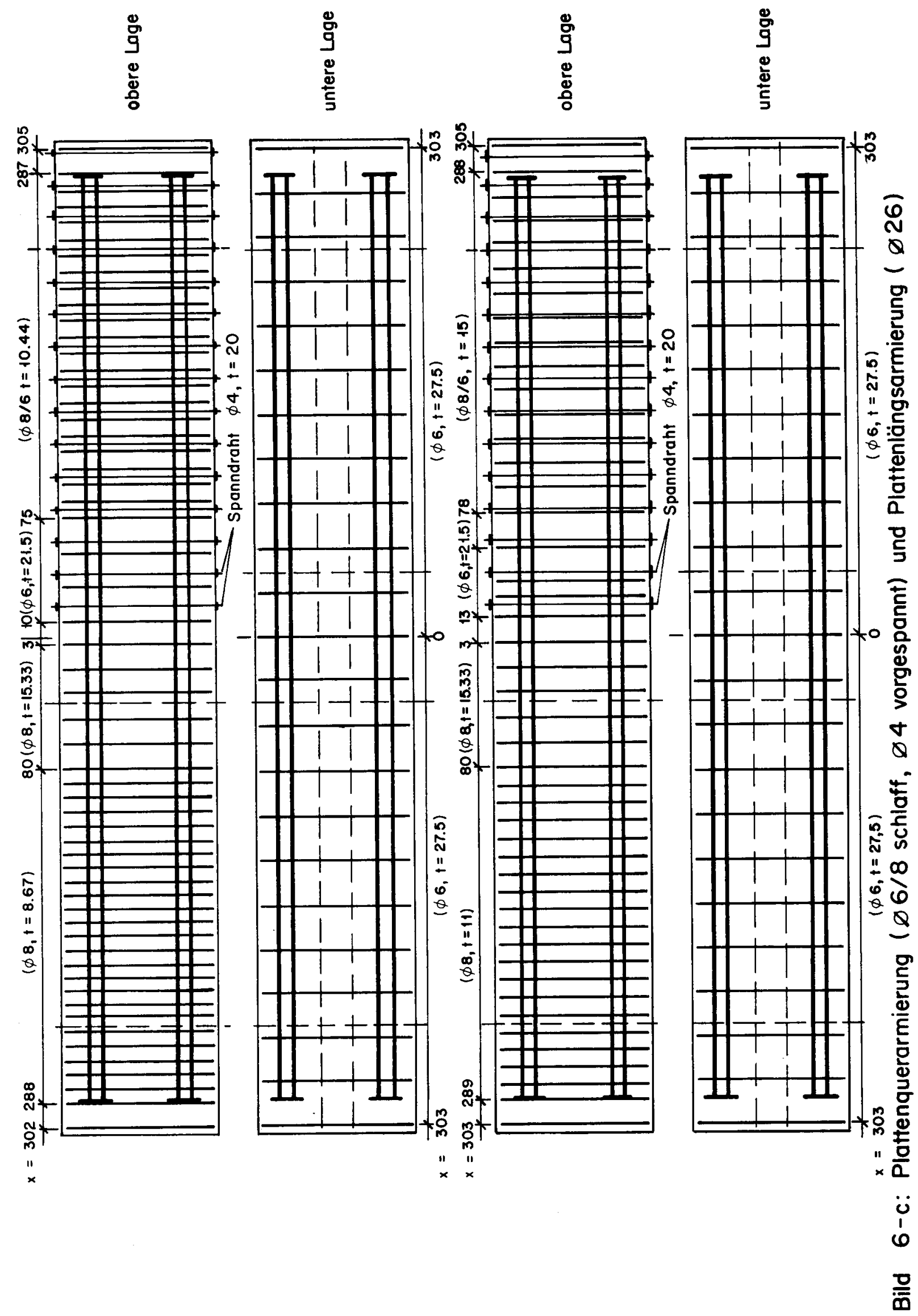



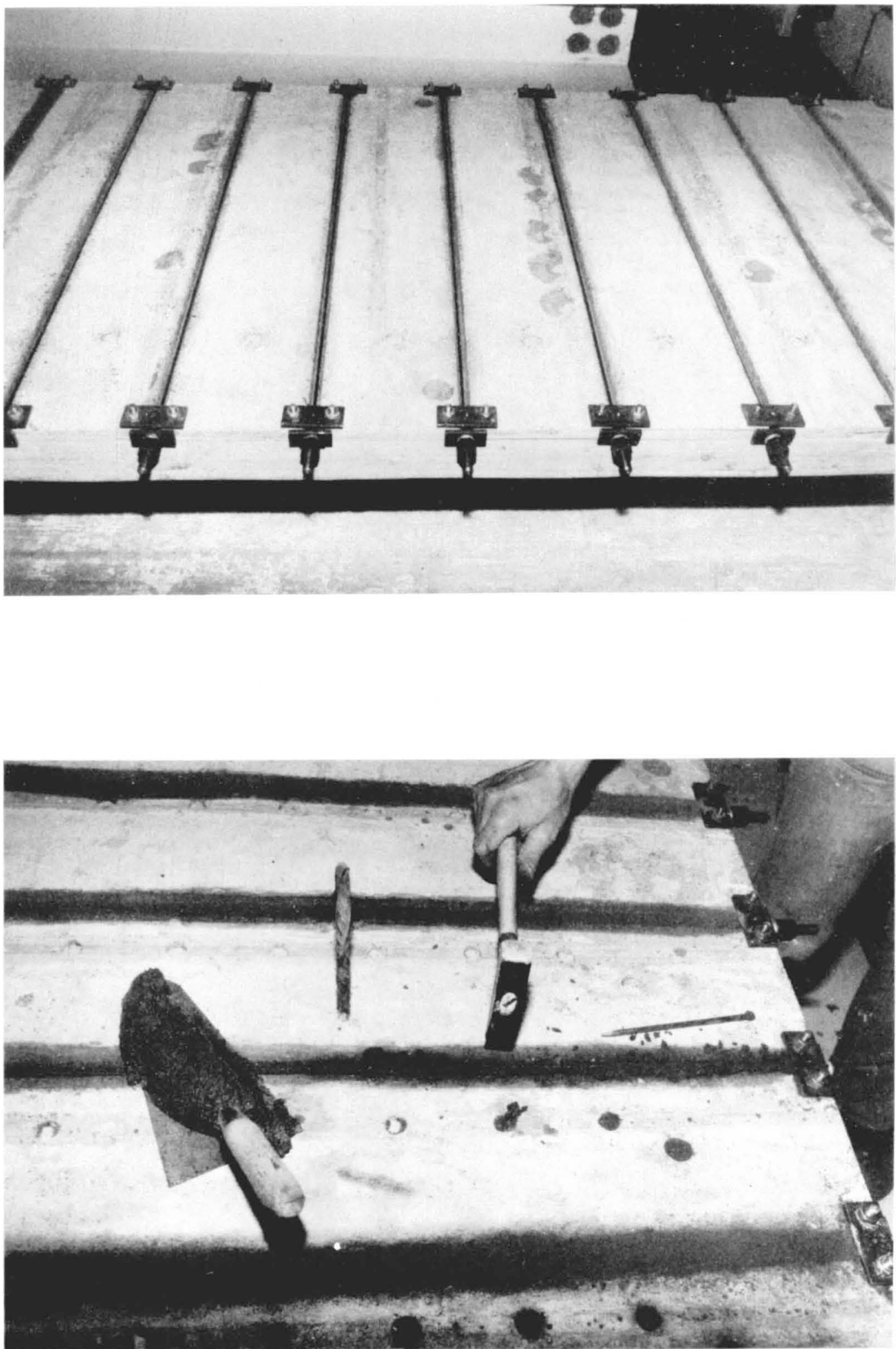

Bild 7: Quervorspannung der Zugplatte, vor und beim „Injizieren" 


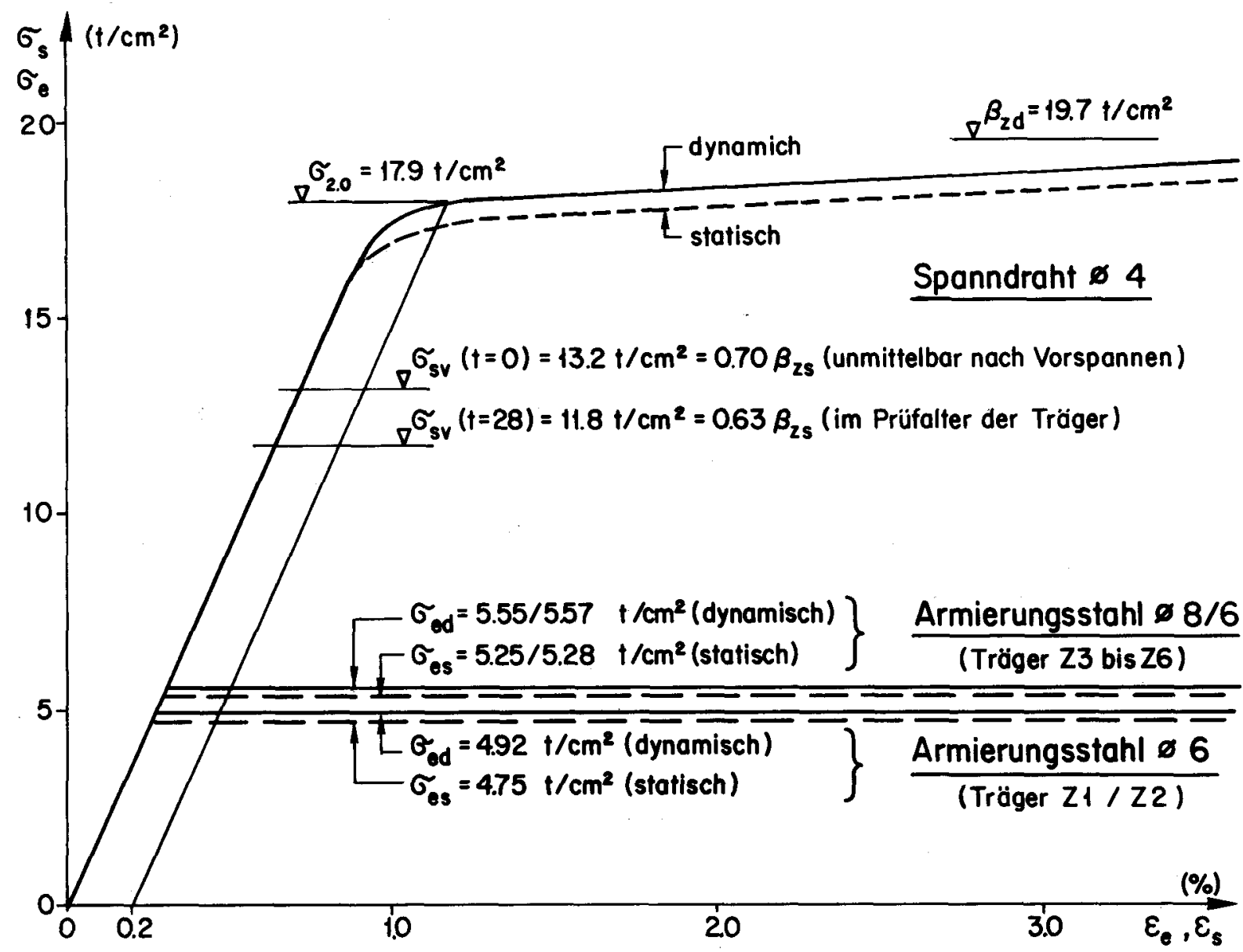

Bild 8: $\sigma-\varepsilon-$ Diagramme der Plattenquerarmierung

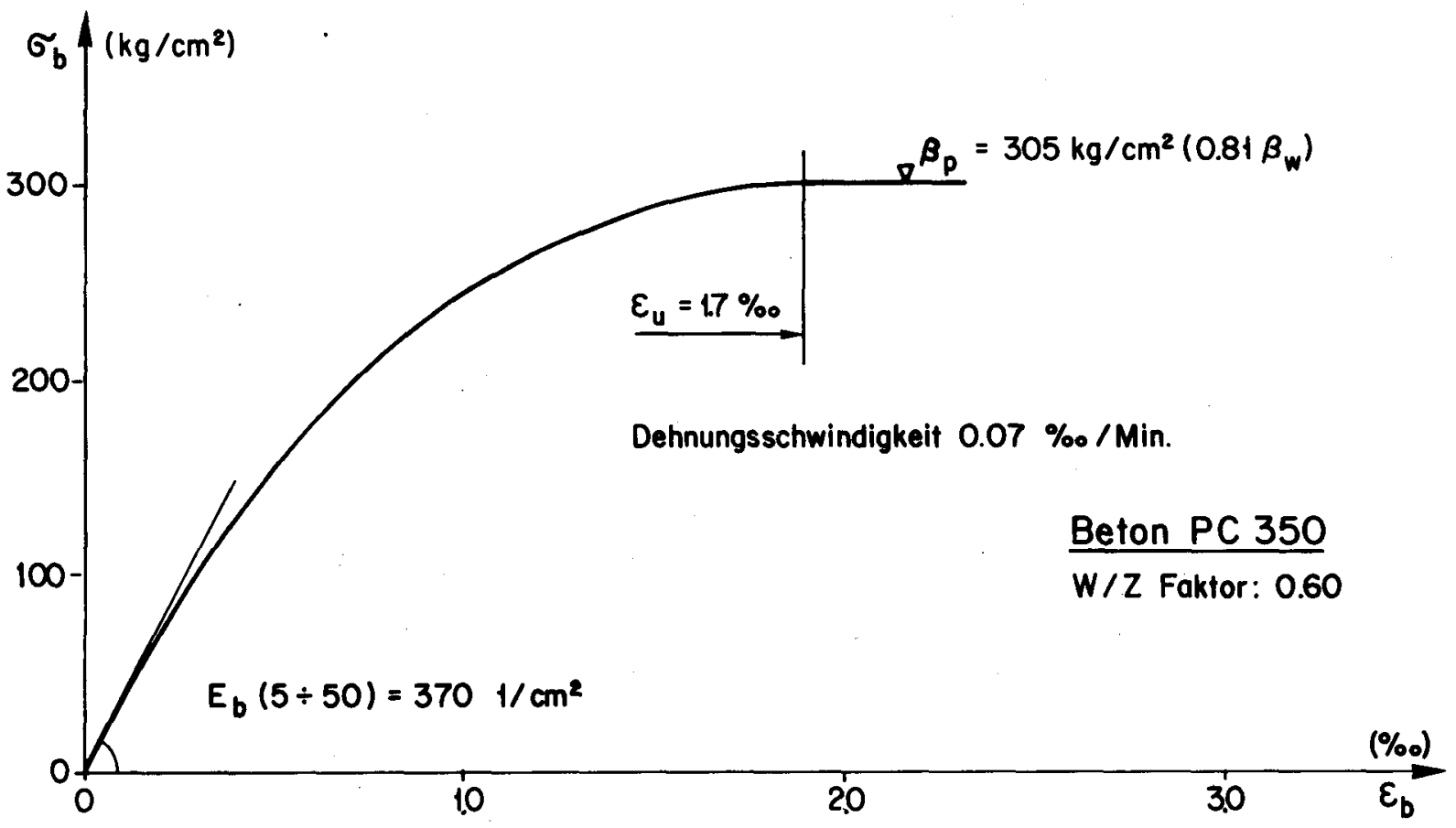

Bild 9: $\sigma-\varepsilon-$ Diagramm des Betons (nach 28 Tagen) 
Laststellung P für Löngsbeanspruchung :

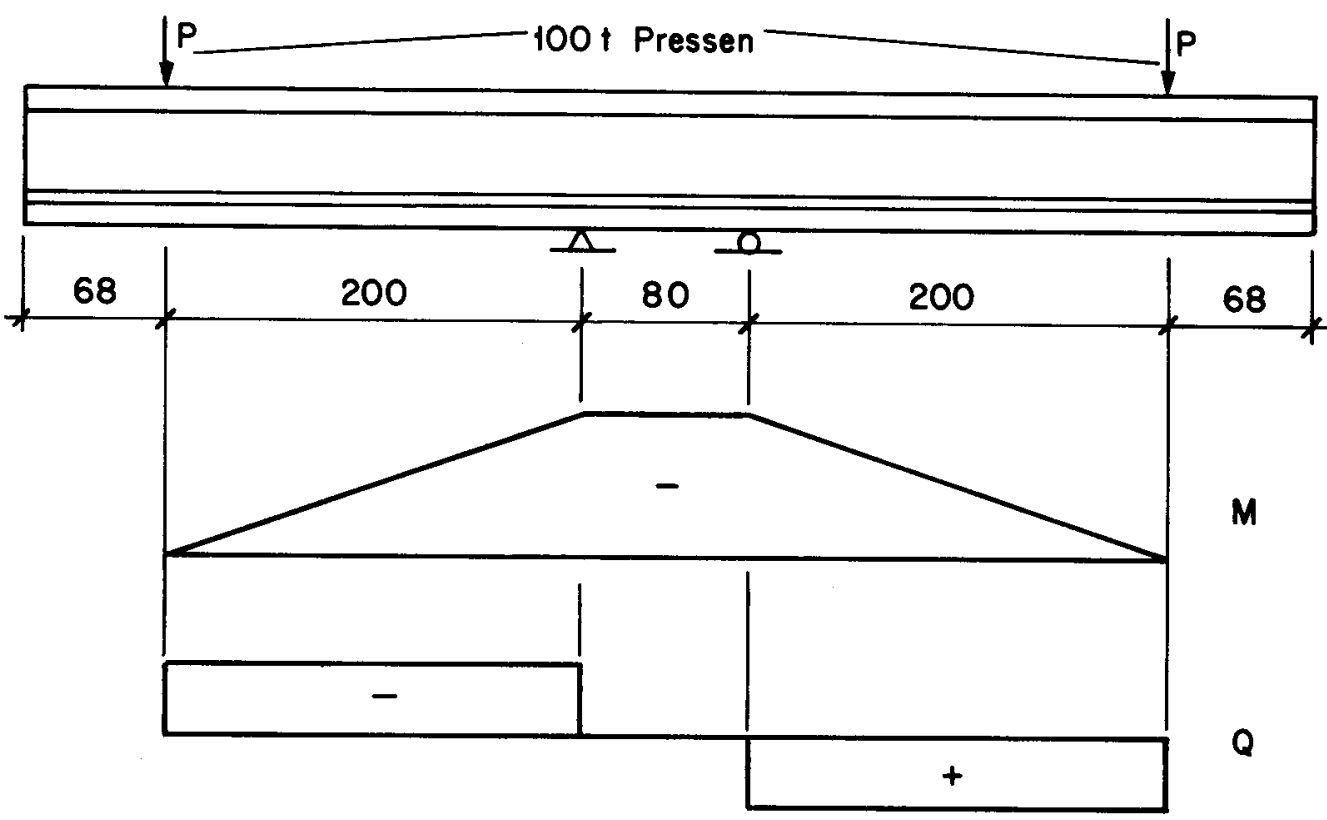

Laststellung $\overline{\mathrm{P}}$ für Querbeanspruchung:
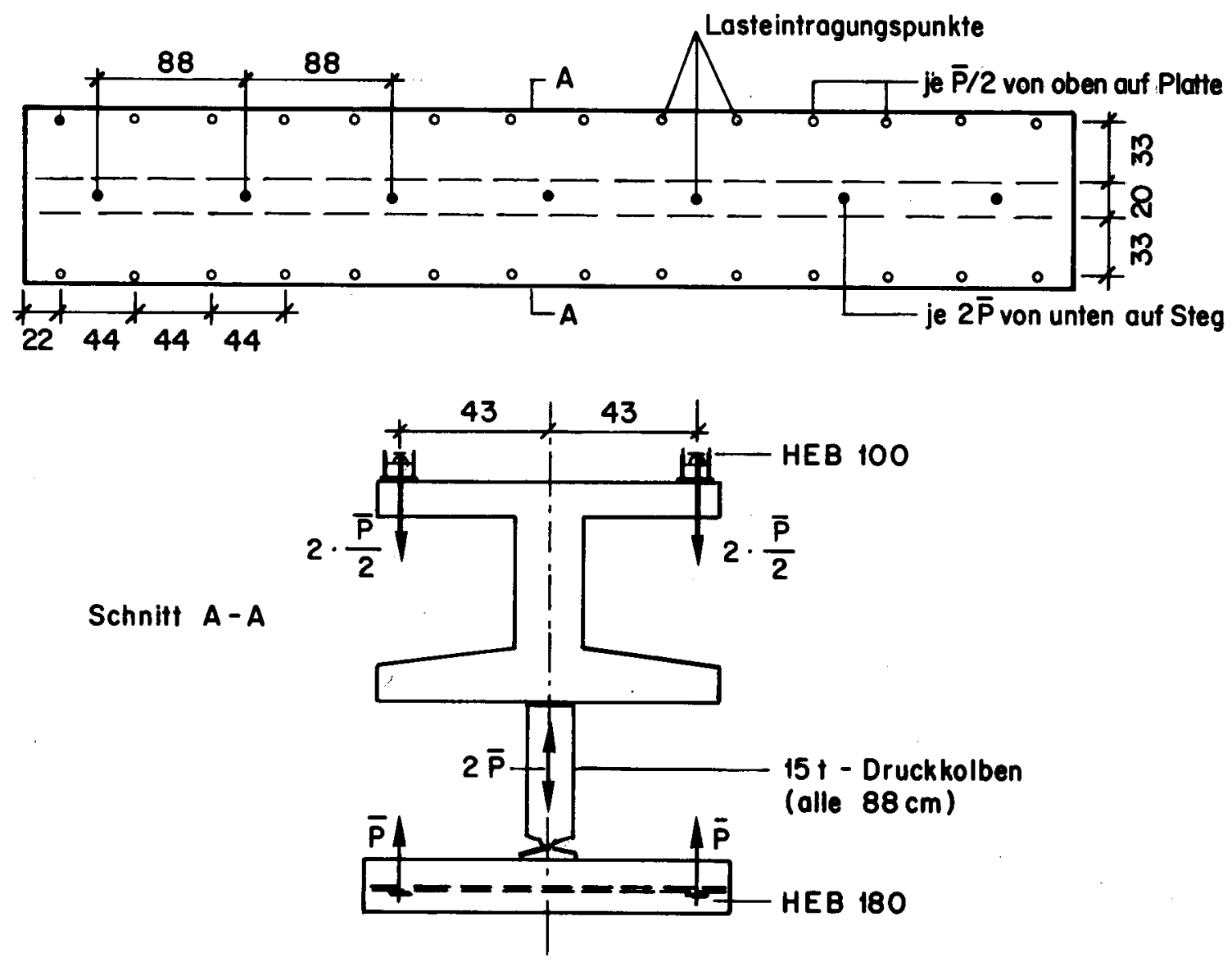

Bild 10: Belastungsanordnung für Längs-und Querbeanspruchung 


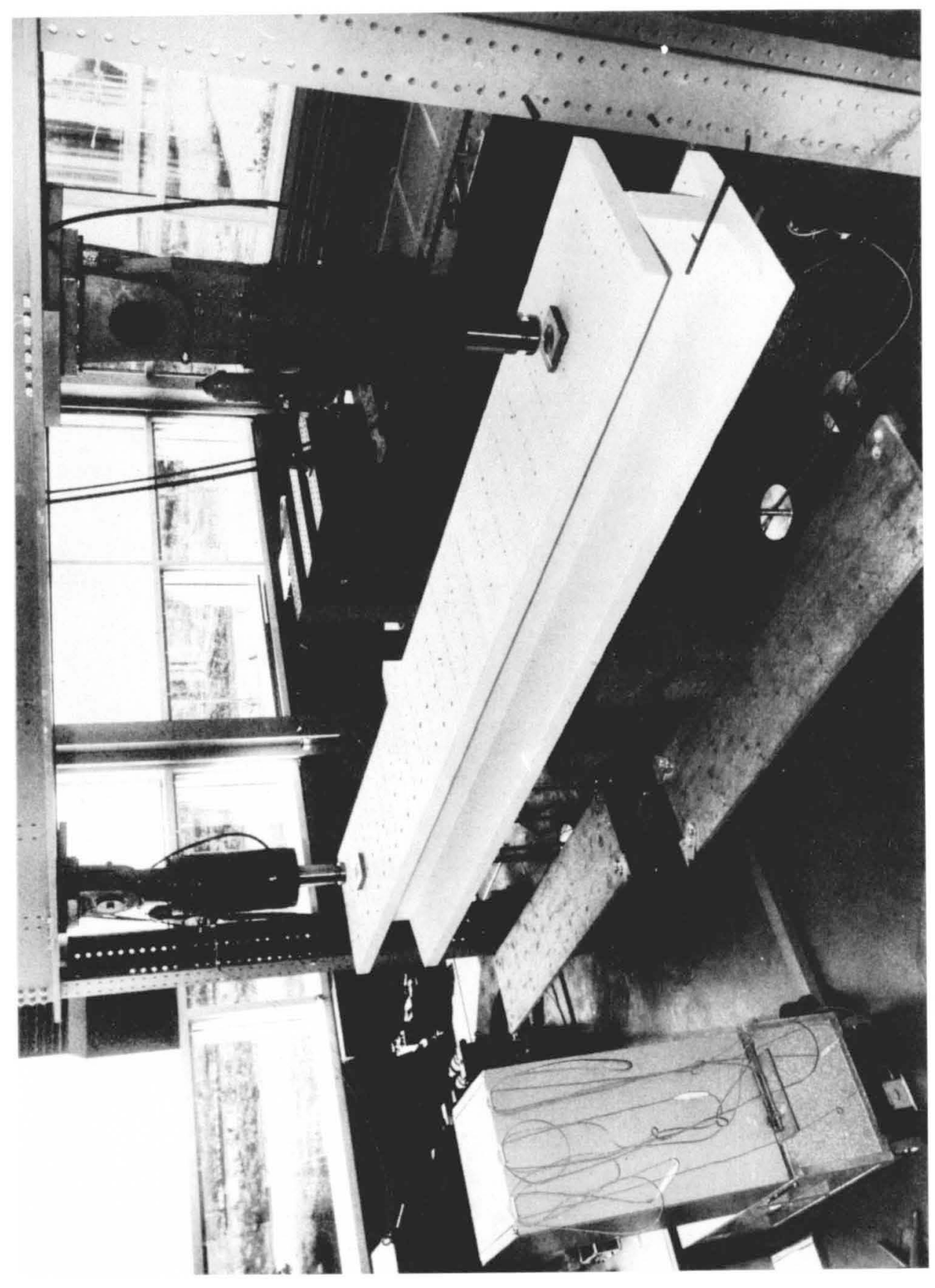

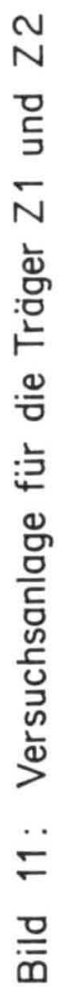




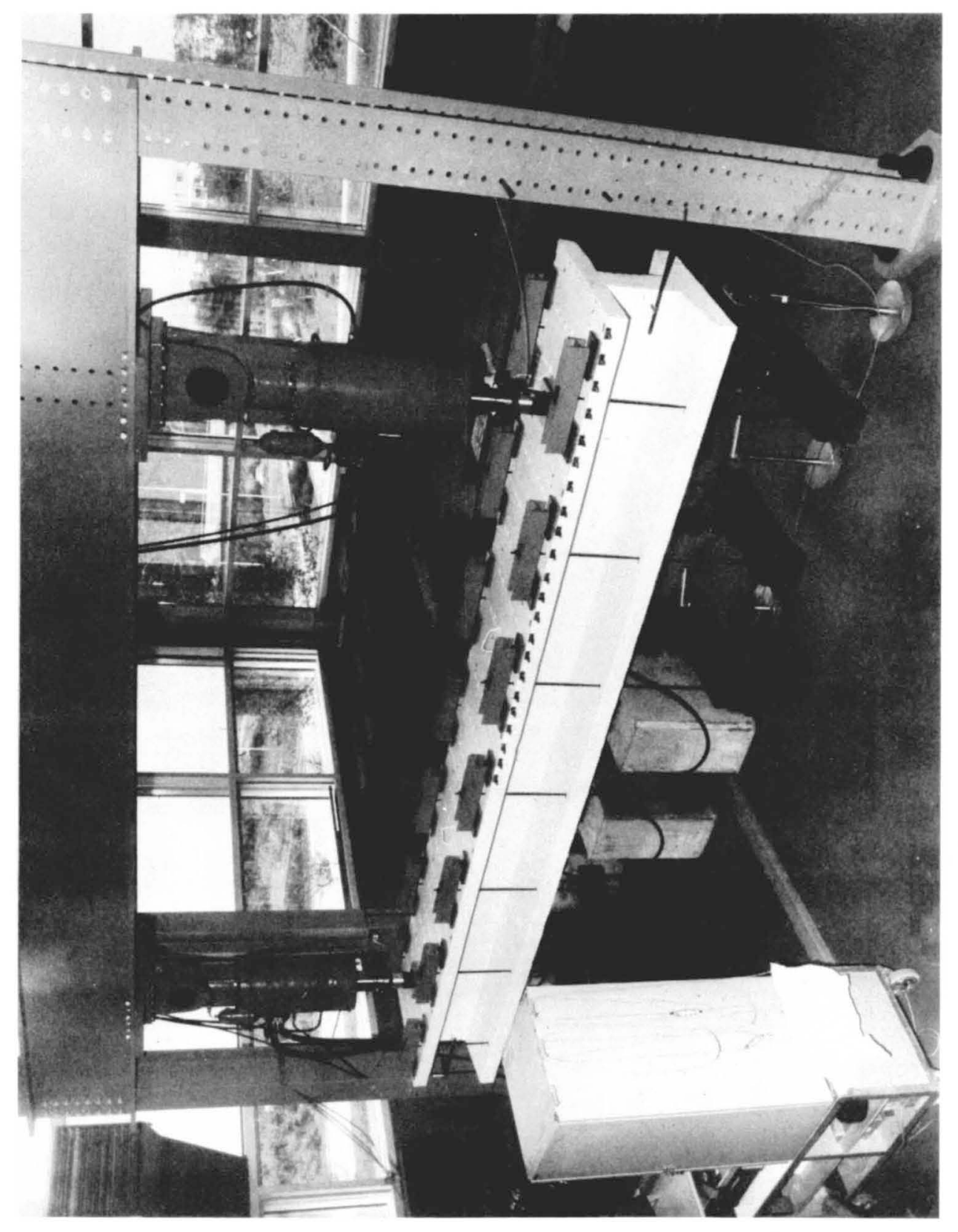

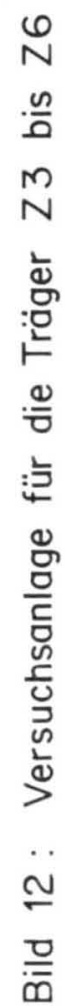



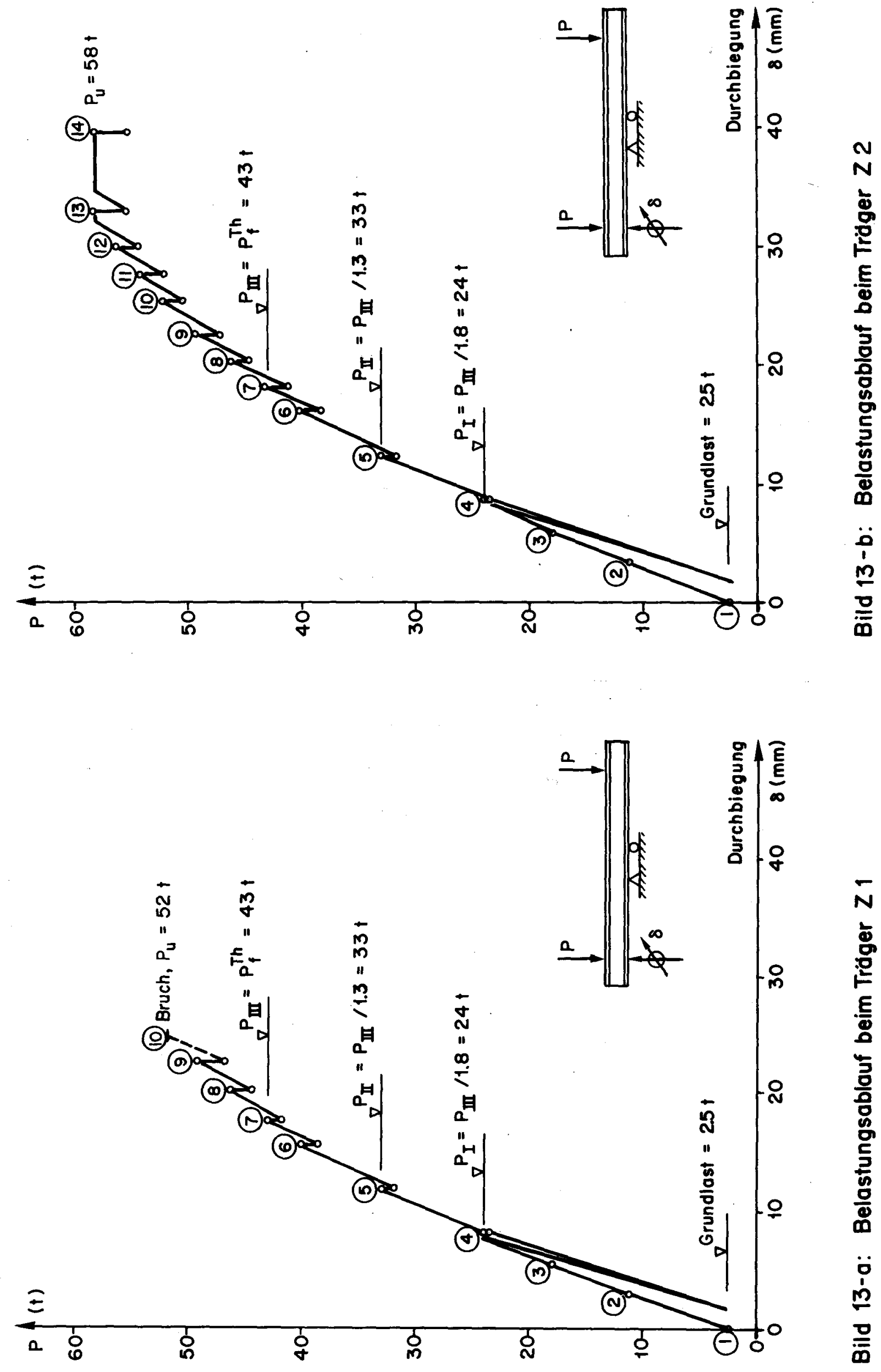

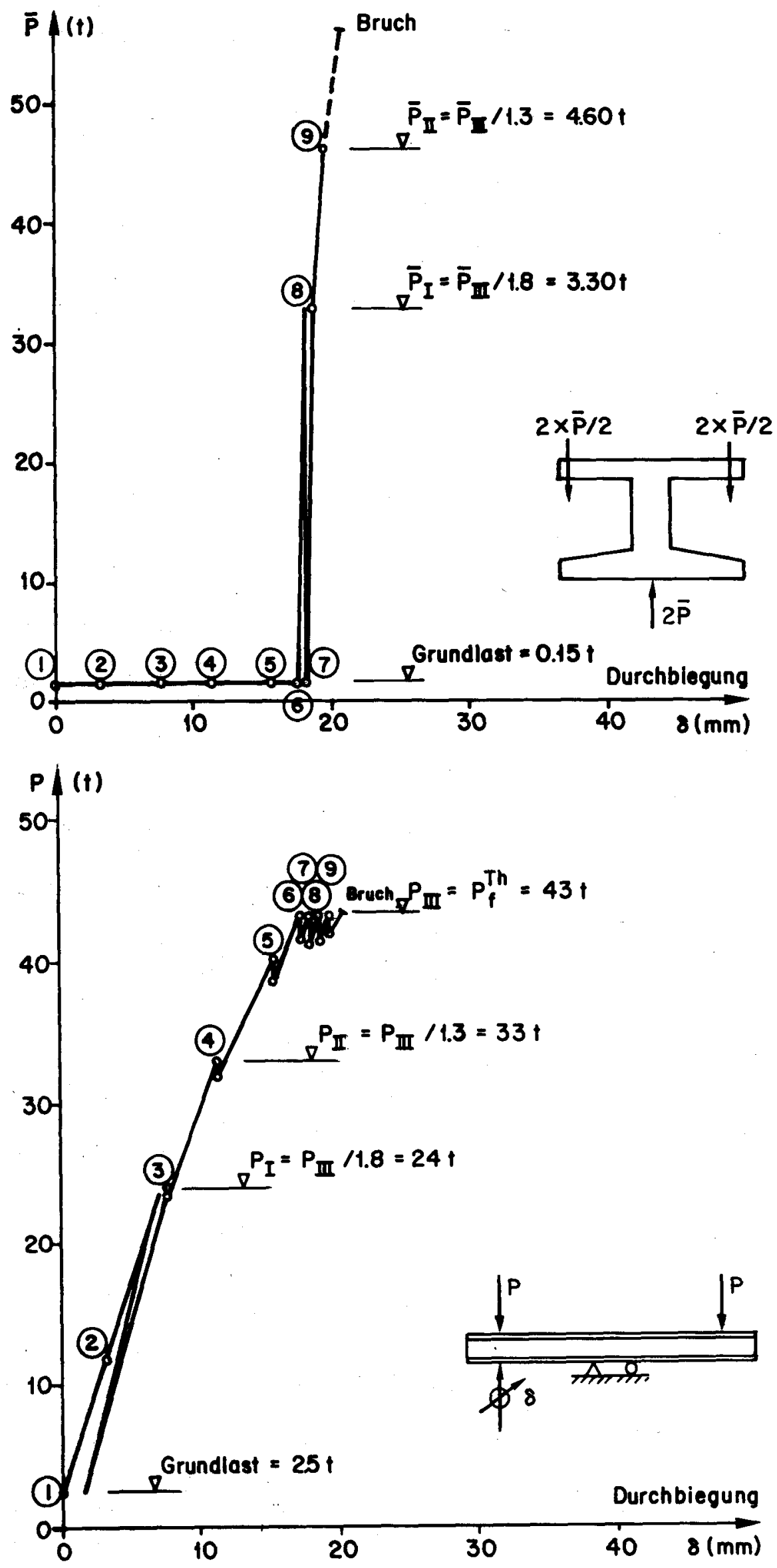

Bild 14: Belastungsablauf beim Trüger Z4 

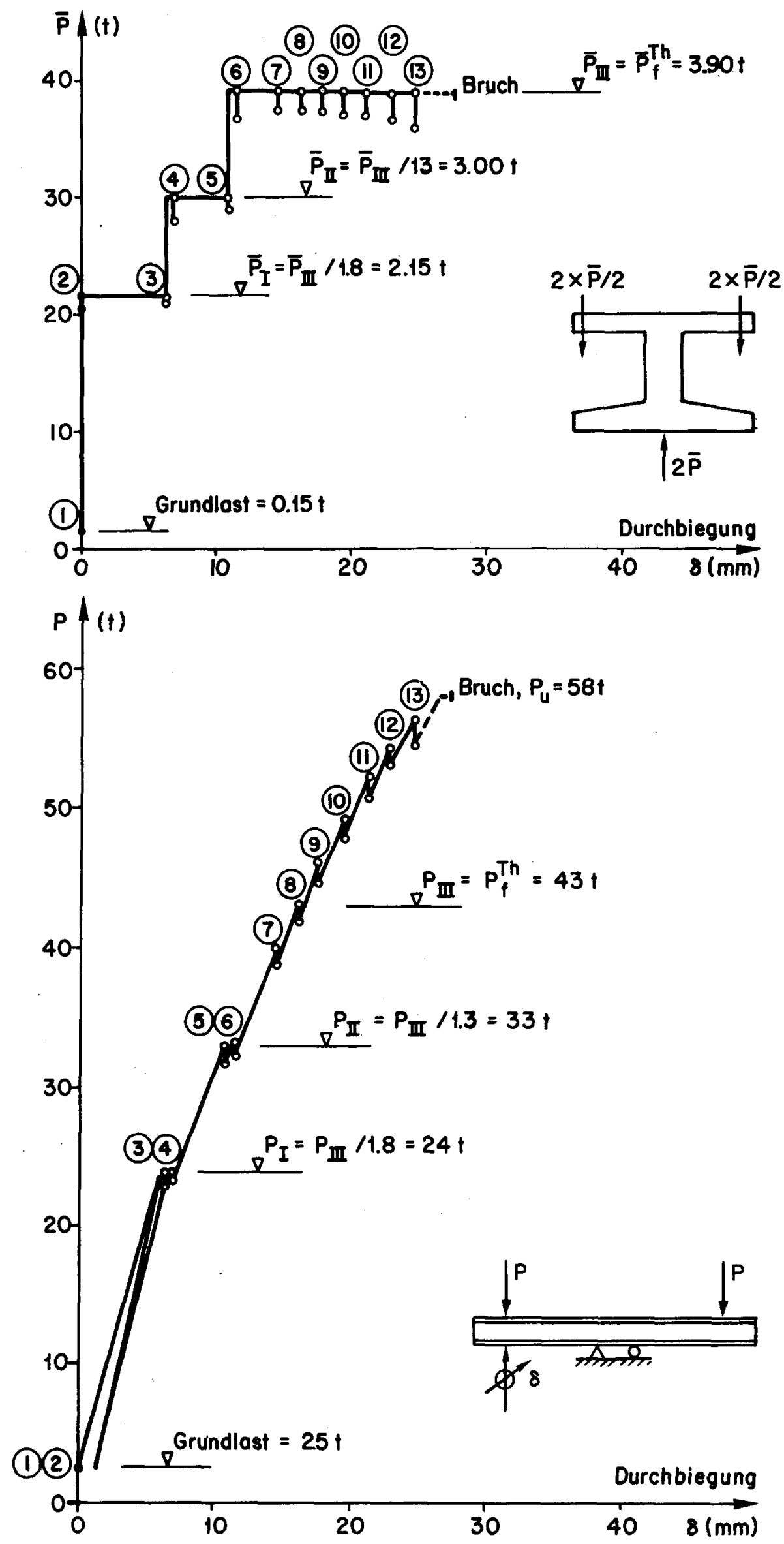

Bild 15: Belastungsablauf beim Trdger $Z 5$ ( $Z 3$ und $Z 6$ analog) 


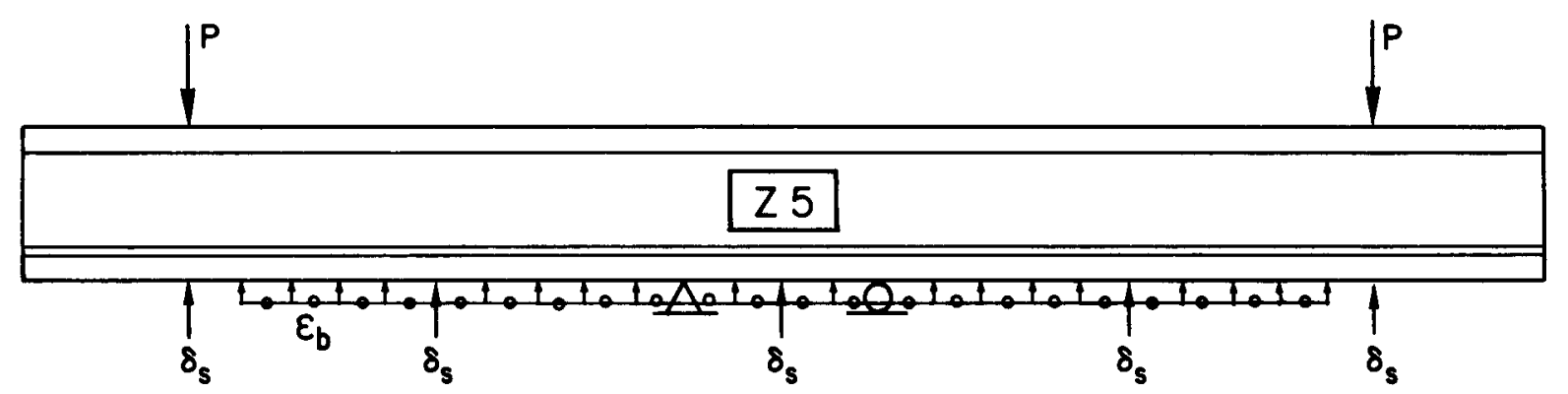

Durchbiegungen $\delta_{s}$

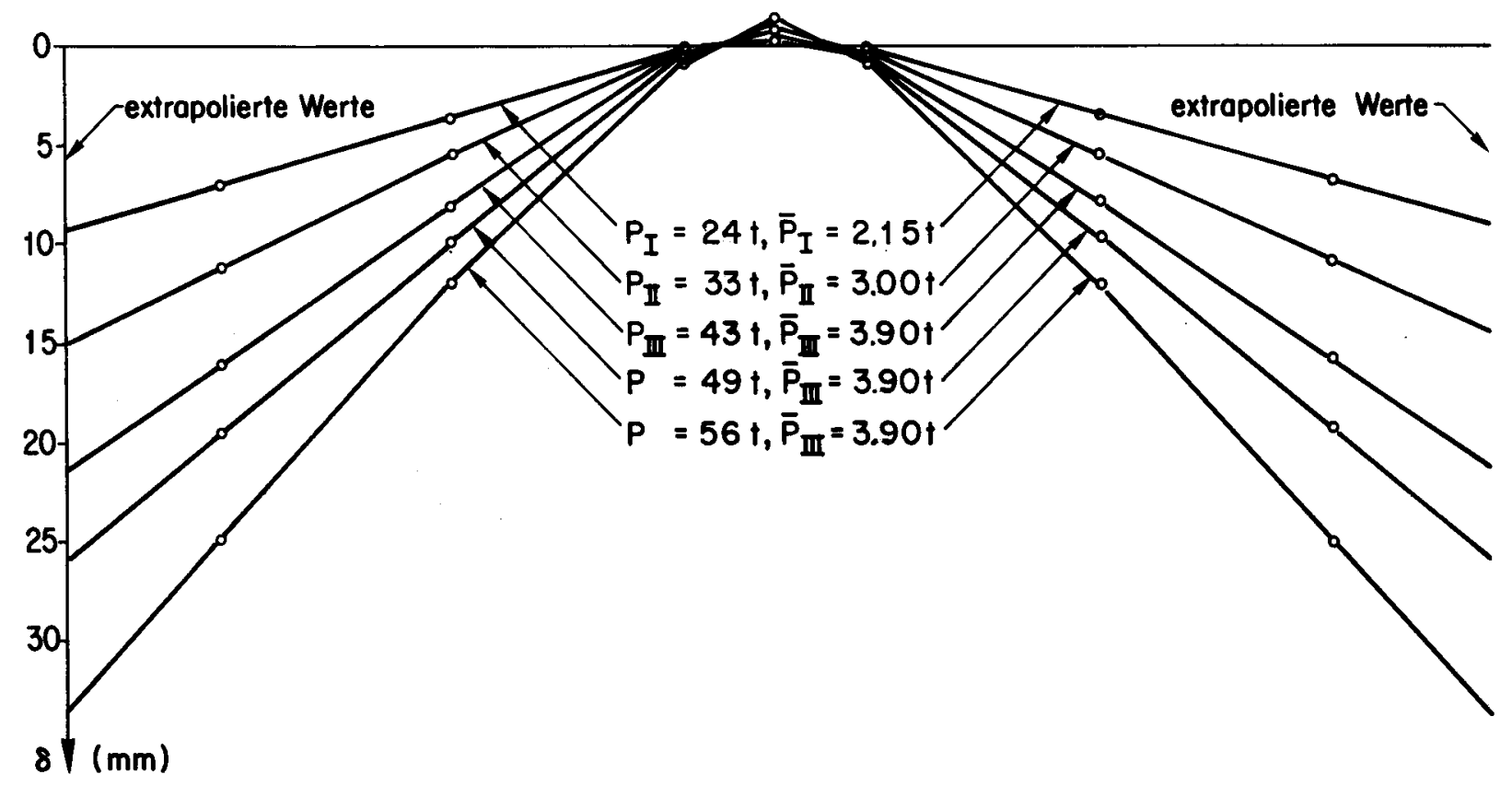

Betonstauchungen $\varepsilon_{b}$ in der Druckplatte (Unterseite)

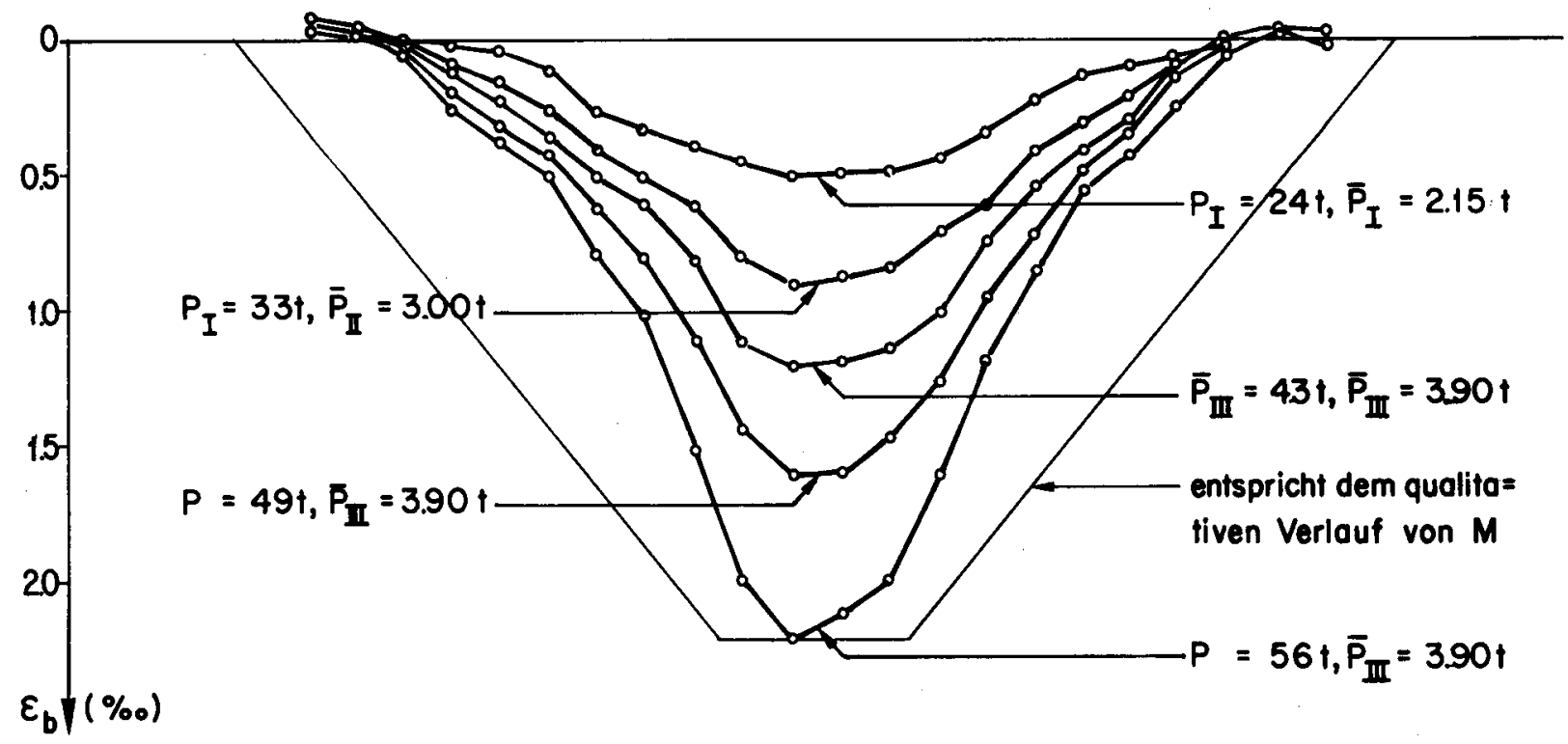

Bild 16: Beispiel für die Durchbiegungen und die Stauchungen der Druckplatte 

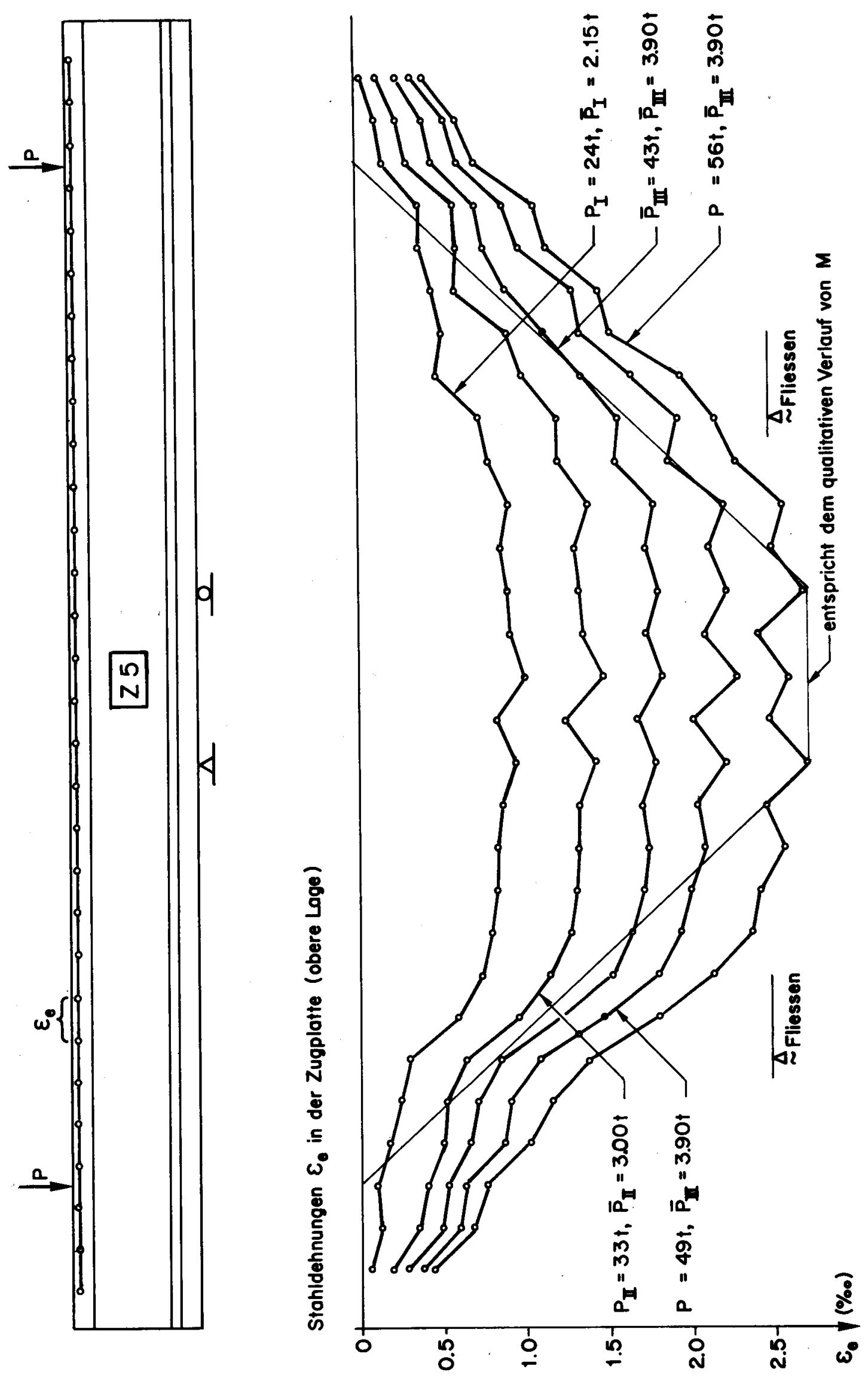

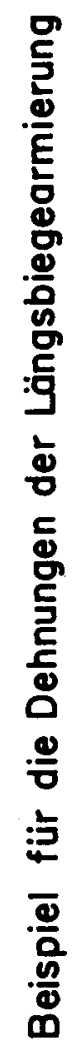

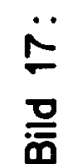




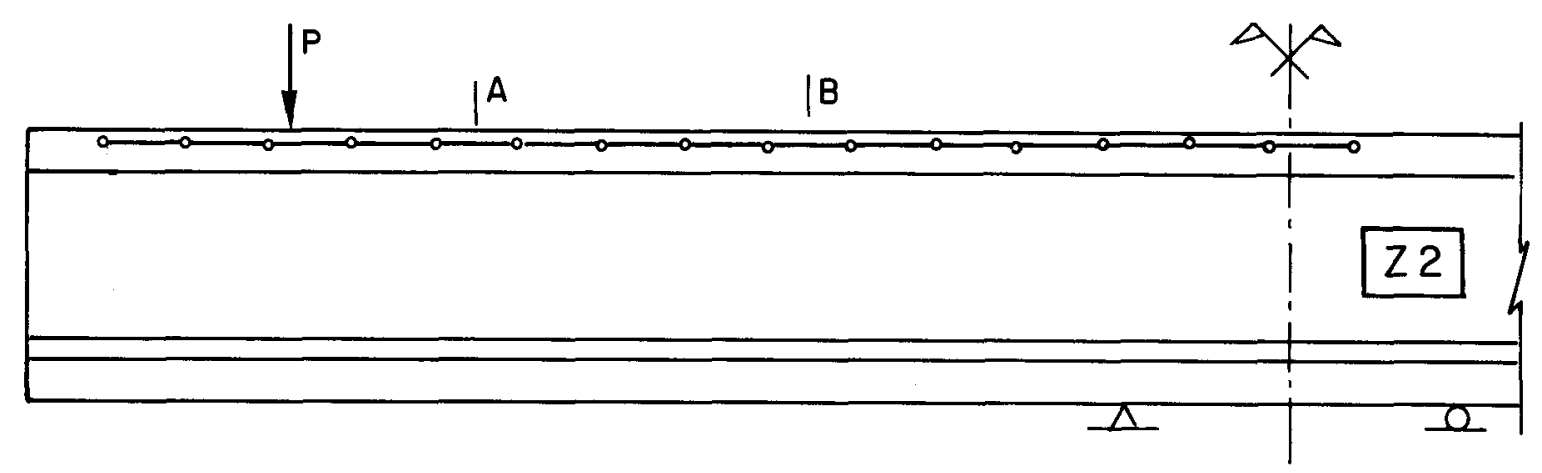

Stahldehnungen $\varepsilon_{\mathrm{e}}$ in der Zugplatte (obere Loge)
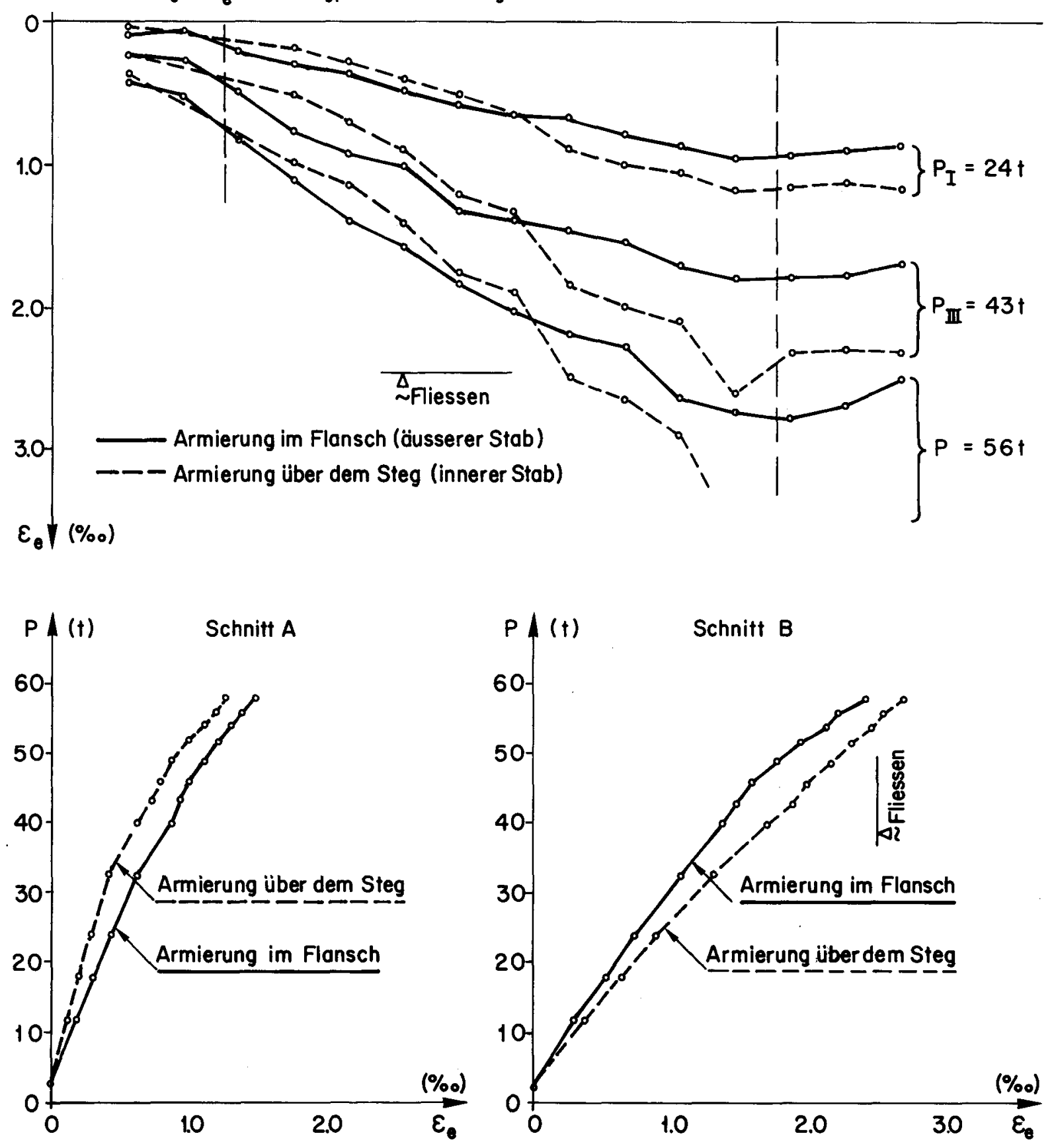

Vergleich der Stahldehnungen in den Schnitten A und B

Bild 18: Dehnungen der oberen Längsbiegearmierung beim Träger $Z 2$ 


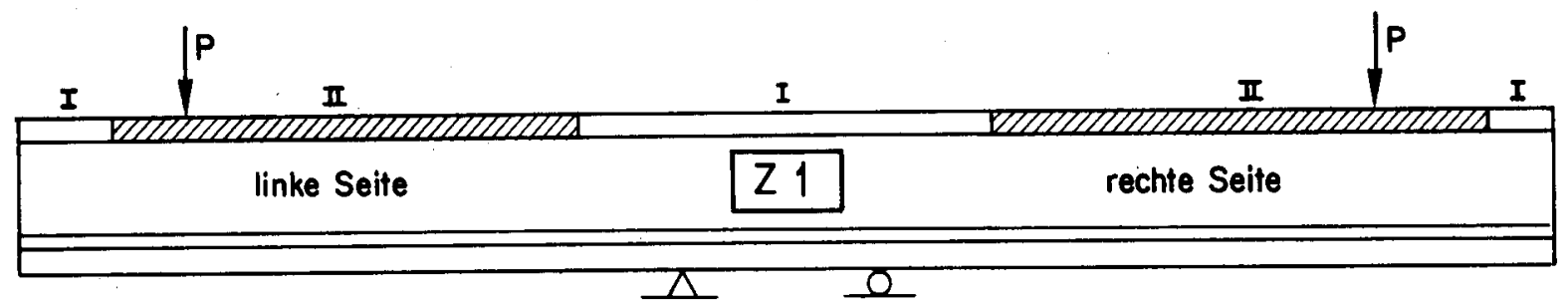

Obere = unfere Loge der Plattenquerarmierung
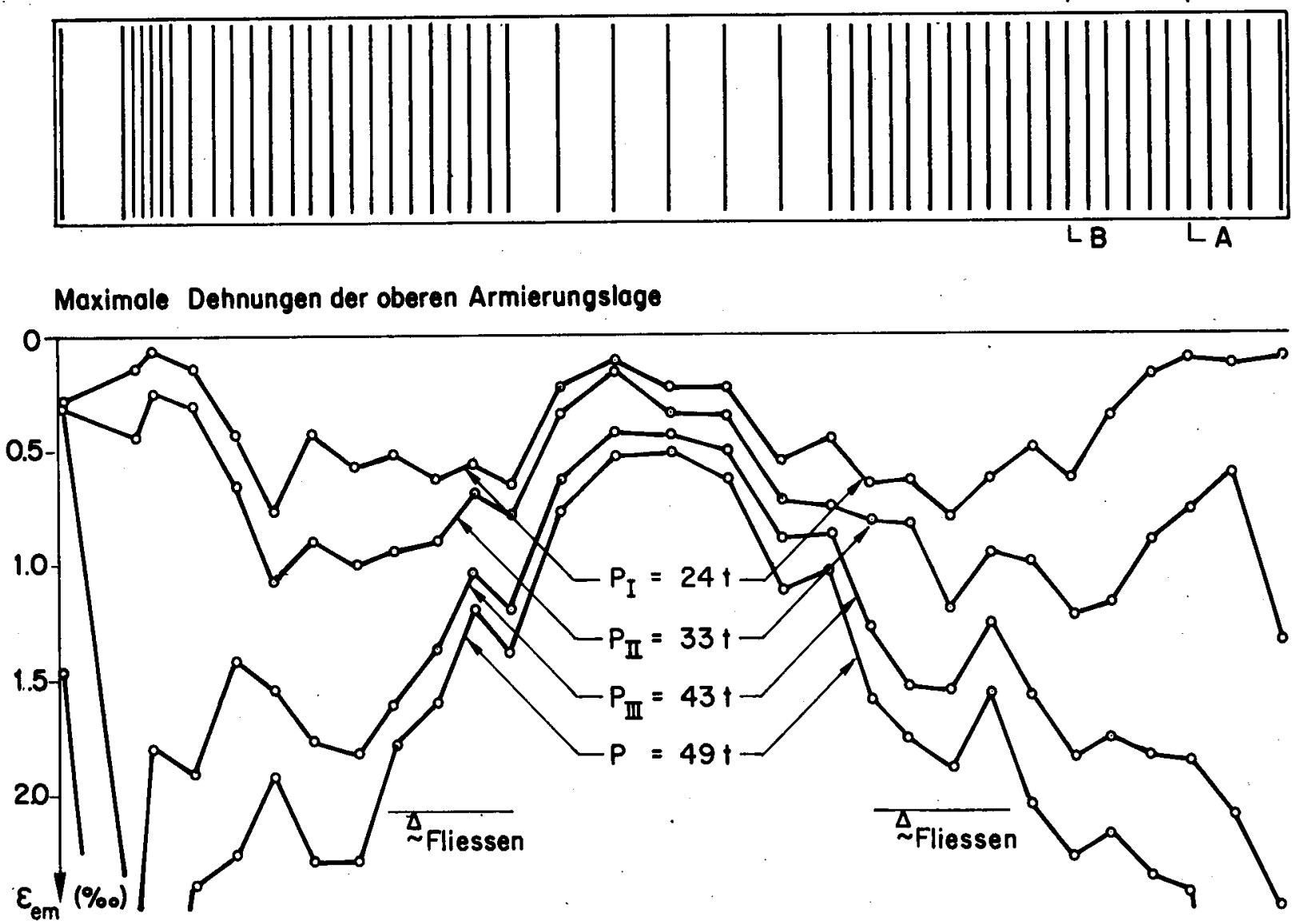

\section{Maximale Dehnungen der unteren Armierungslage}

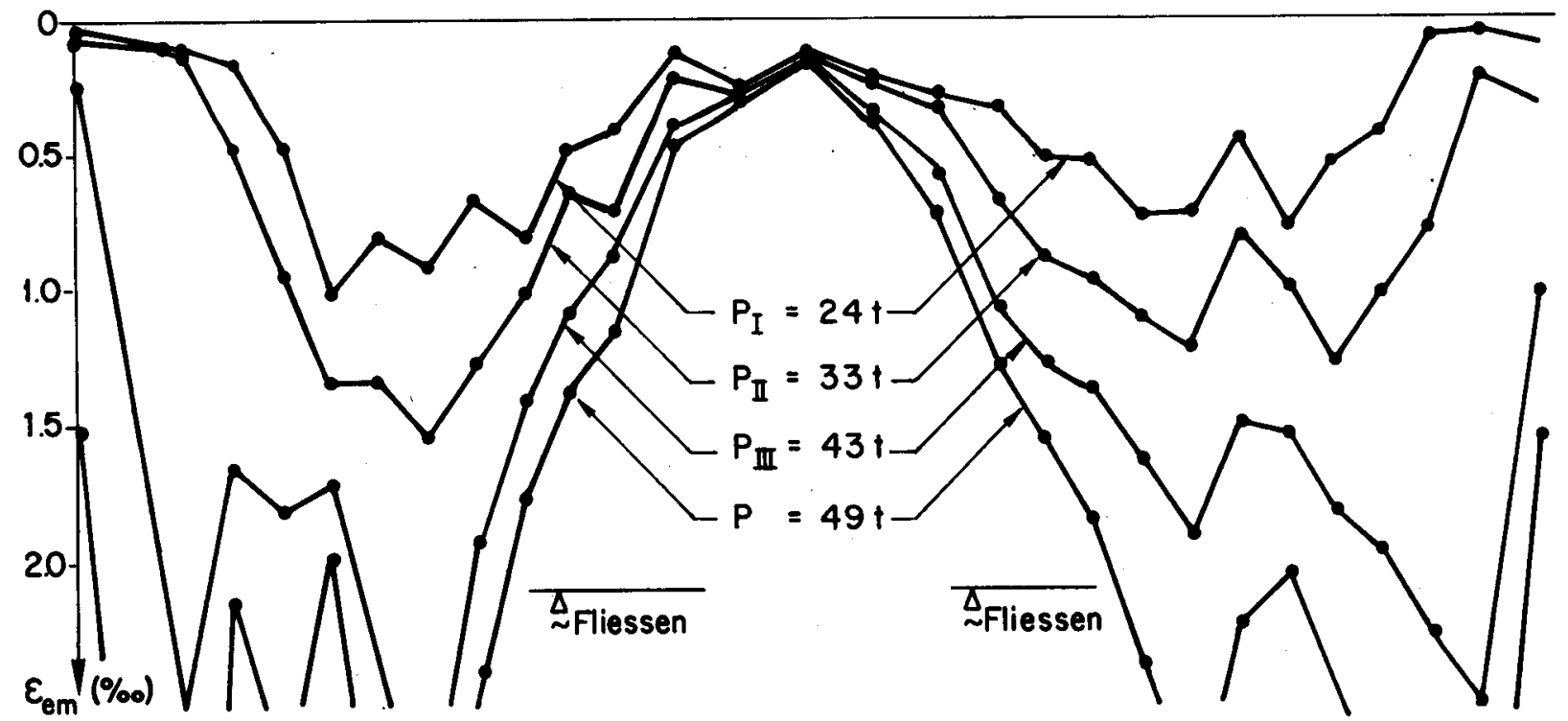

Bild 19-a: Dehnungen der Plattenquerarmierung beim Träger Z1 

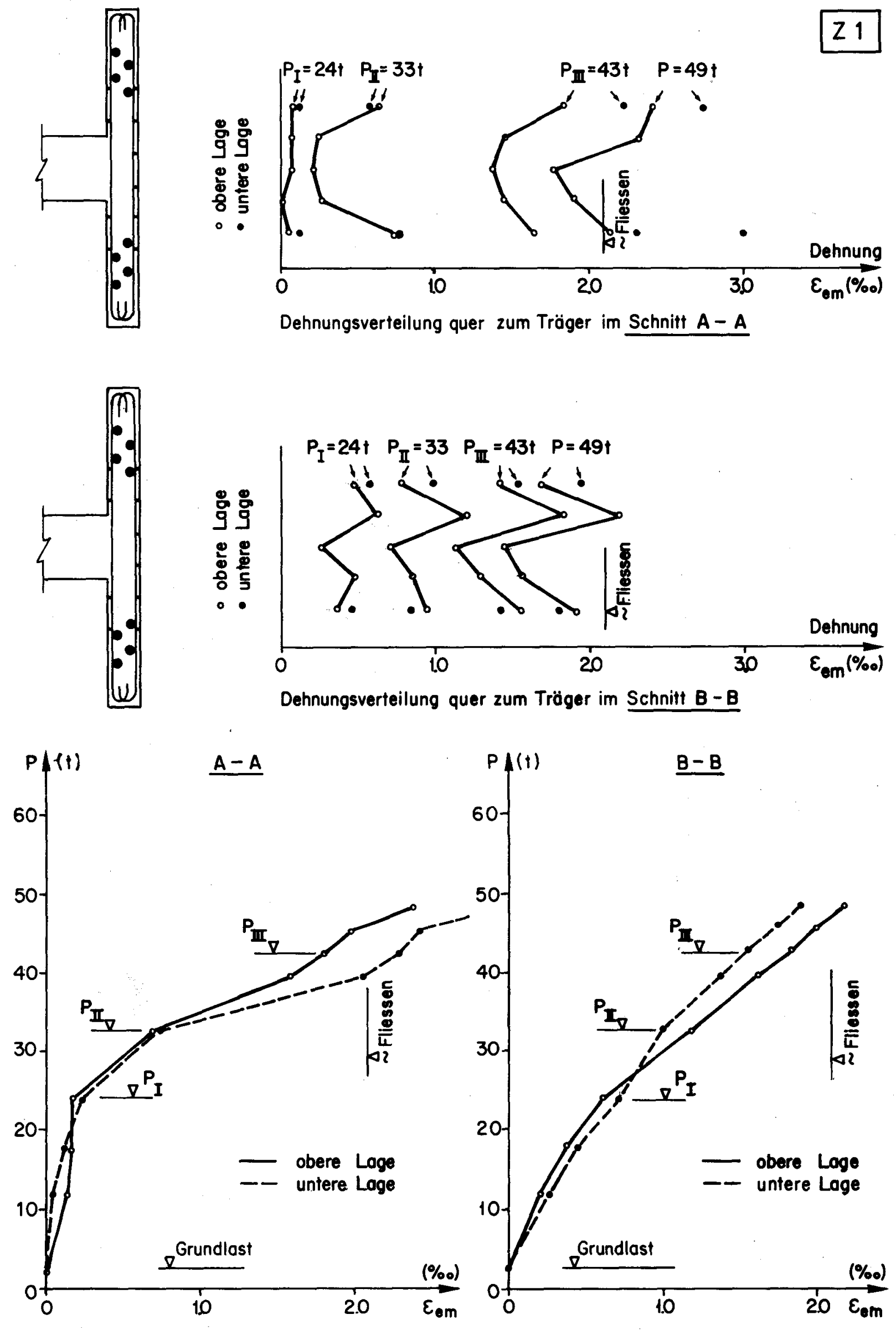

Verlauf der maximalen Dehnungen in den Schnitten A-A und B-B

Bild 19-b : Dehnungen der Plattenquerarmierung beim Träger Z 1 


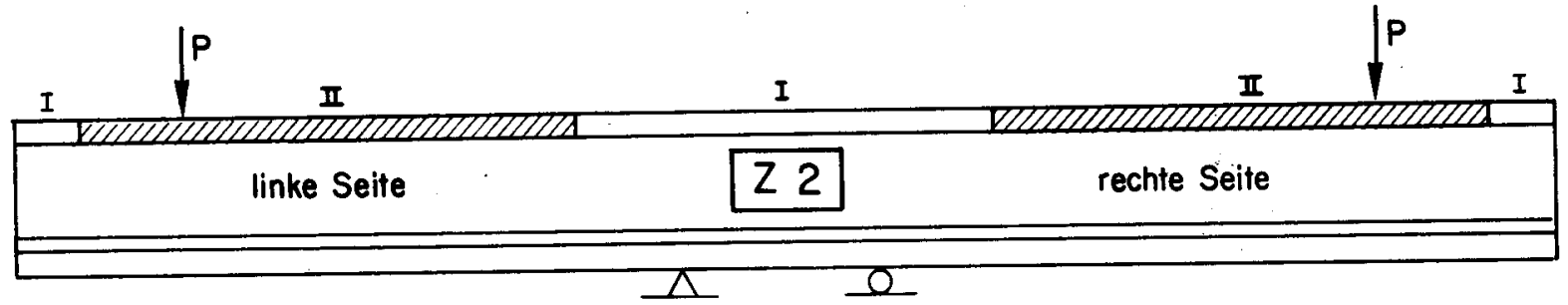

Obere = untere Lage der Plattenquerarmierung

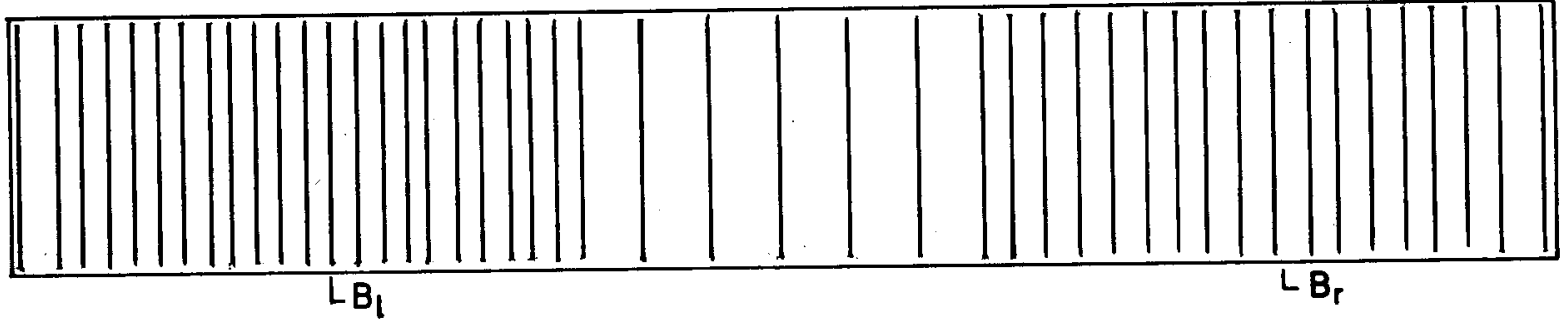

Maximale Dehnungen der oberen Armierungslage

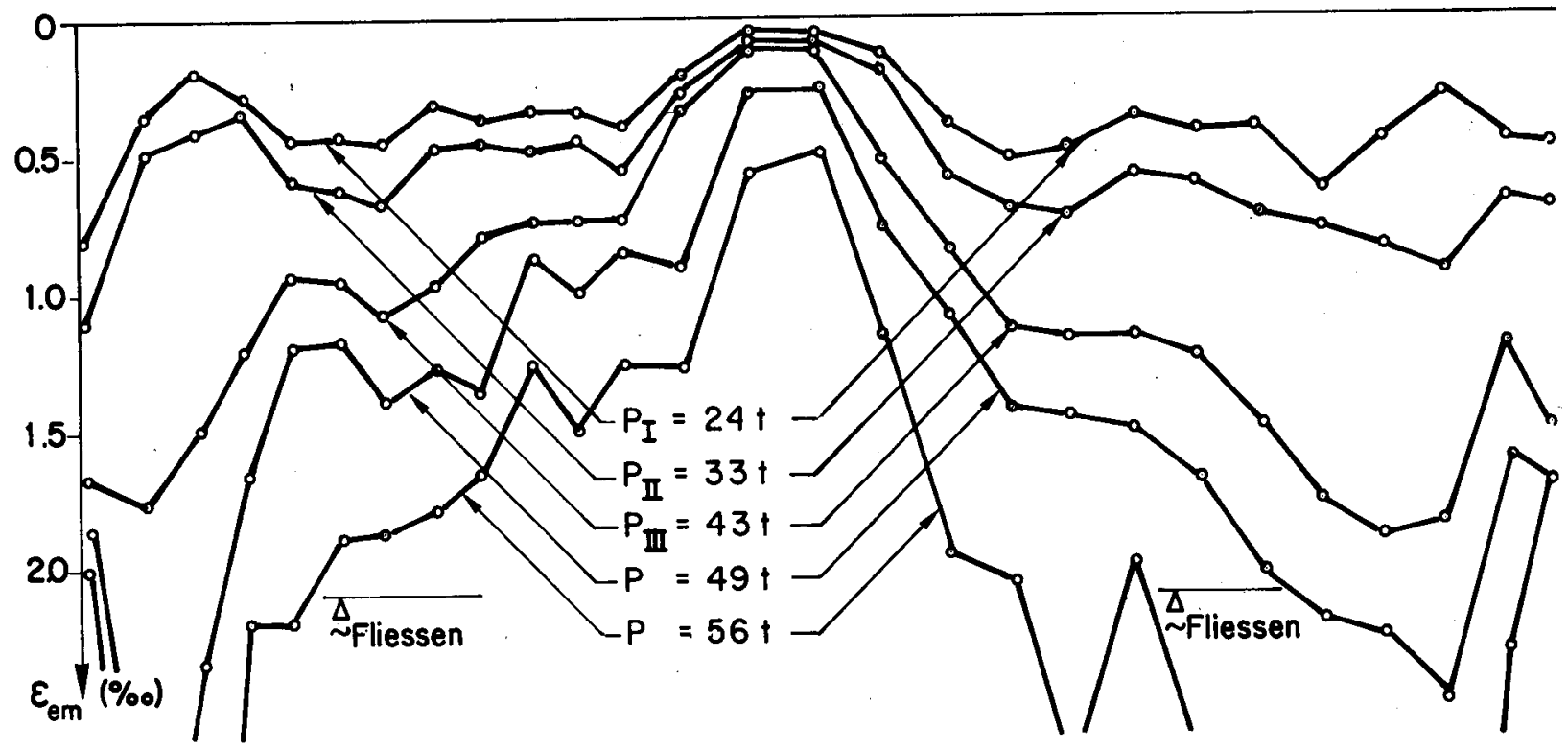

Maximale Dehnungen der unteren Armierungslage

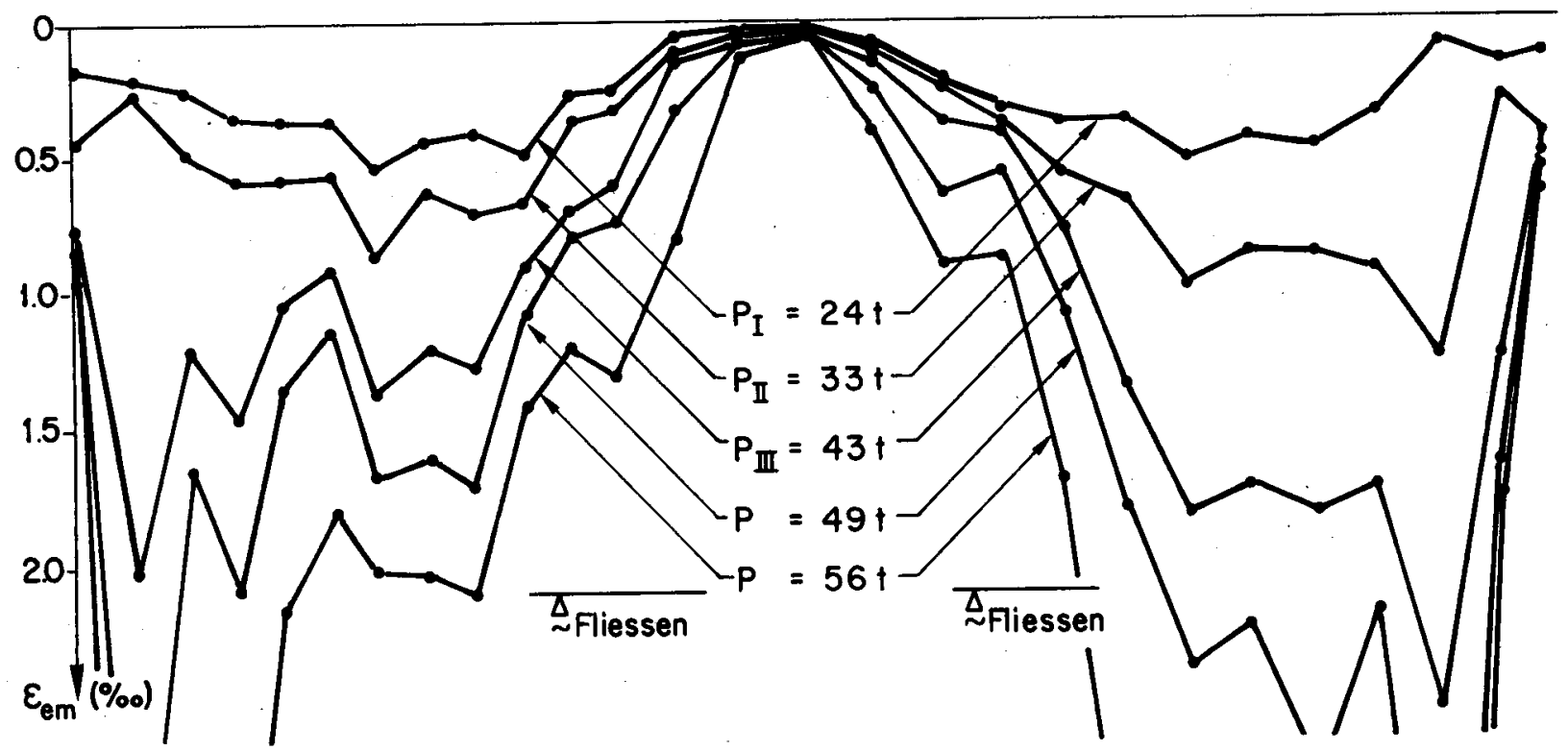

Bild 20-a: Dehnungen der Plattenquerarmierung beim Träger Z 2 

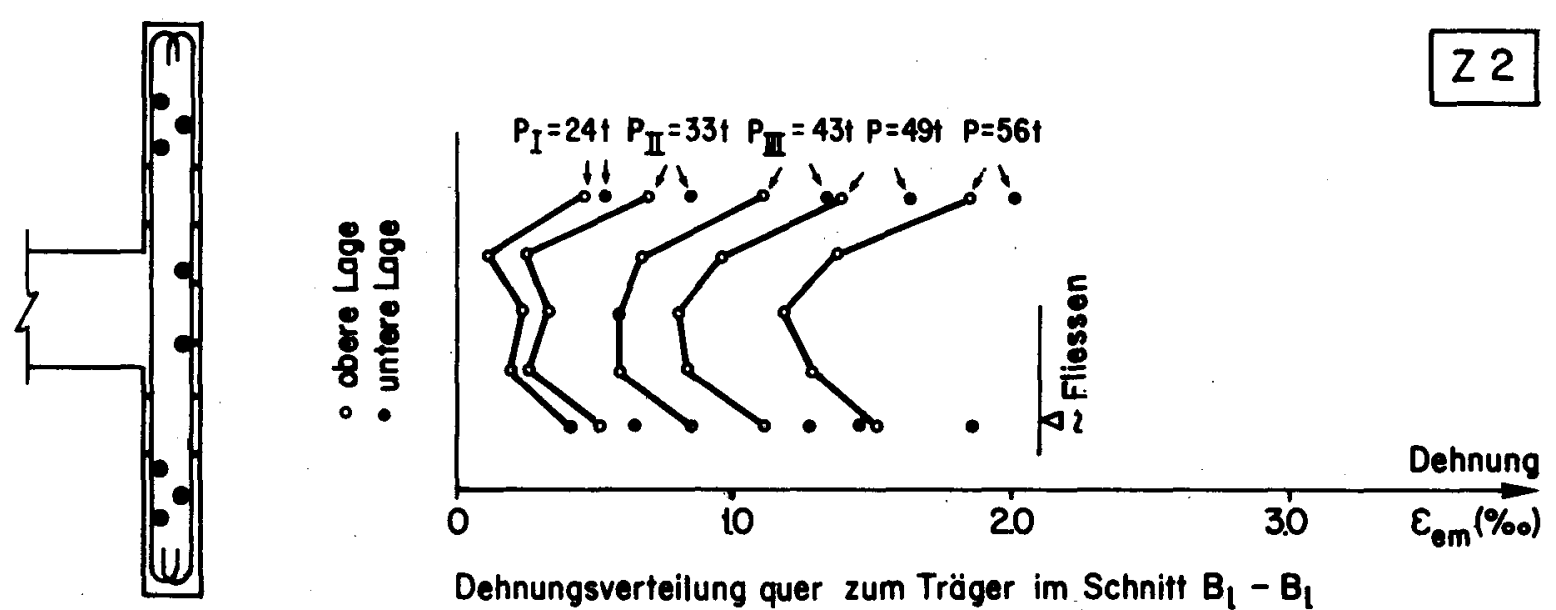

Dehnungsverteilung quer zum Träger im Schnitł $B_{\mathbf{l}}-B_{\mathbf{l}}$
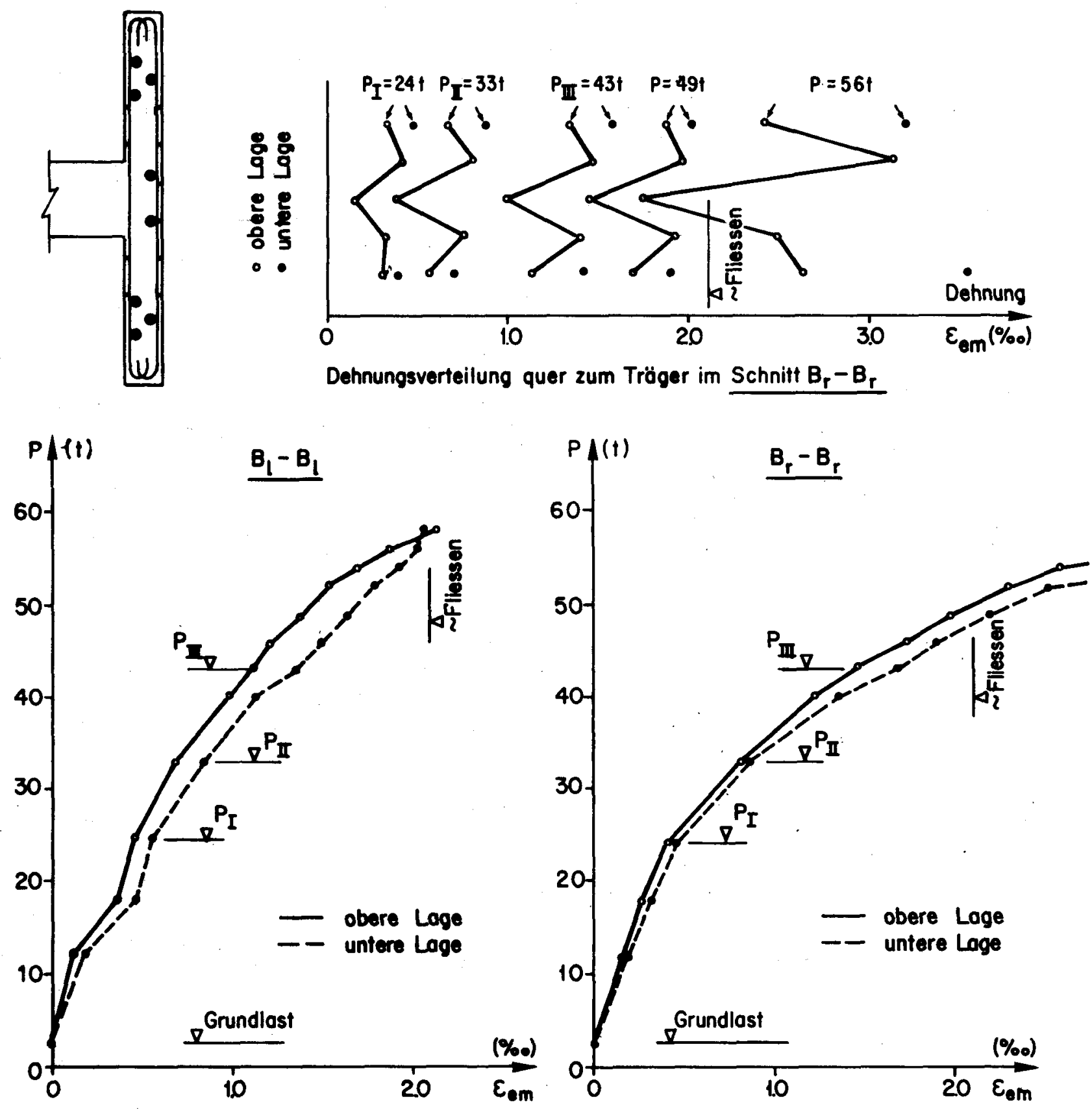

Verlauf der maximalen Dehnungen in den Schnitten $B_{1}-B_{1}$ und $B_{r}-B_{r}$

Bild 20-b : Dehnungen der Plattenquerarmierung beim Träger $\mathrm{Z} 2$ 


\begin{tabular}{|l|l|l|l|}
\hline III & II & II & II \\
\hline linke Seite: schloff armiert & I 4 & rechte Seite teilweise vorgespannt \\
\hline
\end{tabular}

0

Obere Loge der Plattenquerarmierung

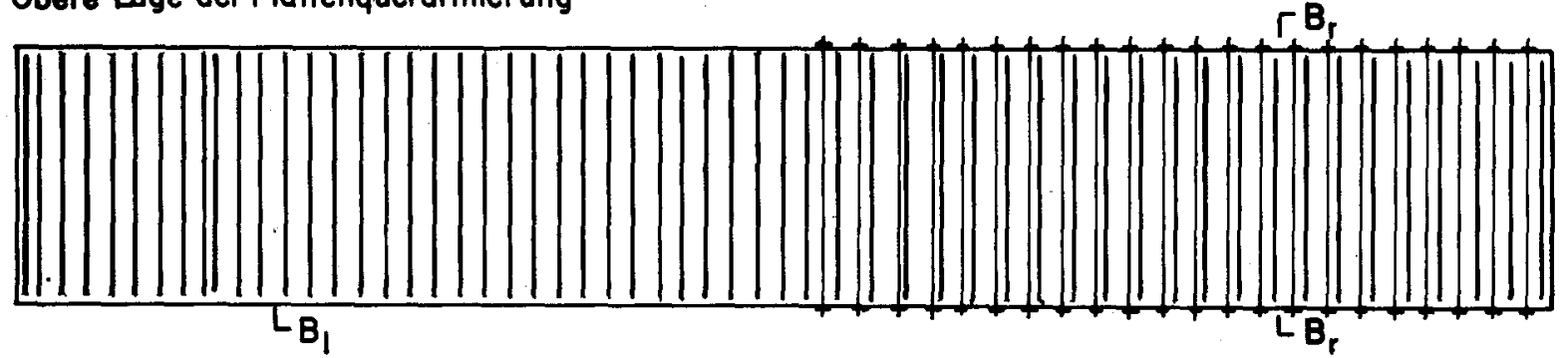

Untere Lage der Plattenquerarmierung

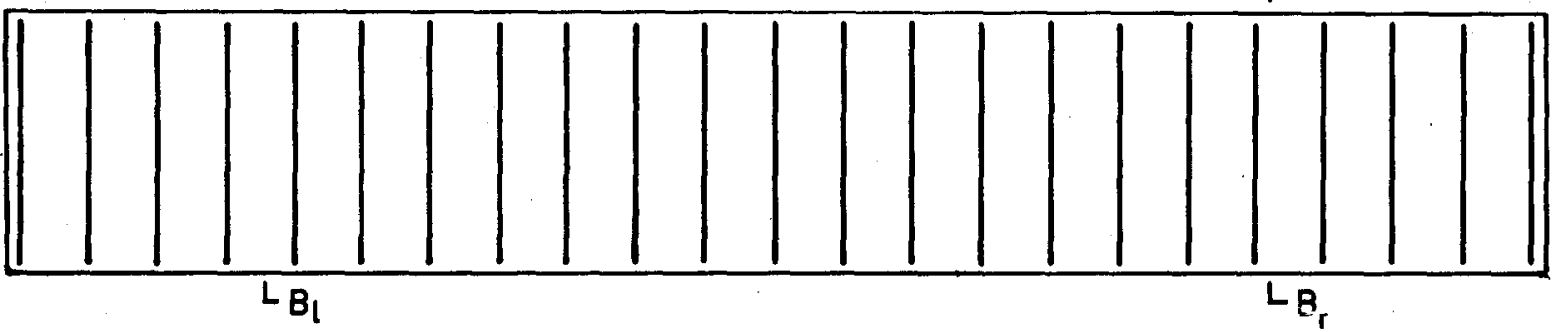

Maximale Dehnungen der oberen Armierungslage

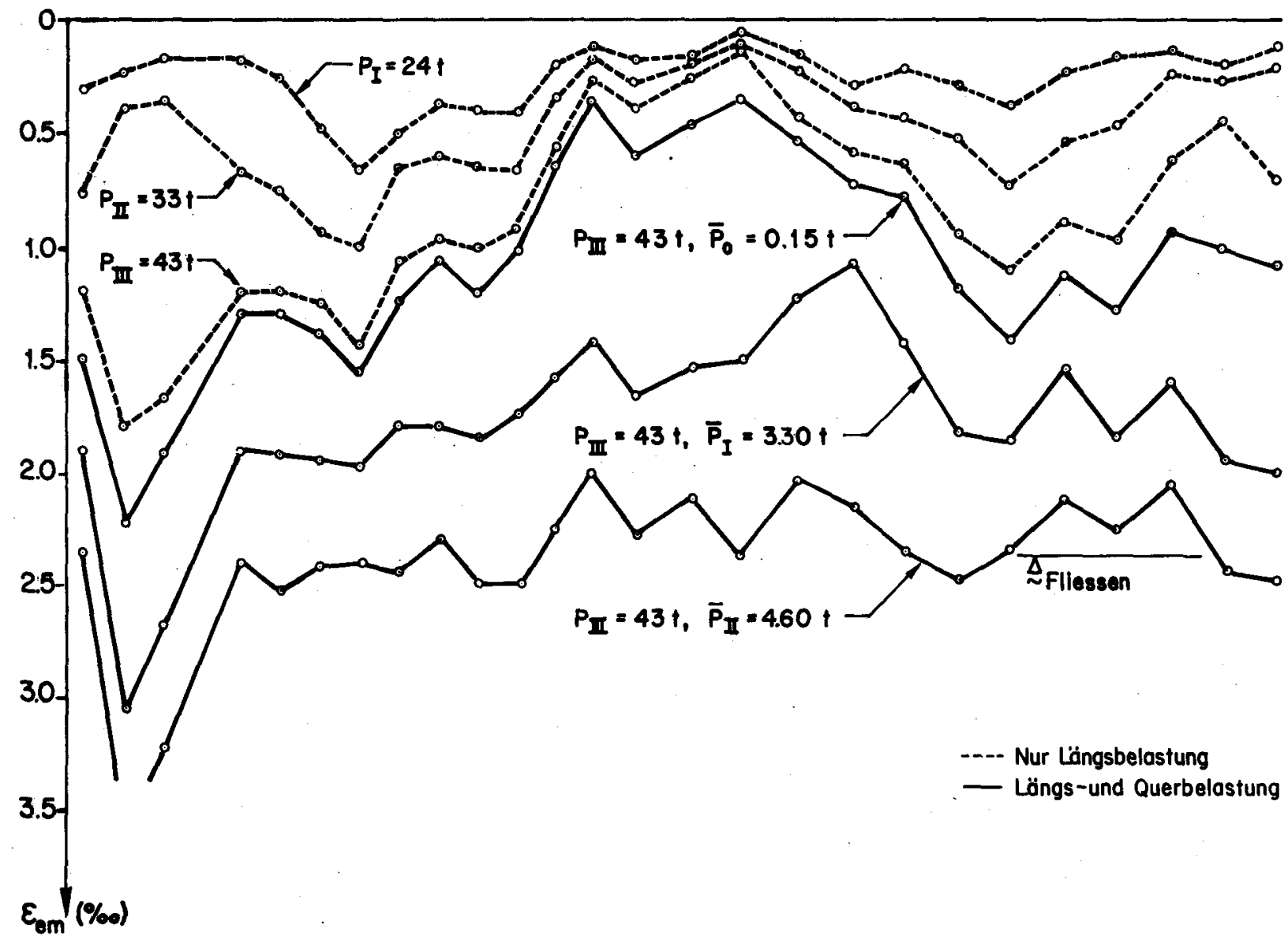

Bild 21-a: Dehnungen der Plattenquerarmierung beim Träger Z4 

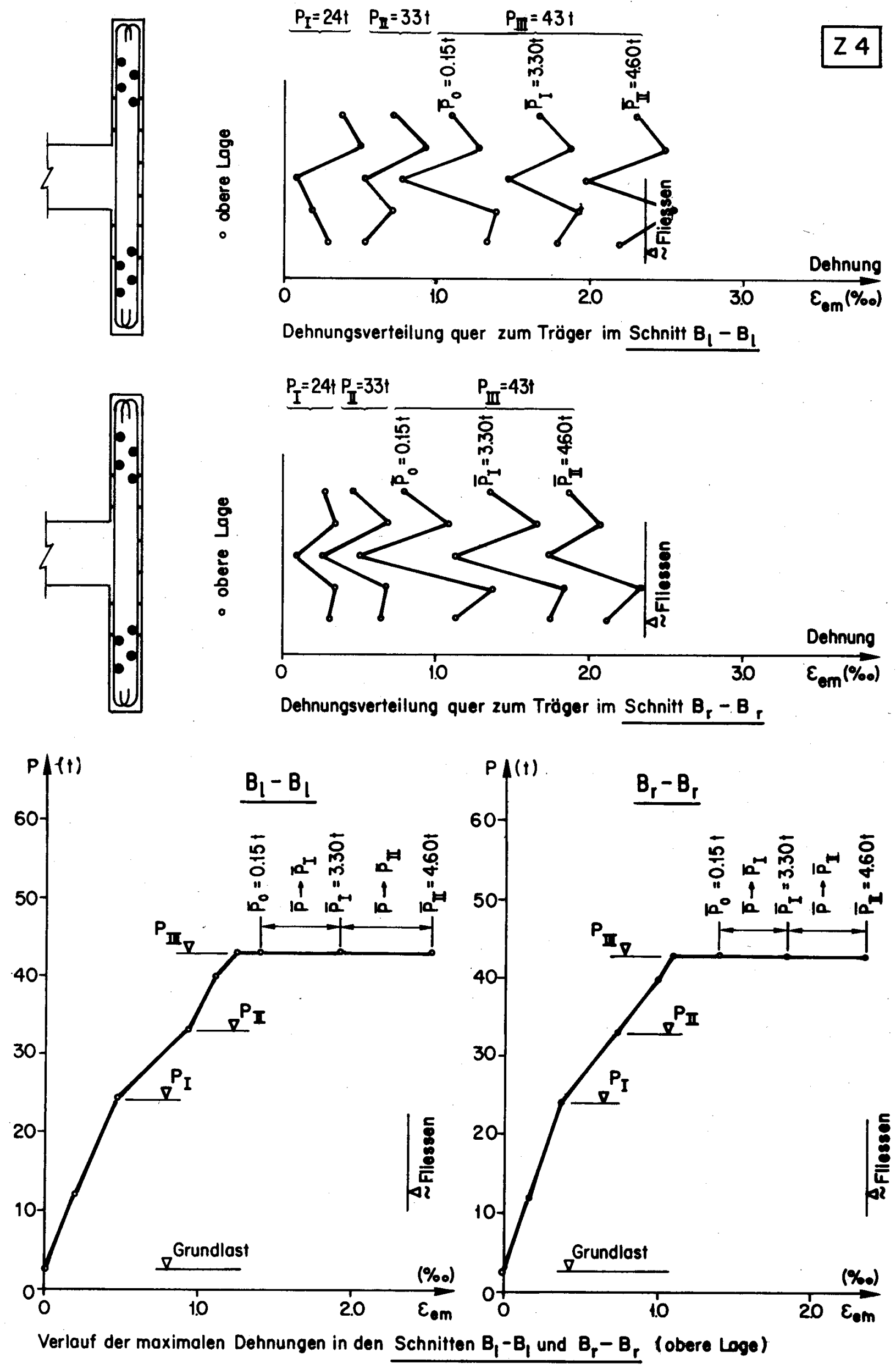

Bild 21-b : Dehnungen der Plattenquerarmierung beim Träger $Z 4$ 

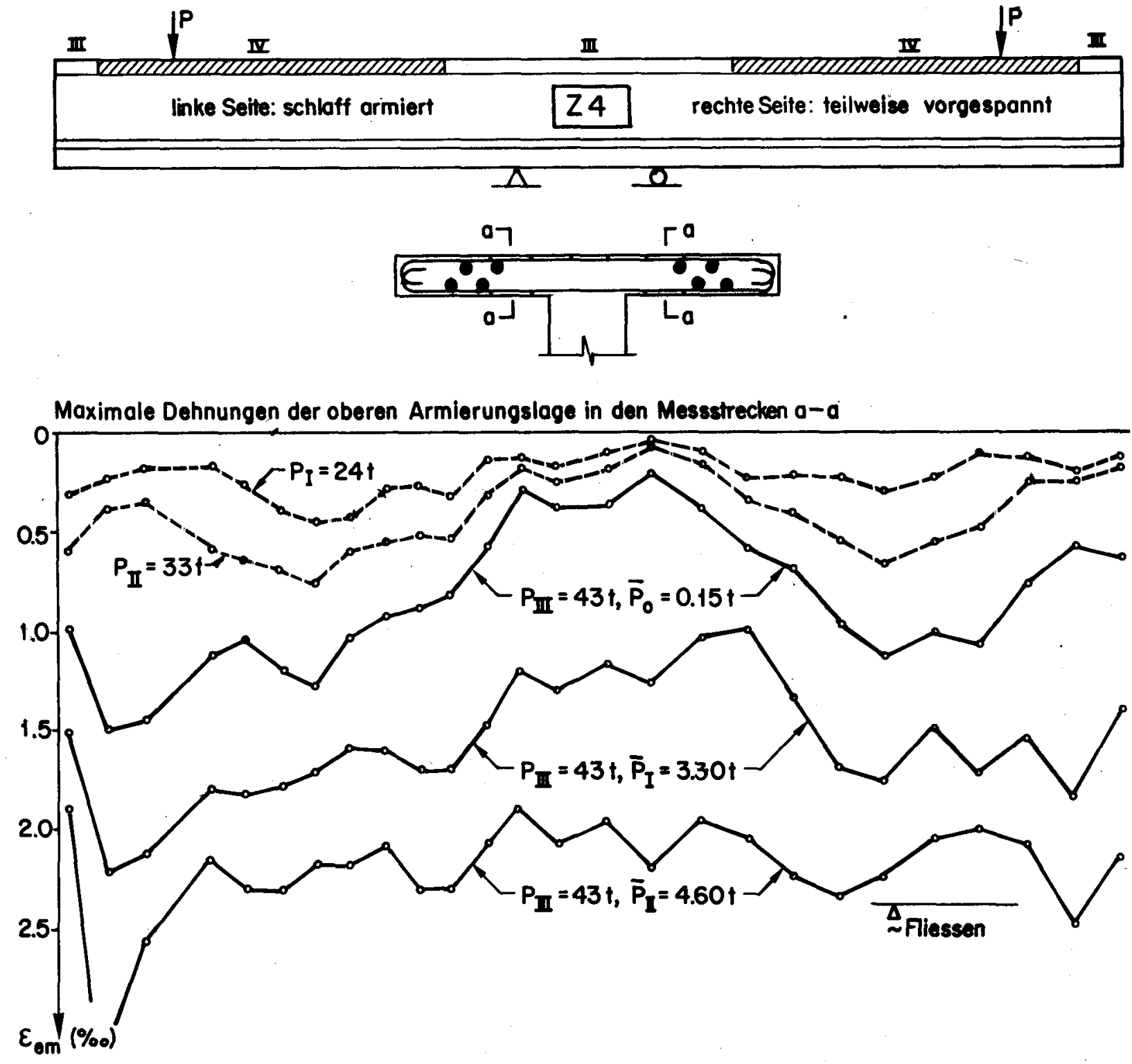

Maximale Dehnungen der unteren Armierungslage in den Messstrecken a-a

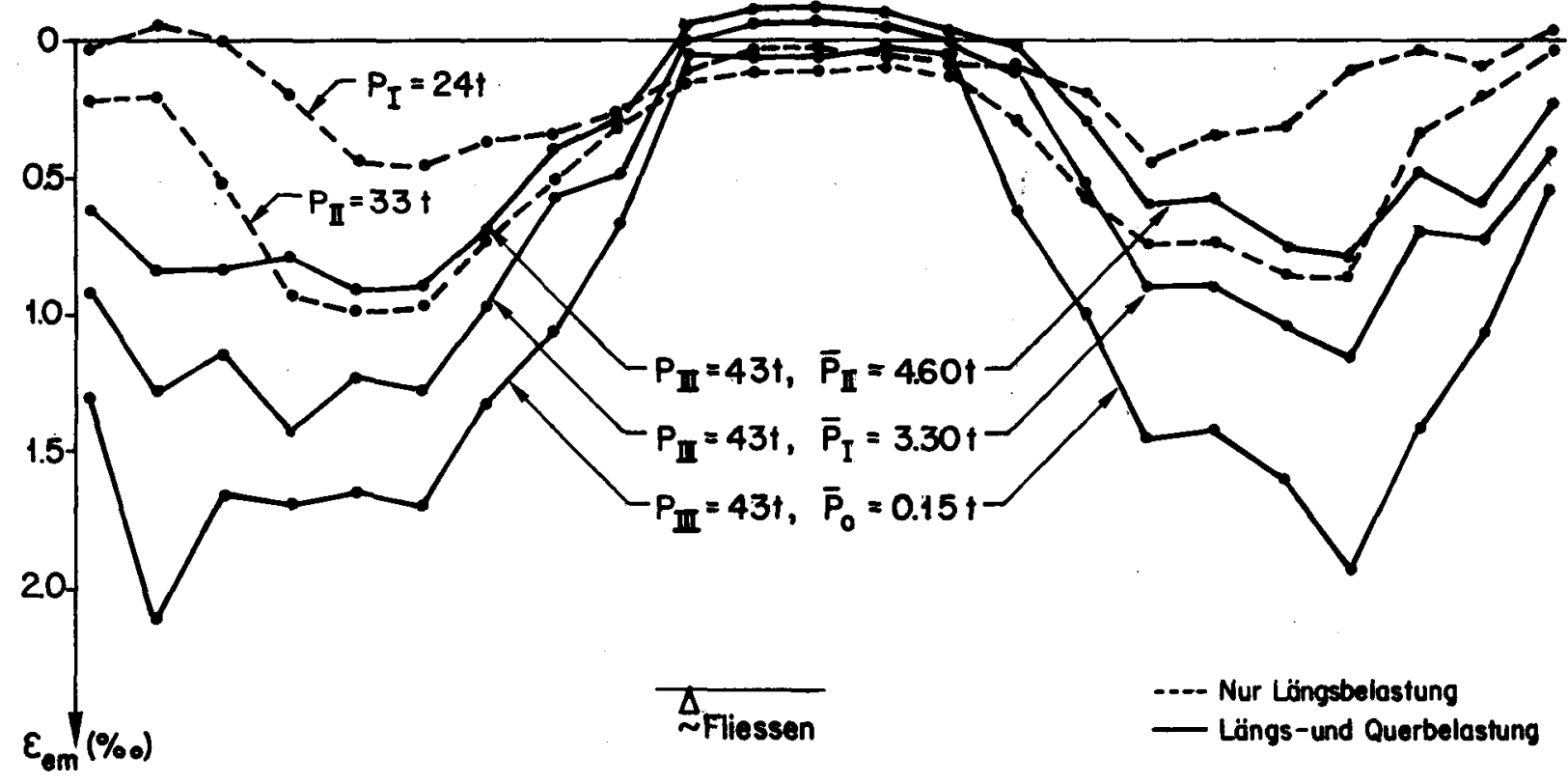

Bild 21-c: Dehnungen der Plattenquerarmierung beim Träger Z 4 


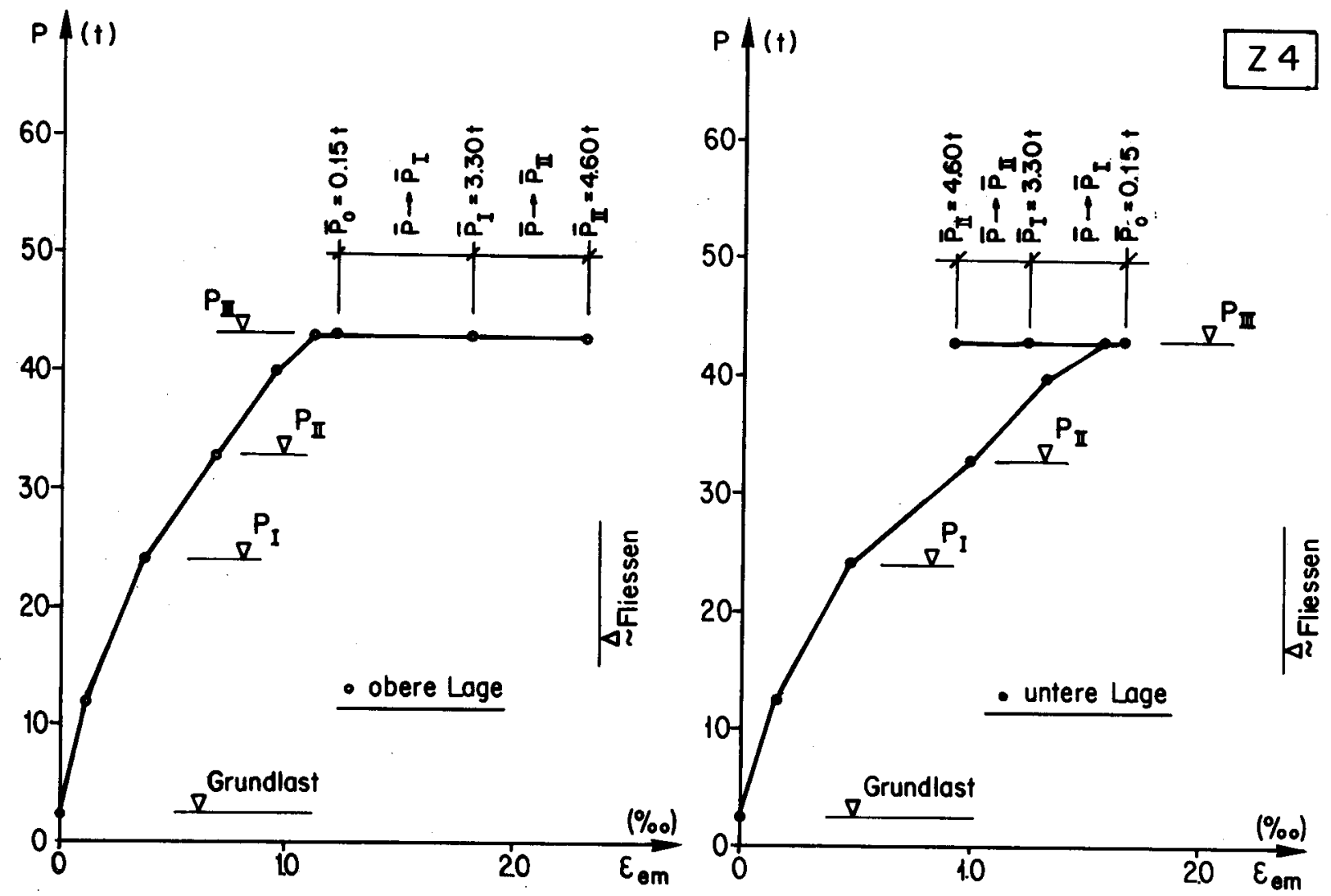

Verlauf der max. Dehnungen der Messstrecken $a-a$ im Schnitf $B_{1}-B_{1}$
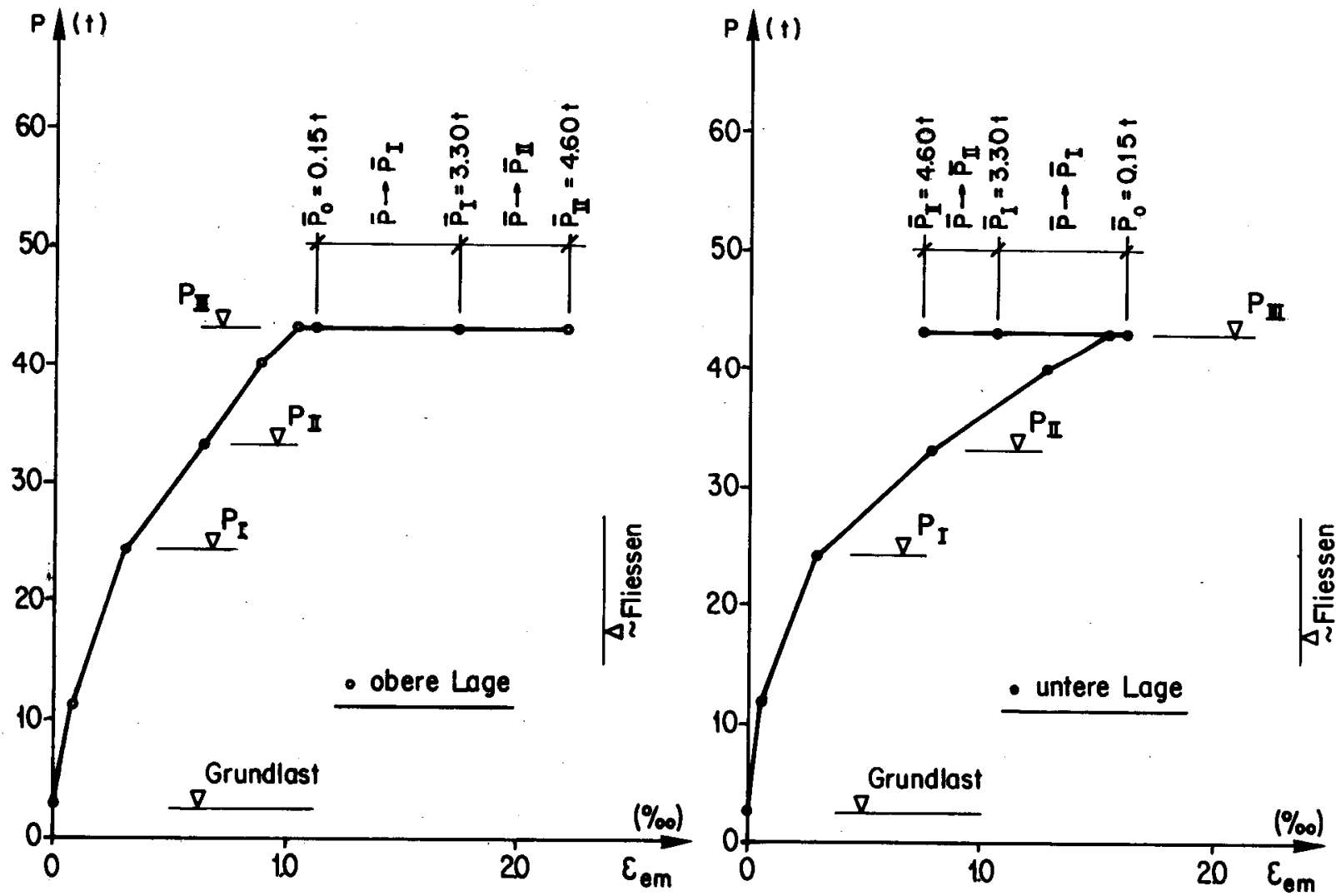

Verlauf der max. Dehnungen der Messstrecken $0-0$ im Schnitt $B_{r}-B_{r}$

Bild 21-d: Dehnungen der Plattenquerarmierung beim Träger Z 4 


\begin{tabular}{|l|l|l|l|l|}
\hline II & III & II & III \\
\hline linke Seite: schlaff armiert & $Z 3$ & rechte Seife: teilweise vorgesponnt \\
\hline
\end{tabular}
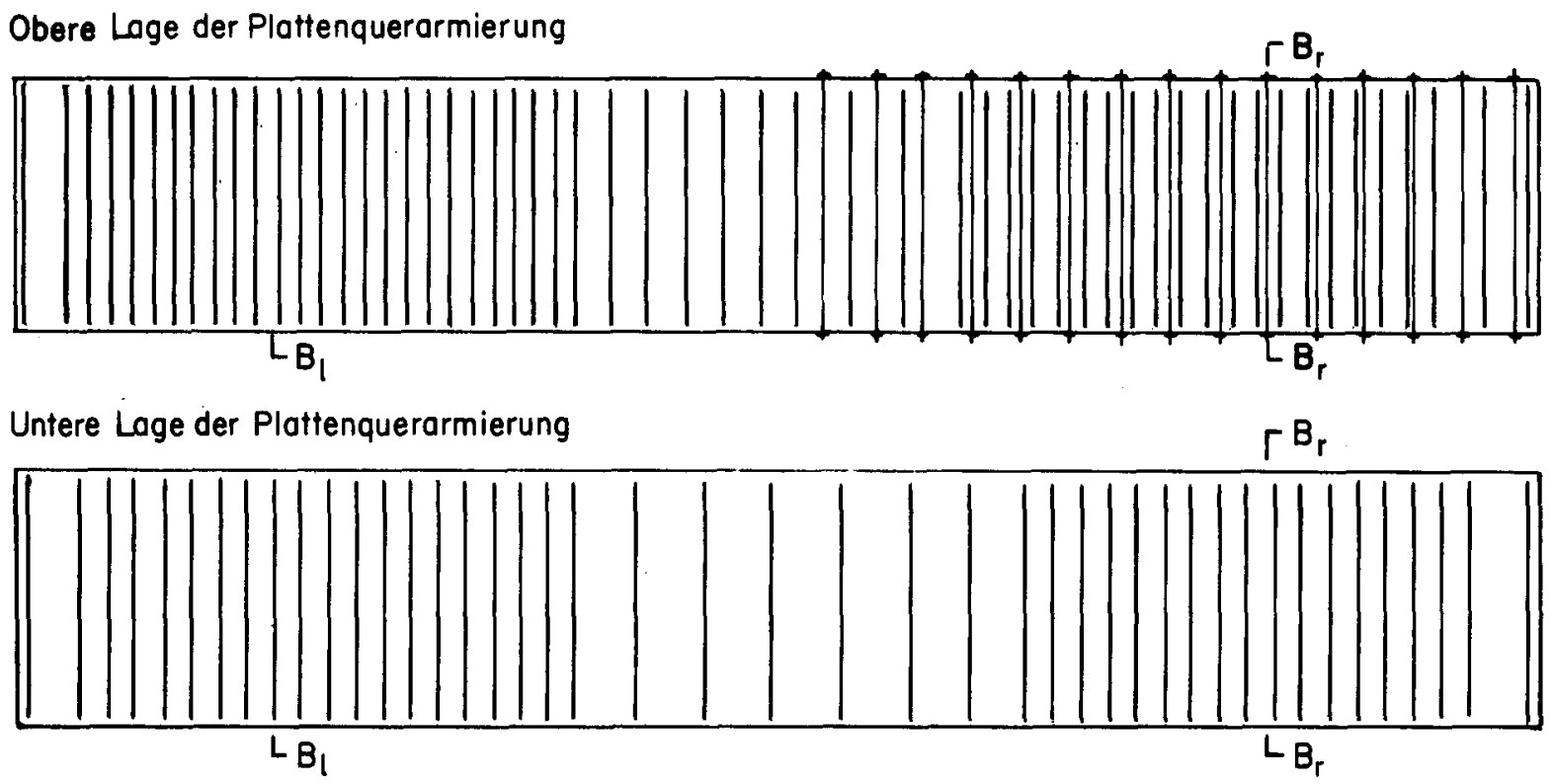

Maximale Dehnungen der oberen Armierungslage

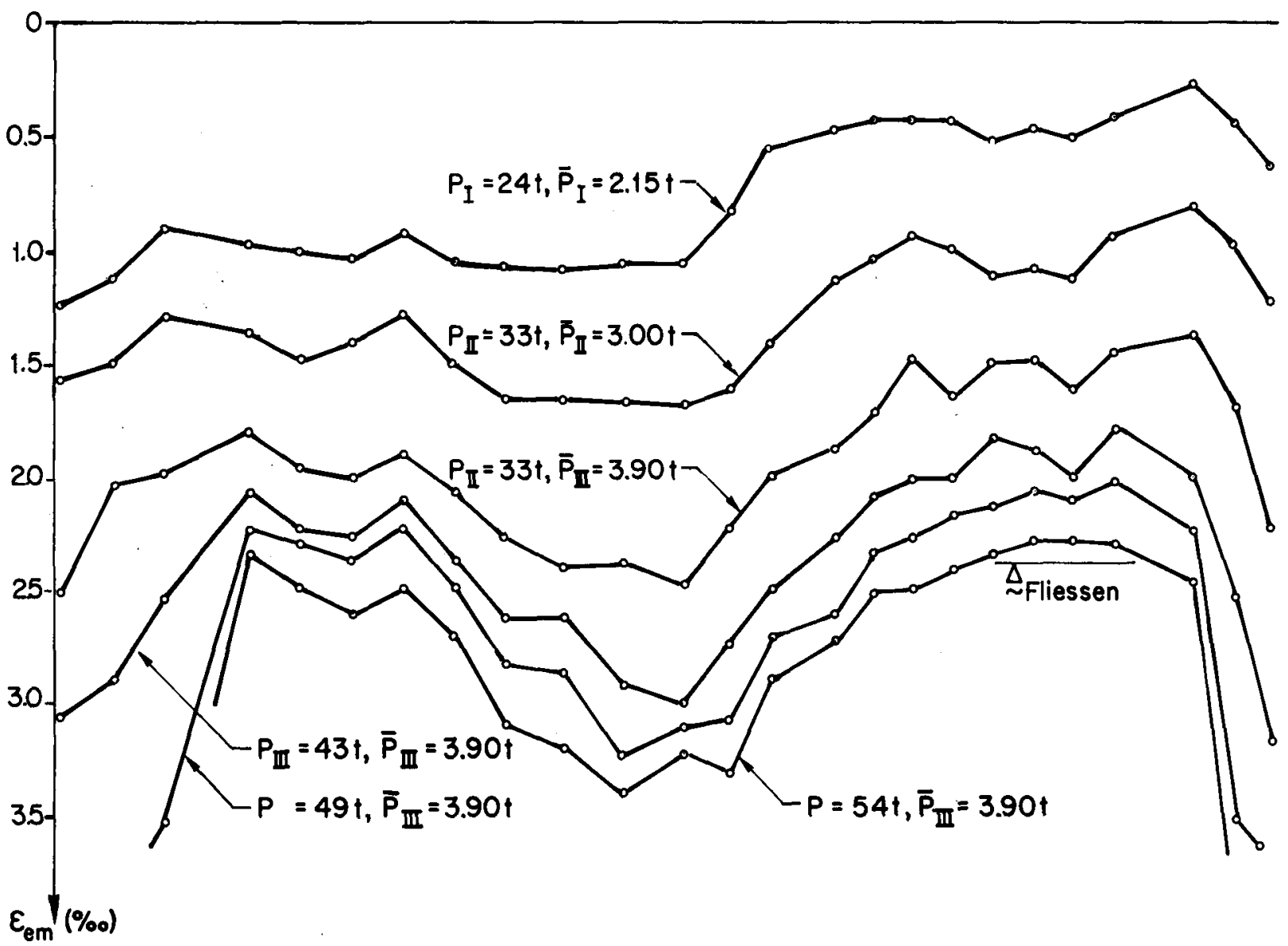

Bild 22- $a$ : Dehnungen der Plattenquerarmierung beim Träger $Z 3$ 

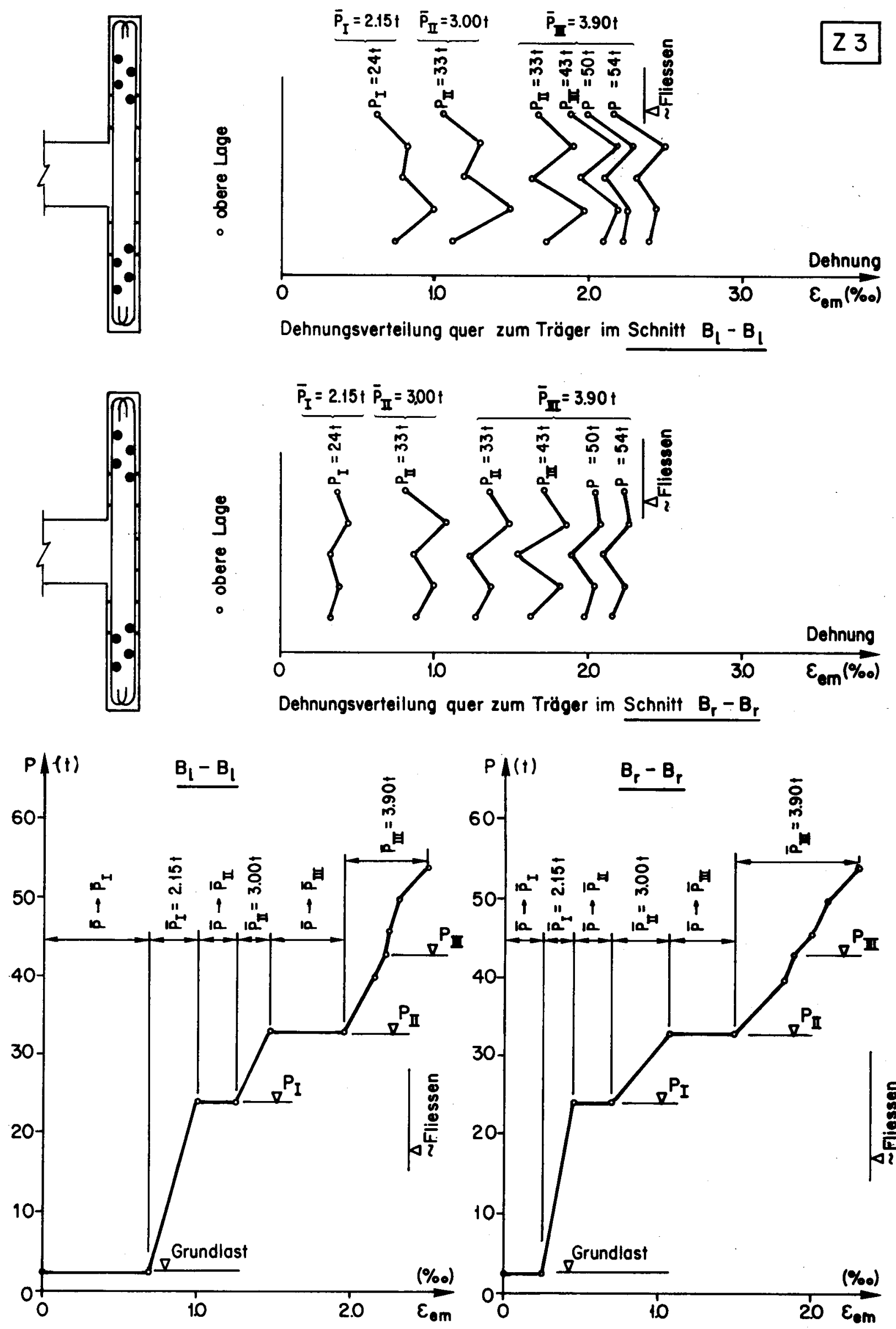

Verlauf der maximalen Dehnungen in den Schnitten $B_{1}-B_{1}$ und $B_{r}-B_{r}$ (obere Loge)

Bild 22-b : Dehnungen der Plattenquerarmierung beim Träger Z 3 

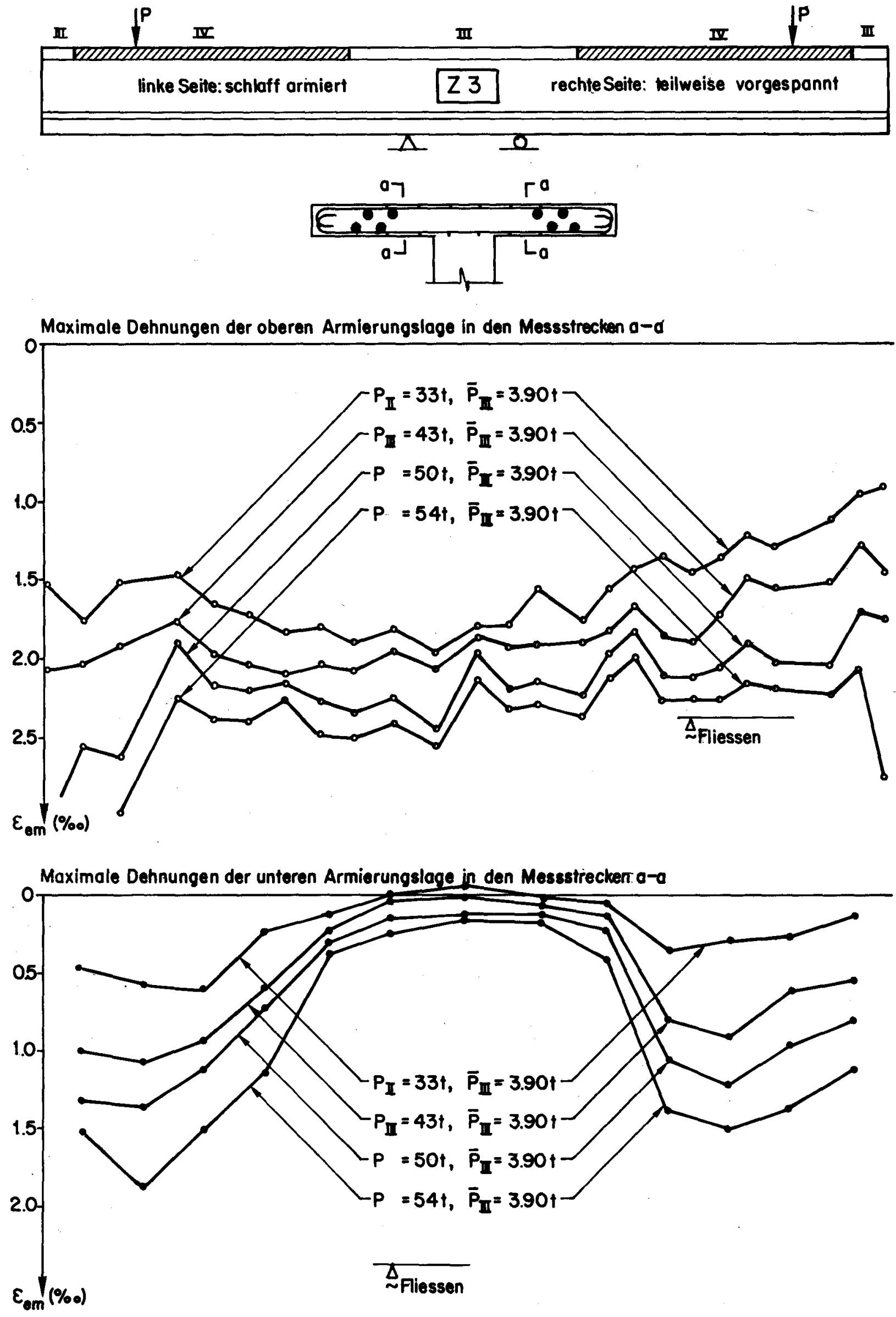

Bild 22-c; Dehnungen der Plattenquerarmierung beim Träger Z 3 

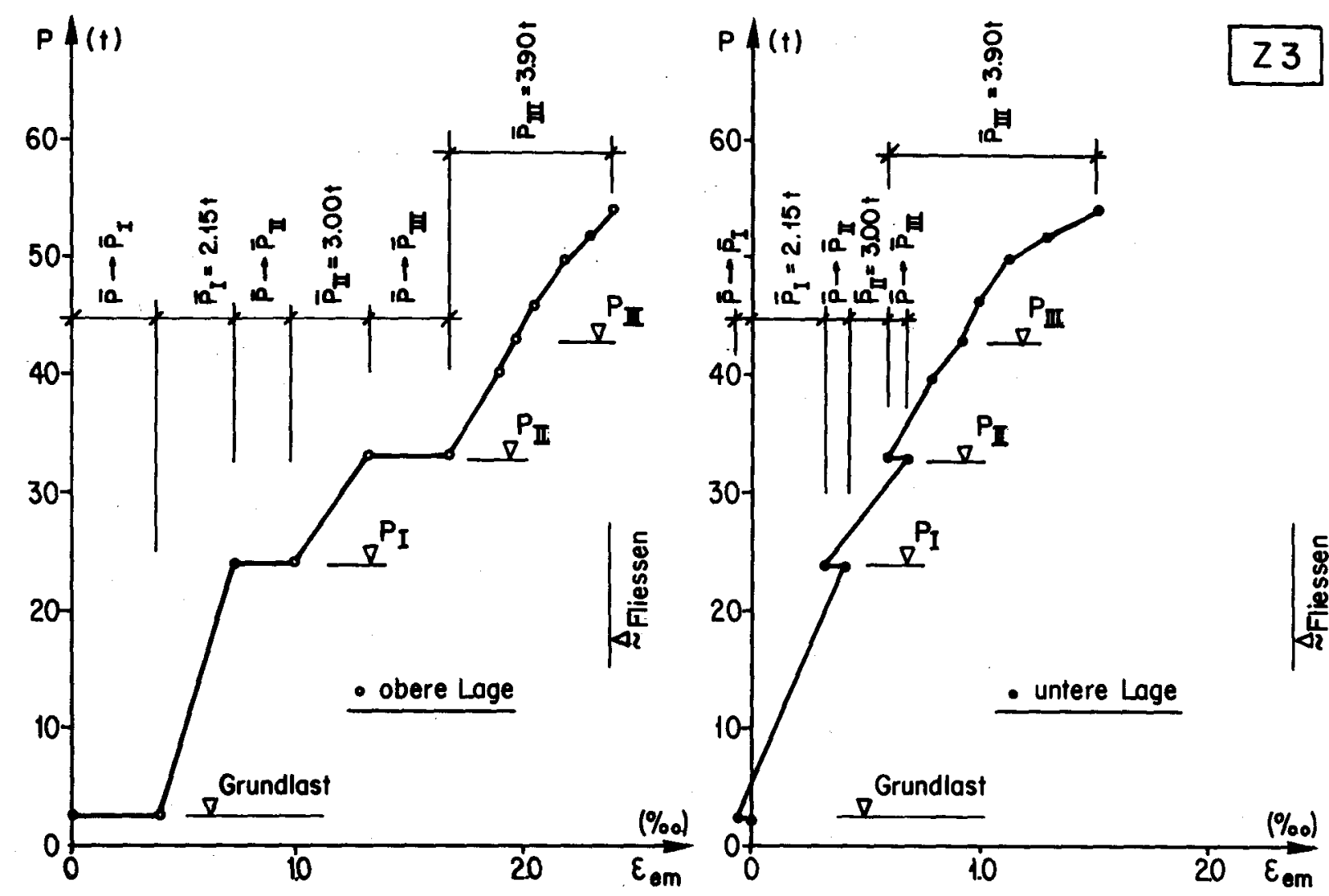

Verlauf der max. Dehnungen der Messstrecken a-a im Schnitt $B_{l}-B_{l}$
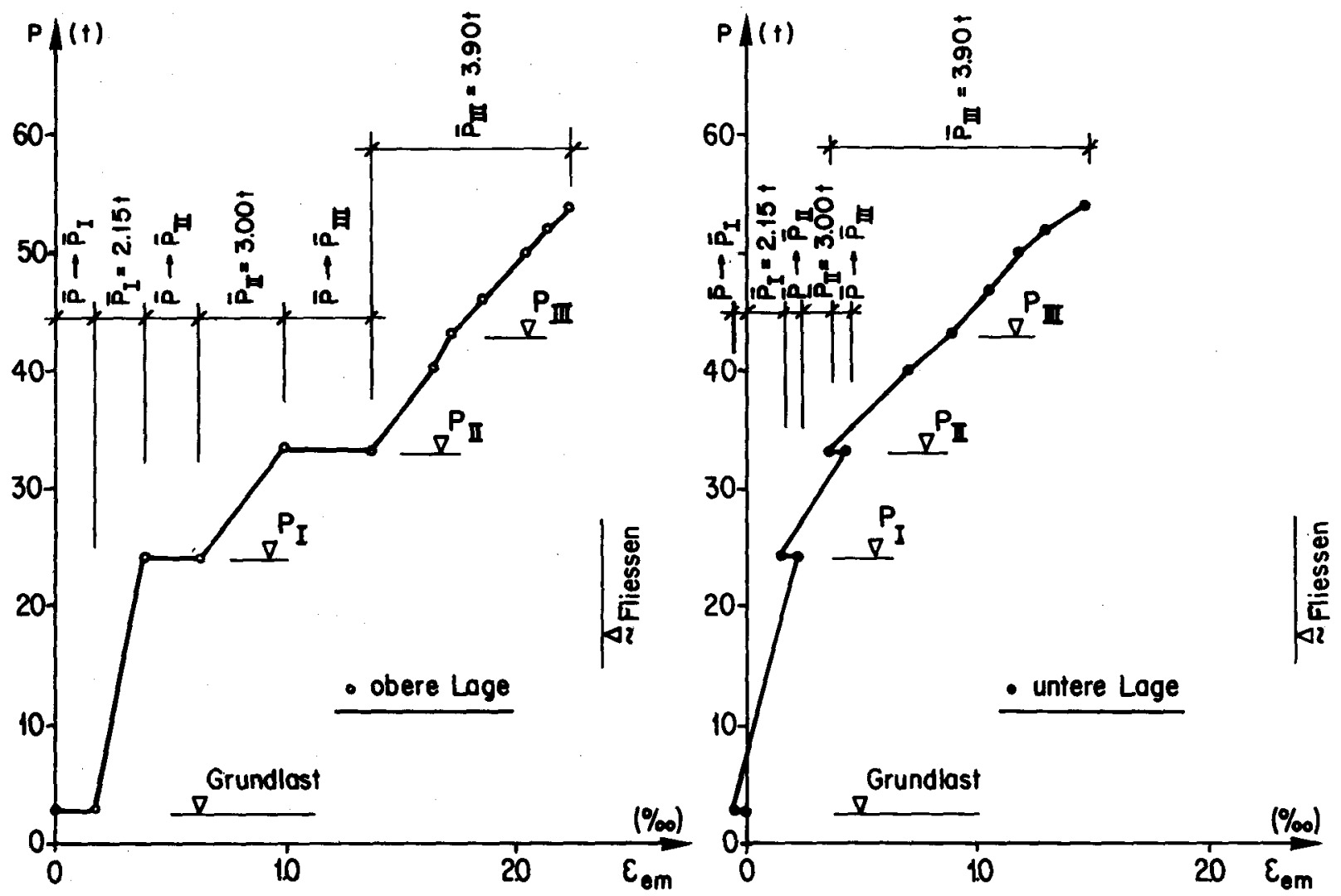

Verlauf der max. Dehnungen der Messistrecken $a-0$ im Schnitt $B_{r}-B_{r}$

Bild 22-d: Dehnungen der Plattenquerarmierung beim Träger $Z 3$ 


\begin{tabular}{|l|l|l|l|}
\hline III & III & II \\
\hline linke Seife: schlaff armiert & $\mathrm{Z}$ & rechte Seite: teilweise vorgespannt \\
\hline
\end{tabular}
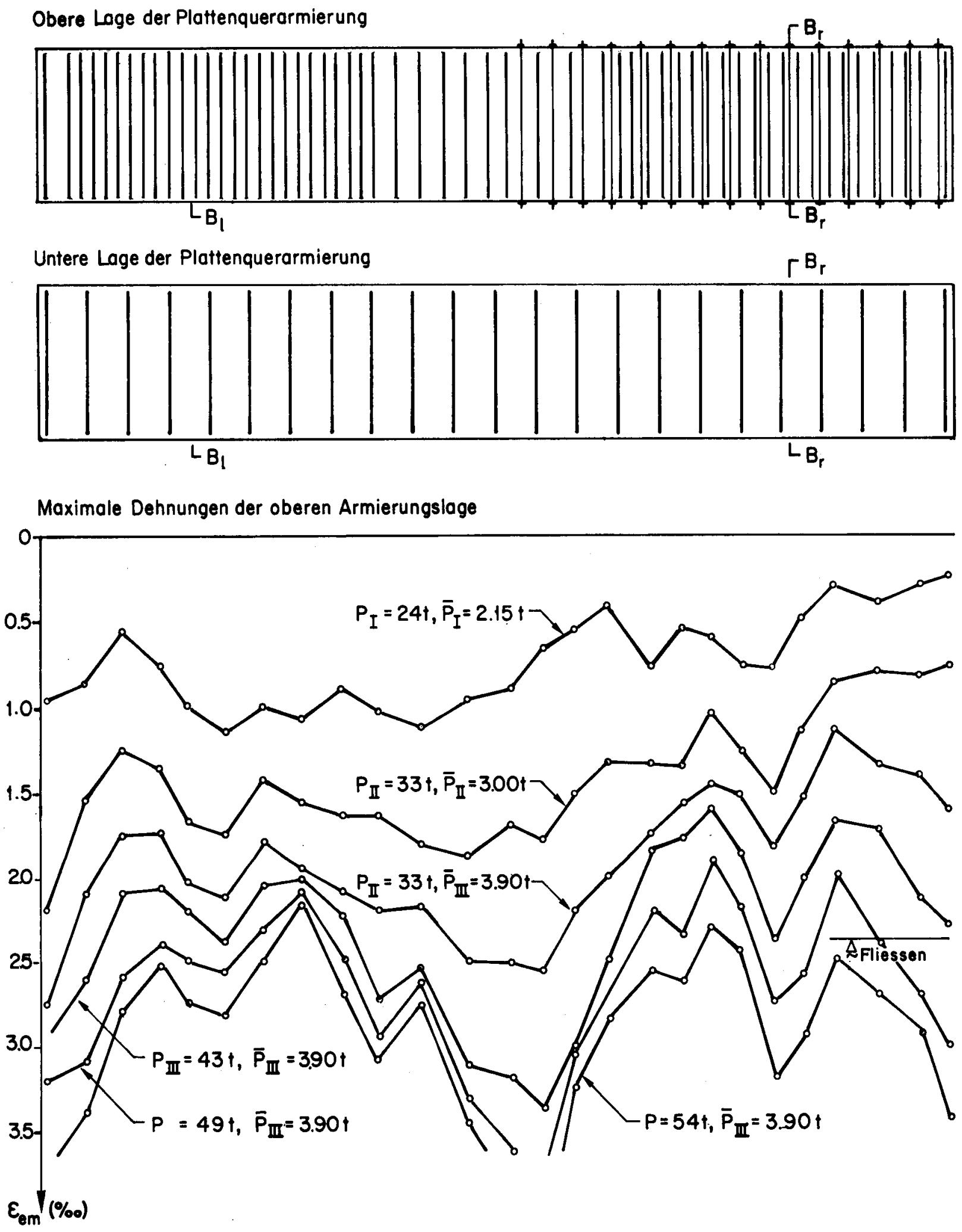

Bild 23-a: Dehnungen der Plattenquerarmierung beim Träger Z 5 

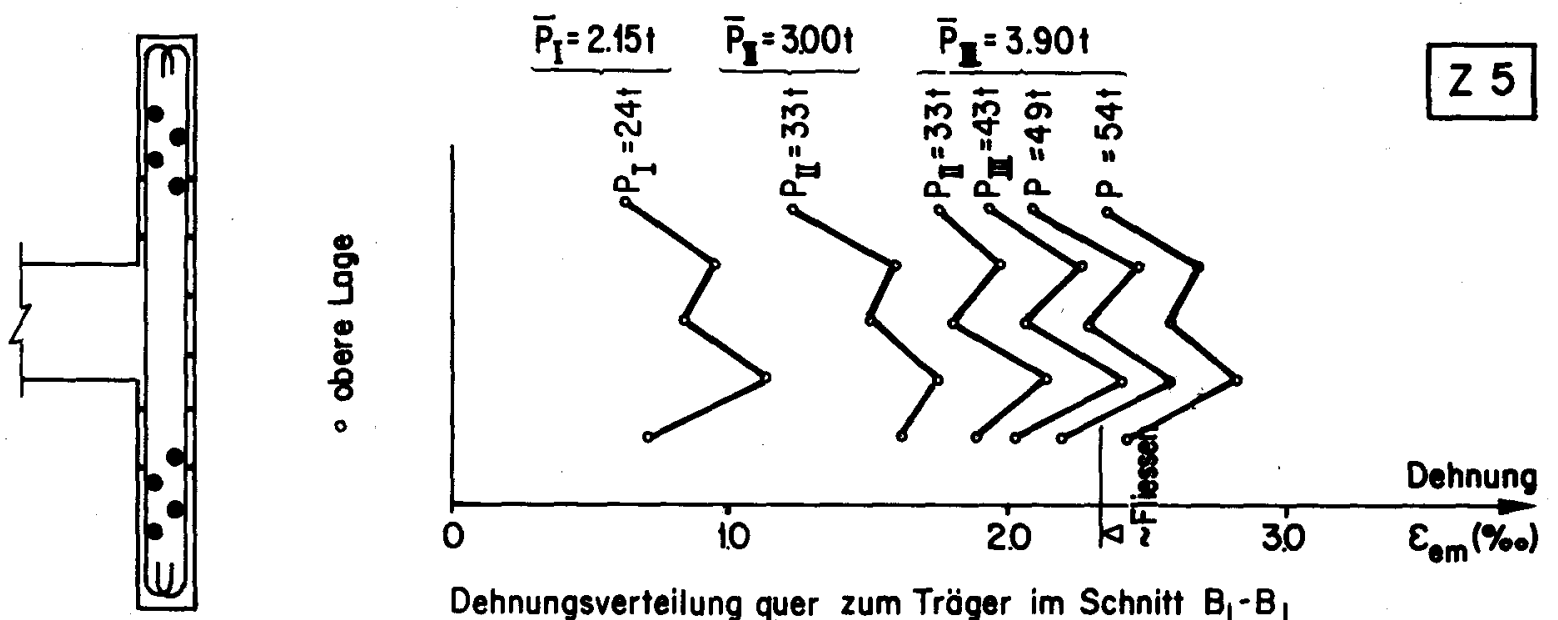

Dehnungsverteilung quer zum Träger im Schnitt $B_{l}-B_{l}$
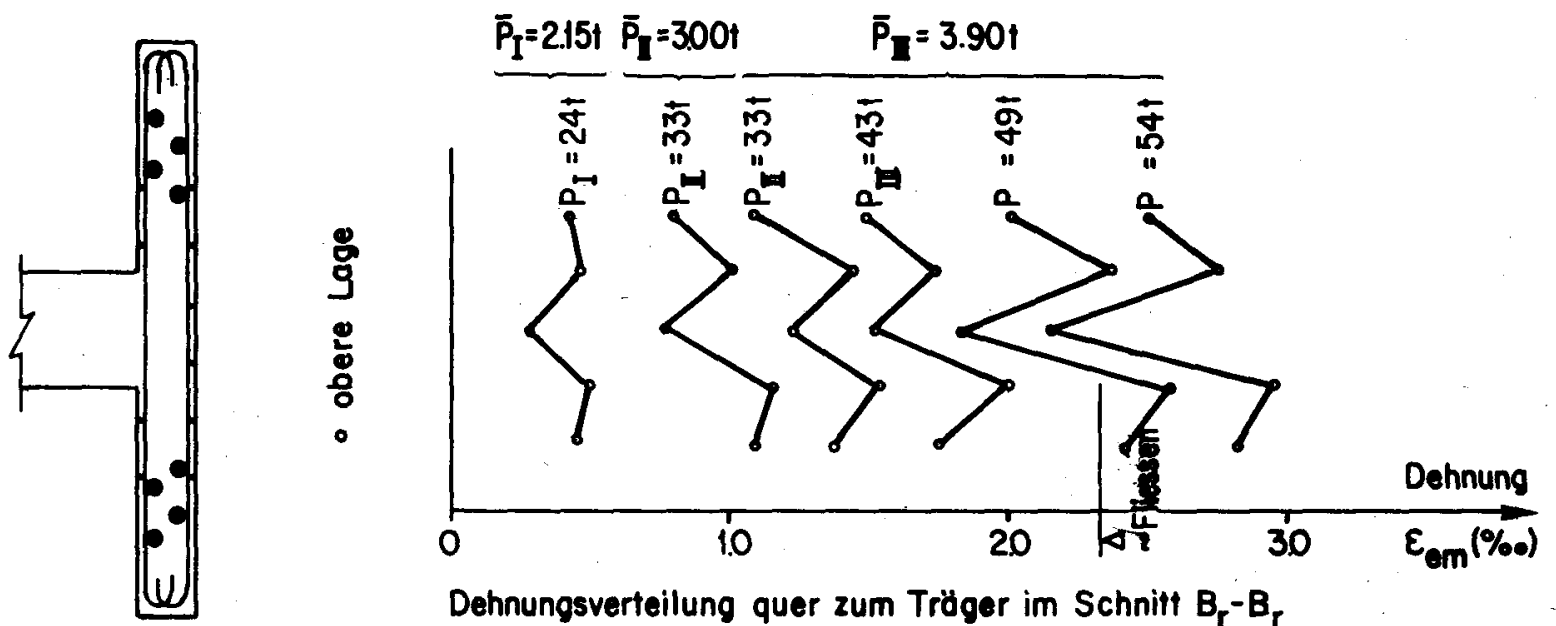

Dehnungsverteilung quer zum Träger im Schnitt $\mathrm{B}_{\mathrm{r}}-\mathrm{B}_{\mathrm{r}}$

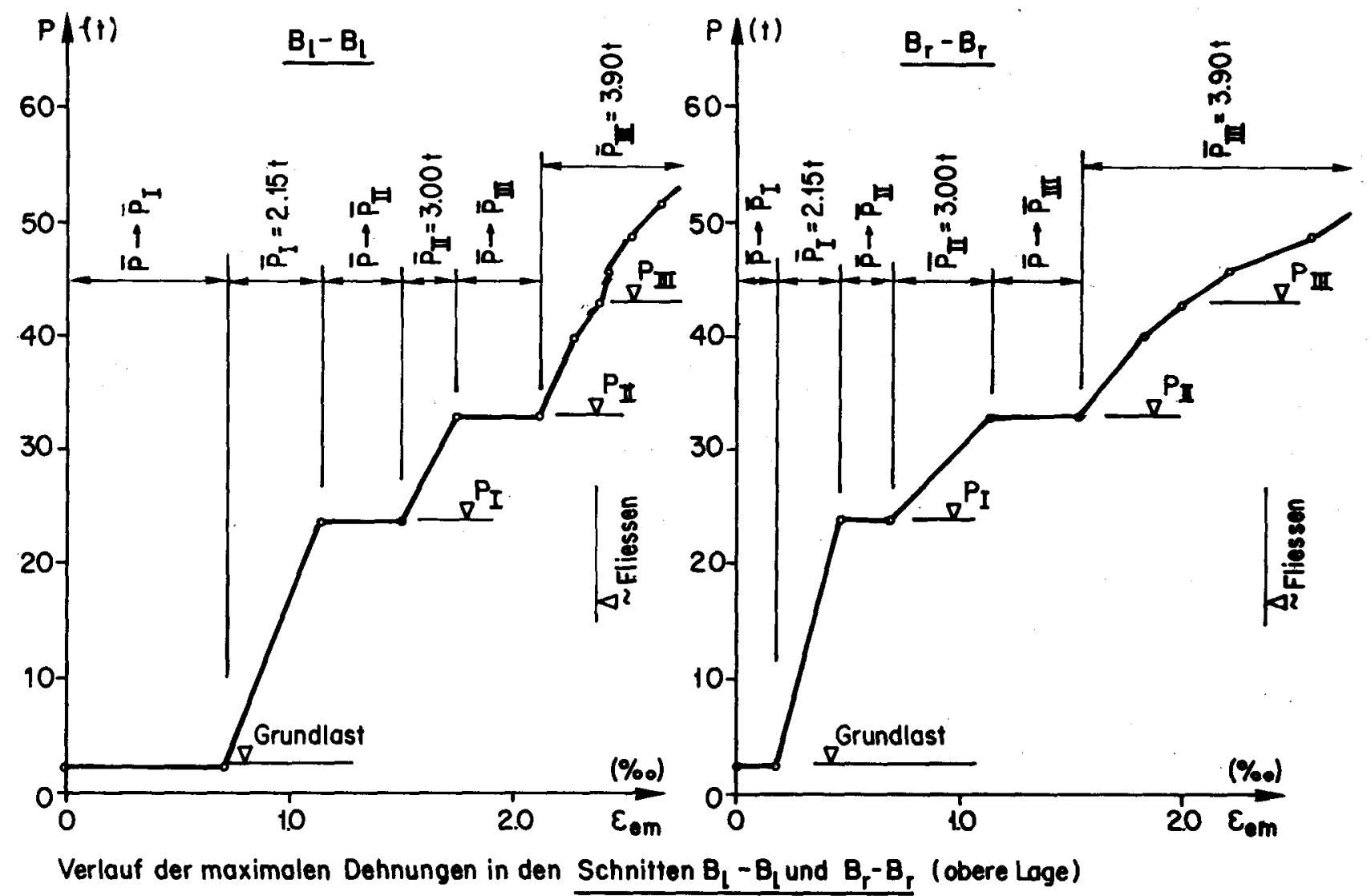

Bild 23-b : Dehnungen der Plattenquerarmierung beim Träger Z 5 


\begin{tabular}{|l|l|l|l|l|l|} 
II & II & II \\
\hline linke Seite: schlaff armiert & $\mathrm{Z} 5$ & rechte Seite: teilweise vorgesponnt \\
\hline
\end{tabular}
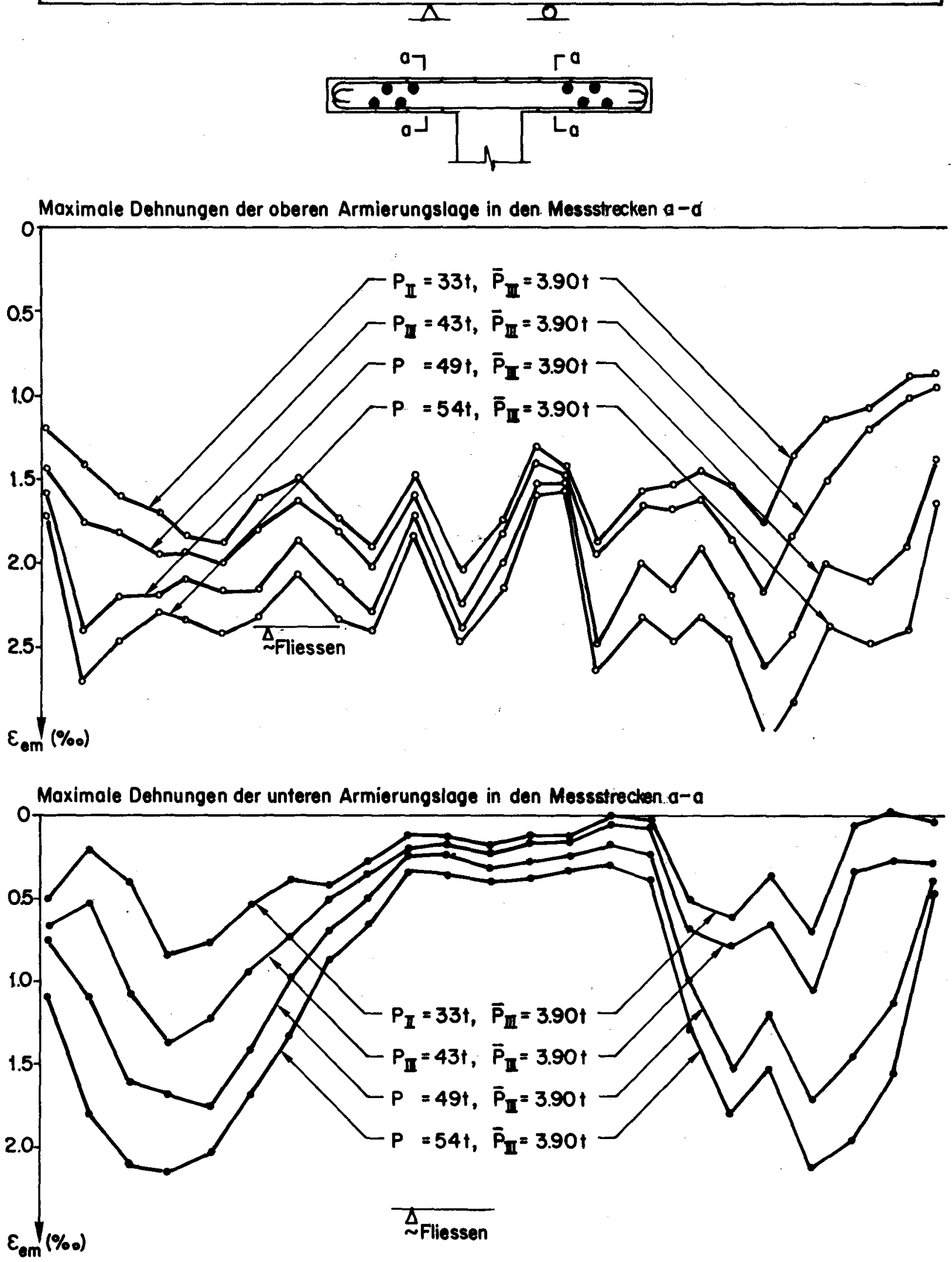

Bild 23-c: Dehnungen der Plattenquerarmierung beim Träger Z 5 

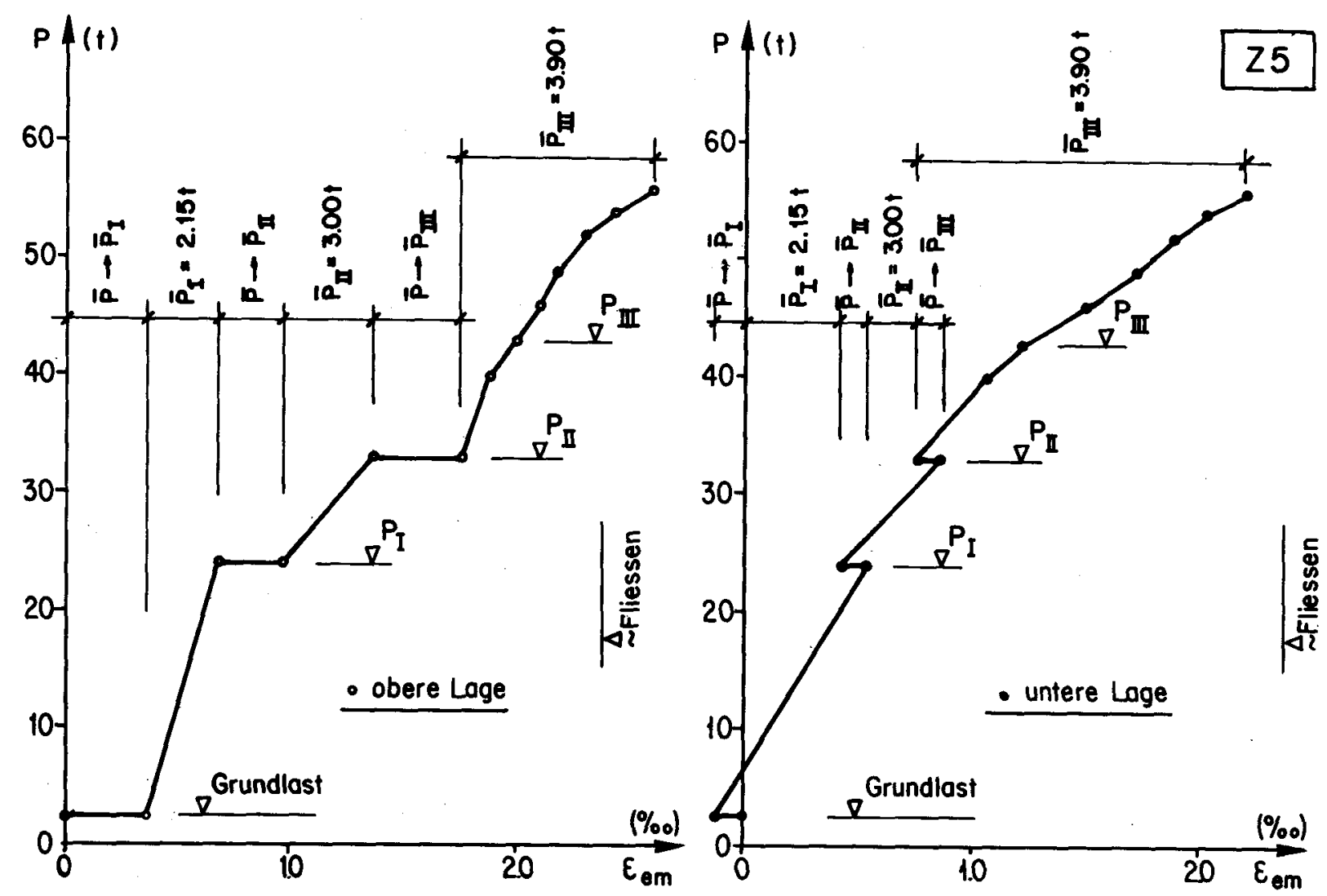

Verlauf der max. Dehnungen der Messstrecken $a-a$ im Schnitt $B_{1}-B_{l}$

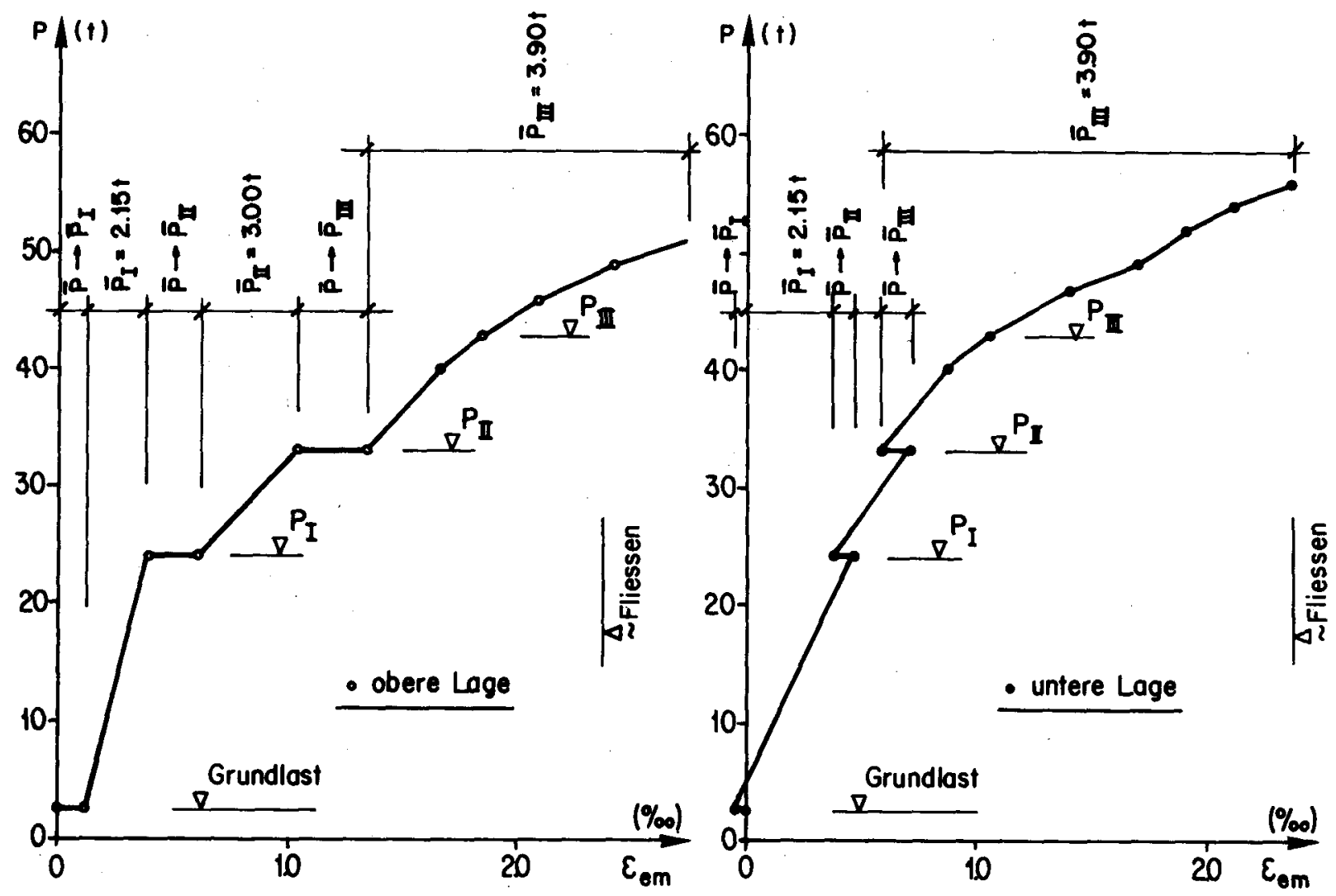

Verlouf der max. Dehnungen der Messstrecken $a-a$ im Schnitt $B_{r}-B_{r}$

Bild 23-d: Dehnungen der Plattenquerarmierung beim Träger Z5 


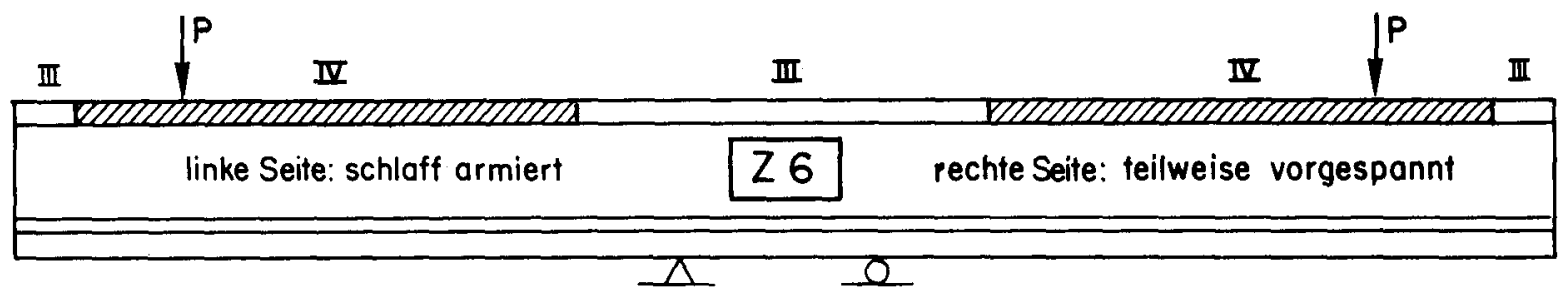
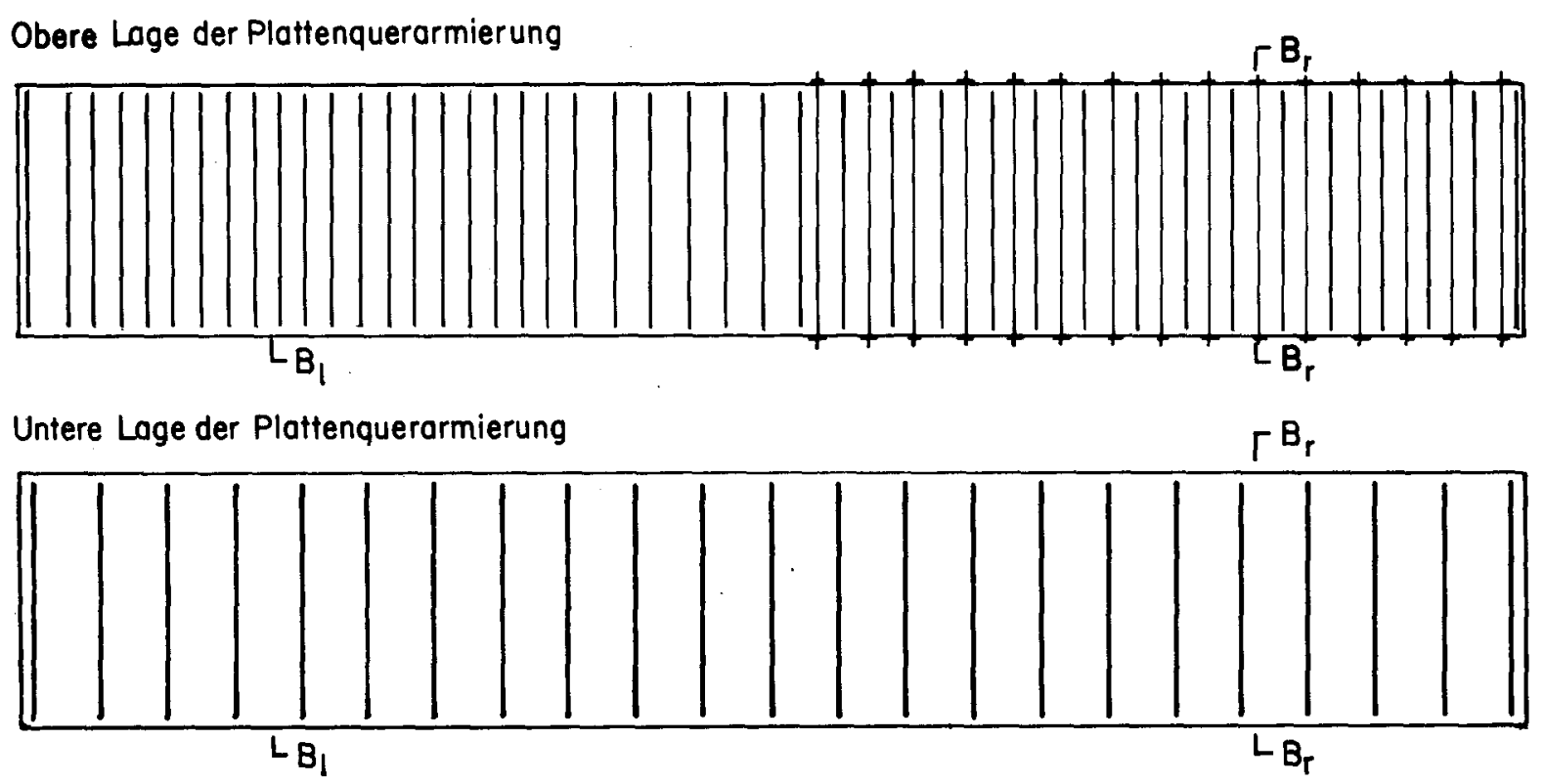

Maximale Dehnungen der oberen Armierungsiage

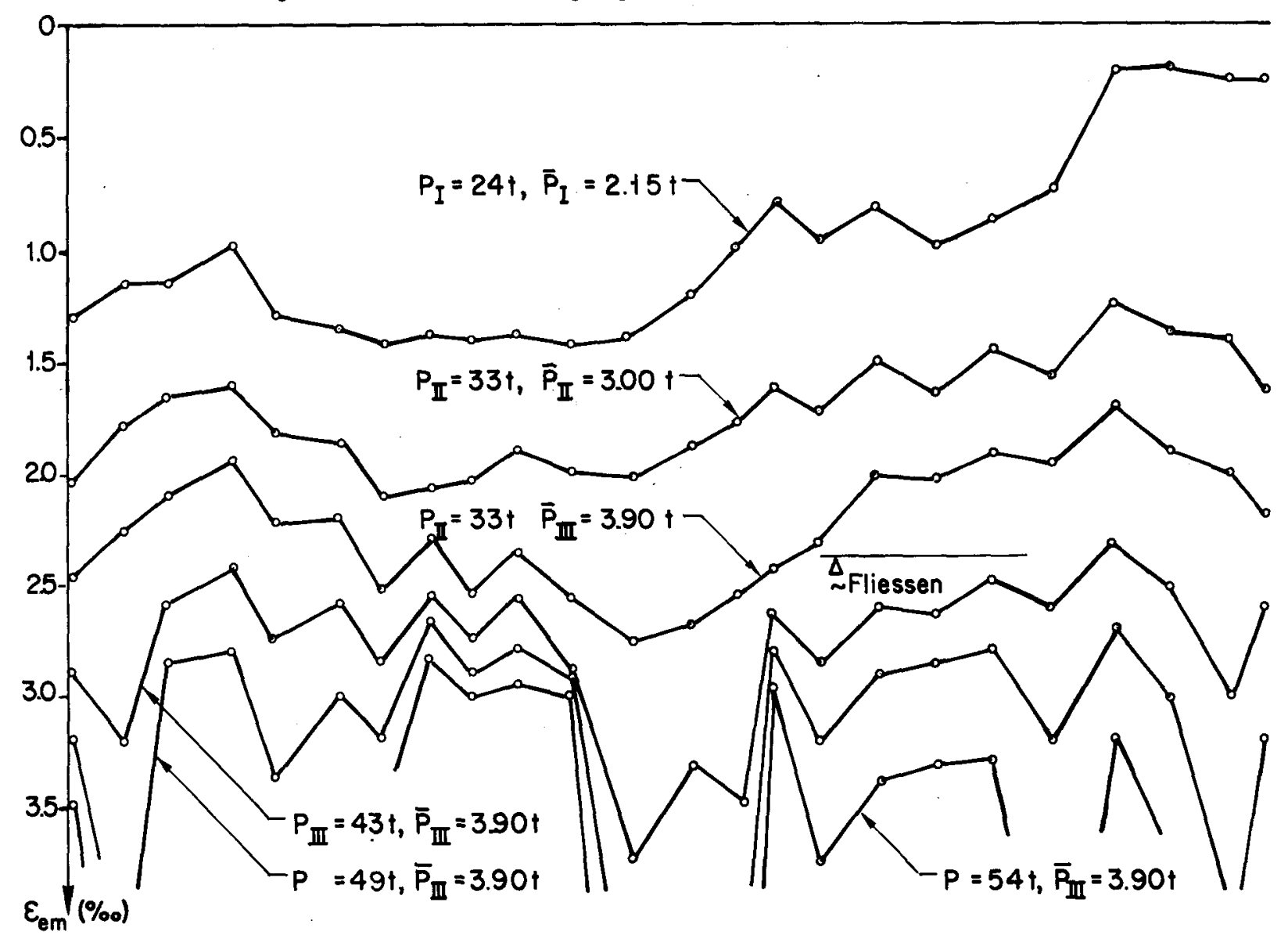

Bild 24-a: Dehnungen der Plattenquerarmierung beim Träger Z 6 

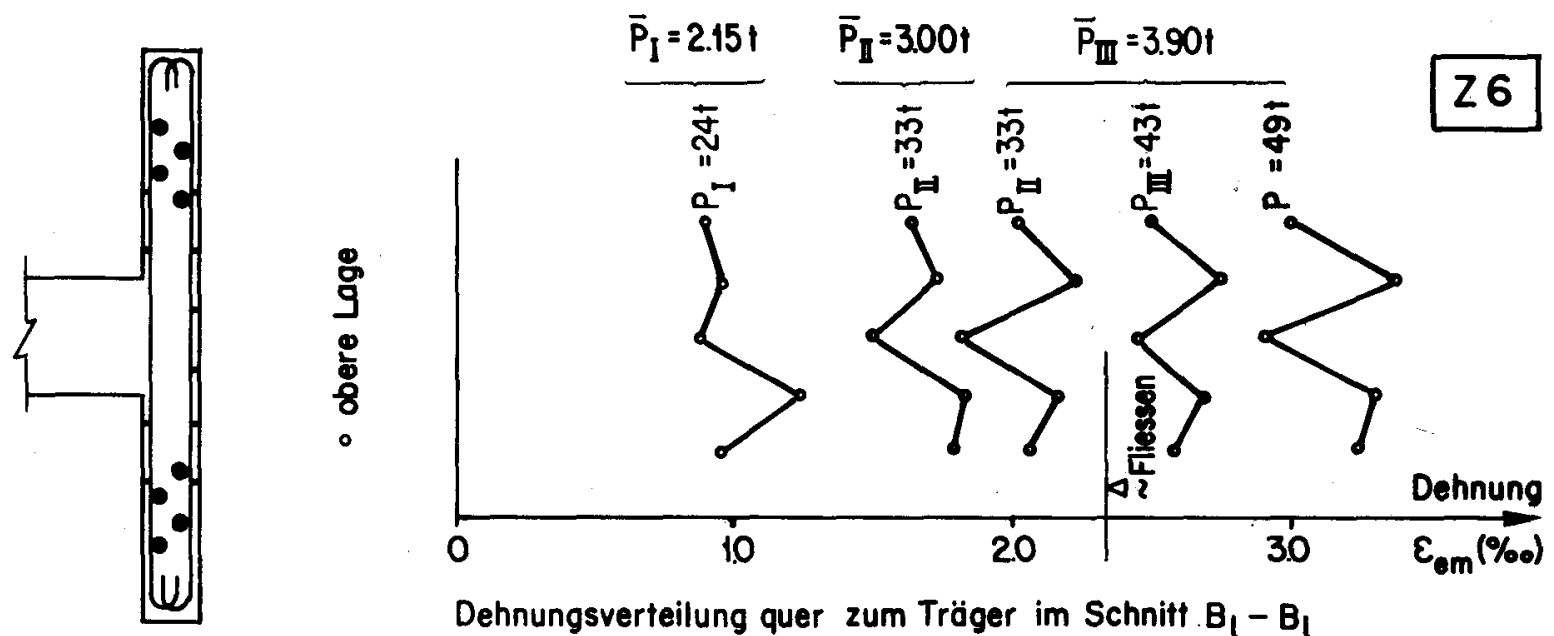

Dehnungsverteilung quer zum Träger im Schnitf $B_{1}-B_{1}$
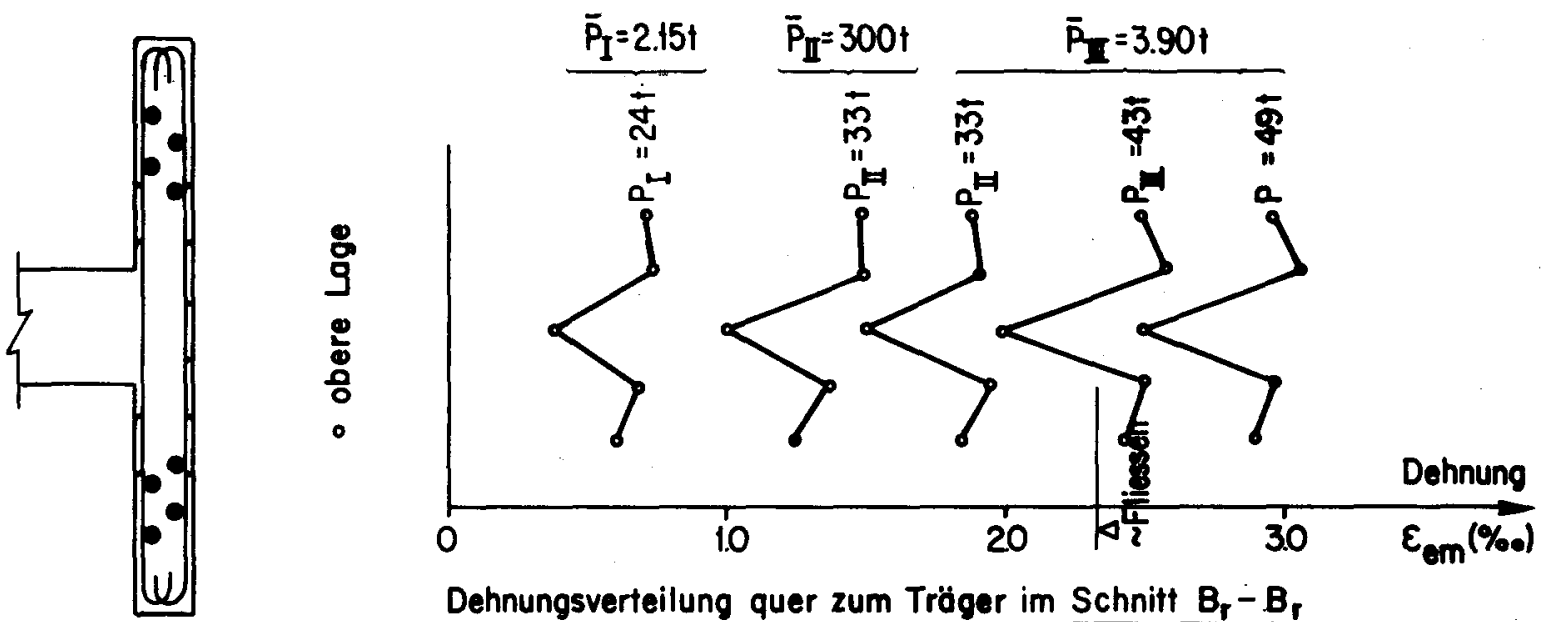

Dehnungsverteilung quer zum Träger im Schnitt $B_{r}-B_{r}$

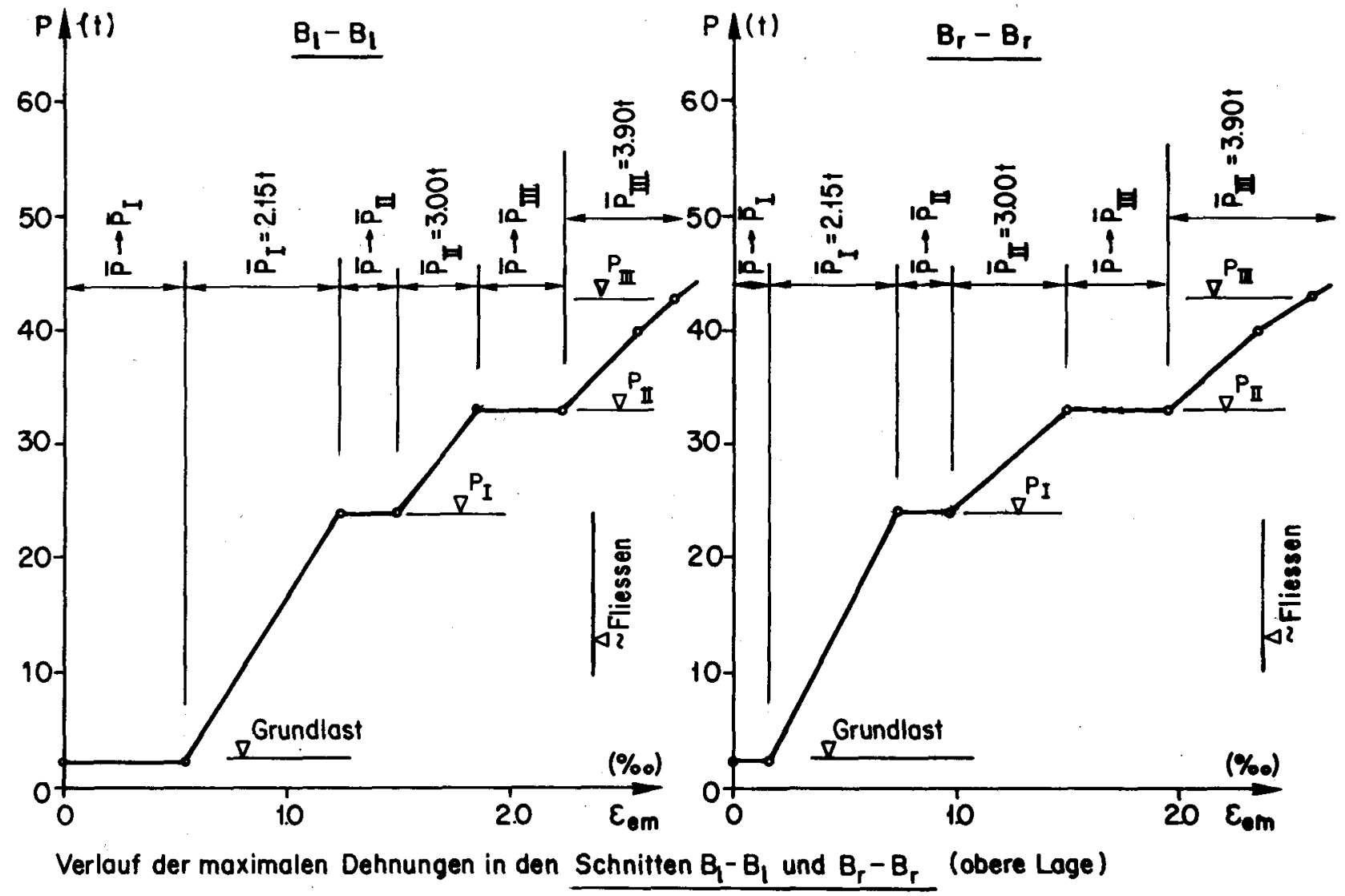

Bild 24-b : Dehnungen der Plattenquerarmierung beim Träger 26 

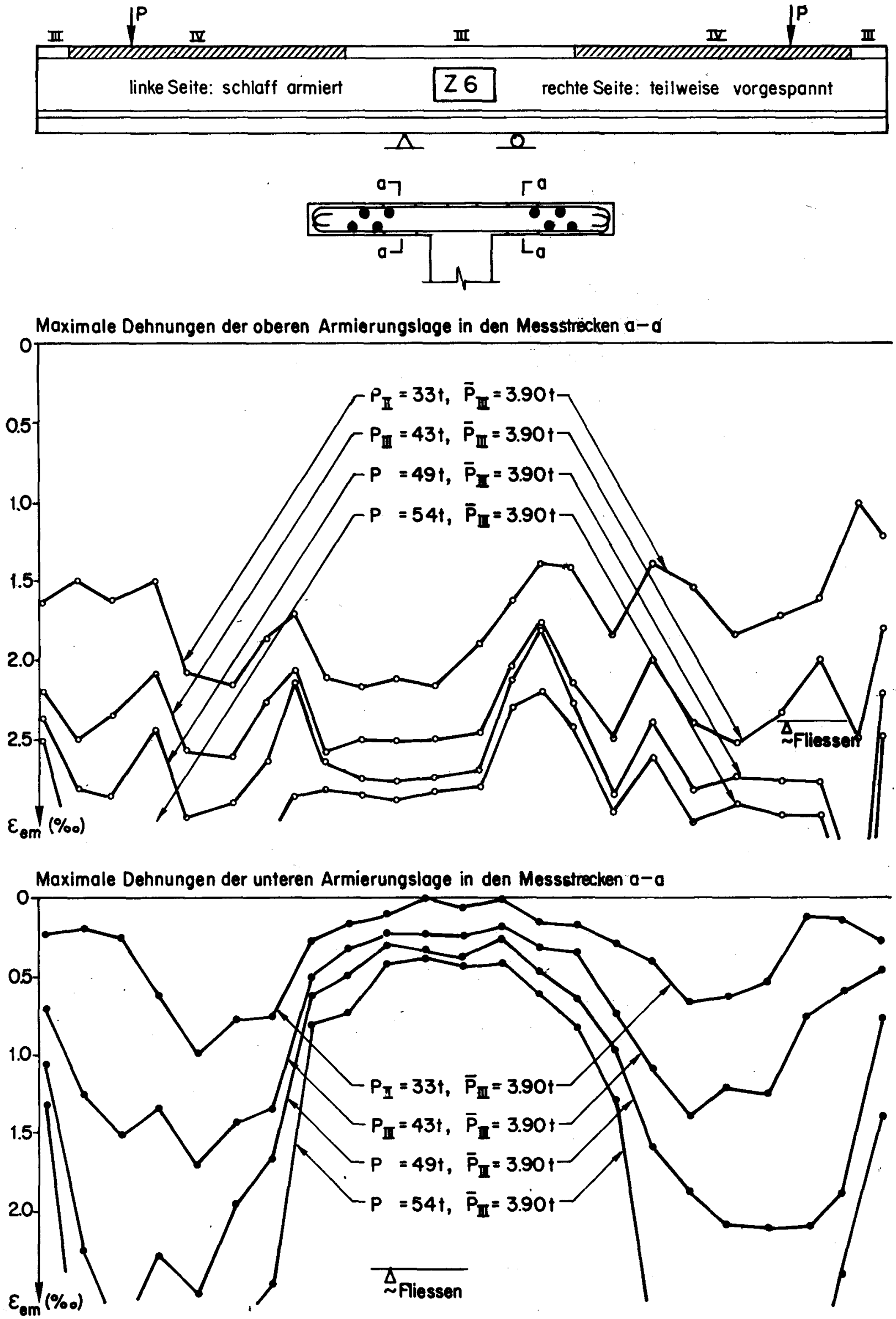

Bild 24-c: Dehnungen der Plattenquerarmierung beim Träger Z 6 


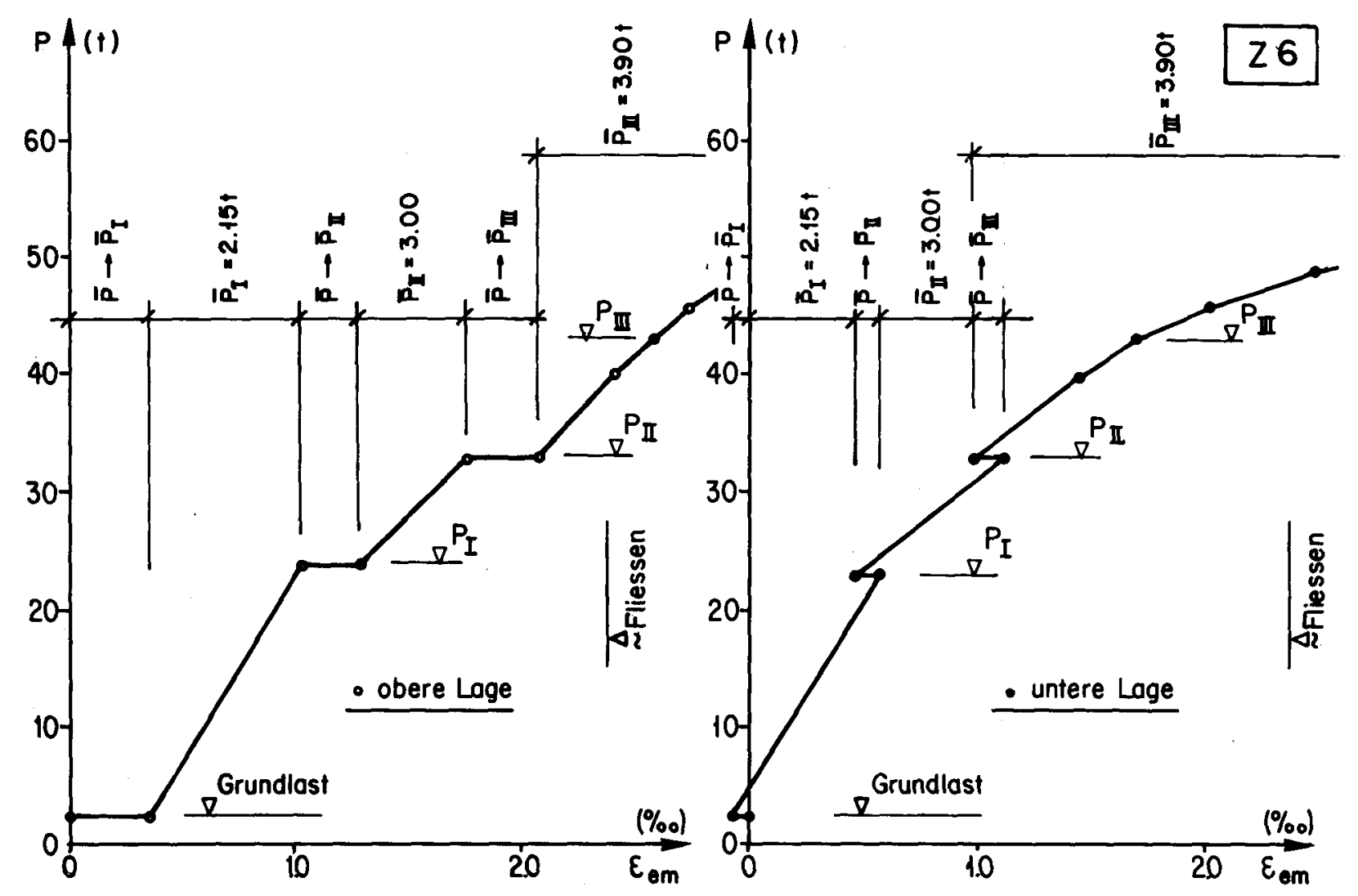

Verlauf der max. Dehnungen der Messstrecken $a-a$ im Schnitt $B_{1}-B_{l}$

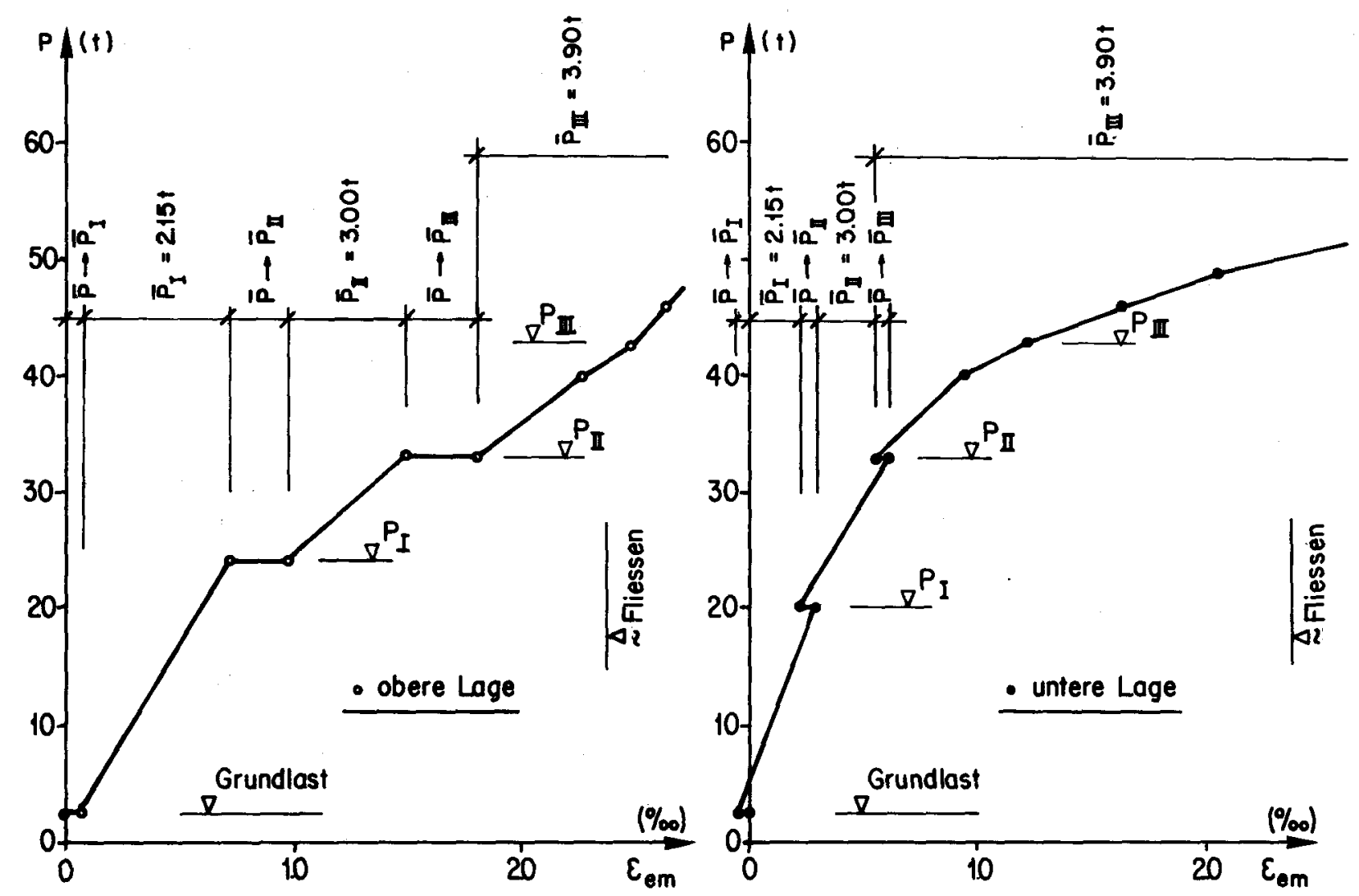

Verlauf der max. Dehnungen der Messstrecken $a-a$ im Schnitt $B_{r}-B_{r}$

Bild 24-d; Dehnungen der Plattenquerarmierung beim Träger 26 


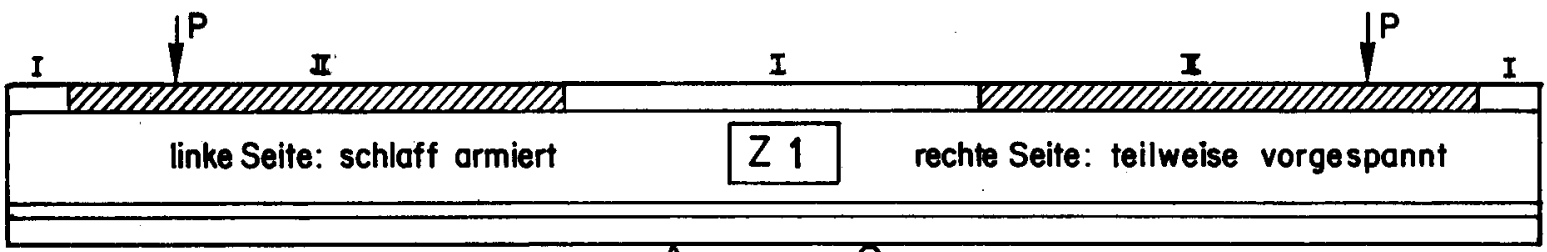

스

Kräfteverlauf in der oberen Plattenquerarmierung

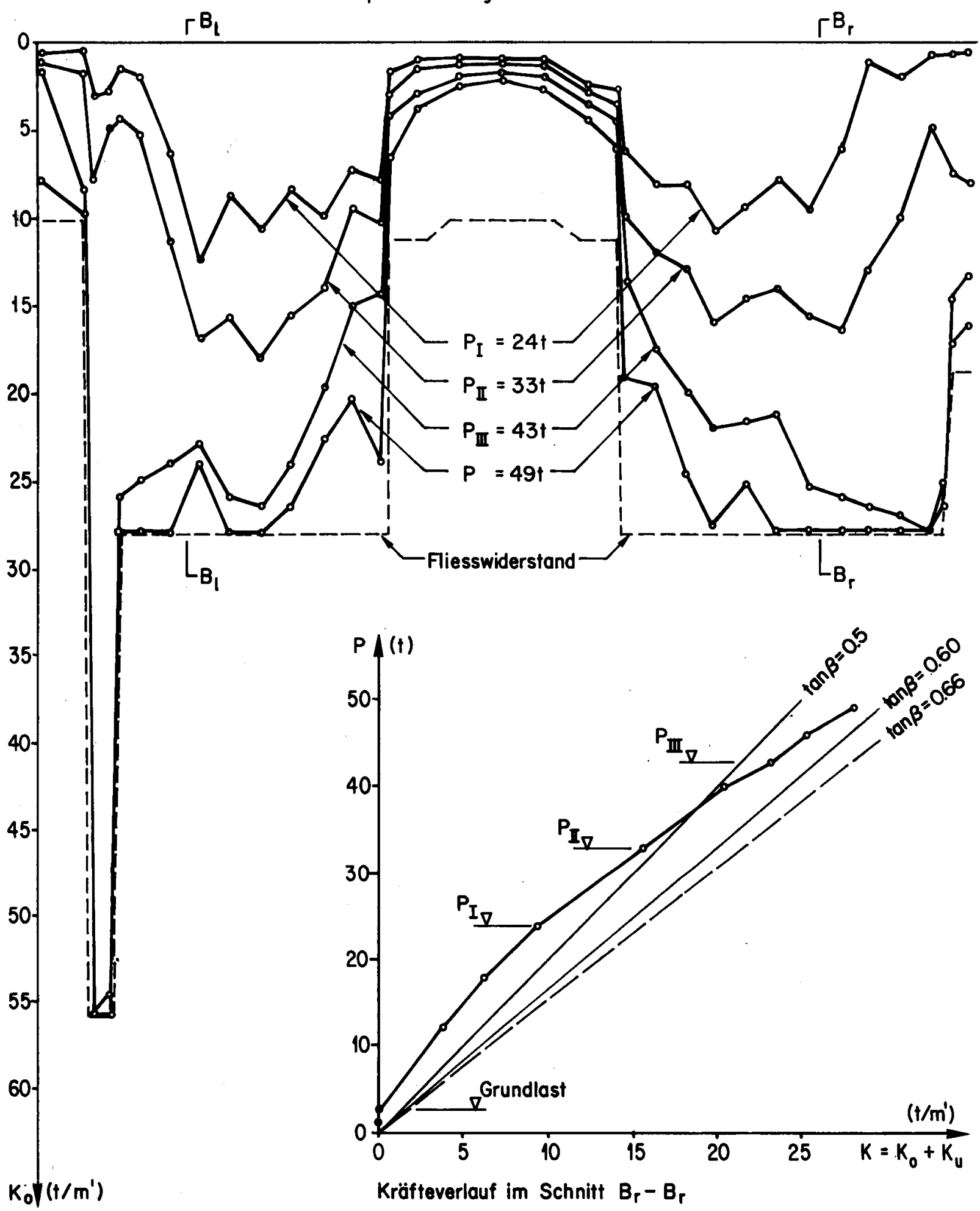

Bild 25: Kräfteverlauf in der Plattenquerarmierung beim Träger Z1 


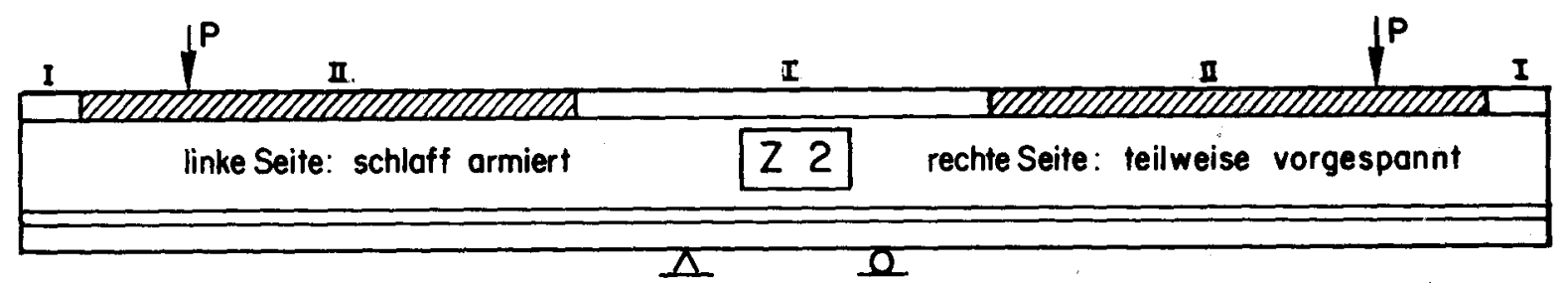

Kräftequerlauf in der oberen Plattenquerarmierung
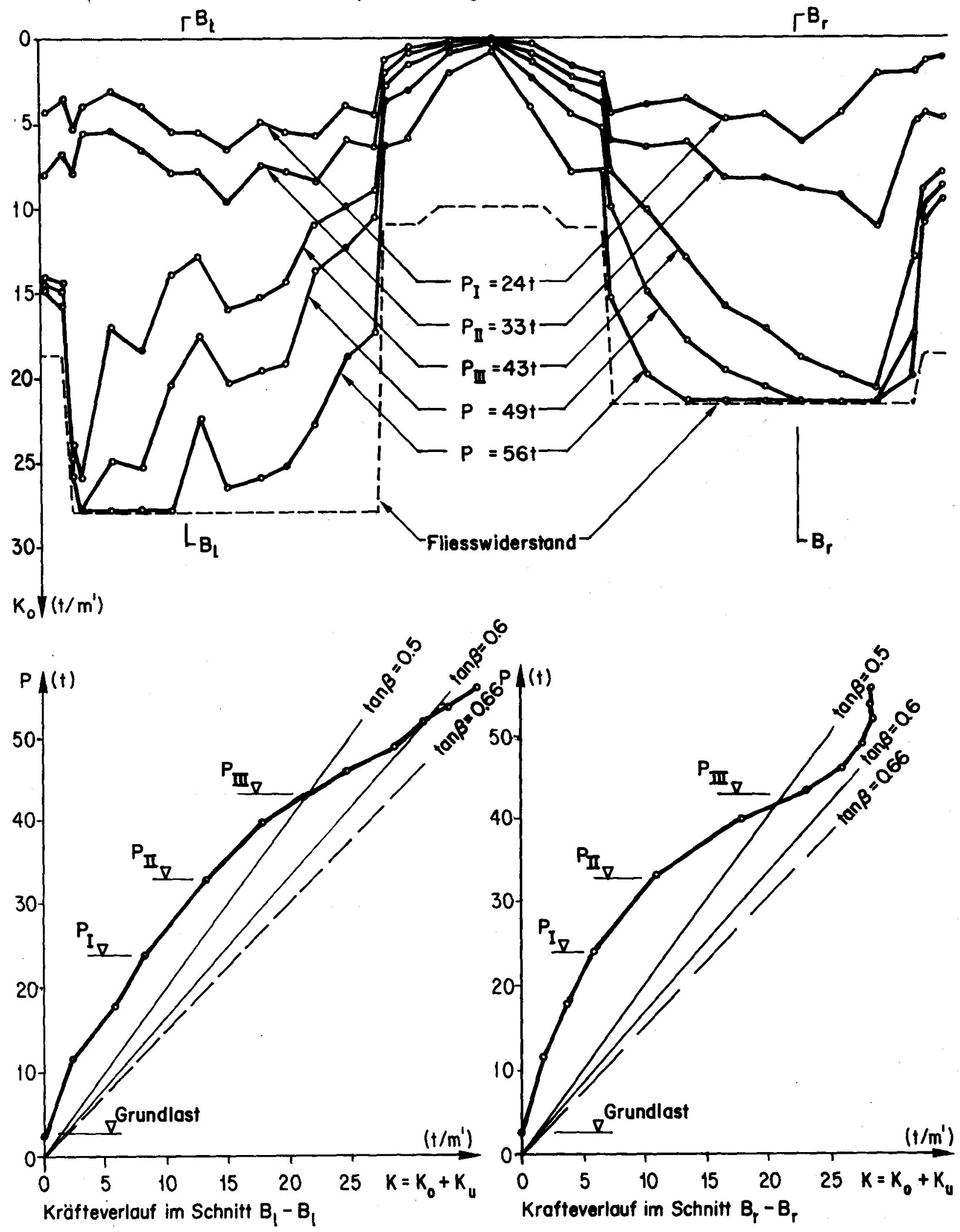

Bild 26: Kräfteverlauf in der Plattenquerarmierung beim Träger $\mathbf{Z} 2$ 


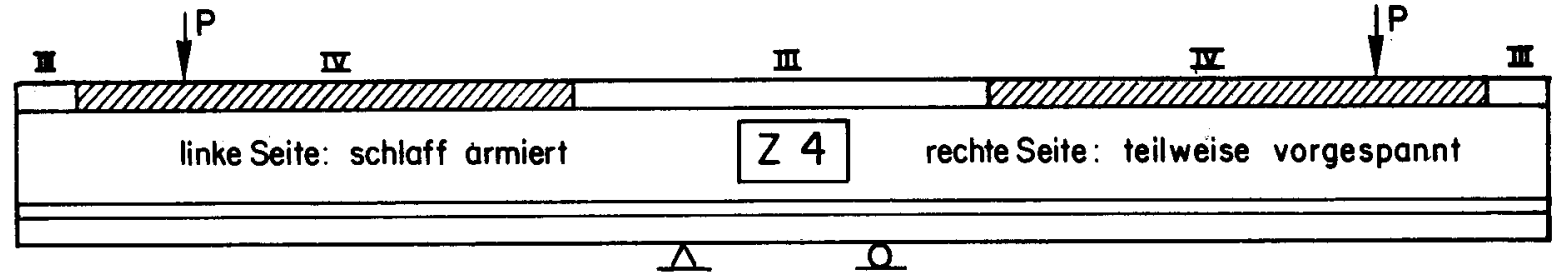

Kröfteverlauf in der oberen Plattenquerarmierung unter Längsschub allein
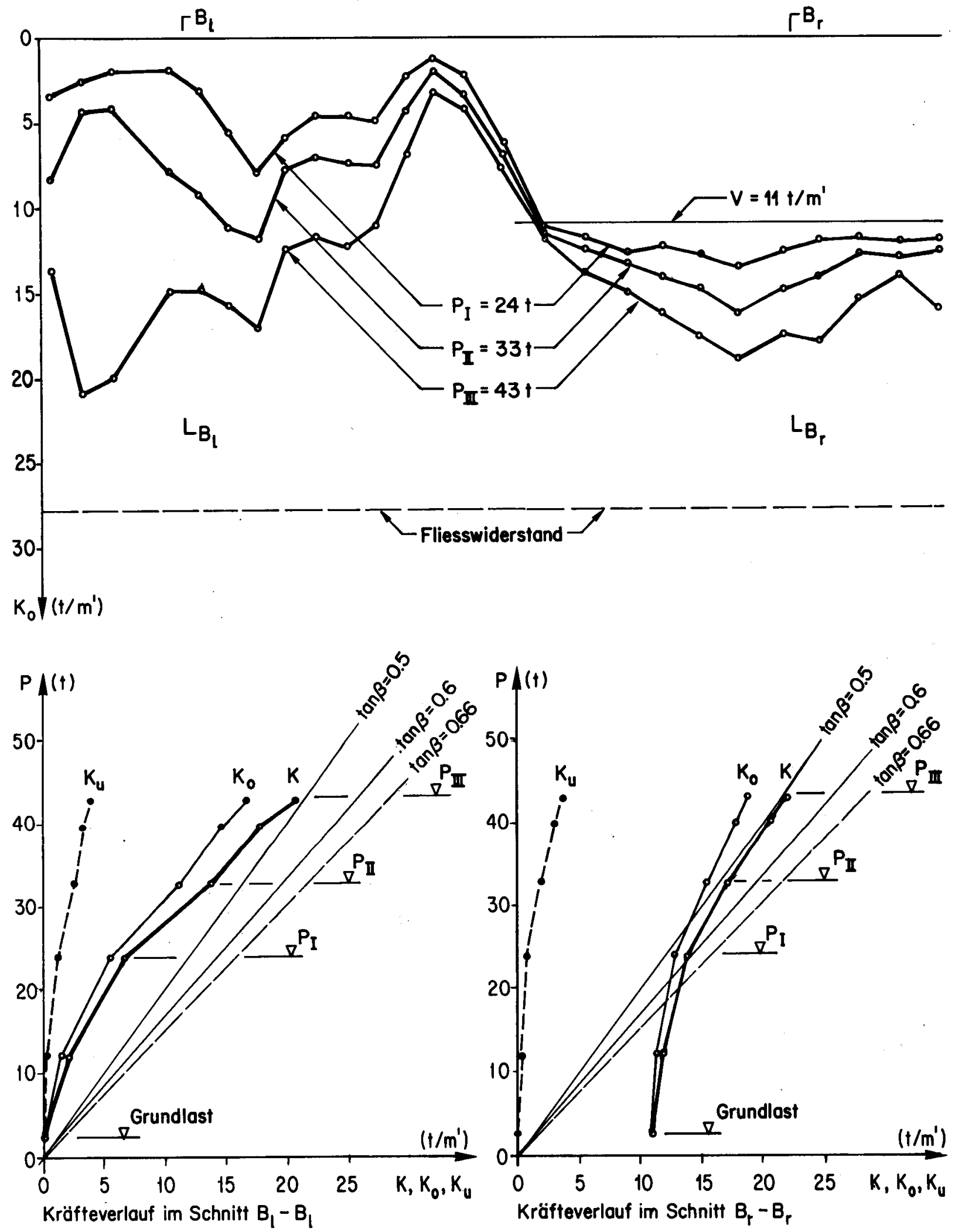

Bild 27-a: Kräffeverlauf unter längsschub allein beim Träger Z 4 


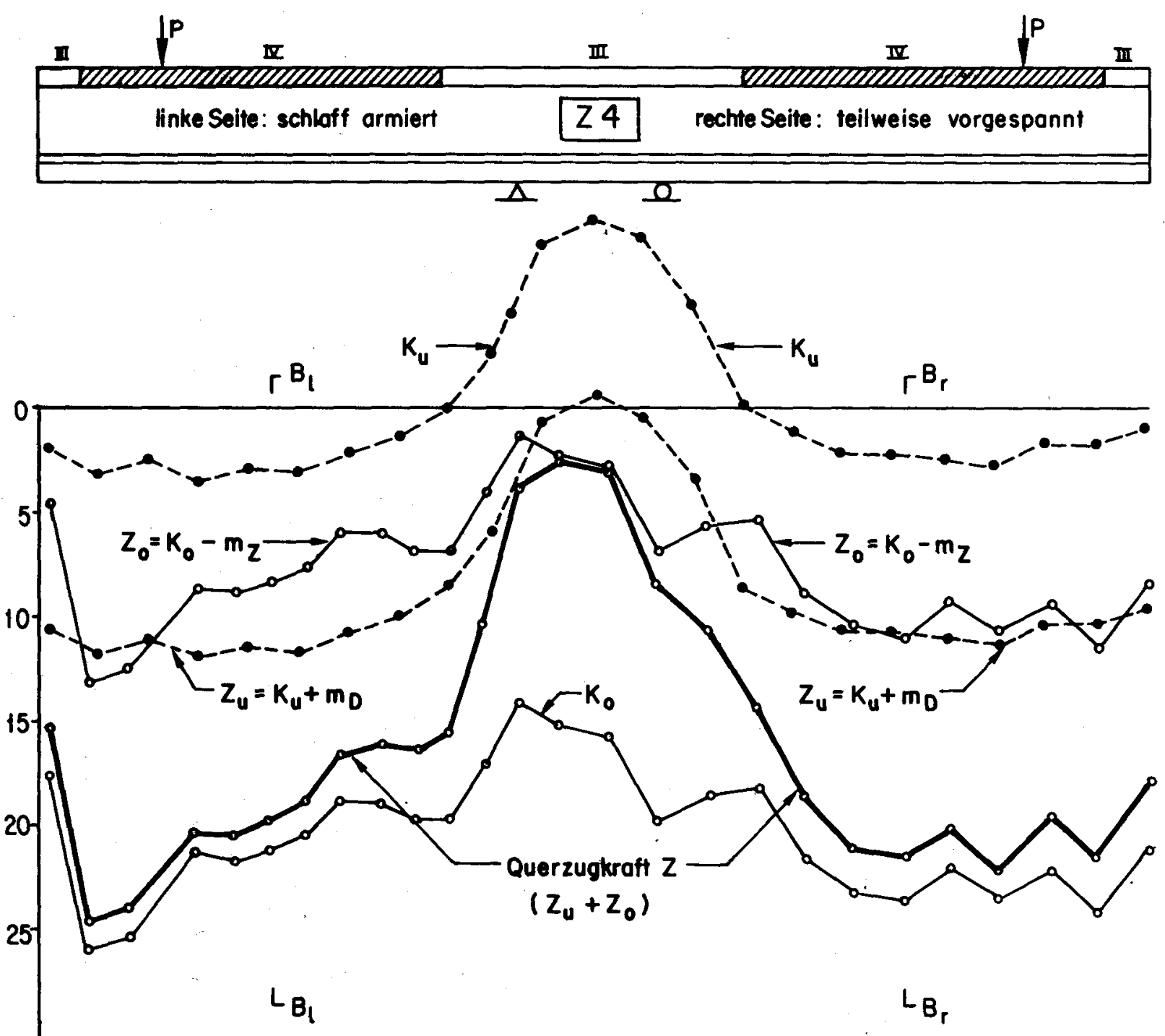

$K Z /\left(t / m^{\prime}\right)$ Kräfteverlauf in den Messstrecken a-a bei $P_{\text {II }}=43 t, \bar{P}_{I}=3.30 t$

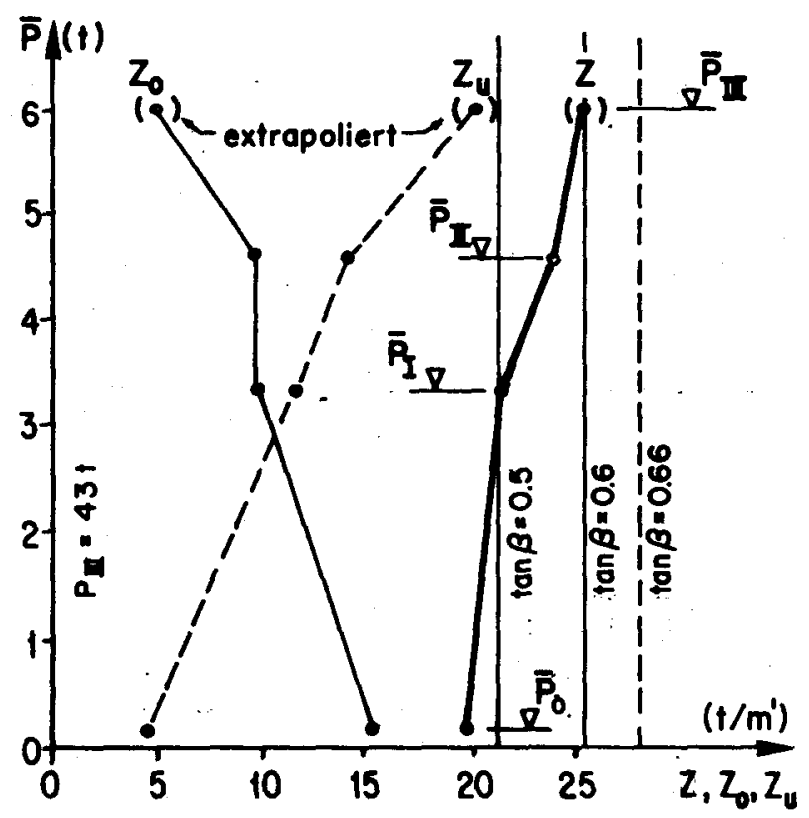

Kraffteverlouf im Schnitt $B_{l}-B_{l}$

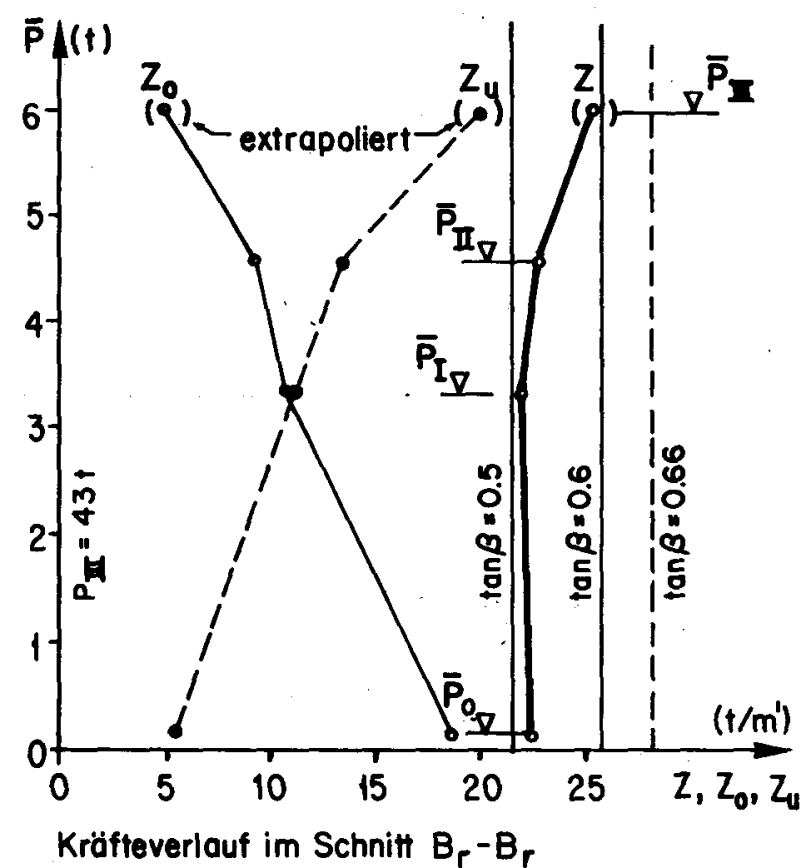

Bild 27-b: Kräfteverlauf in den Messstrecken a- $a$ beim Träger Z 4 


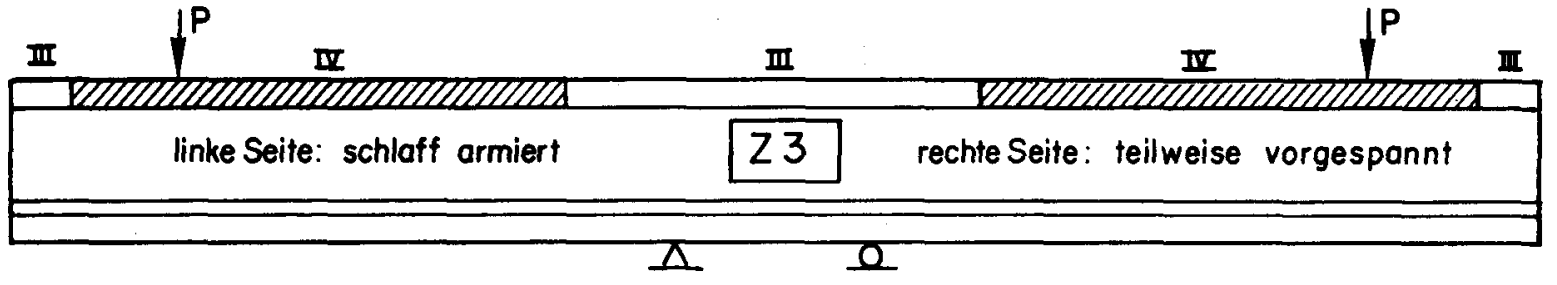

Kräfteverlouf in der oberen Plattenquerarmierung
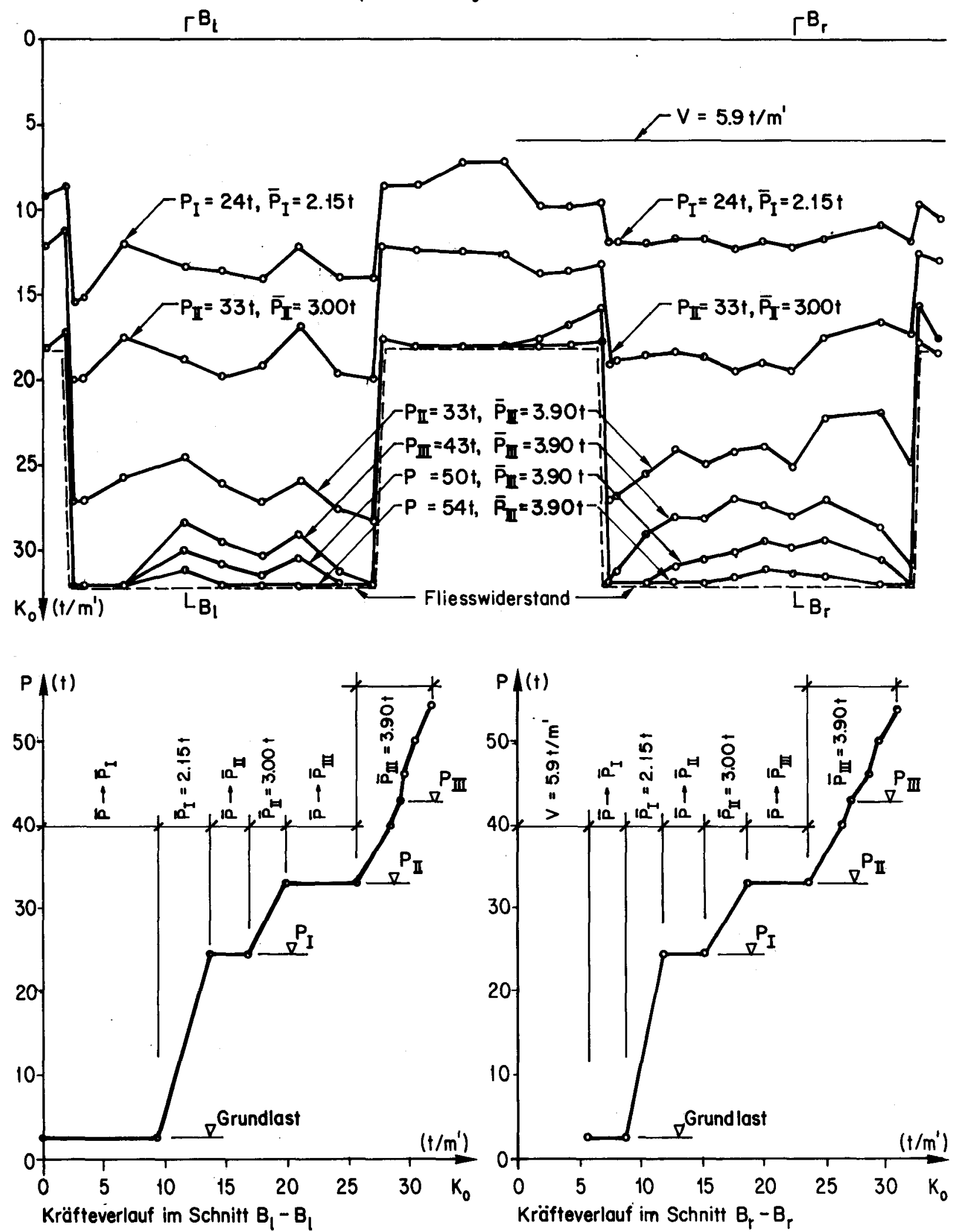

Bild 28-a: Kräfteverlauf in der oberen Plattenquerarmierung beim Träger $Z 3$ 

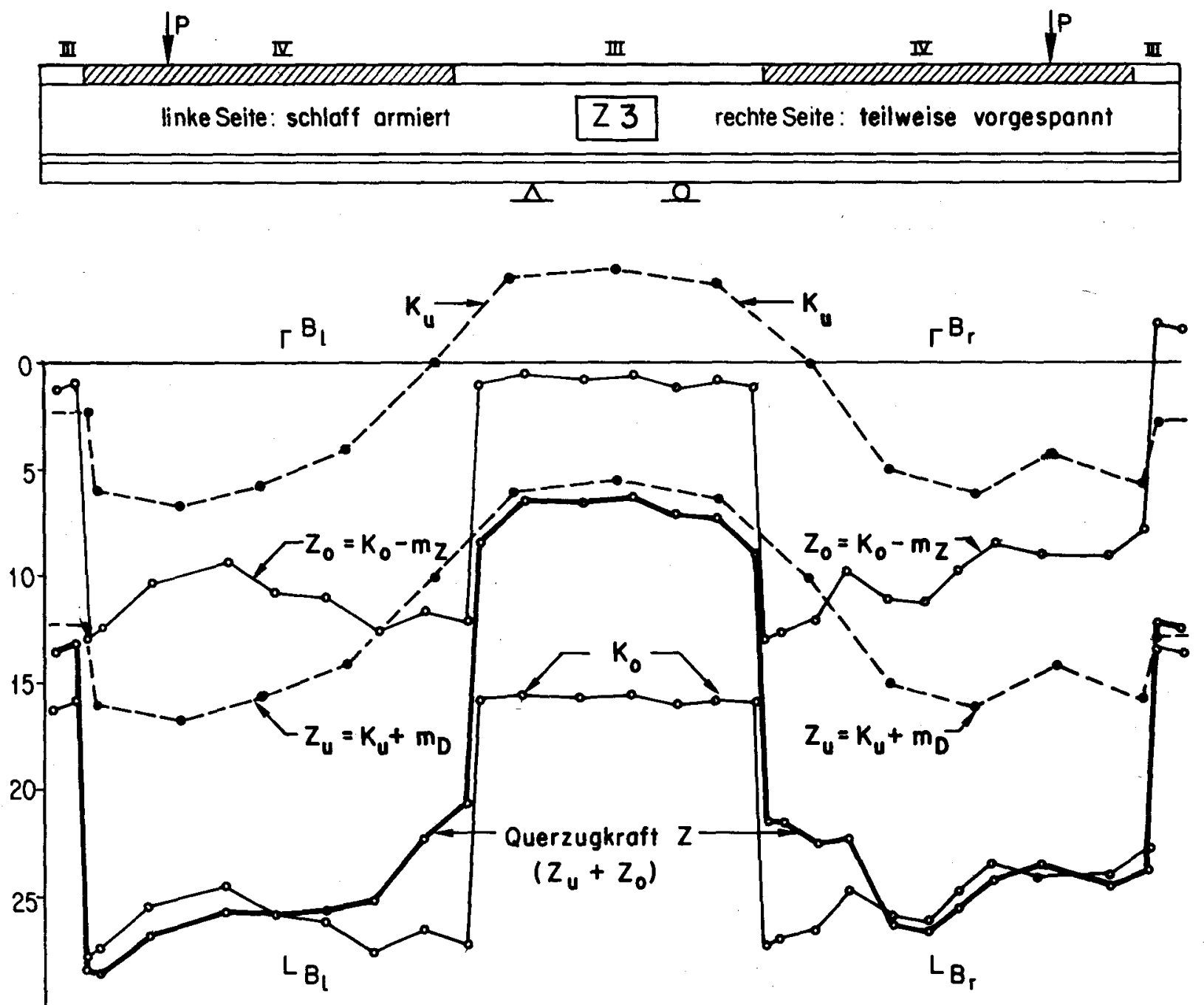

$K, Z \gamma\left(t / m^{\prime}\right) \quad$ Kräfteverlauf in den Messstrecken $a-a$ bei $P_{I}=43 t, \bar{P}_{I}=3.90 t$
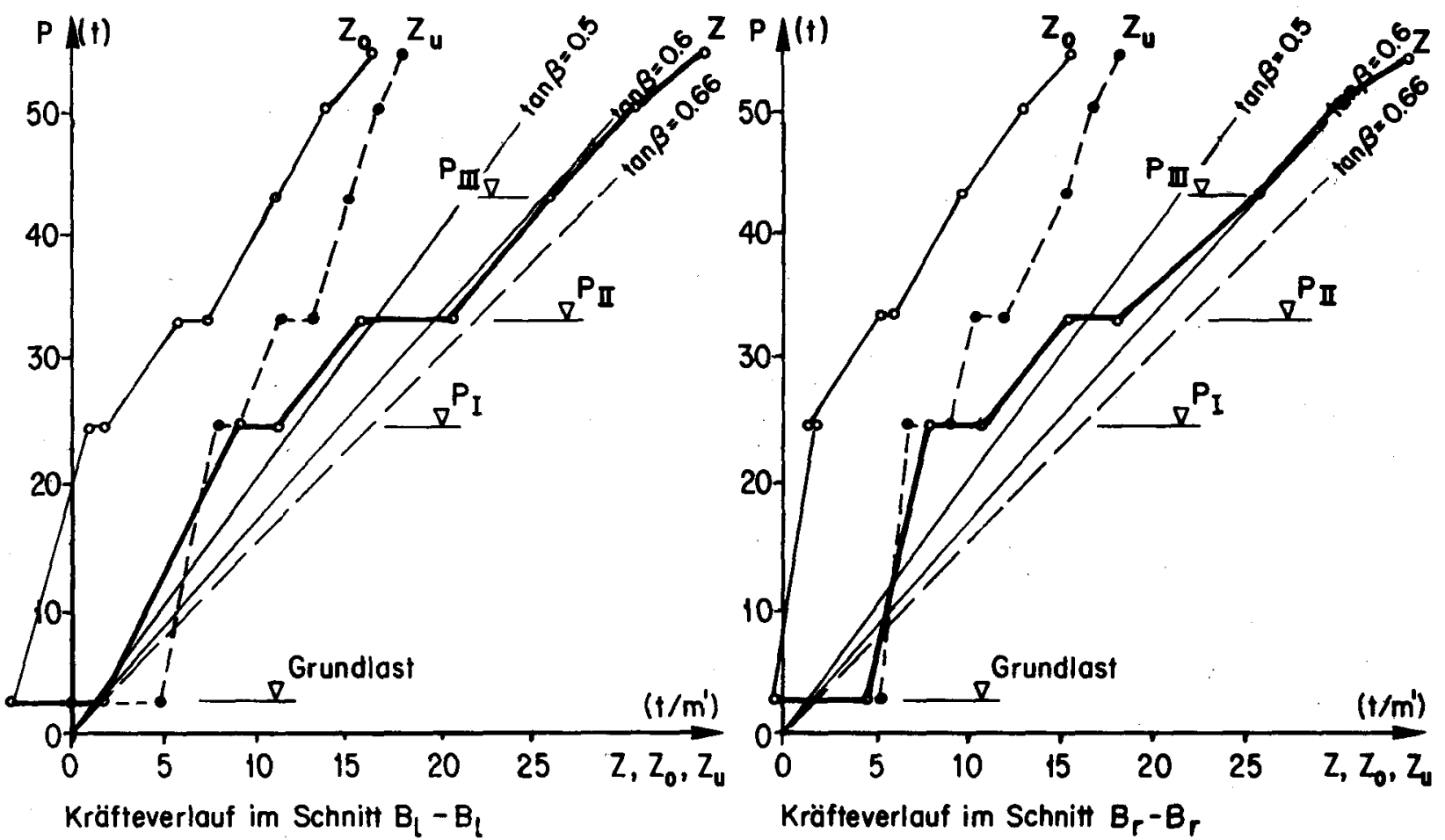

Bild 28-b: Kräfteverlauf in den Messstrecken a- $a$ beim Träger Z 3 


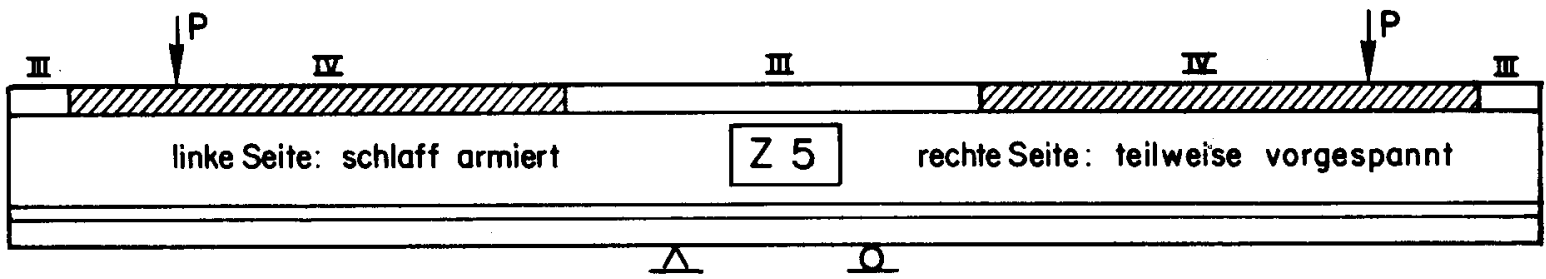

Kräfteverlauf in der oberen Plattenquerarmierung
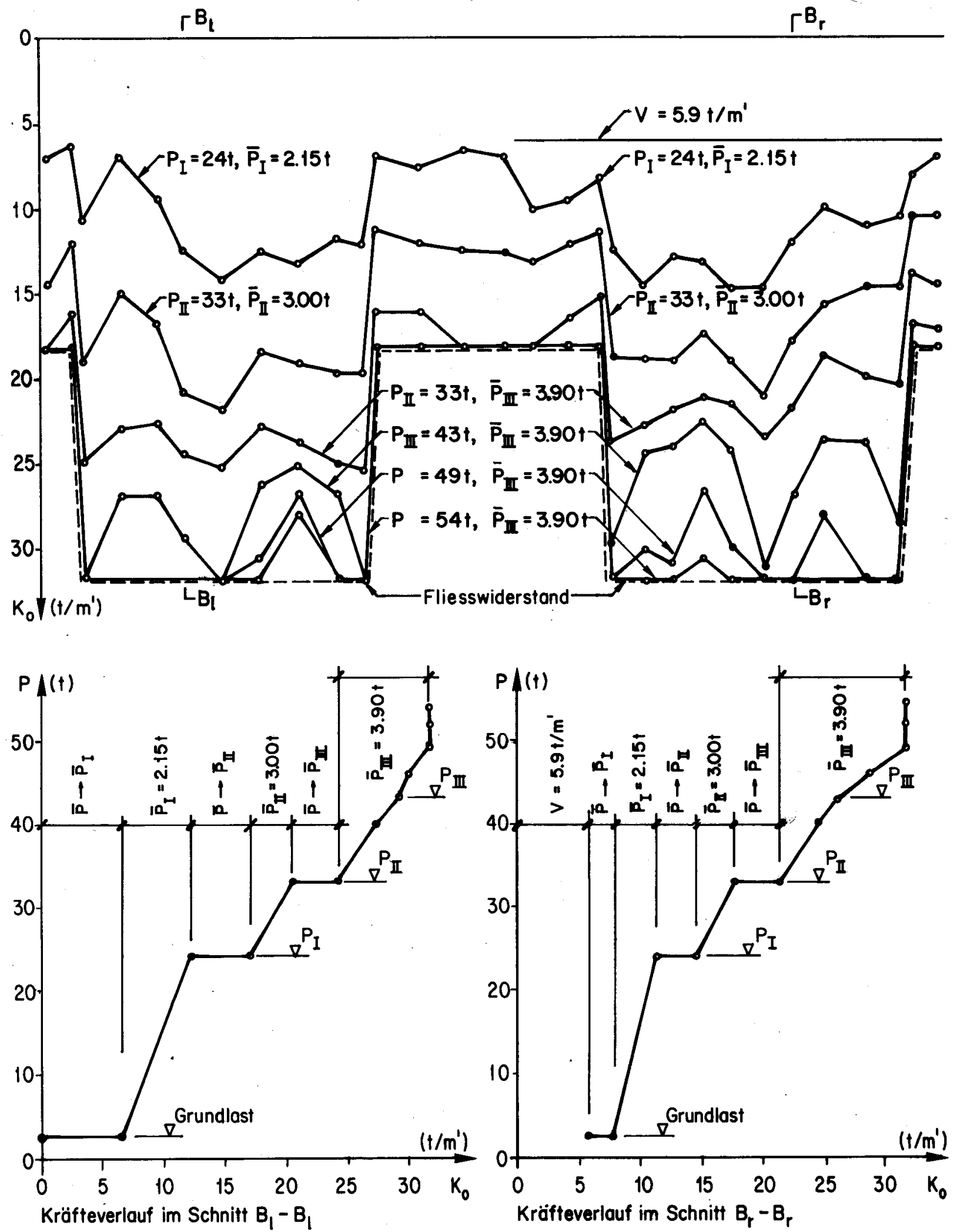

Bild 29-a: Kräfteverlauf in der oberen Plattenquerarmierung beim Träger Z 5 

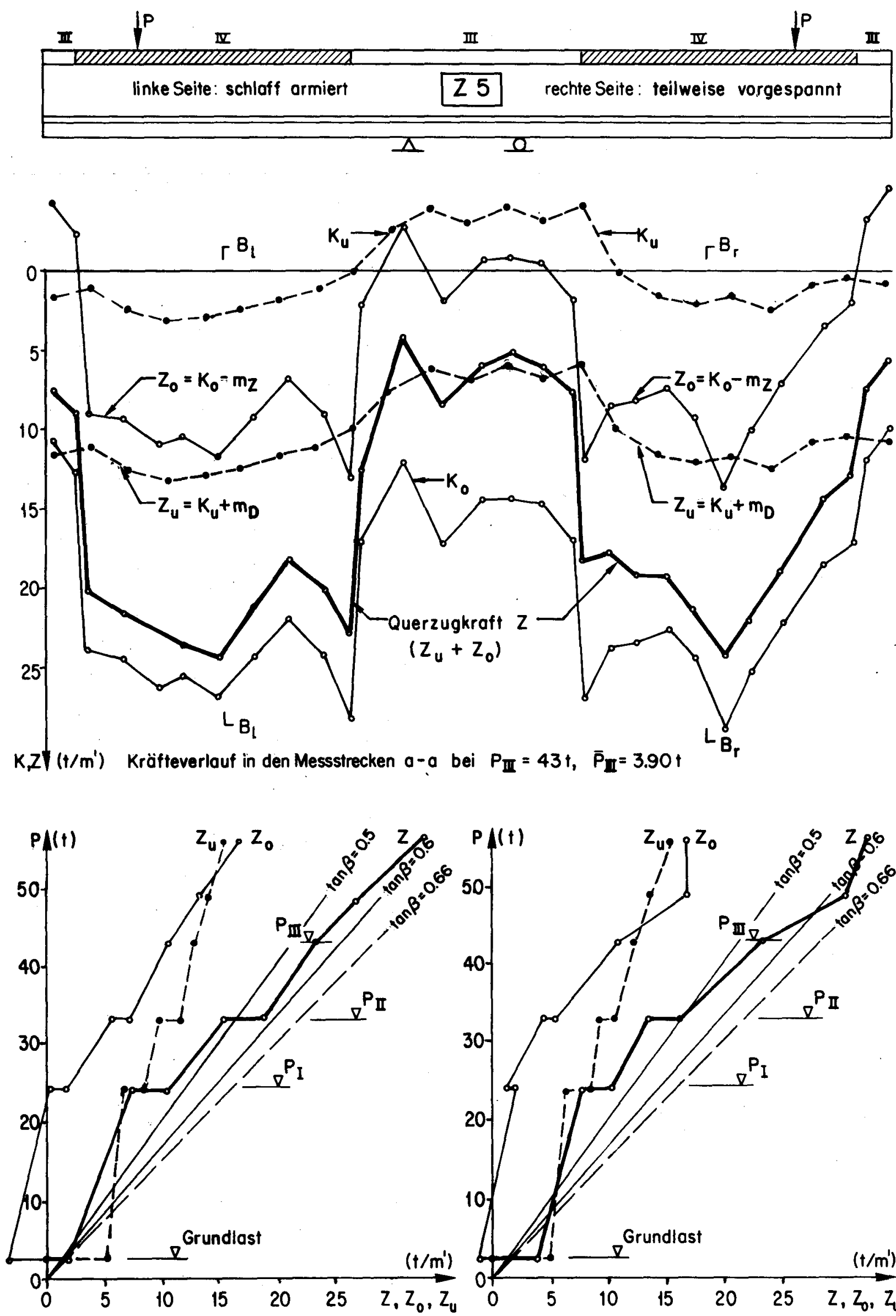

Kräfteverlauf im Schnitt $B_{l}-B_{l}$

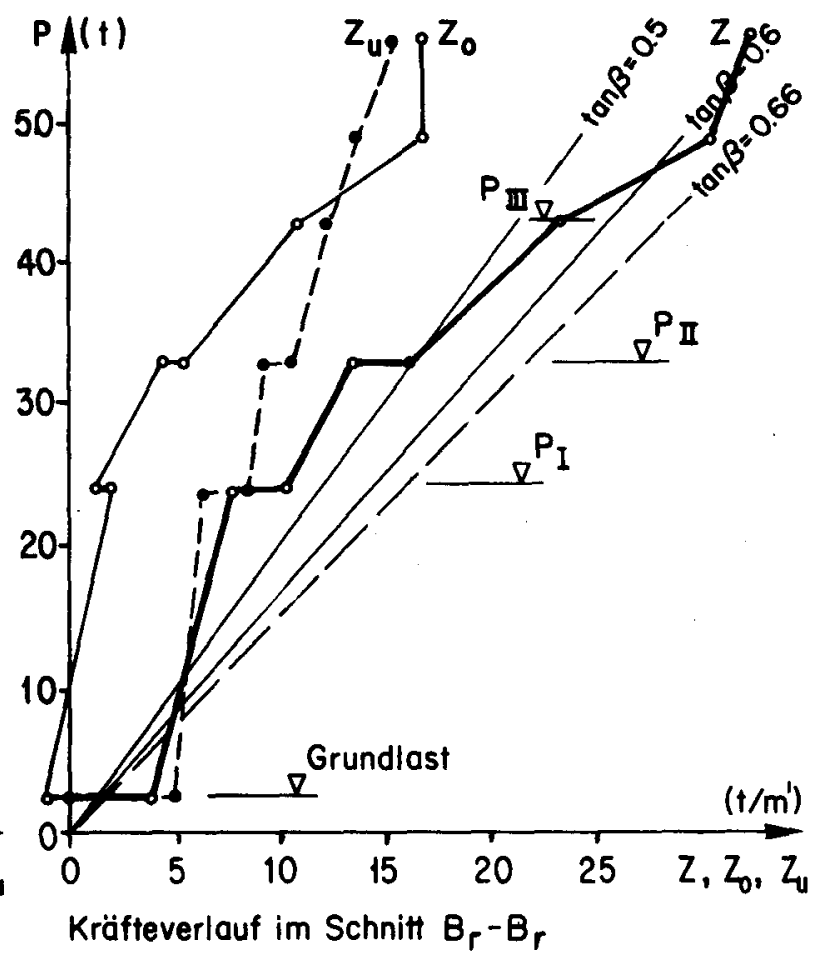

Bild 29-b: Kräfteverlauf in den Messstrecken a- $a$ beim Träger Z 5 


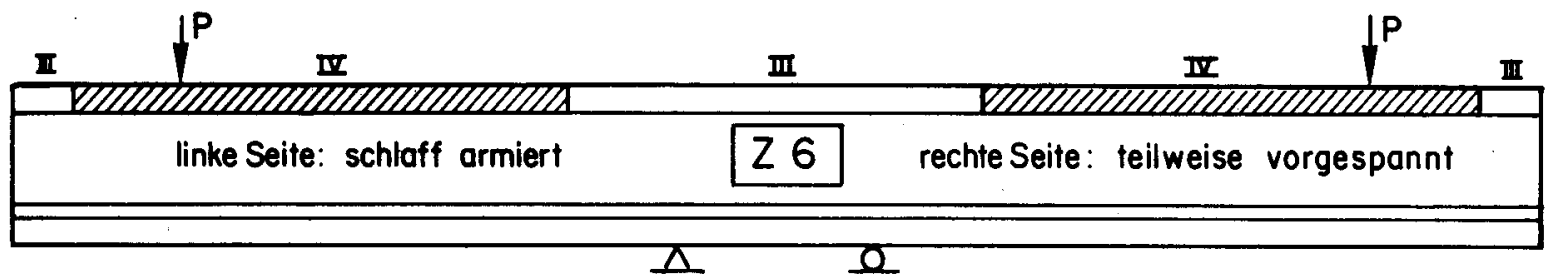

Kröfteverlauf in der oberen Plattenquerarmierung
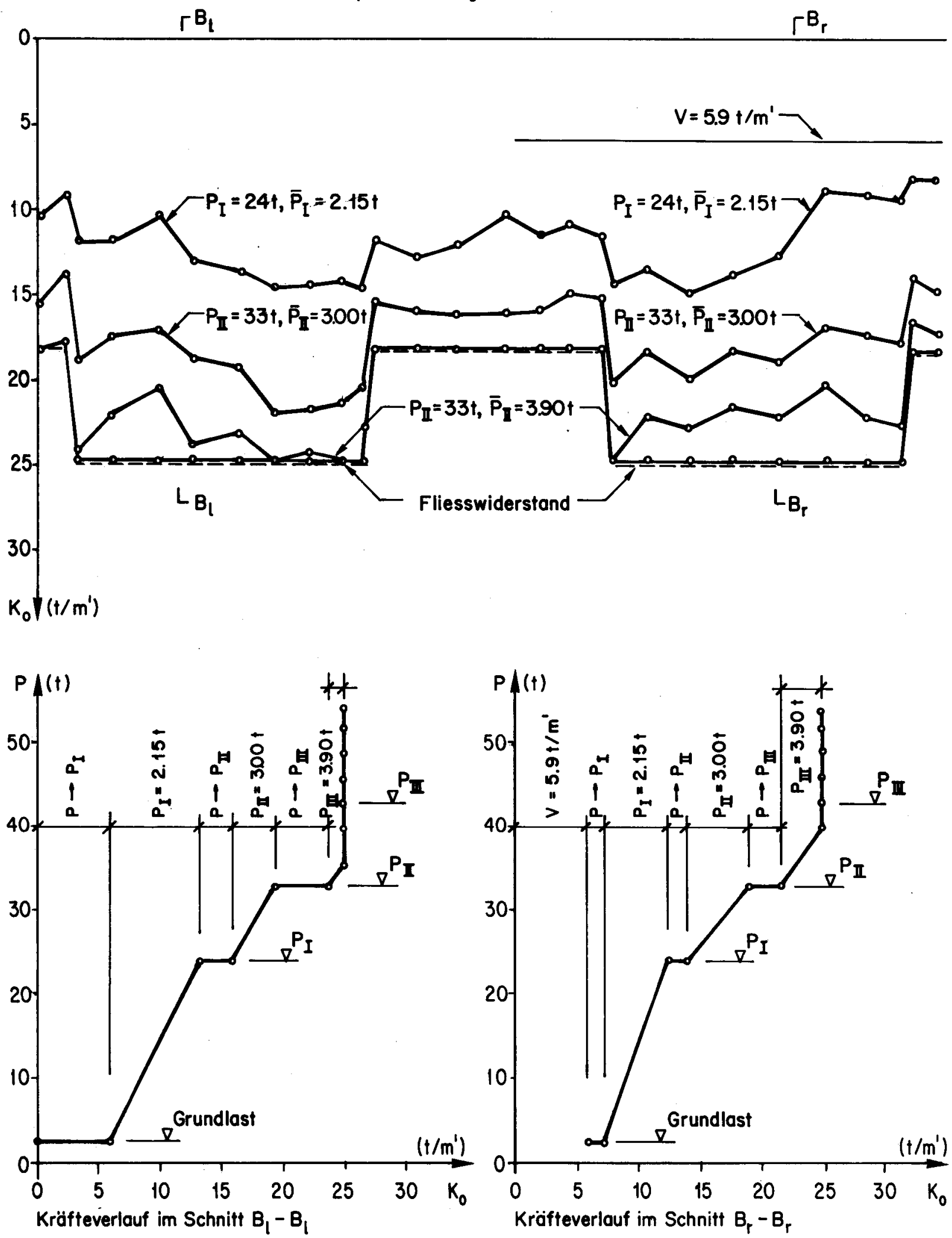

Bild 30-a: Kräfteverlauf in der oberen Plattenquerarmierung beim Träger $Z 6$ 

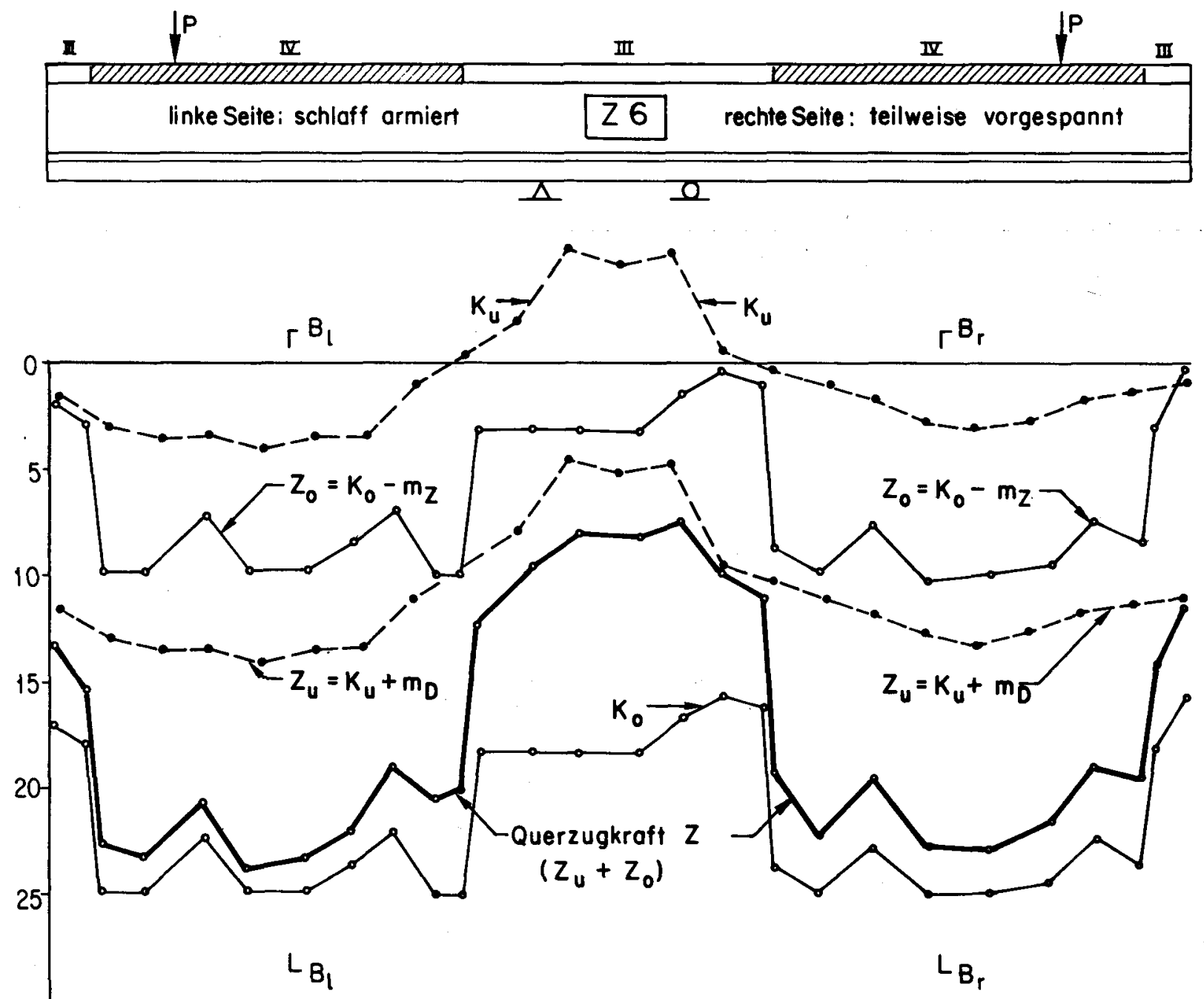

$K, Z)\left(t / m^{\prime}\right) \quad$ Kräfteverlouf in den Messstrecken a-a bei $P_{\text {III }}=43 t, \bar{P}_{\text {II }}=3.90 \dagger$
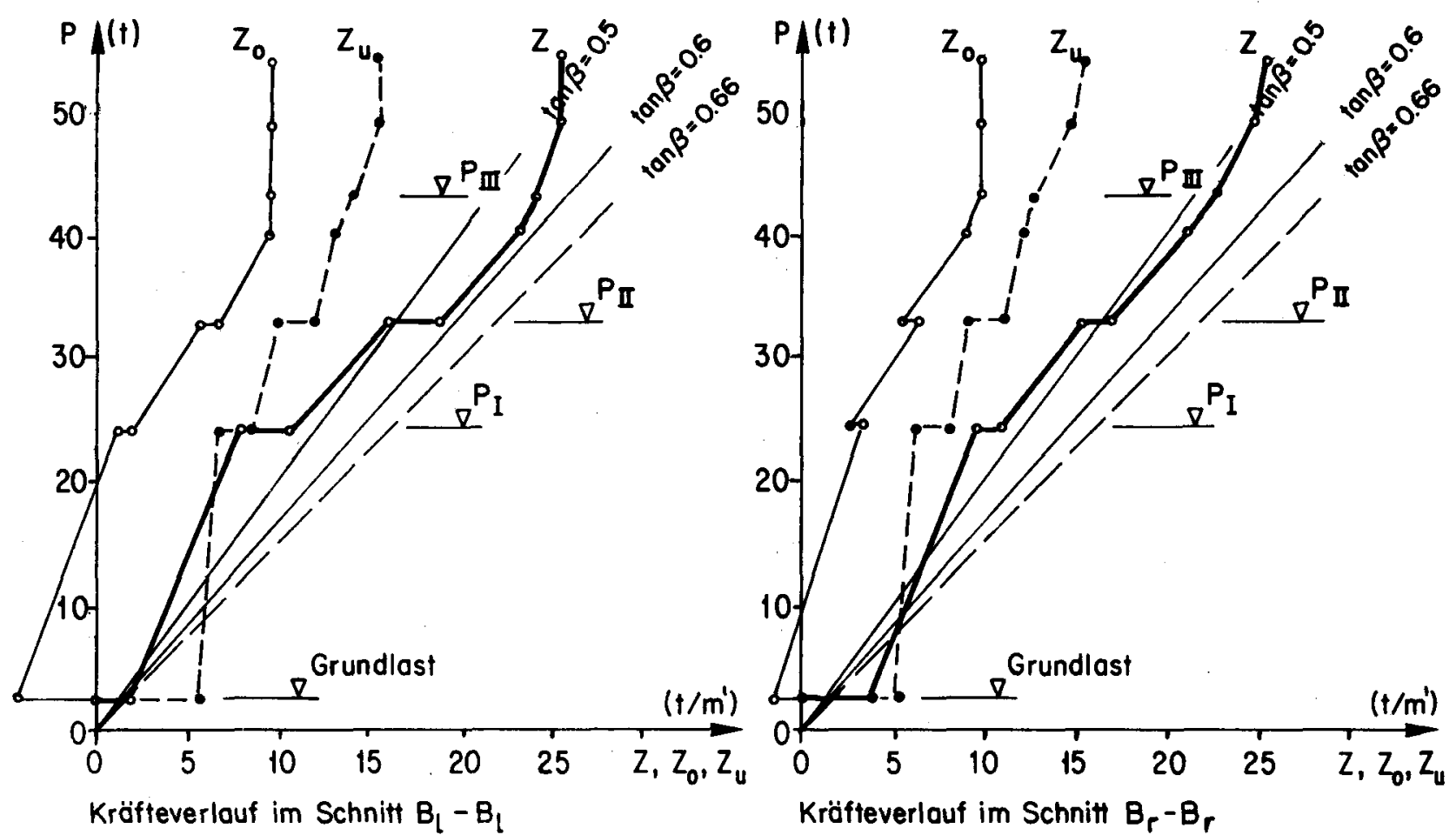

Bild 30-b: Kräfteverlauf in den Messstrecken $a-a$ beim Träger Z 6 


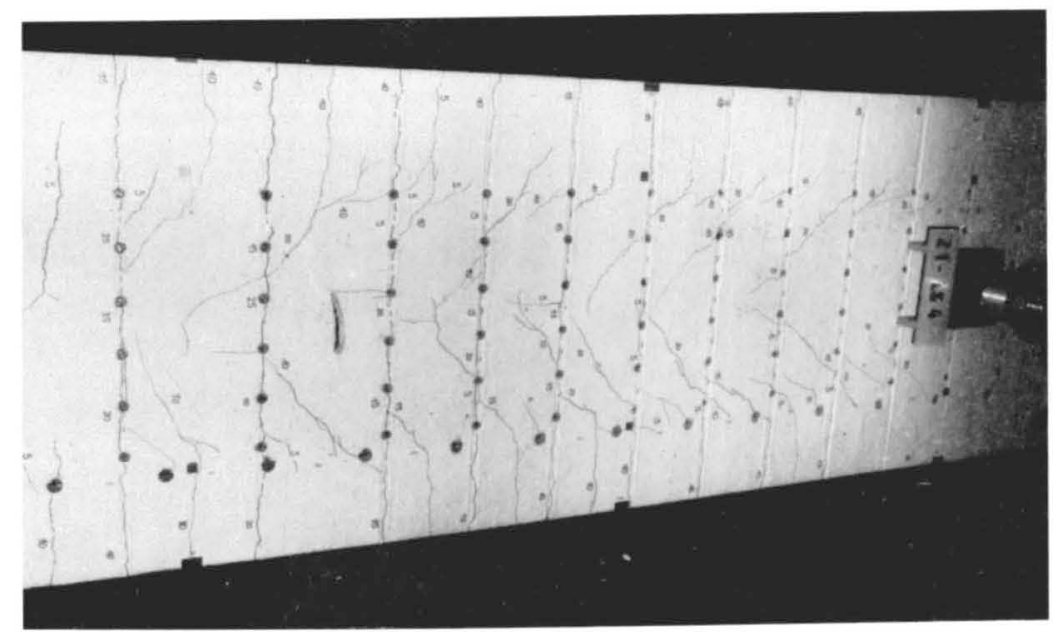

$P_{I}=24 t$
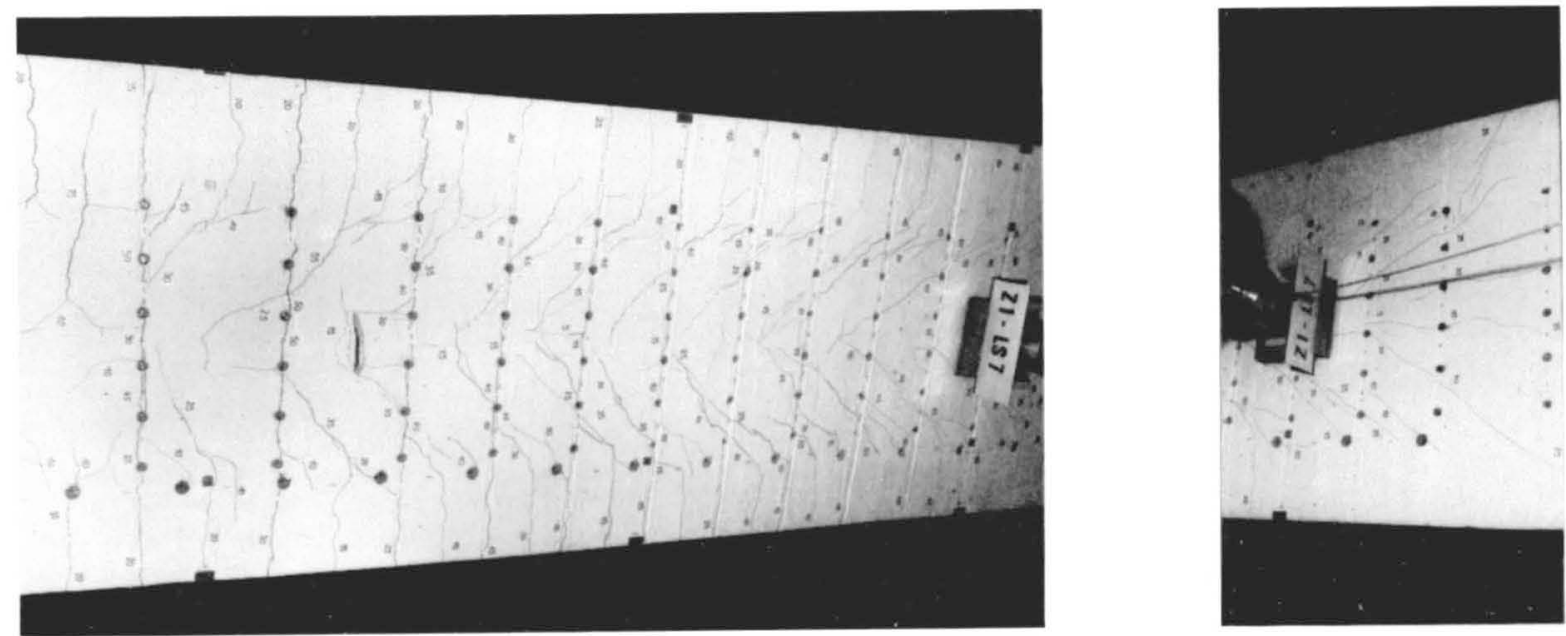

$P_{\text {III }}=43 \dagger$
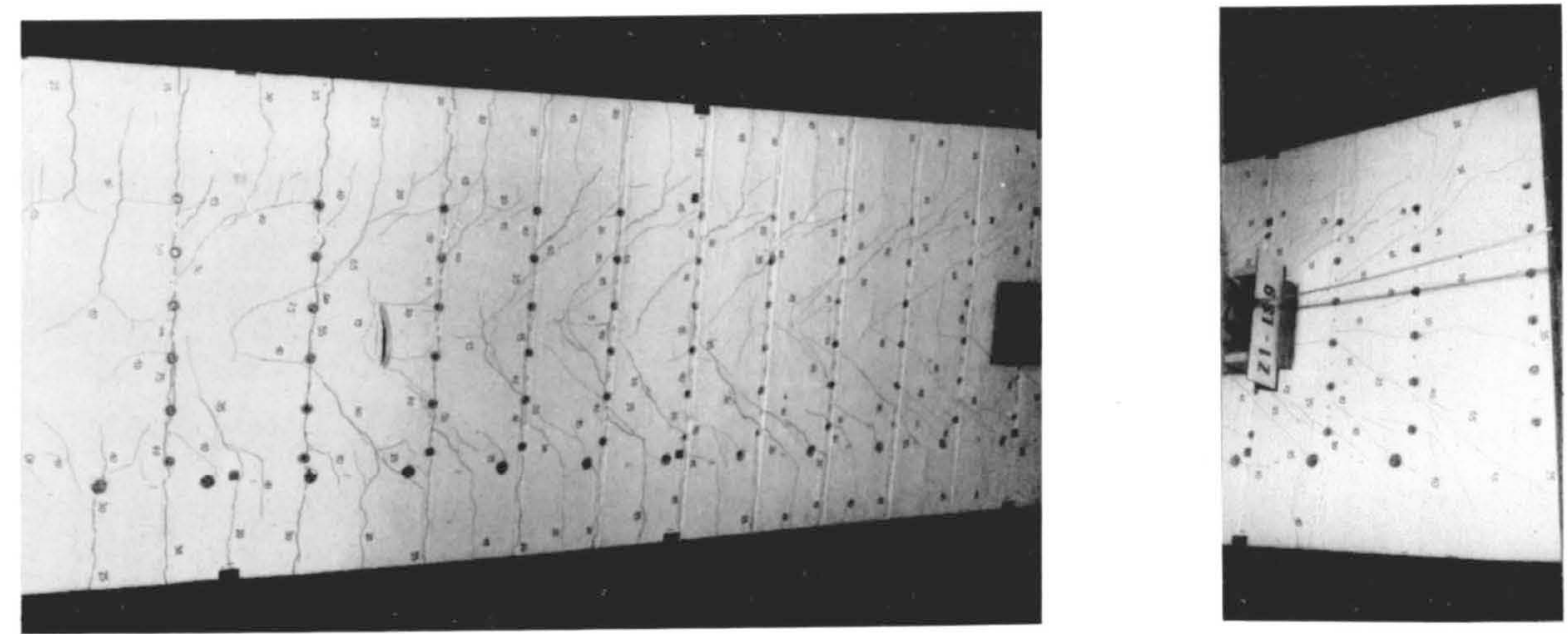

$P=54 t$

Bild 31: Rissebilder der Platte beim Träger Z1 (rechte Seite) 

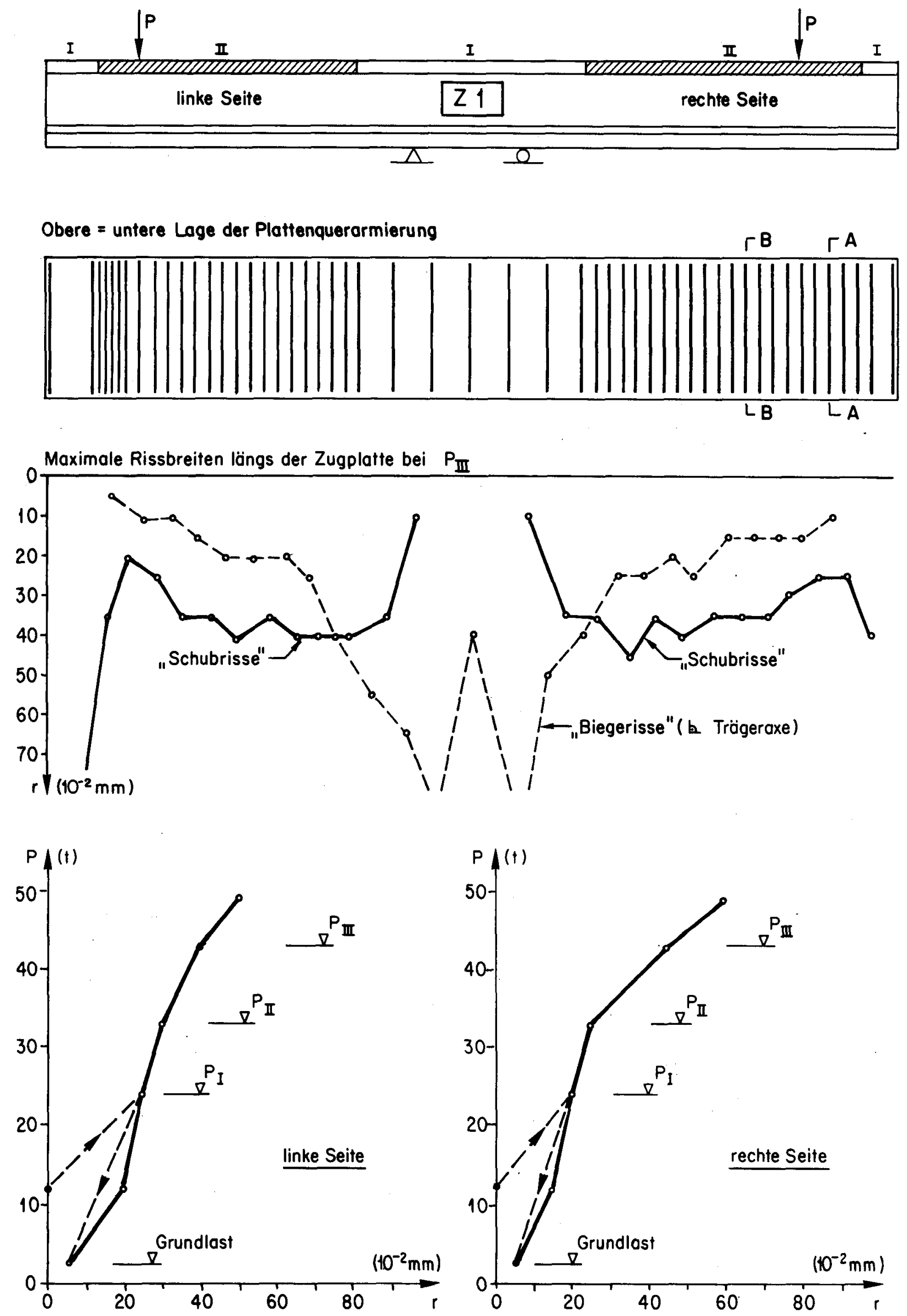

Verlauf der grössten Schubriss-Breiten im Bereich II

Bild 32: Rissbreiten auf der Plattenoberseite beim Träger Z 1 


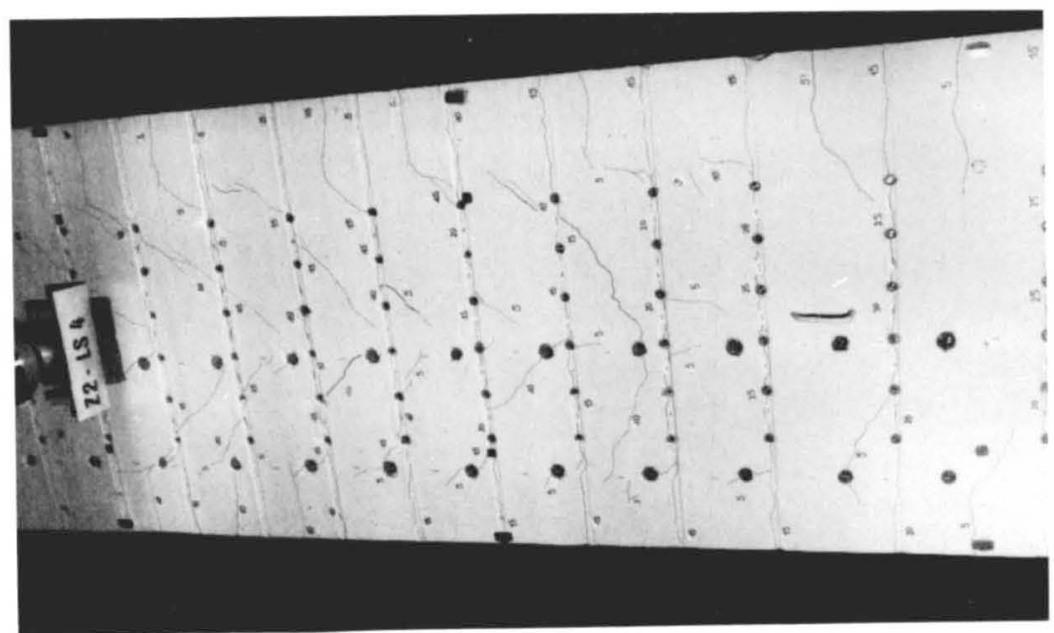

$P_{I}=24 \dagger$
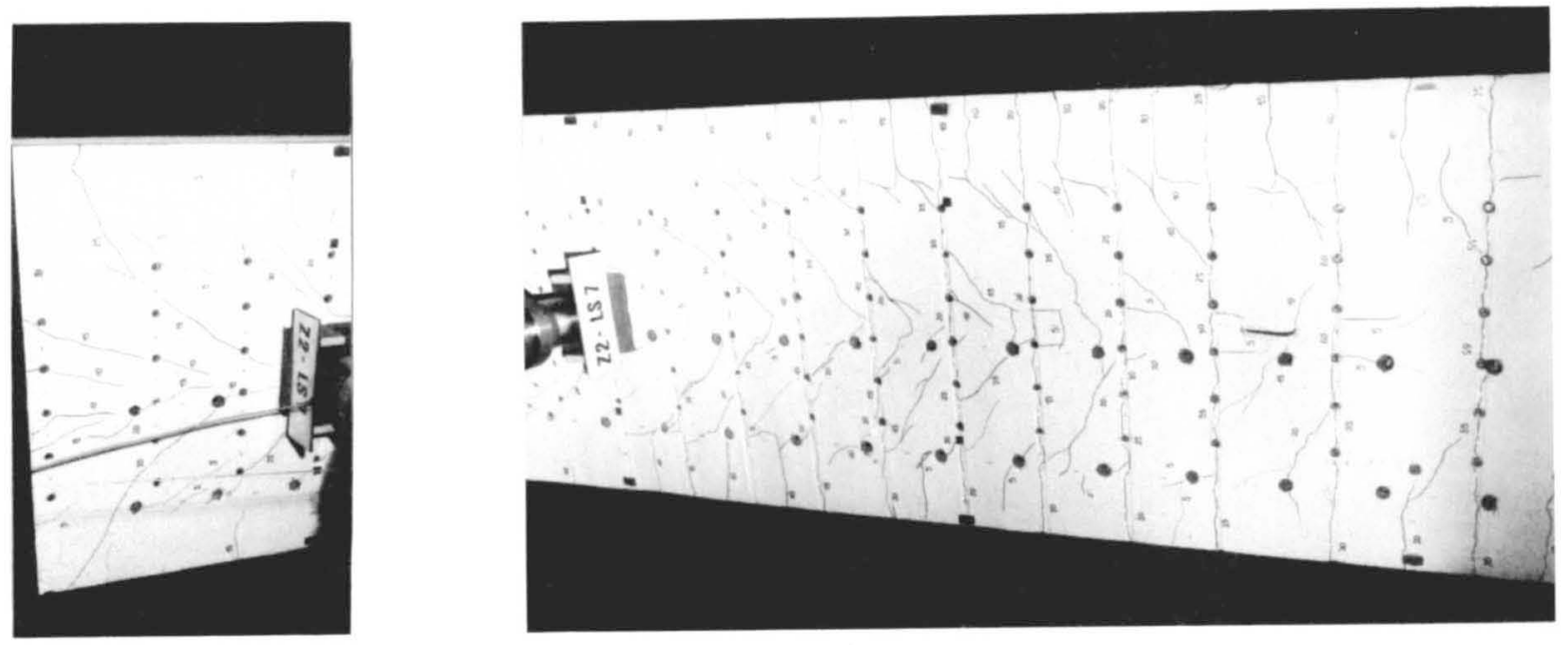

$P_{\text {III }}=43+$
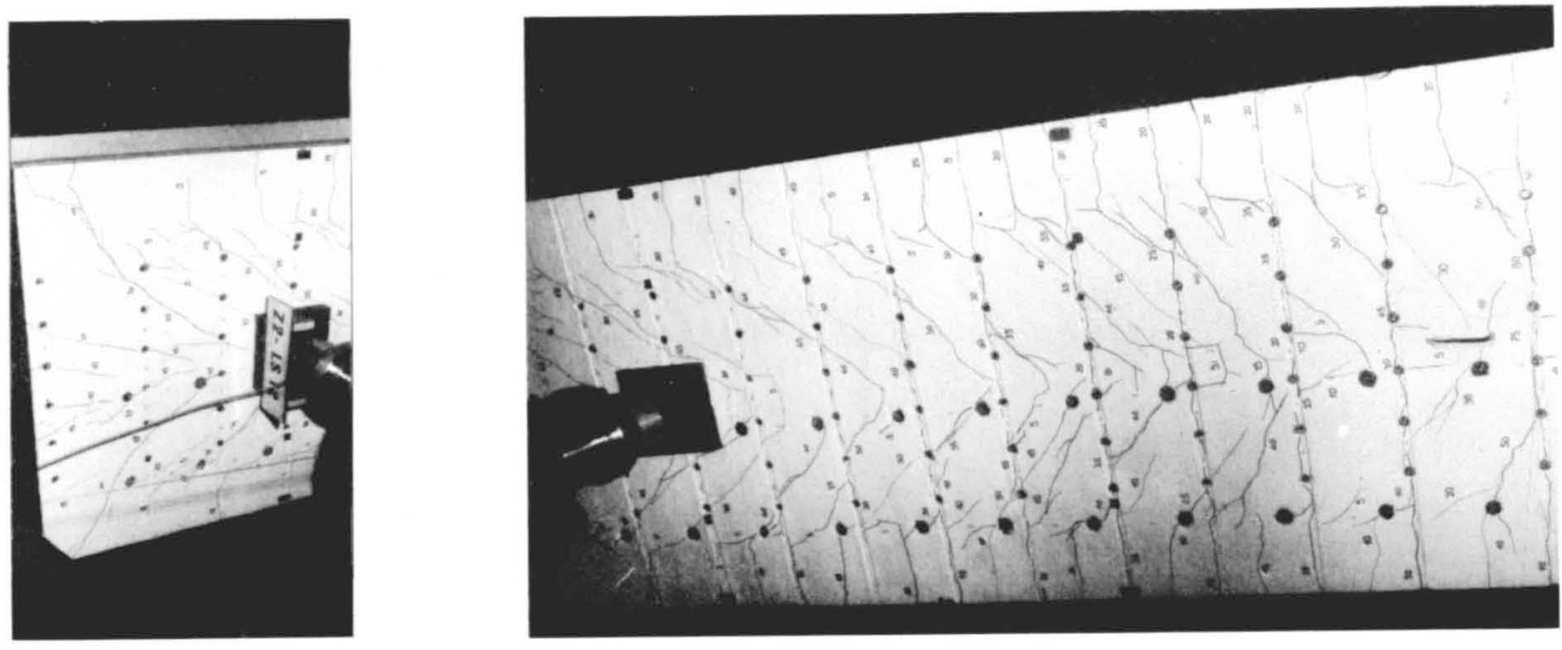

$P=56 \dagger$

Bild 33-a: Rissebilder der Platte beim Träger Z 2 (linke Seite) 


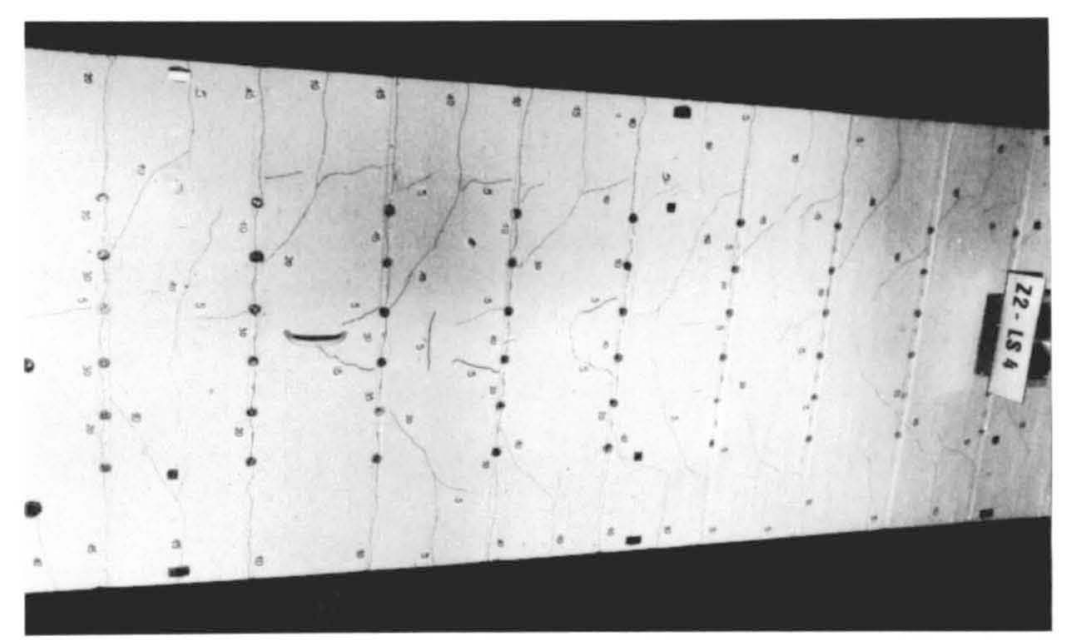

$P_{I}=24 \dagger$
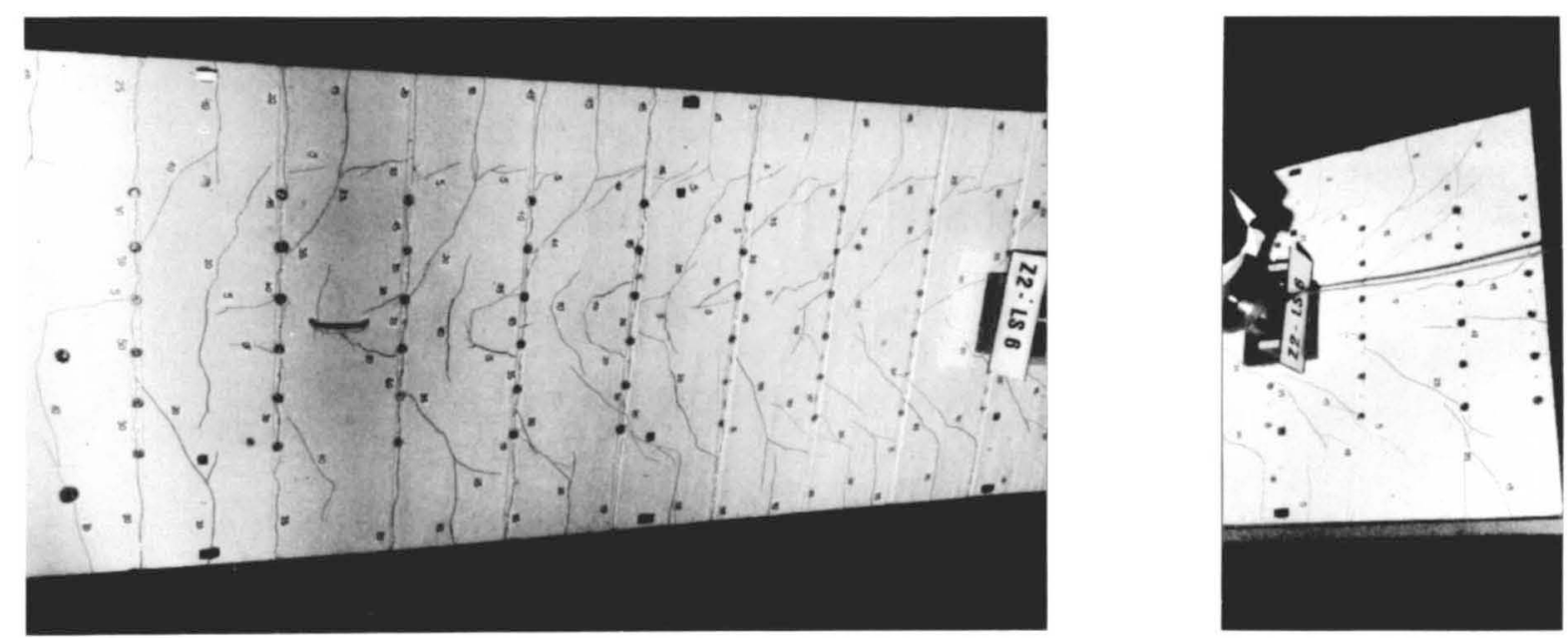

$P_{\text {III }}=43 \dagger$
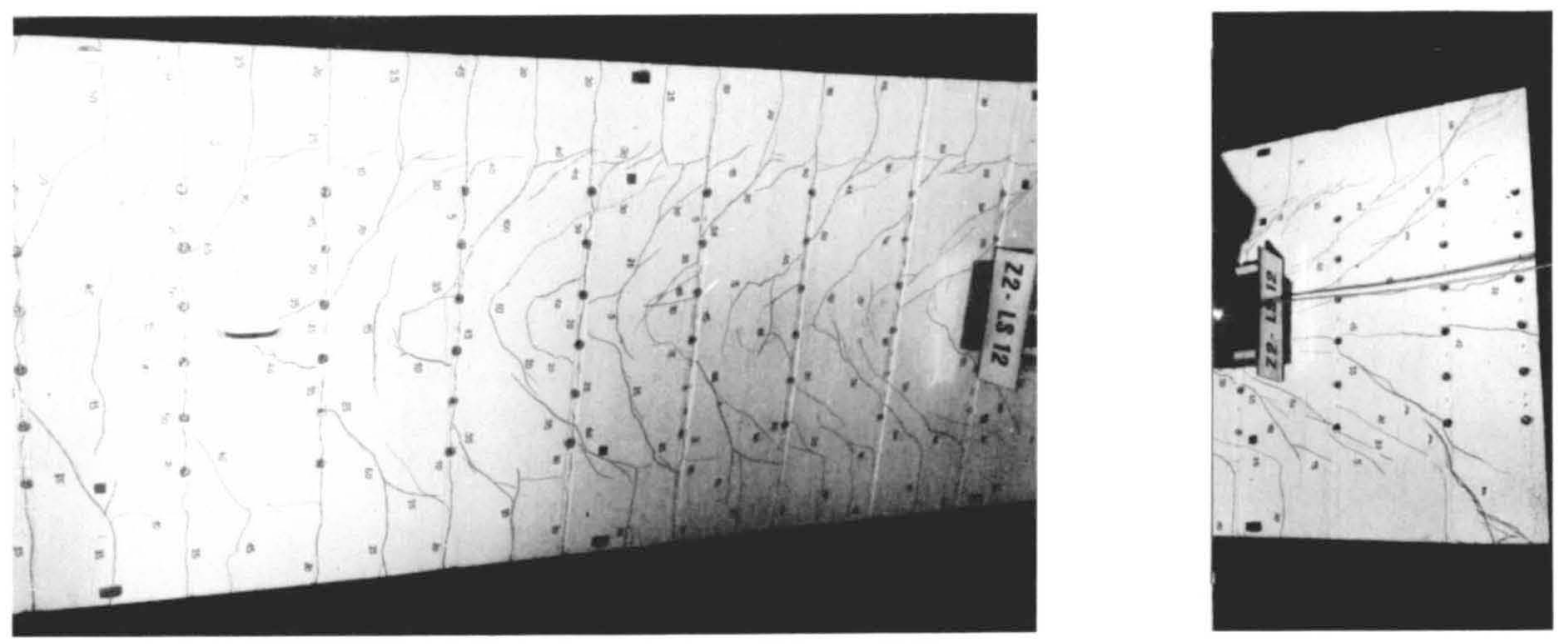

$P=54 t$

Bild 33-b: Rissebilder der Platte beim Träger Z2 (rechte Seite) 

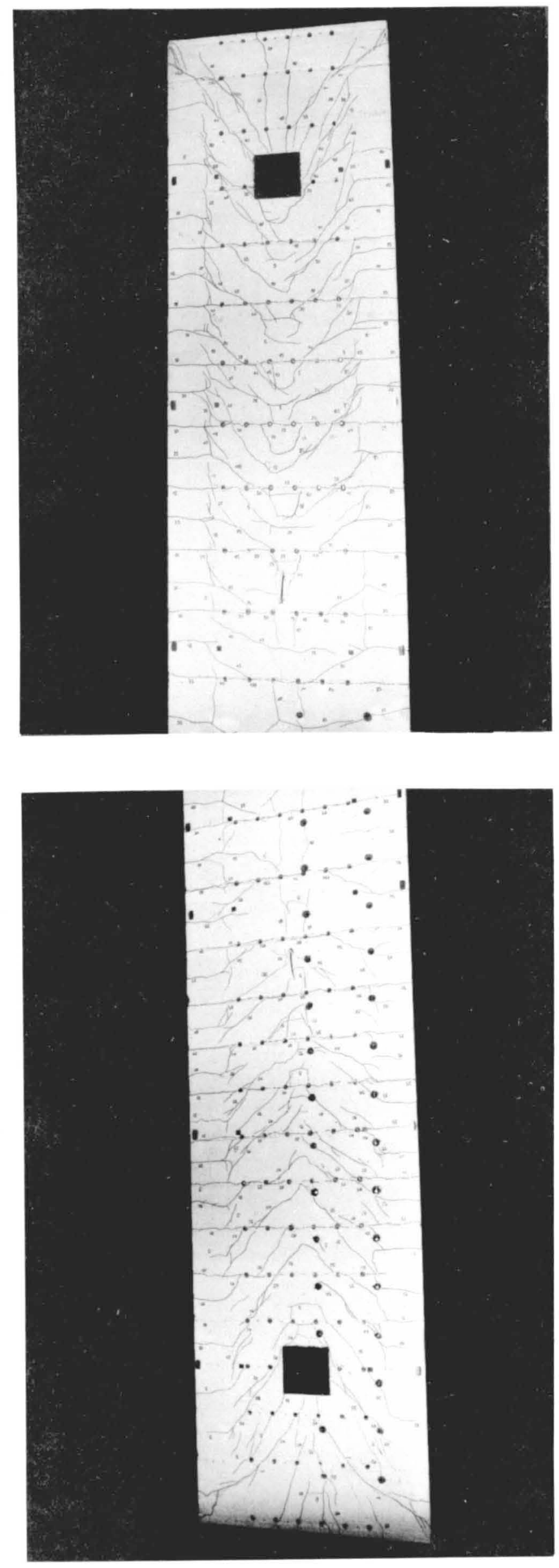

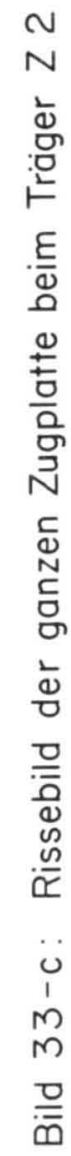



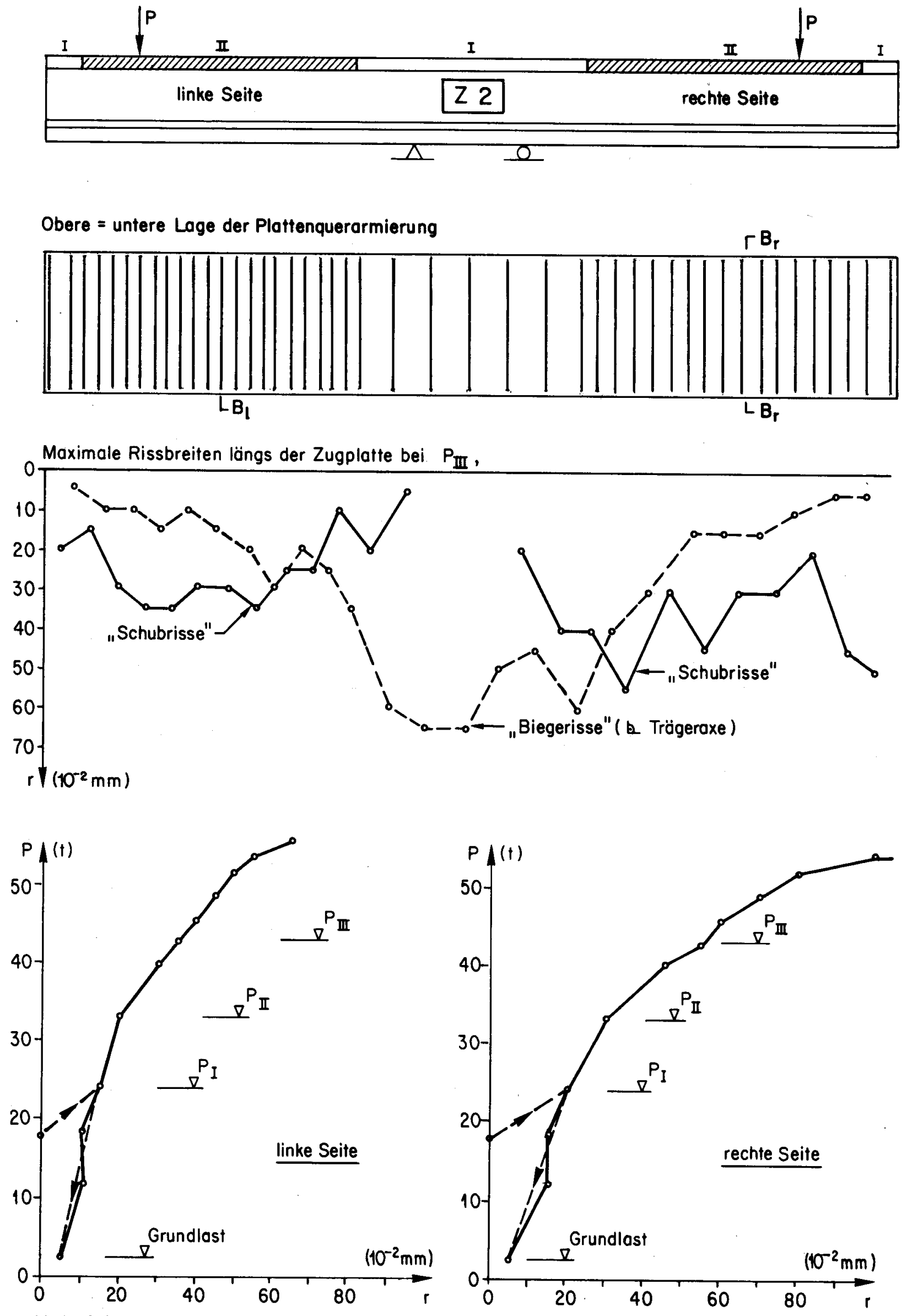

Verlauf der grössten Scuhubriss - Breiten im Bereich II

Bild 34: Rissbreiten auf der Plattenoberseite beim Träger Z 2 


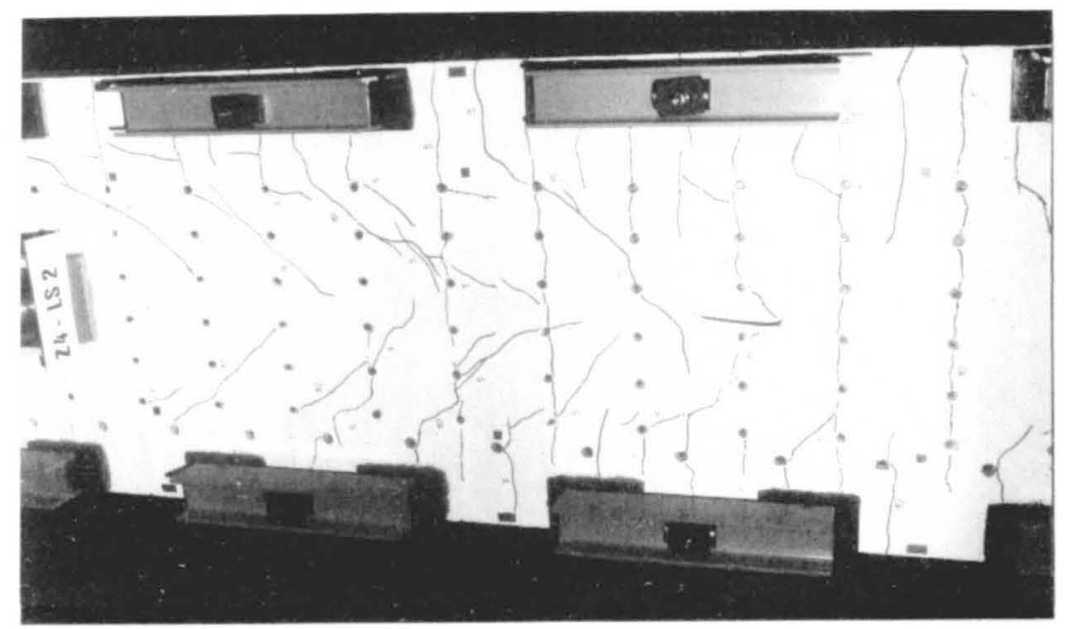

$$
P_{I}=24 \dagger, \bar{P}_{0}
$$
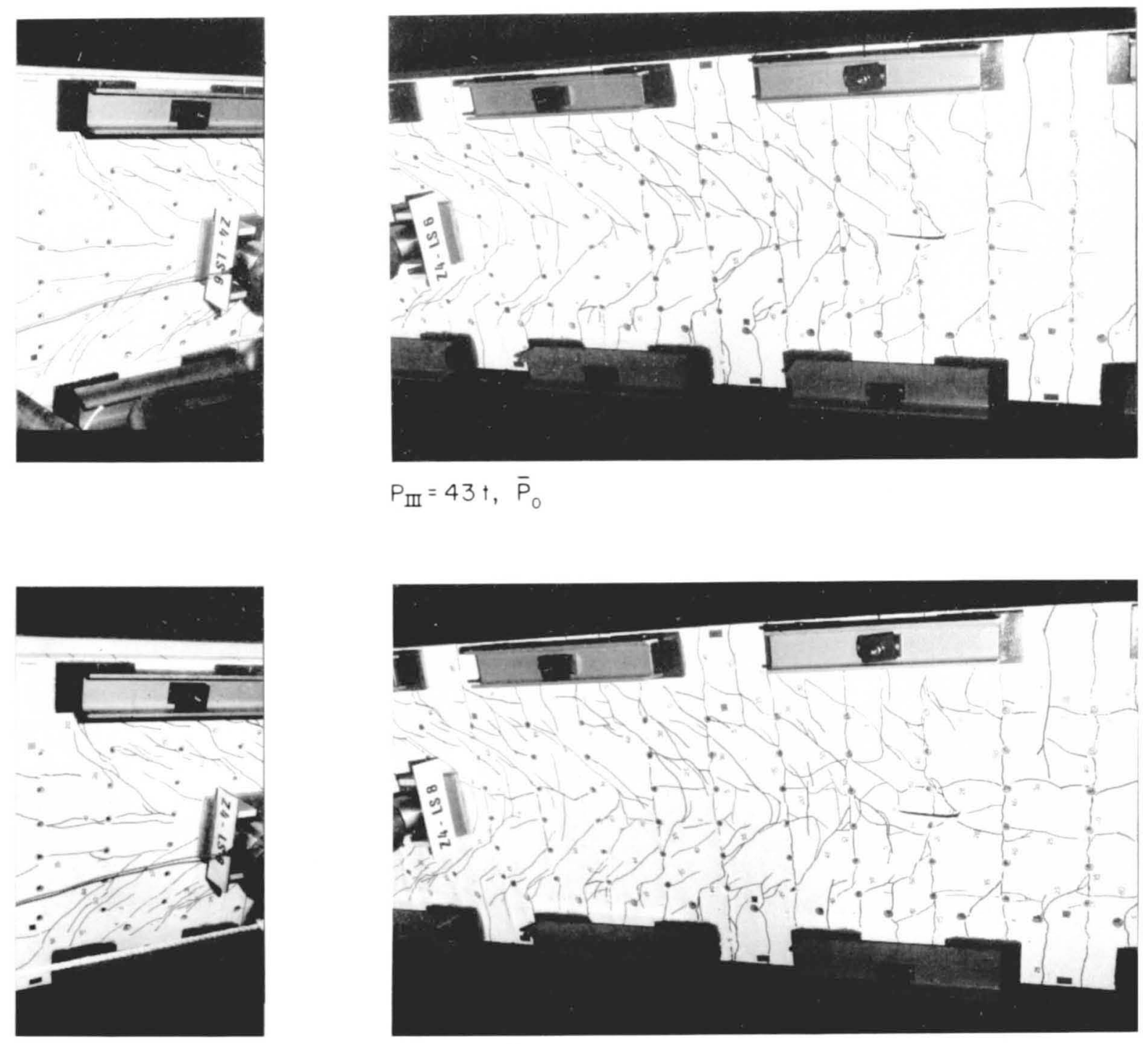

$P_{\text {III }}=43 t, \quad \bar{P}_{I}=3.90 \dagger$

Bild 35-a: Rissebilder der Platte beim Träger Z 4 (linke Seite) 


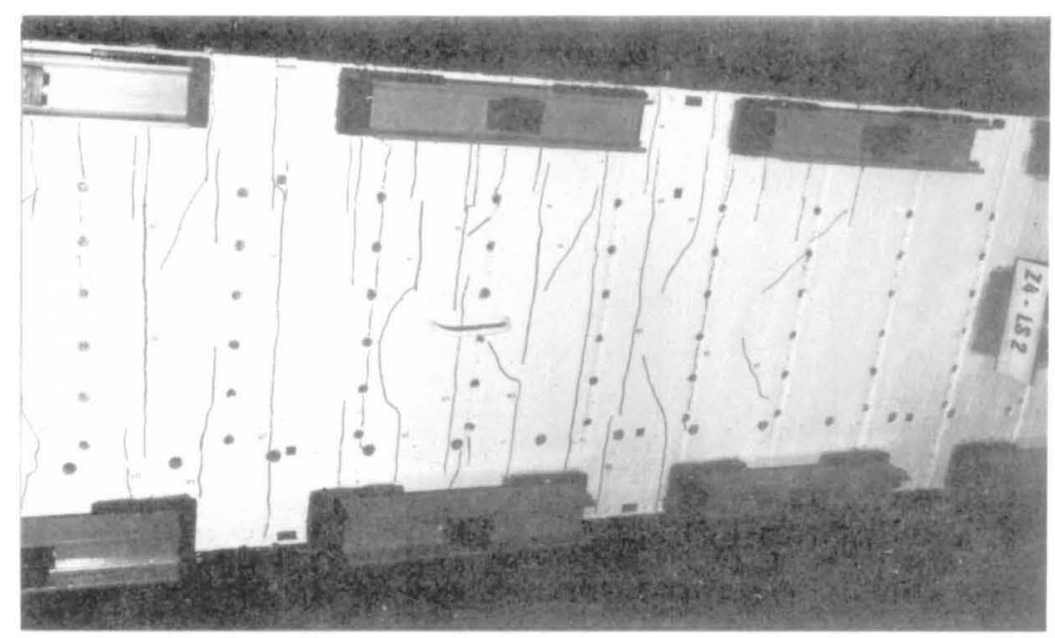

$P_{I}=24 \dagger$
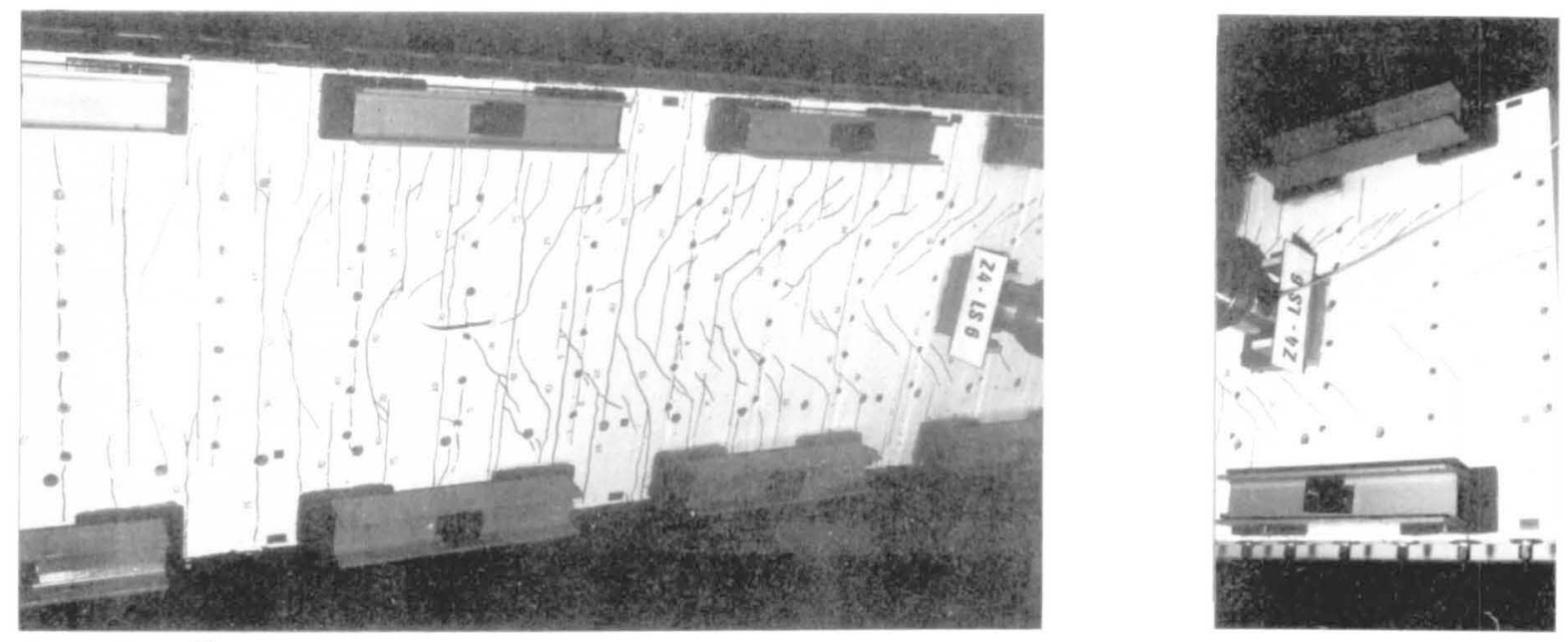

$P_{\text {III }}=43 t, \bar{P}_{0}$
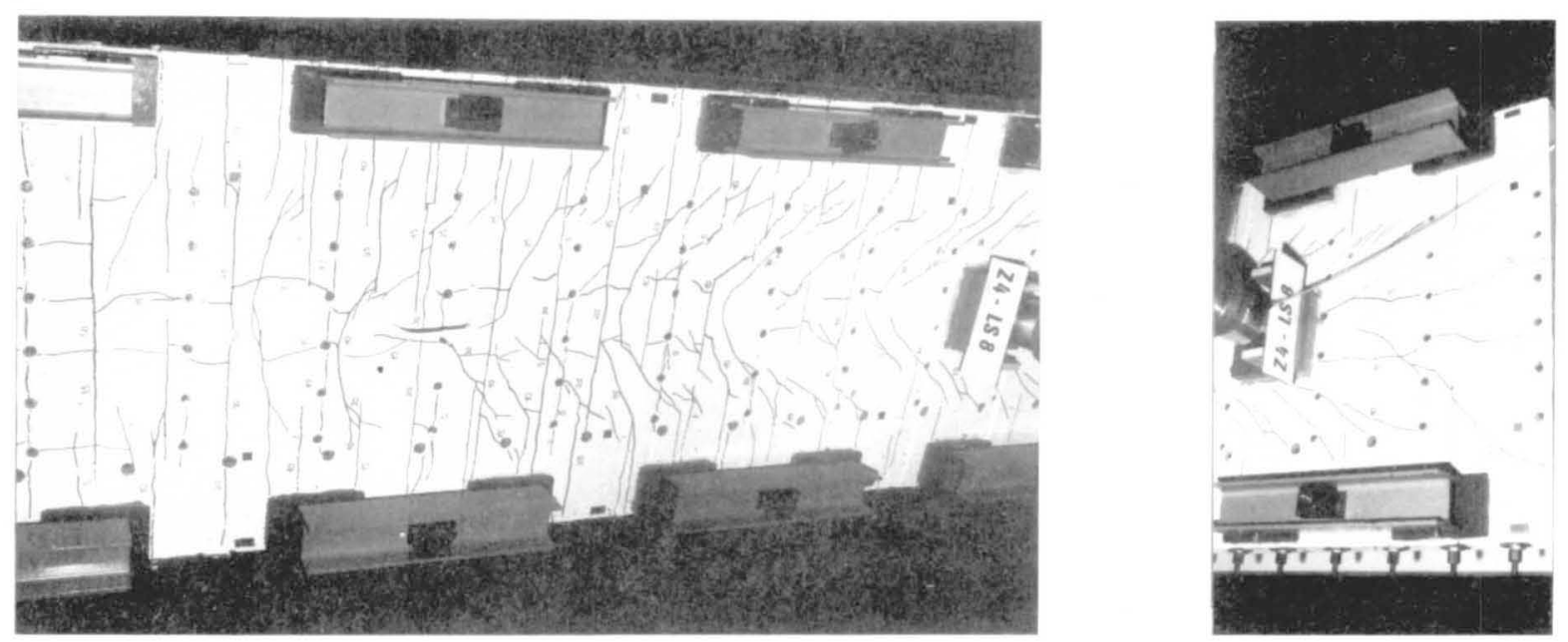

$P_{\text {II }}=43 \uparrow, \bar{P}_{I}=3.90 \dagger$

Bild 35-b: Rissebilder der Platte beim Träger Z 4 (rechte Seite) 


\begin{tabular}{|l|l|l|l|l|}
\hline III & II & II \\
\hline linke Seite: schlaff armiert & $\mathrm{Z} 4$ & rechte Seite: teilweise vorgespannt \\
\hline
\end{tabular}

10

Obere Loge der Plattenquerormierung

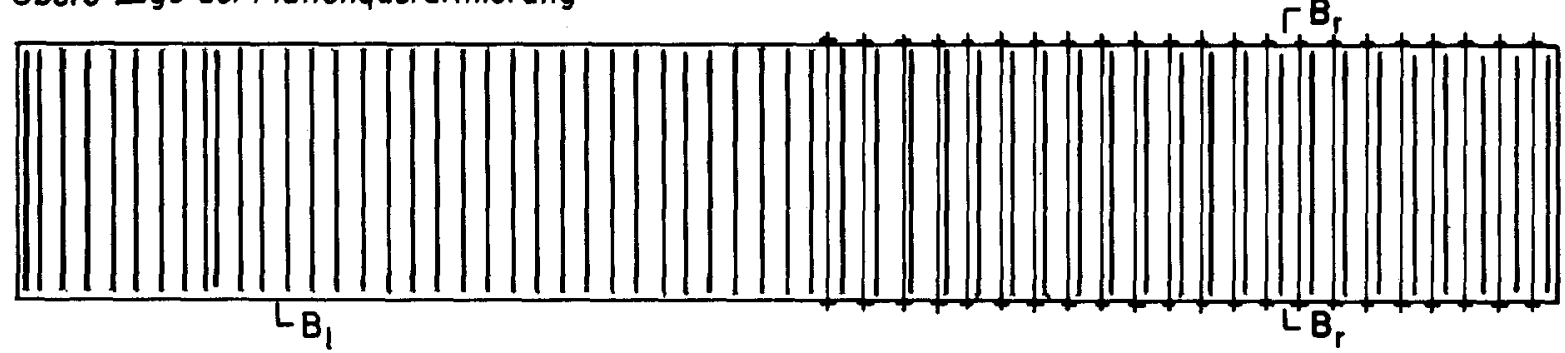

Maximale Rissbreiten längs der Zugplatte bei $P_{\text {III }}, \bar{P}_{0}$
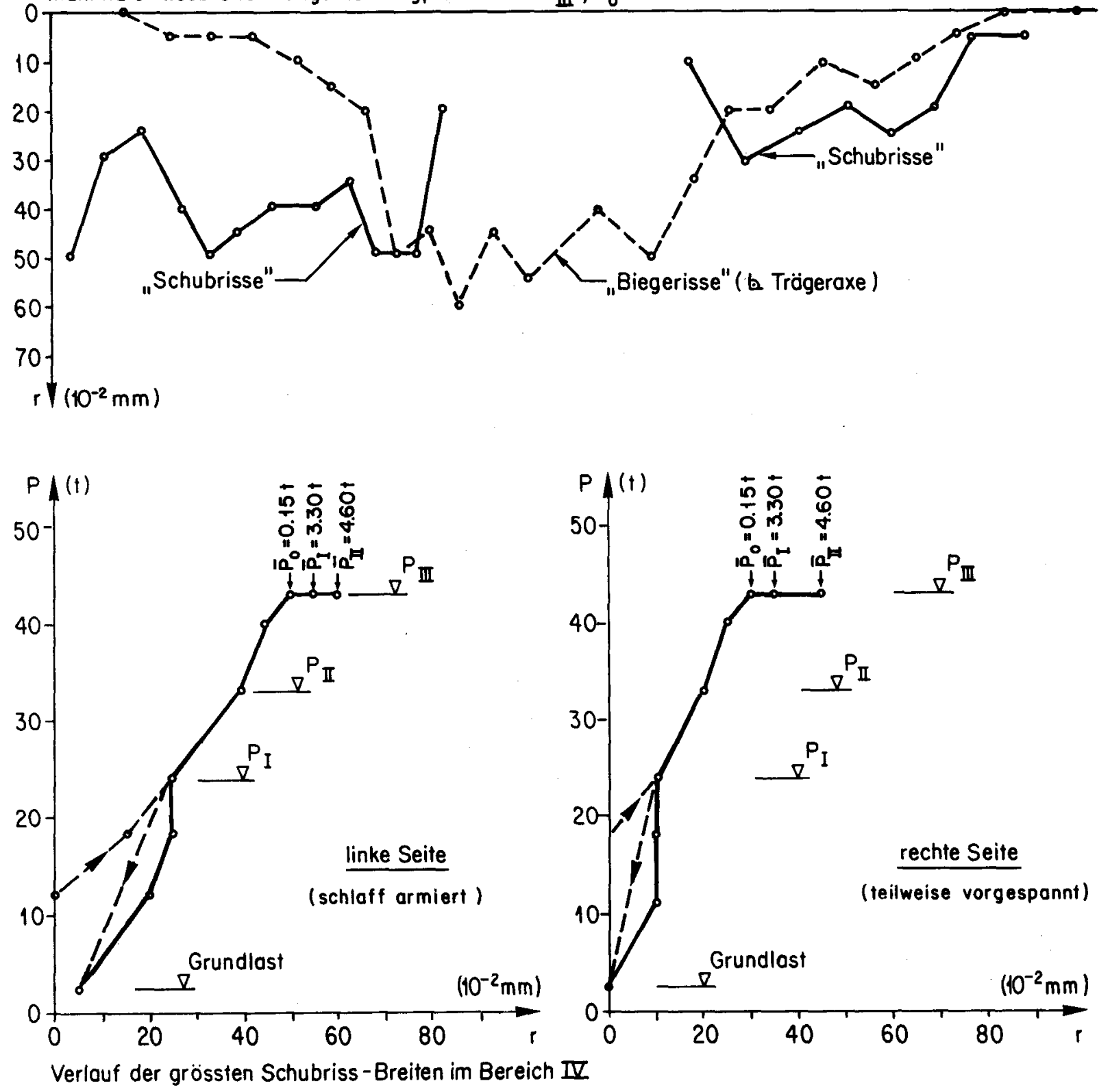

Bild 36: Rissbreiten auf der Plattenoberseite beim Träger Z 4 


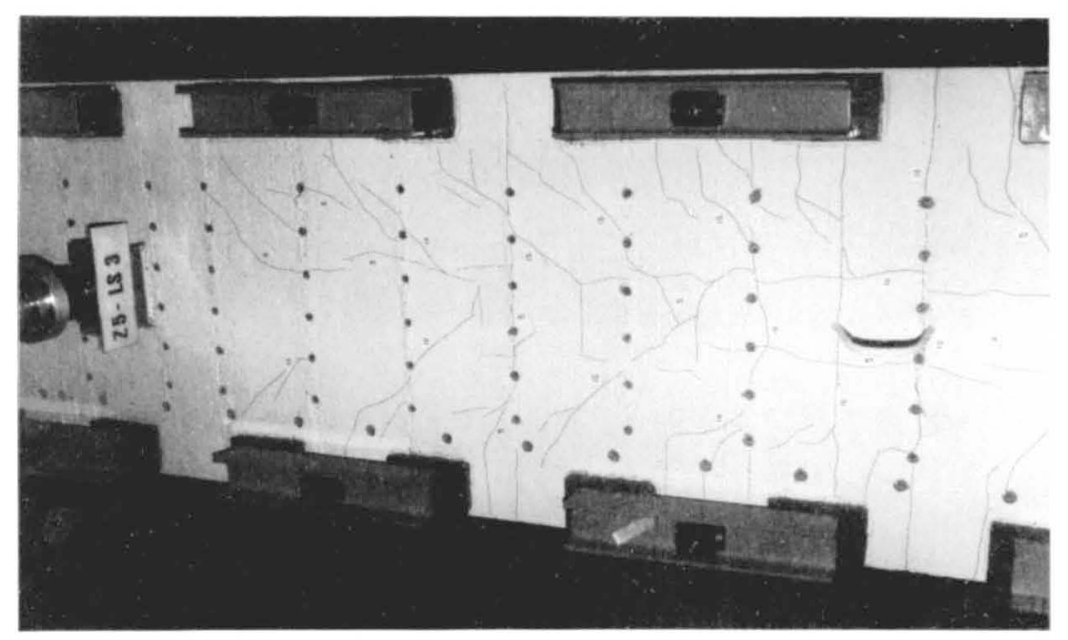

$P_{I}=24 \dagger, \bar{P}_{I}=2.15 \dagger$
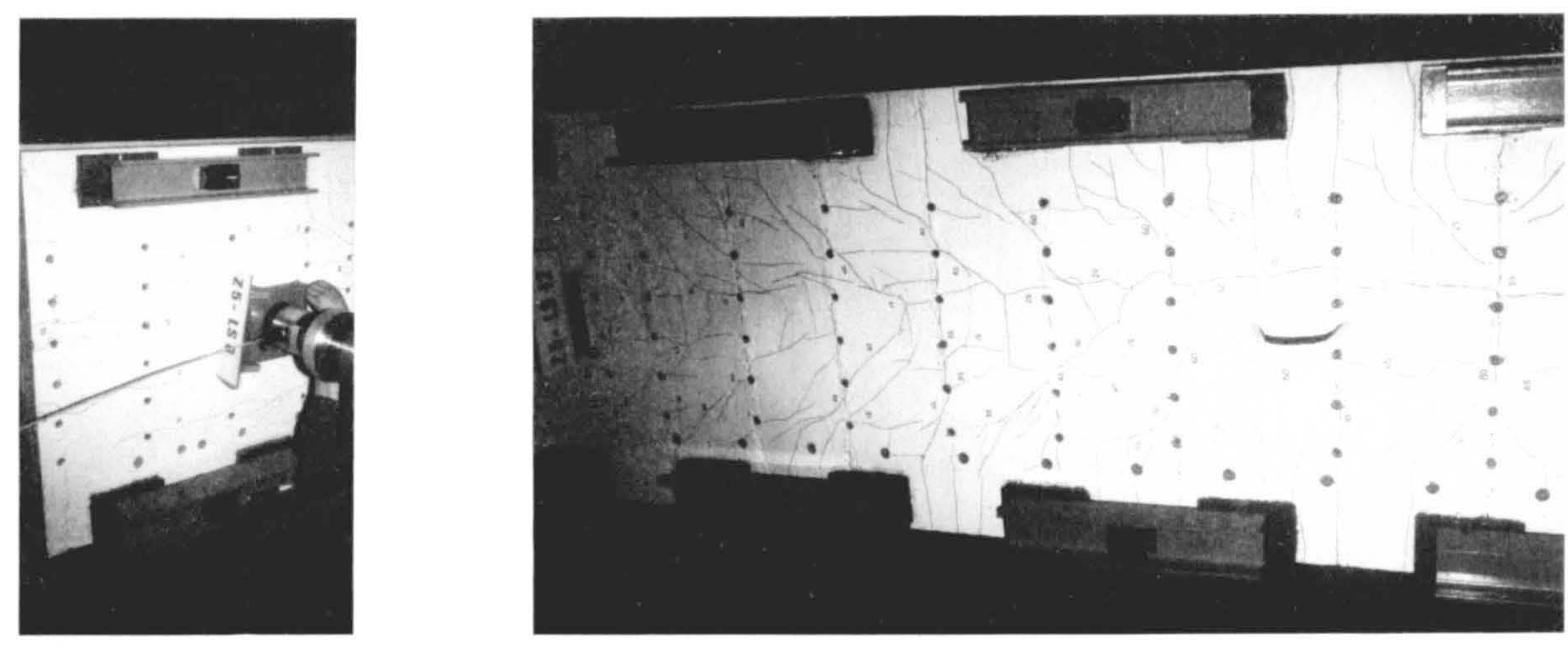

$P_{\text {III }}=43 \dagger, \bar{P}_{\text {II }}=3.90 \dagger$
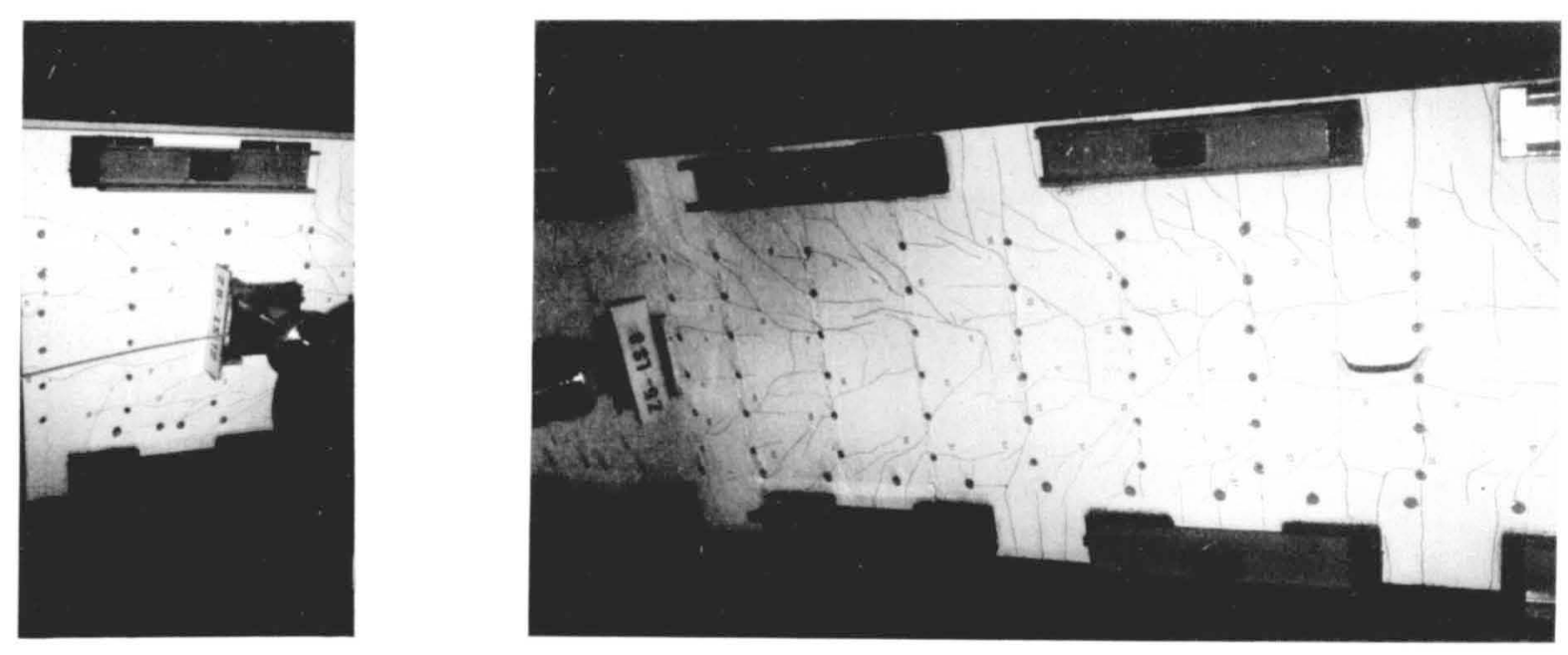

$P=541, \bar{P}_{\text {II }}=3.901$

Bild 37-a: Rissebilder der Platte beim Träger Z 5 (linke Seite) 


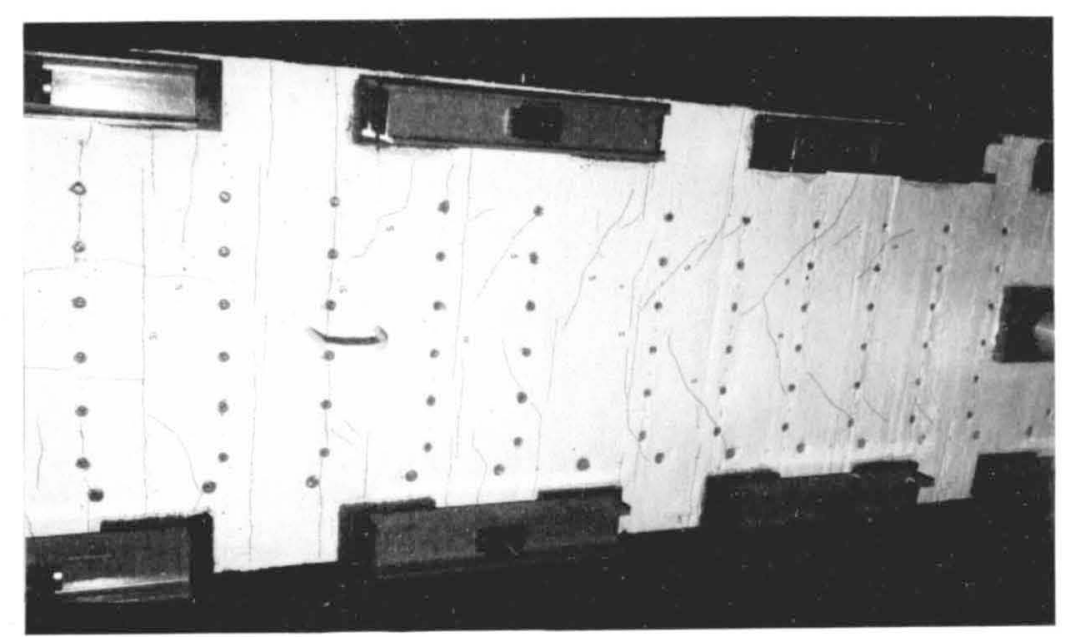

$P_{I}=24 t, \bar{P}_{I}=2.15 t$
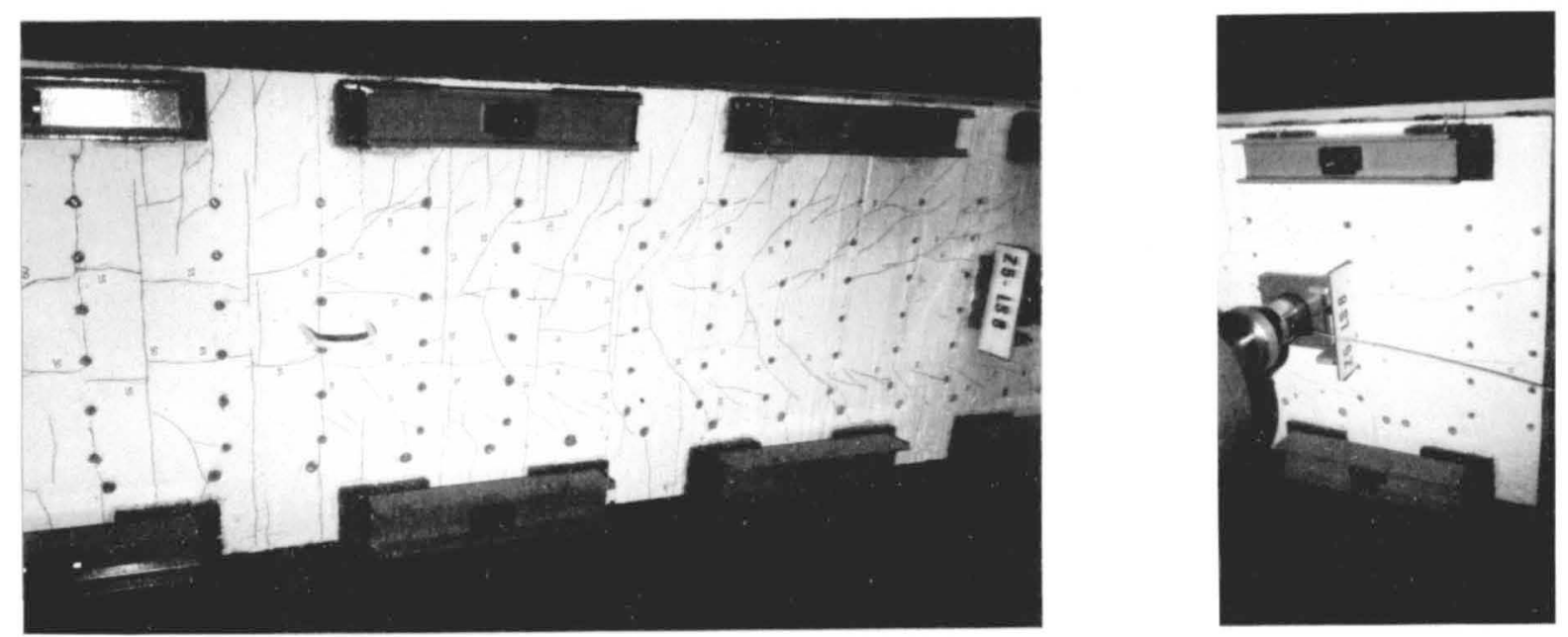

$P_{\text {III }}=43 t, \bar{P}_{\text {III }}=3.90 t$
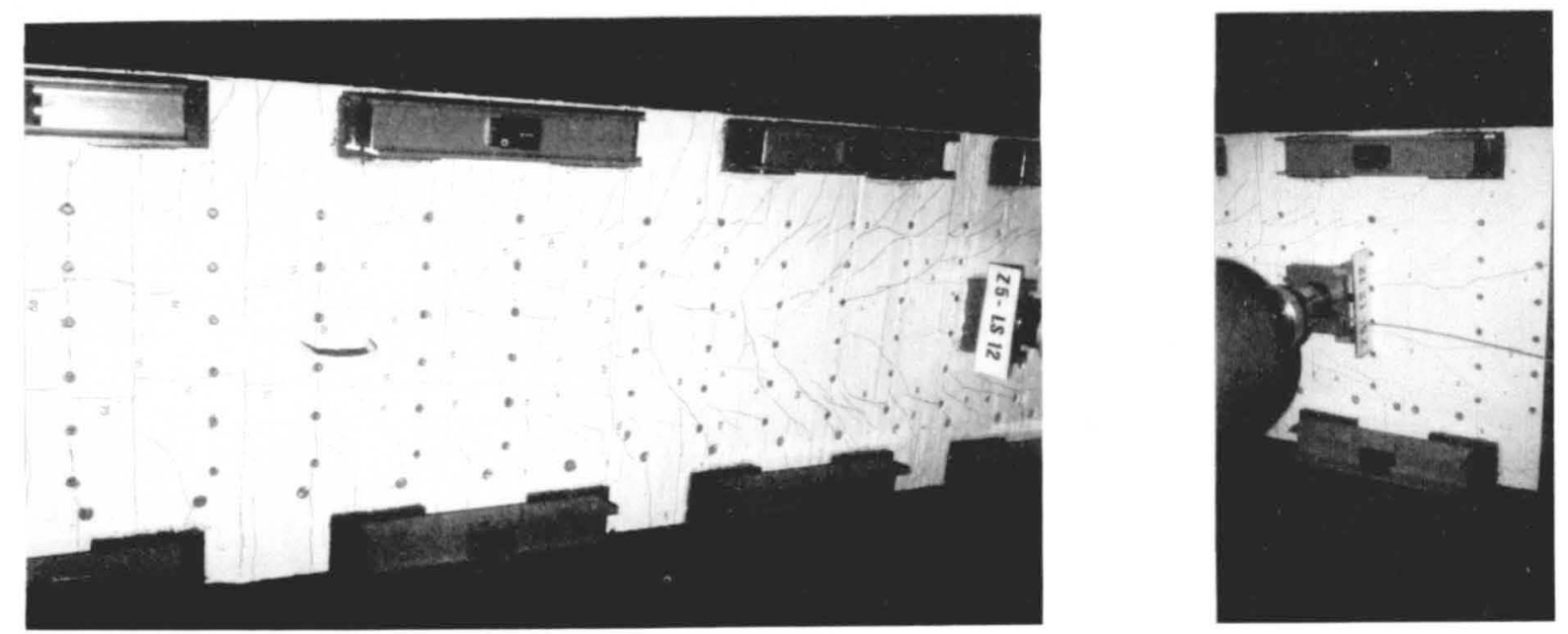

$P=541, \bar{P}_{\text {III }}=3.90 \dagger$

Bild 37-b: Rissebilder der Platte beim Träger Z 5 (rechte Seite) 

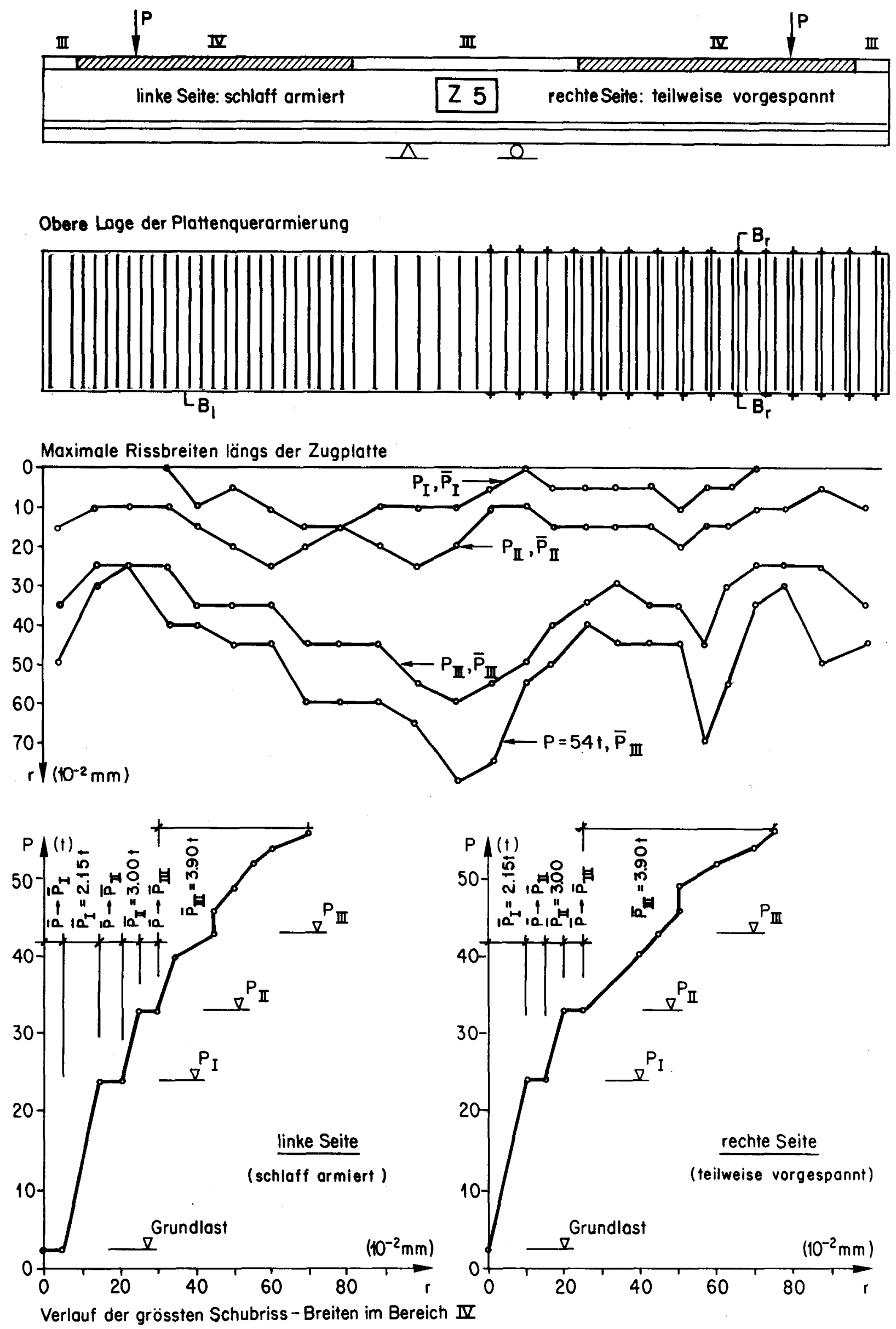

Bild 38: Rissbreiten auf der Plattenoberseite beim Träger Z 5 


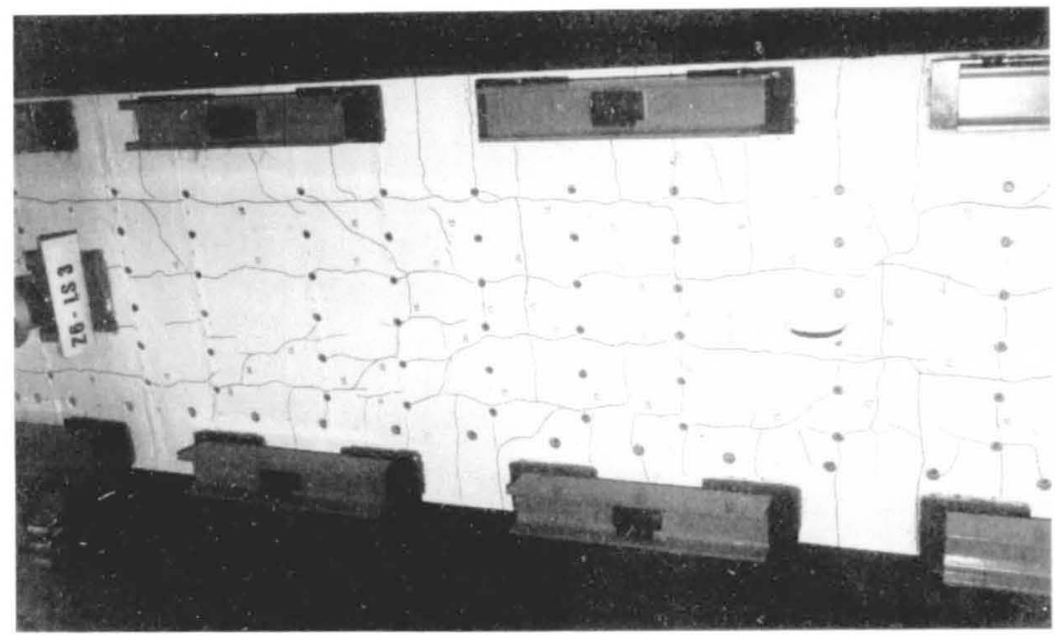

$P_{I}=24 \dagger, \bar{P}_{I}=2.15 \dagger$
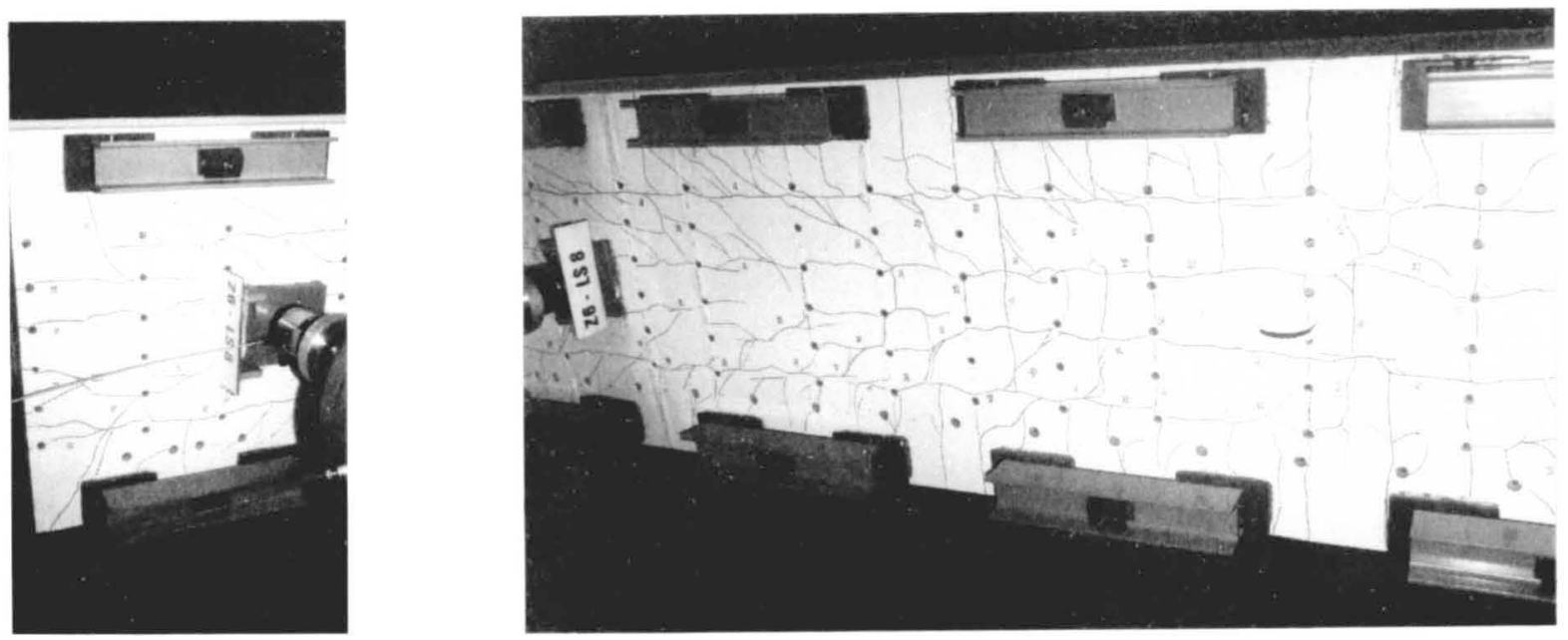

$P_{\text {III }}=43 \dagger, \bar{P}_{\text {II }}=3.90 \dagger$
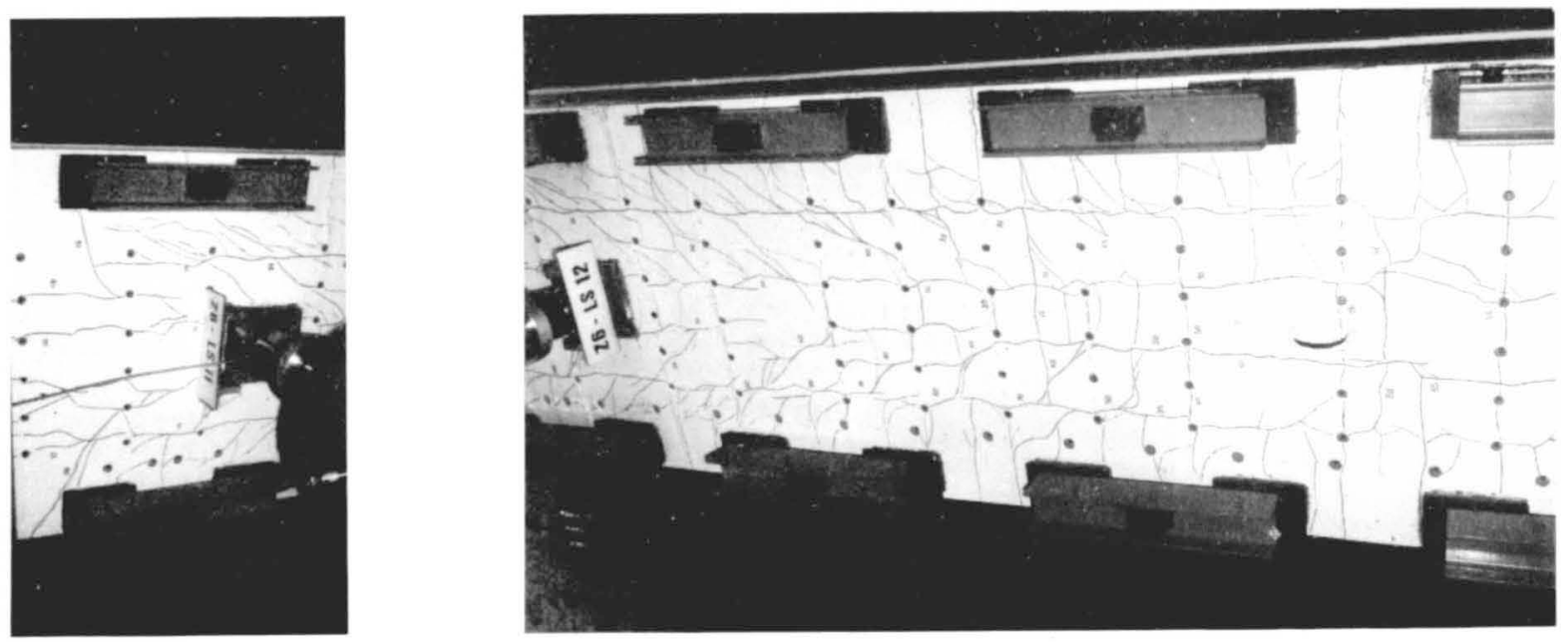

$P=54 t, \bar{P}_{\text {II }}=3.90 \dagger$

Bild 39-a: Rissebilder der Platte beim Träger Z6 (linke Seite) 


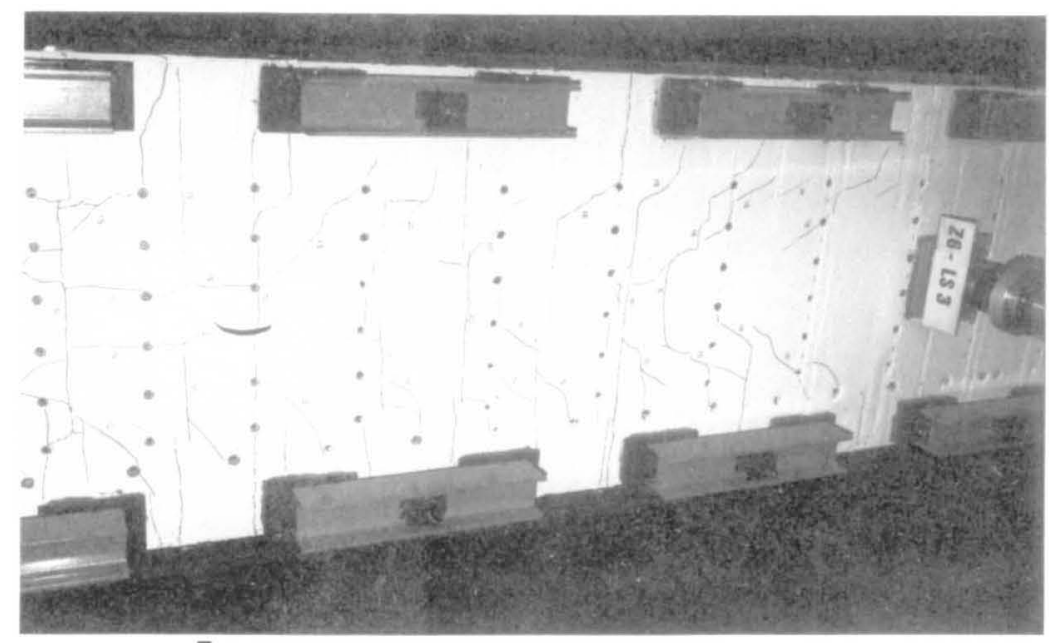

$P_{I}=24 \uparrow, \bar{P}_{I}=2.15 \dagger$
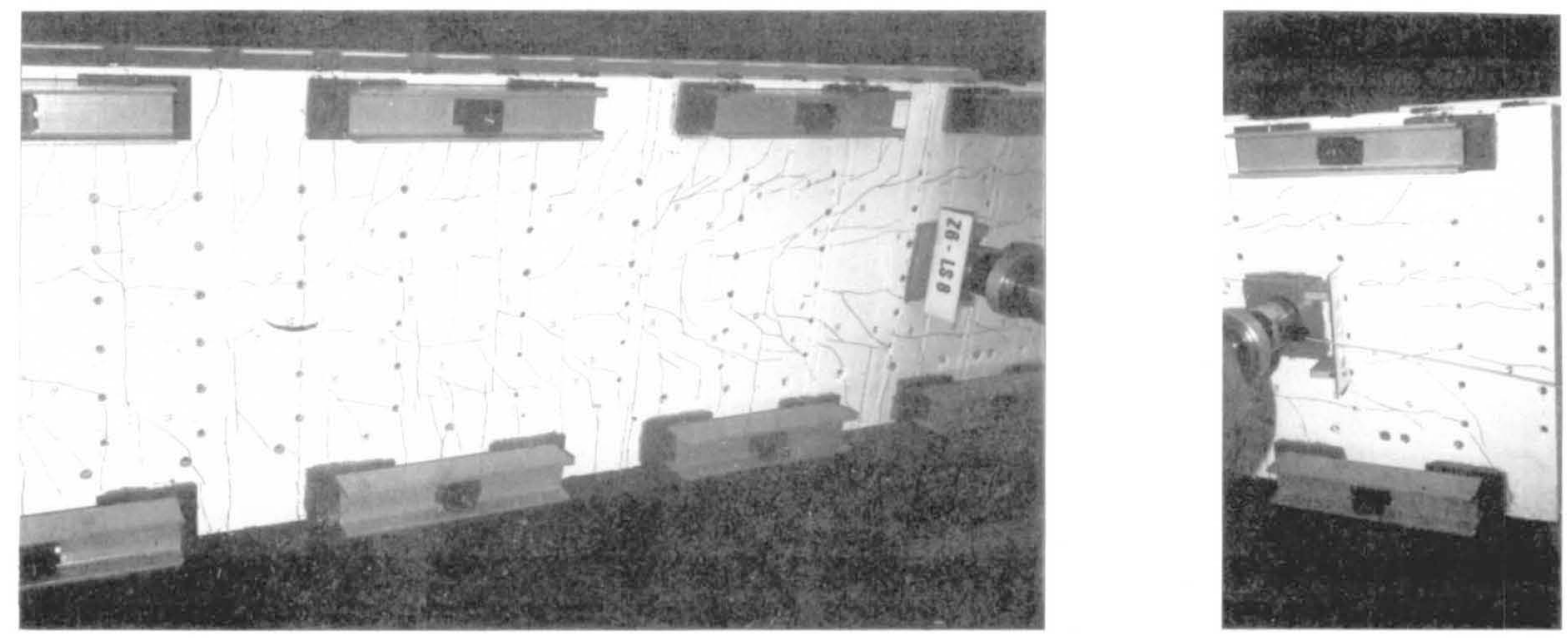

$P_{\text {III }}=43 \uparrow, \bar{P}_{\text {III }}=3.90 \dagger$
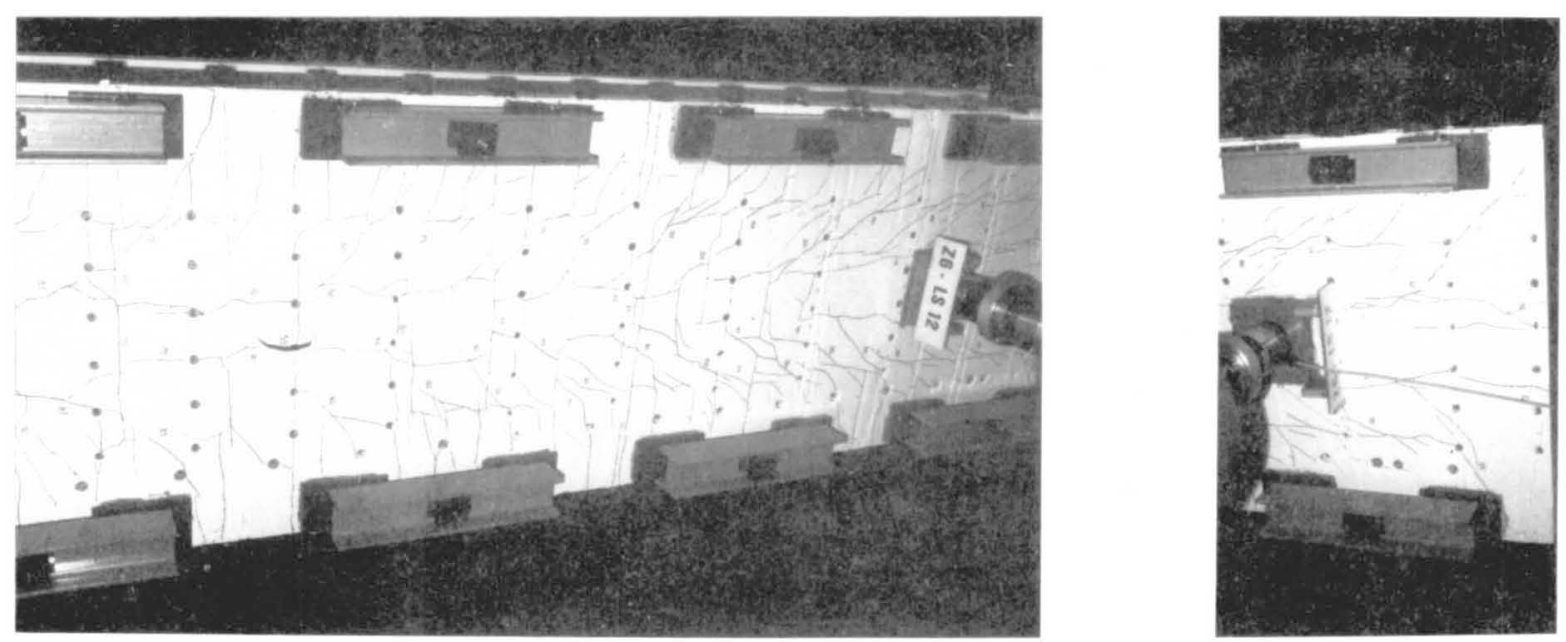

$P=54 \uparrow, \bar{P}_{\text {III }}=3.90 \dagger$

Bild 39-b: Rissebilder der Platte beim Träger Z 6 (rechte Seite) 


\begin{tabular}{|l|l|l|l|}
\hline III & II & II & II \\
\hline linke Seite: schlaff armiert & $\mathrm{Z}$ & II \\
\hline
\end{tabular}

10
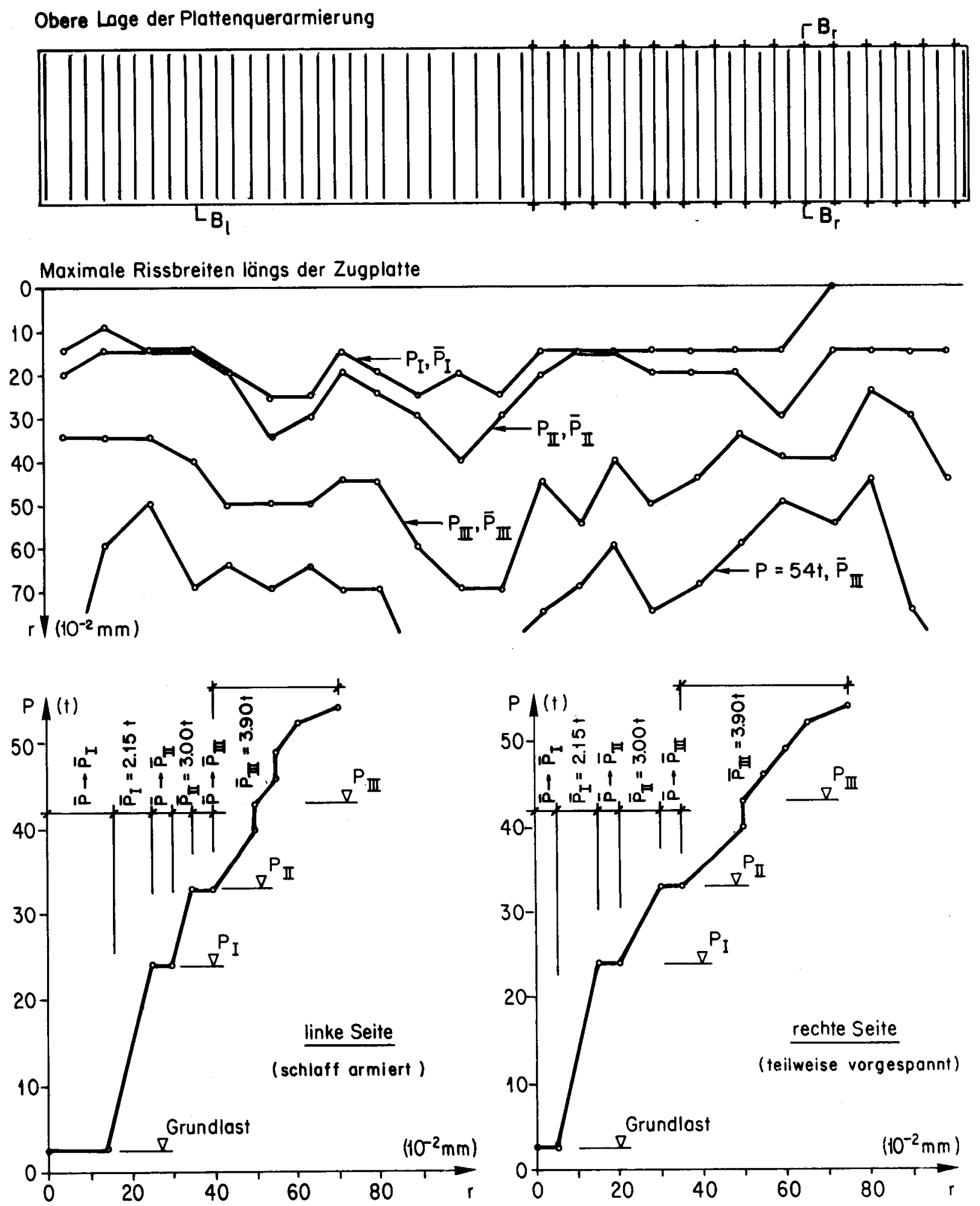

Verlouf der grössten Schubriss-Breiten im Bereich IV

Bild 40: Rissbreiten auf der Plattenoberseite beim Träger Z 6 


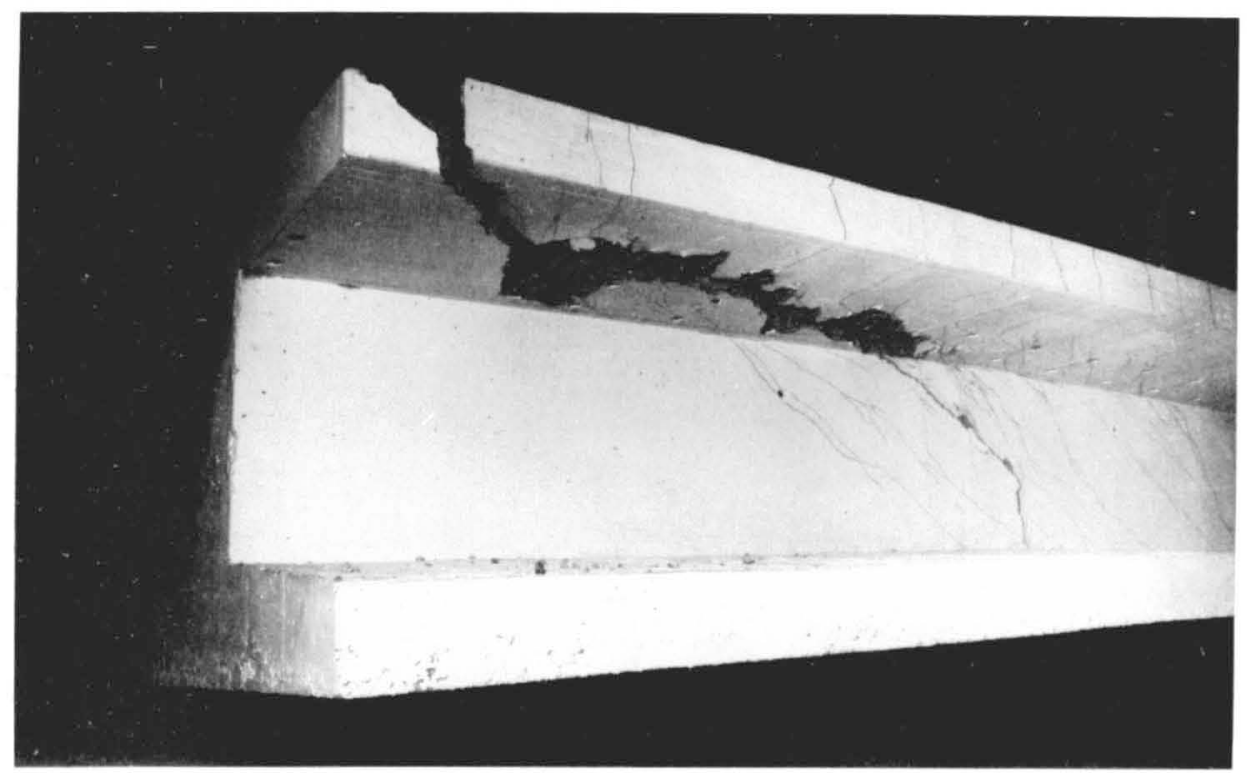

Ansicht von unten $\left(P_{u}=52 \dagger\right)$

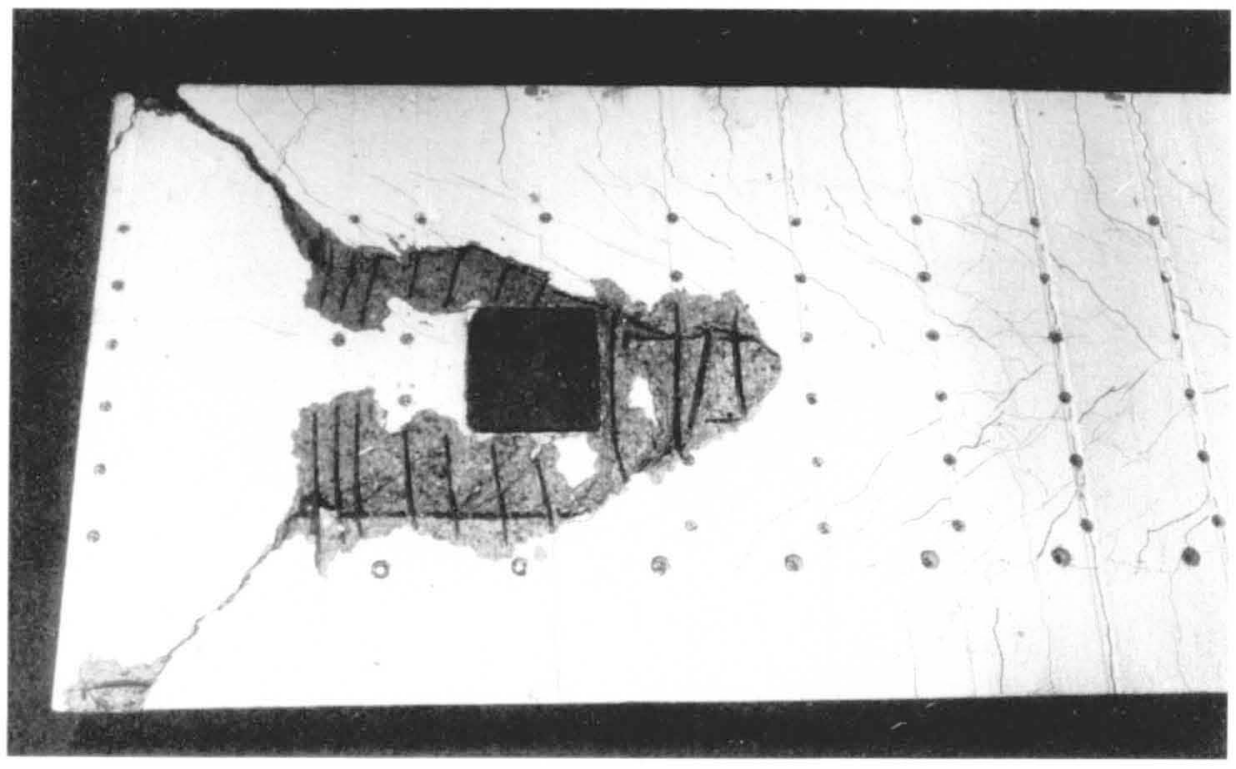

Ansicht von oben $\left(P_{u}=52 \dagger\right)$

Bild 41: Bruchbilder der Platte beim Träger Z 1 (linke Seite) 


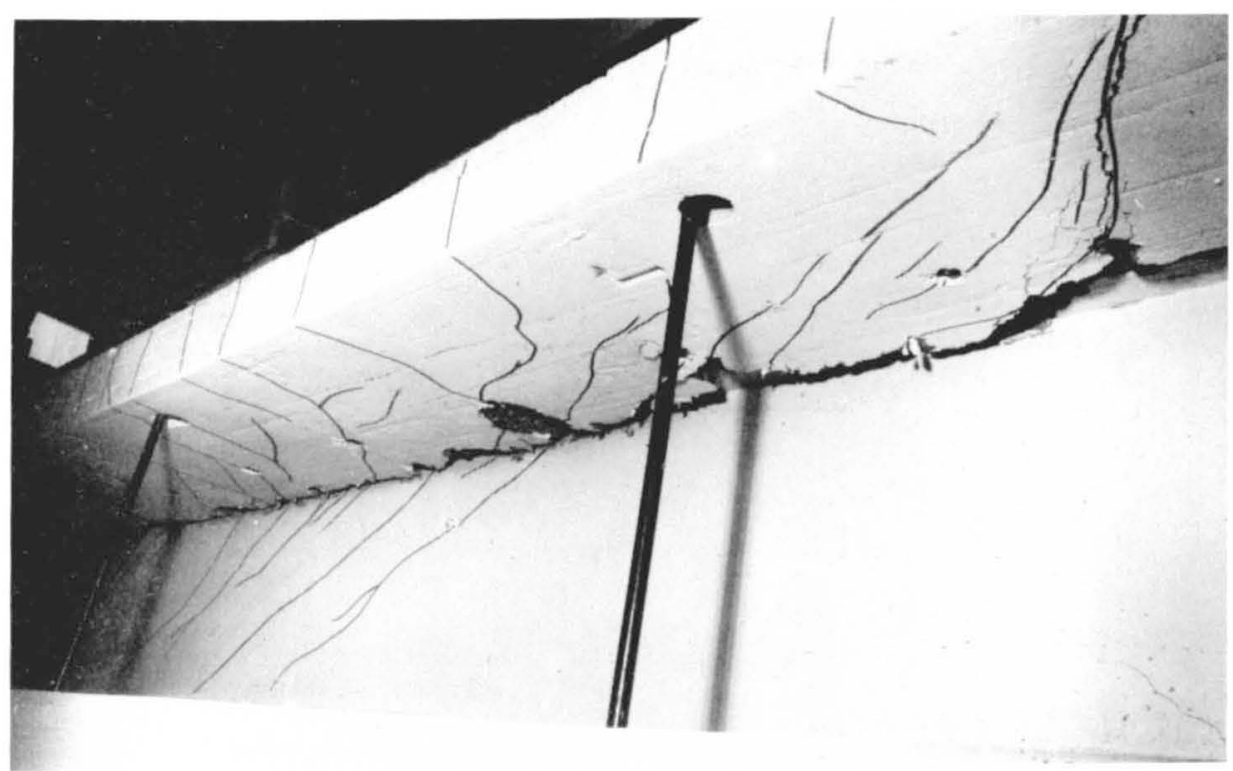

Ansicht von unten ( $\left.P_{\text {III }}=43 t, \bar{P}_{u} \leq 6.00 t\right)$

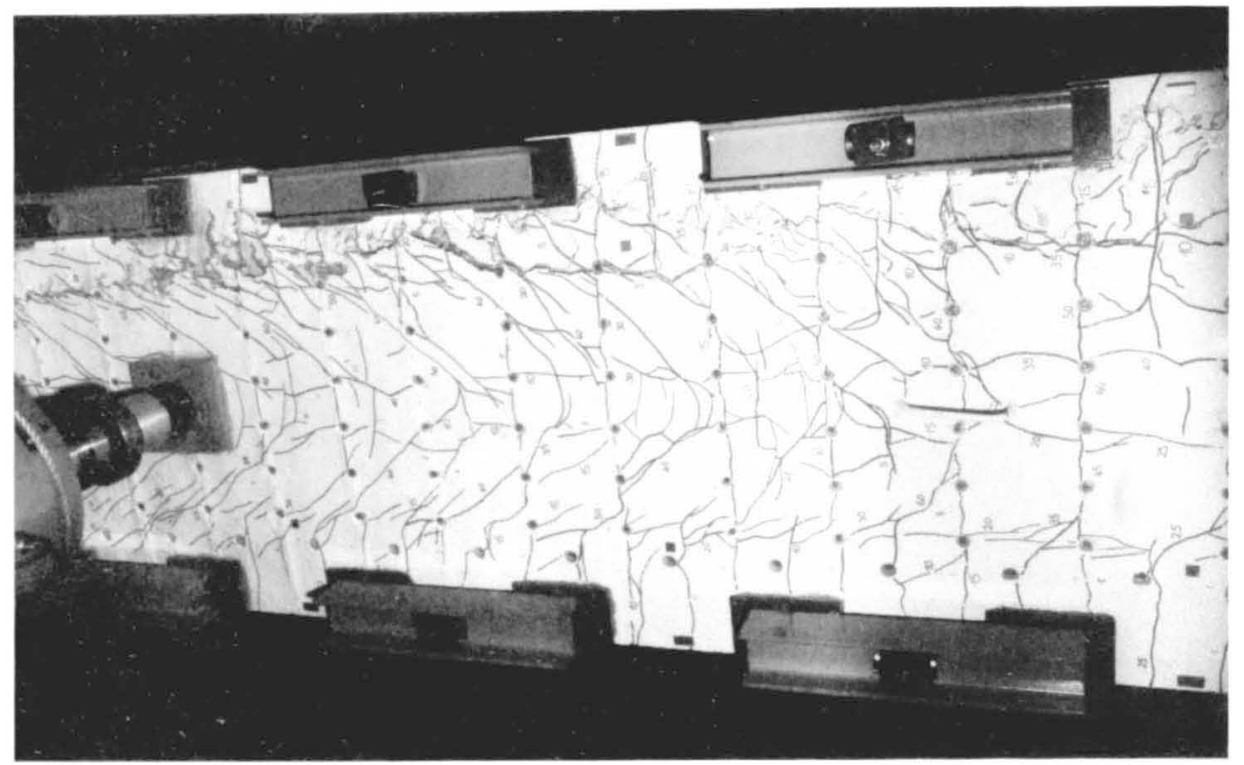

Ansicht von oben ( $P_{\text {III }}=43 t, \bar{P}_{u} \leq 6.00 t$ )

Bild 42: Bruchbilder der Platte beim Träger Z 4 (linke Seite) 


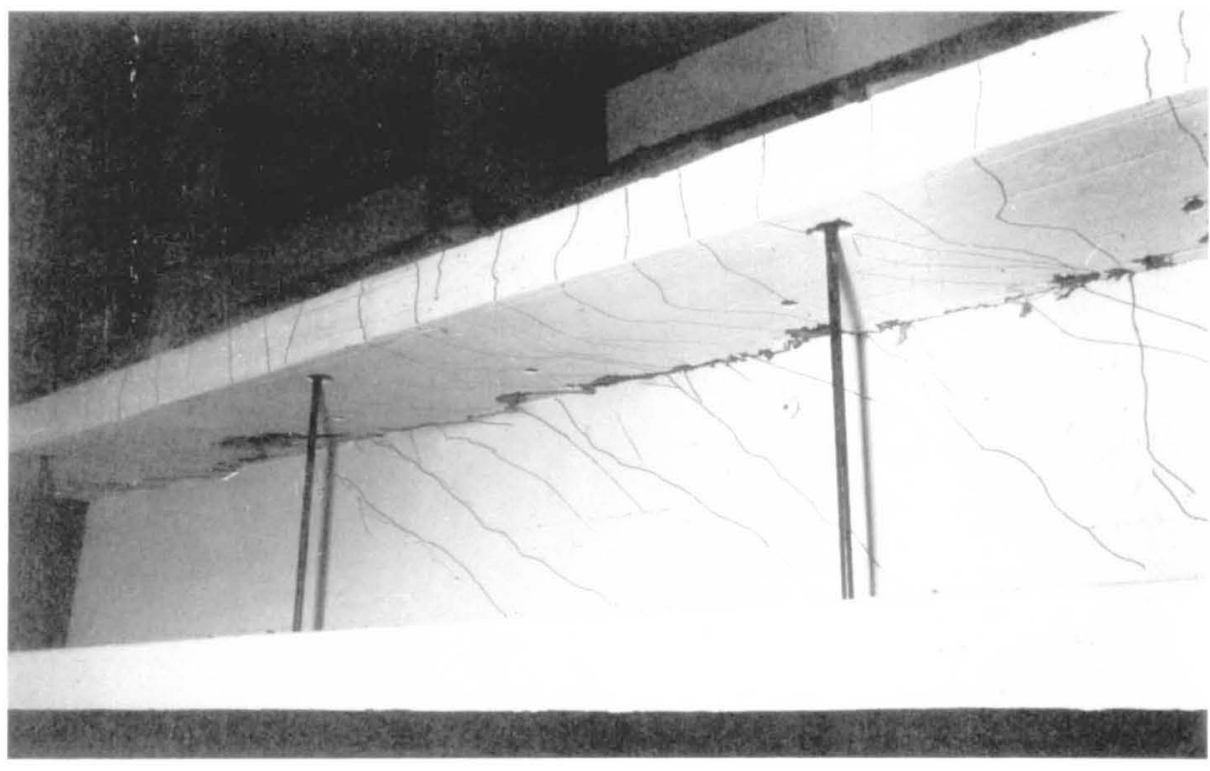

Ansicht von unten $\left(P_{u}=58 t\right)$

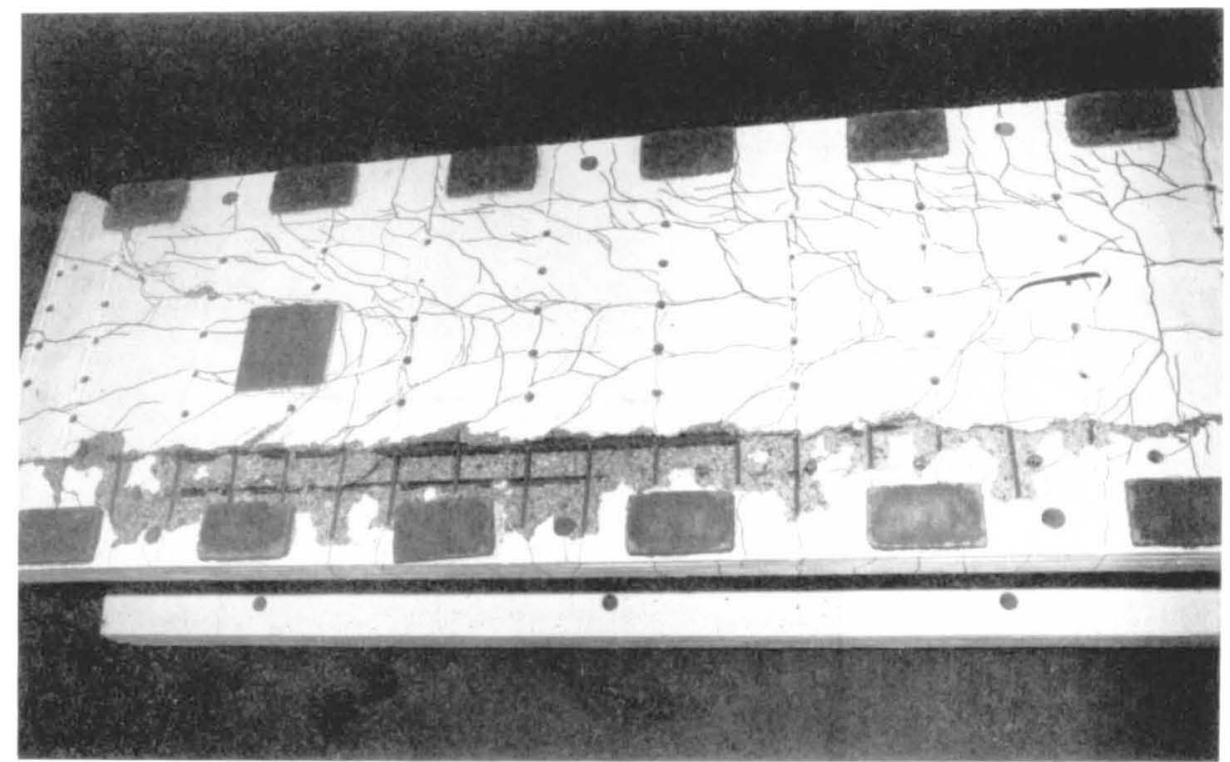

Ansicht von oben $\left(P_{u}=58 t\right)$

Bild 43: Bruchbilder der Platte beim Träger Z 3 (linke Seite) 


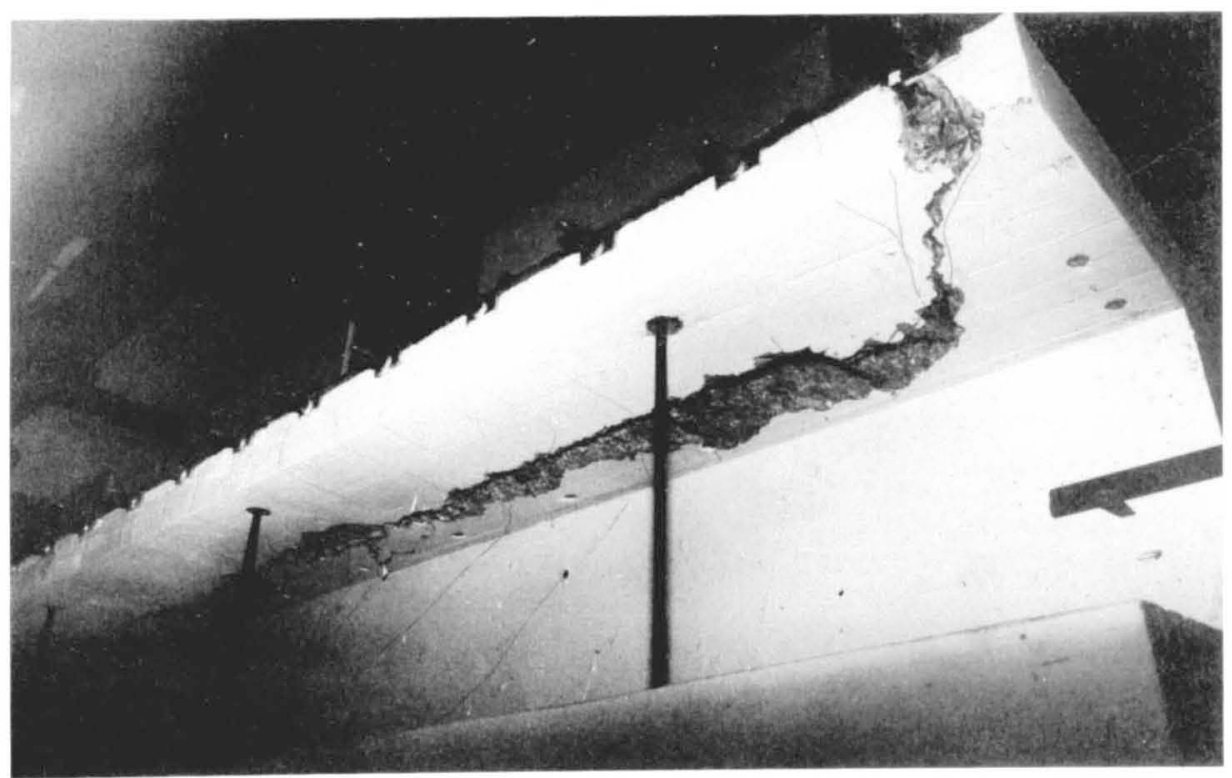

Ansicht von unten $\left(P_{u}=58 t\right)$

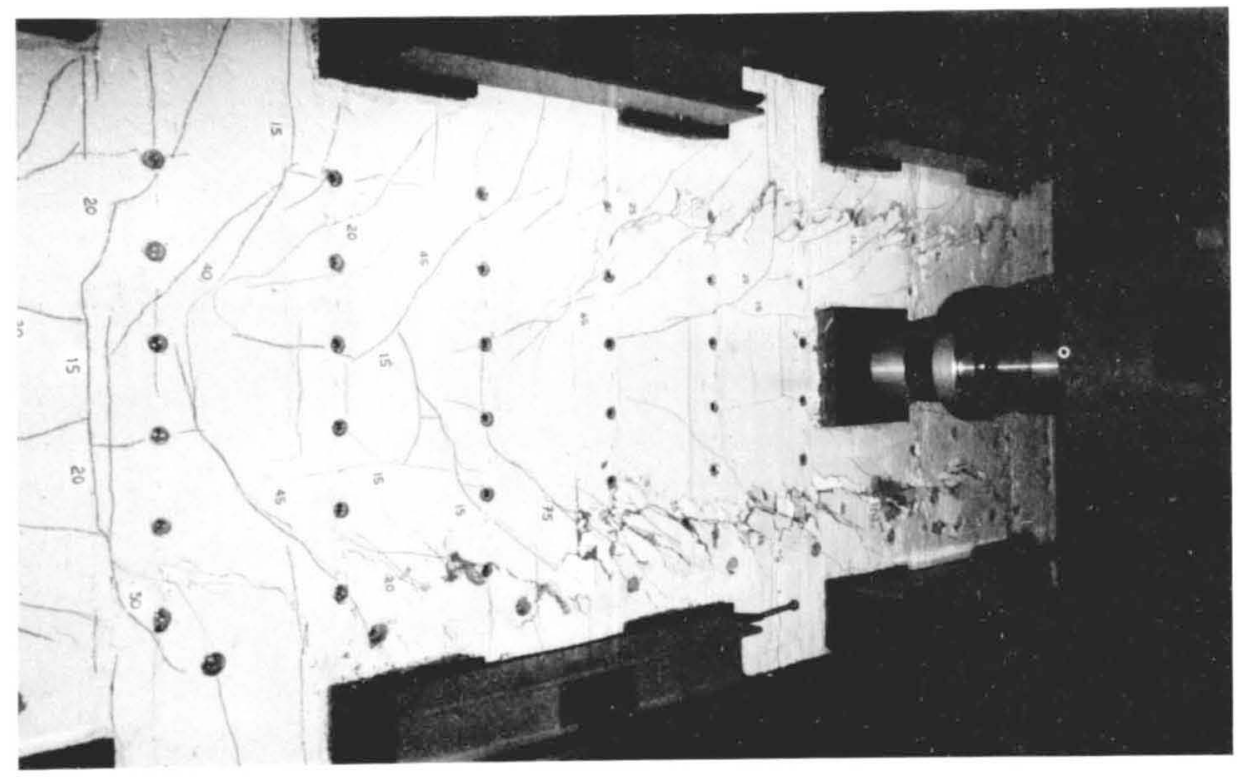

Ansicht von oben $\left(P_{u}=58 t\right)$

Bild 44: Bruchbilder der Platte beim Träger Z5 (rechte Seite) 


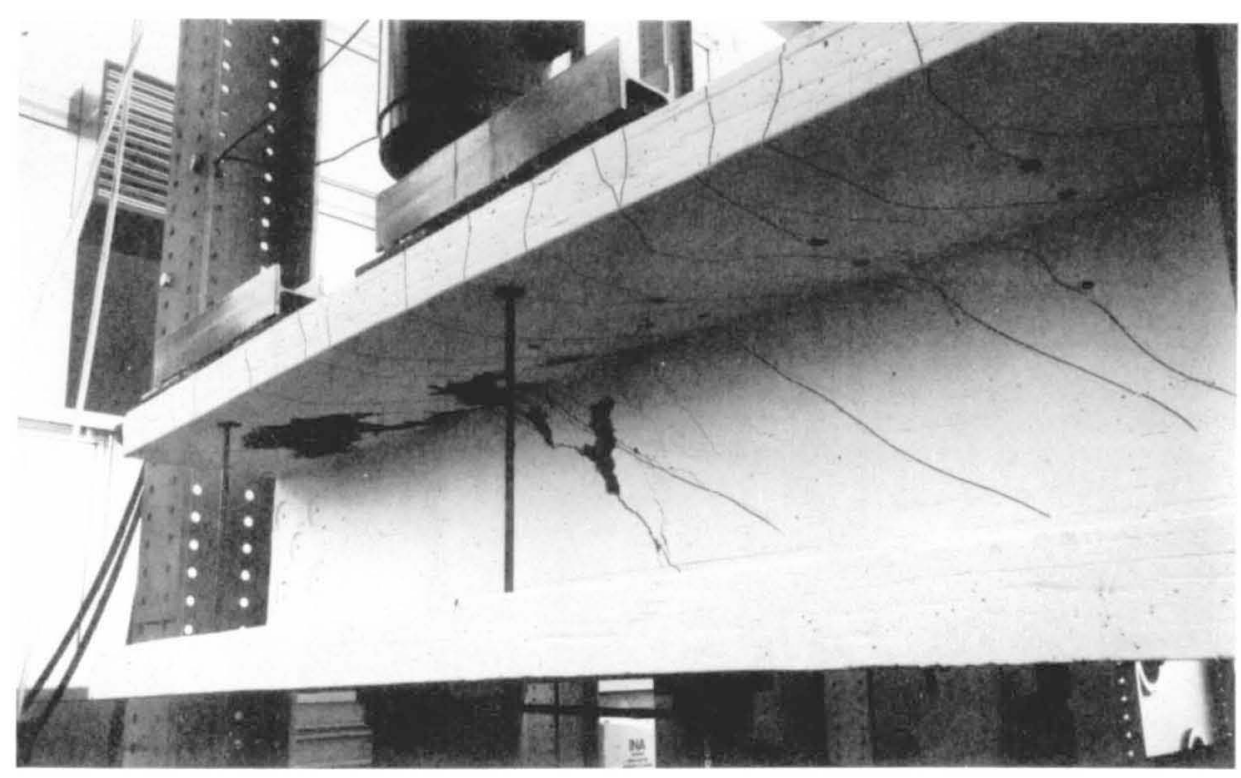

Ansicht von unten $\left(P_{u}=56 \uparrow\right)$

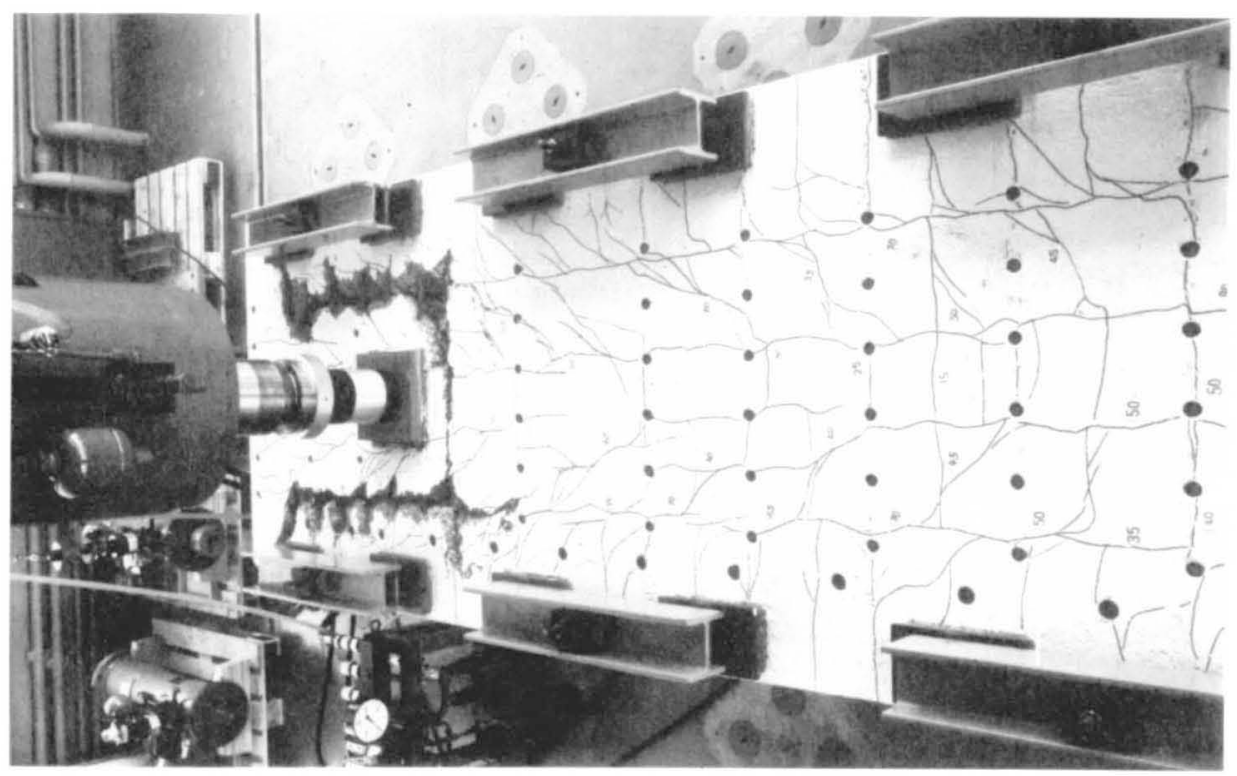

Ansicht von oben $\left(P_{u}=56 \uparrow\right)$

Bild 45: Bruchbilder der Platte beim Träger Z 6 (linke Seite) 

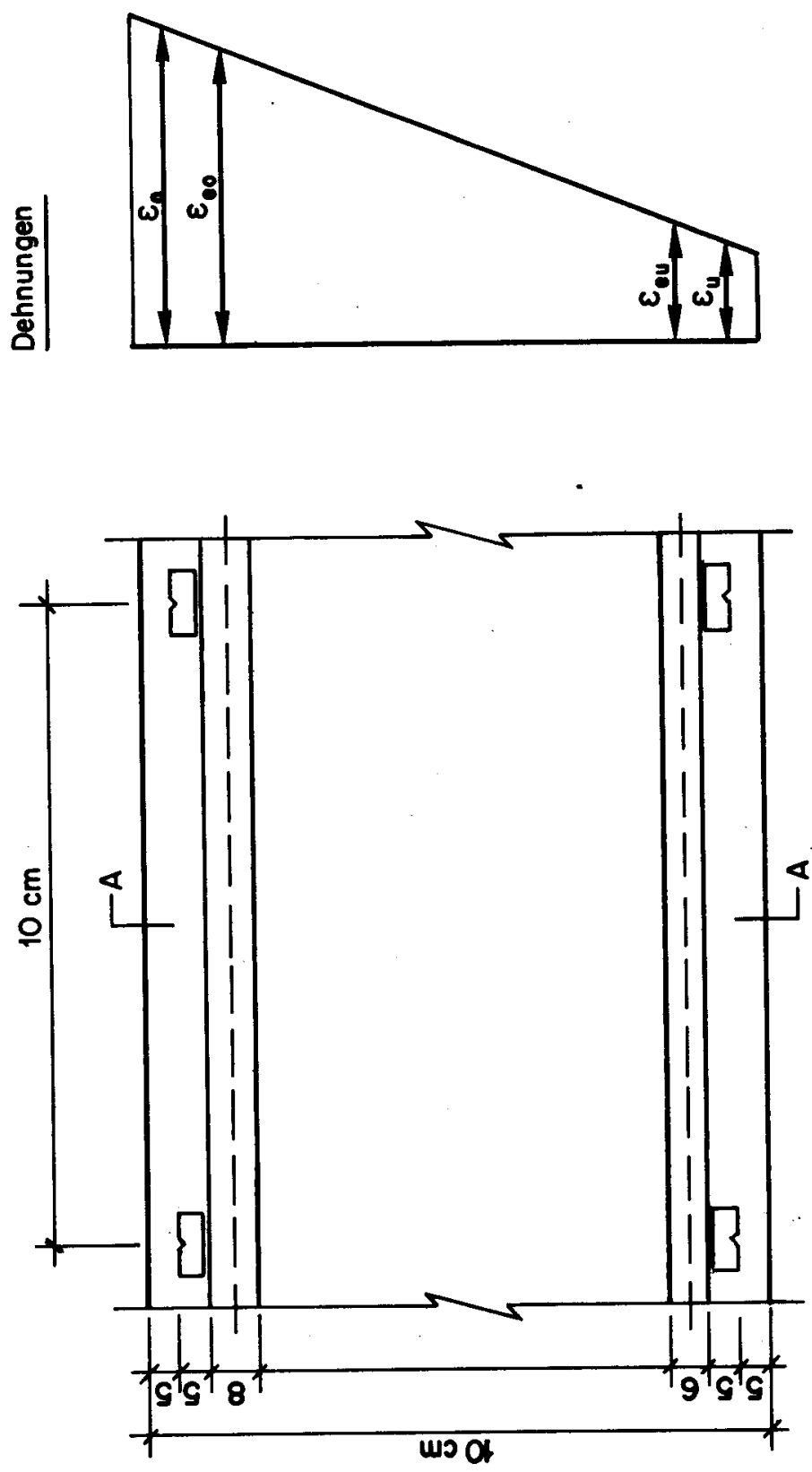

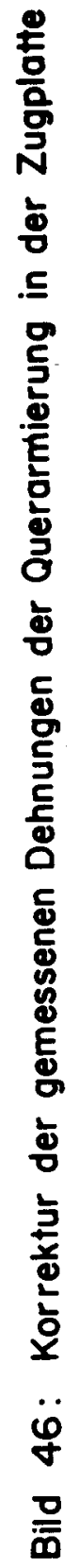




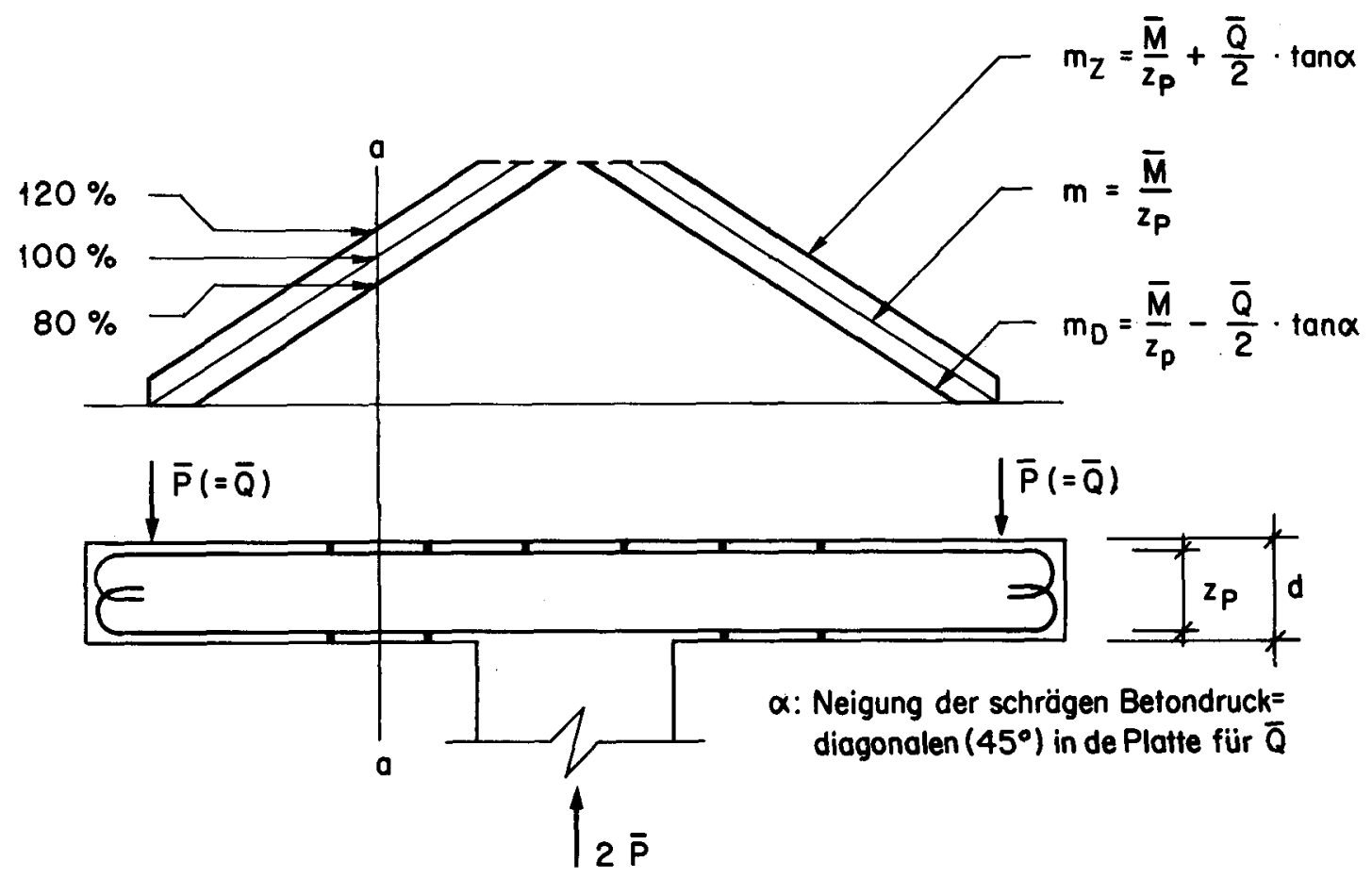

Bild 47: Einfluss des Querbiegemomentes $\bar{M}$ auf die Querzugkraft in der Plattenquerarmierung: $m_{Z}$ und $m_{D}$

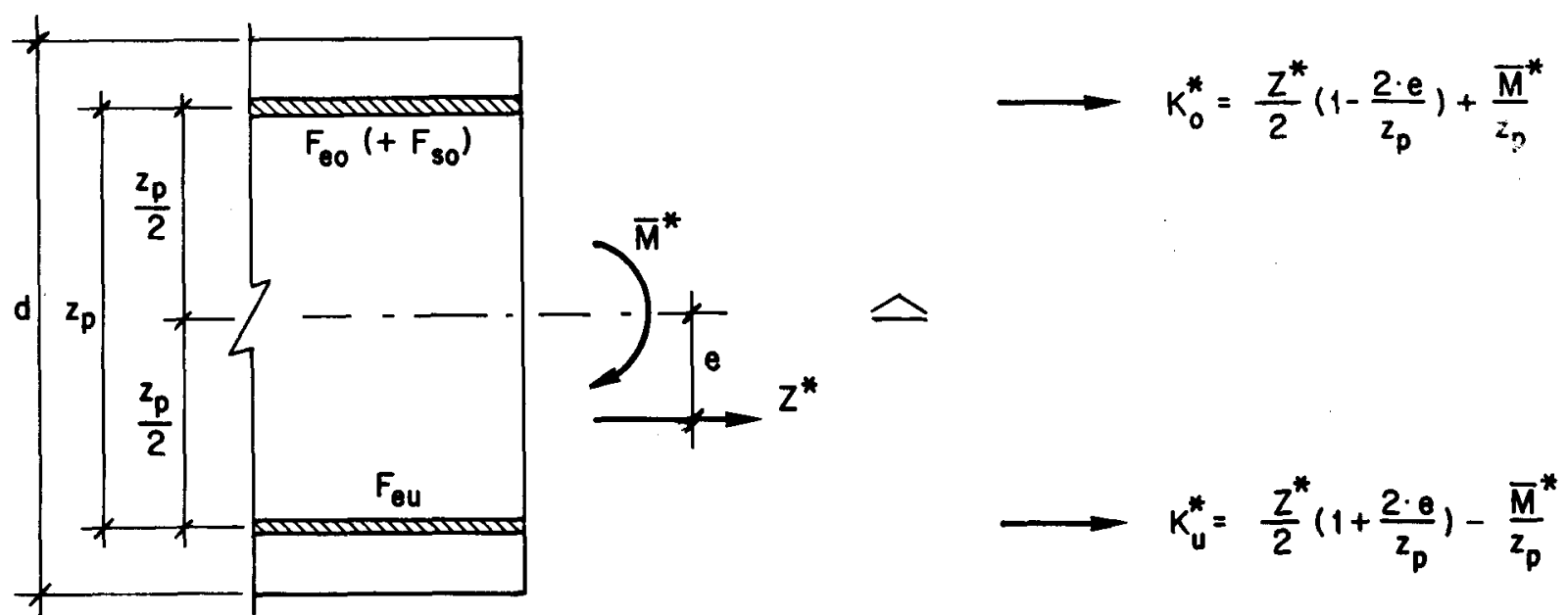

Bild 48: Bezeichnungen zu den Bemessungs - Empfehlungen 


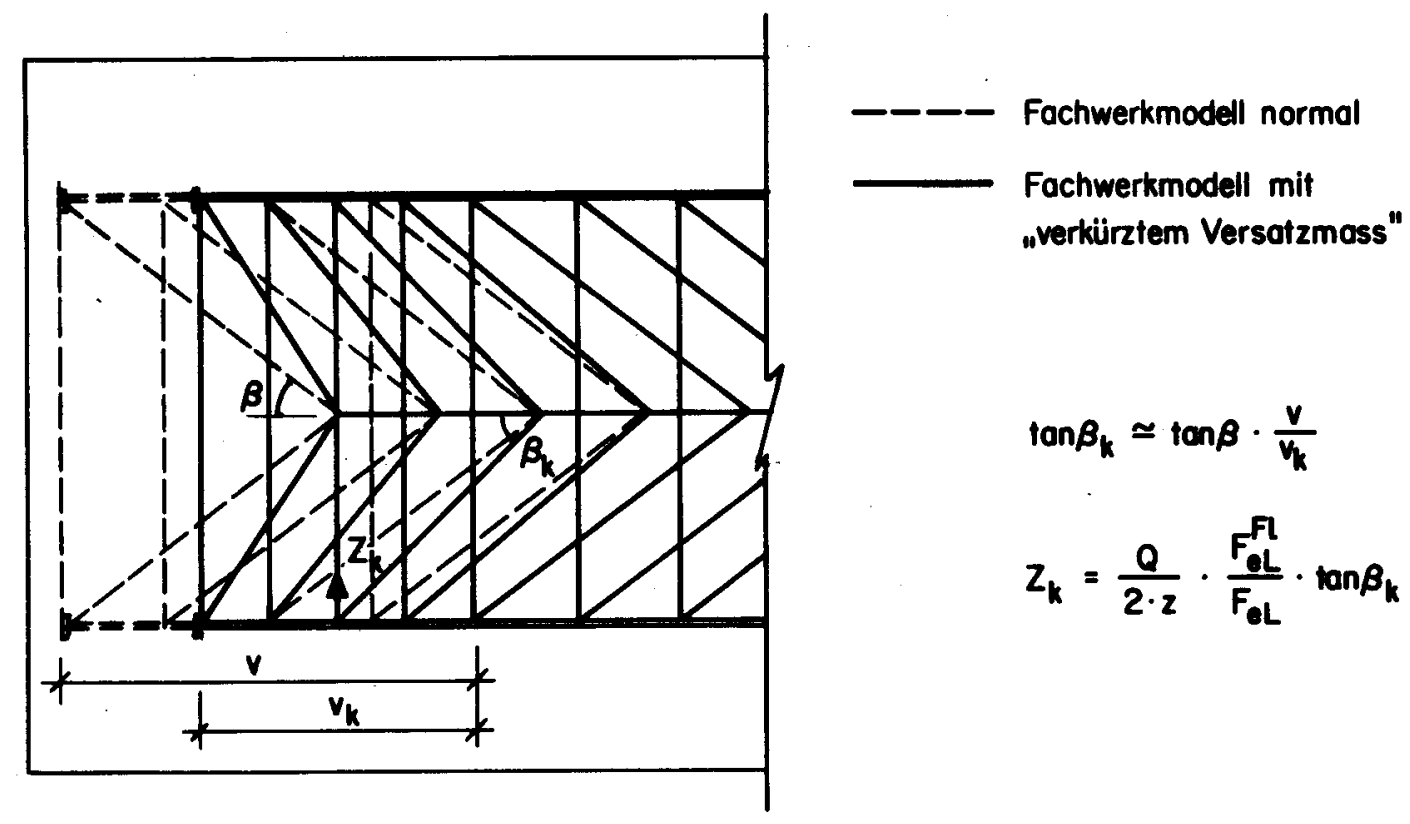

Bild 49: Flanschfachwerk beim "verkürzten Versatzmass" 
Träger Z 4:
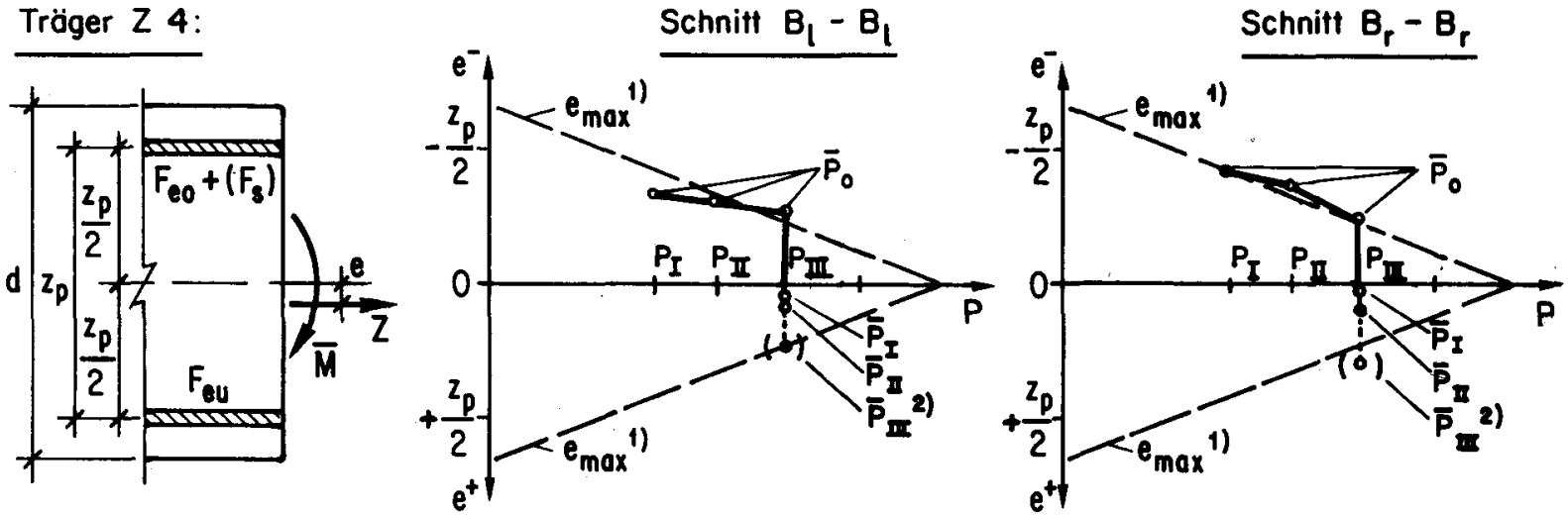

Träger Z 3 :
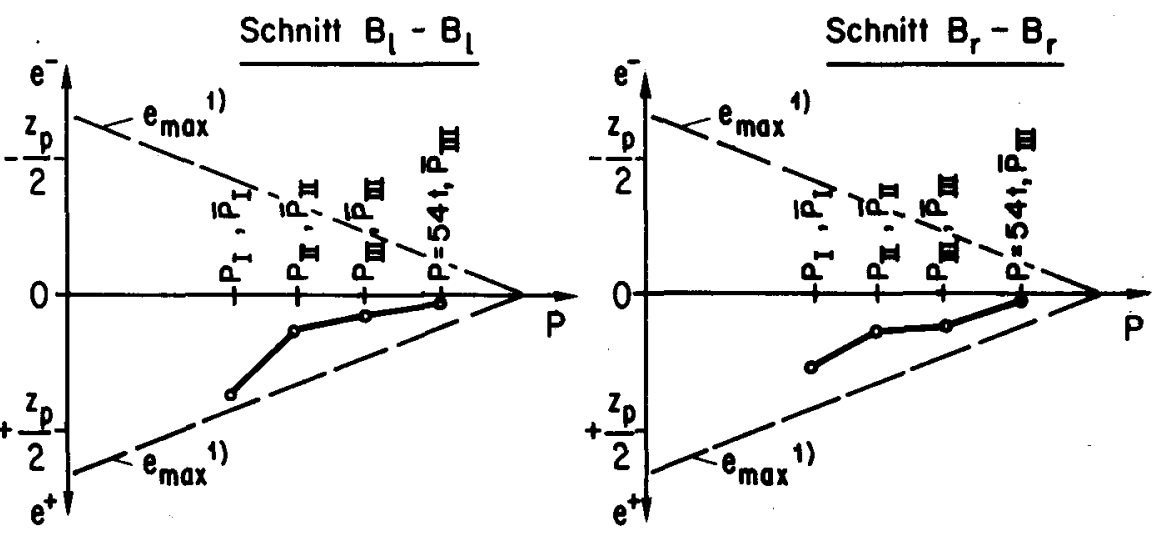

Träger Z 5:
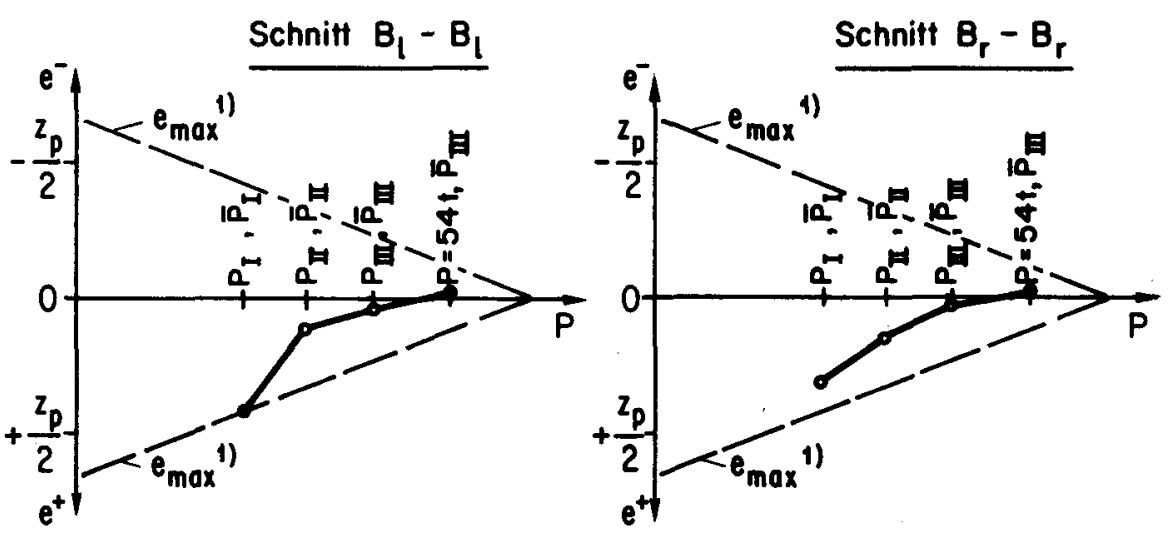

Träger Z 6 :
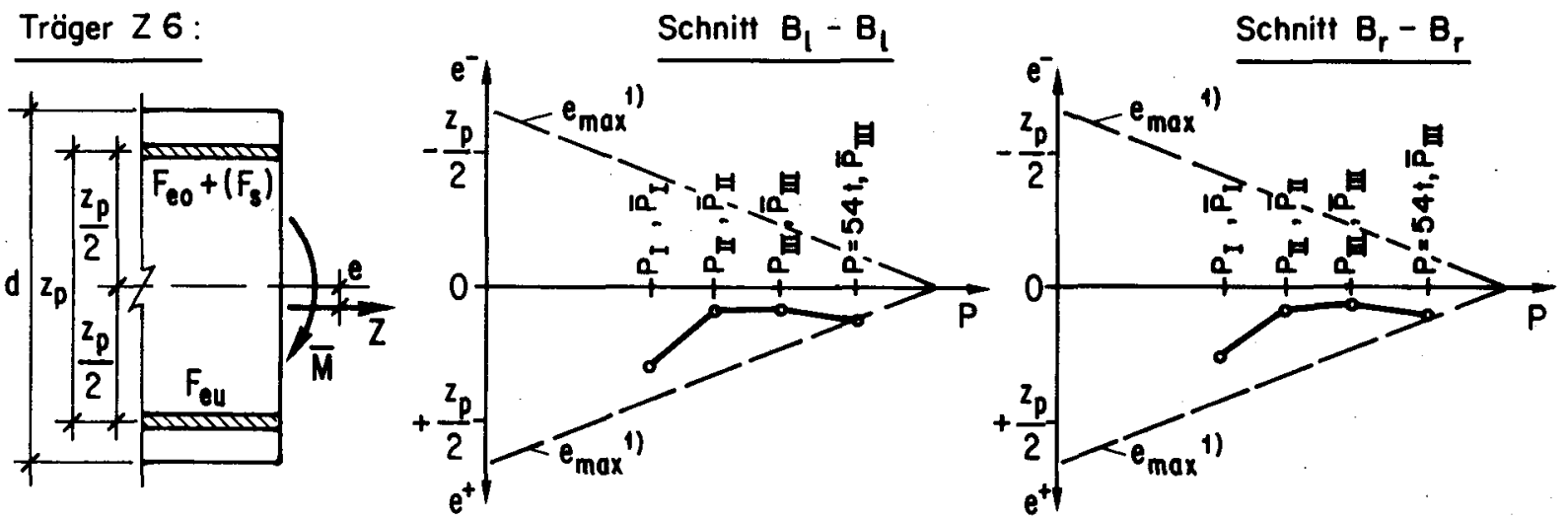

1) theoretischer Ausch noch GI (23)

2) extrapoliente Worte

Bild 50: Verlagerung des Angriffspunktes der Querzugkaft aus Löngsschub 
Querschnitt :

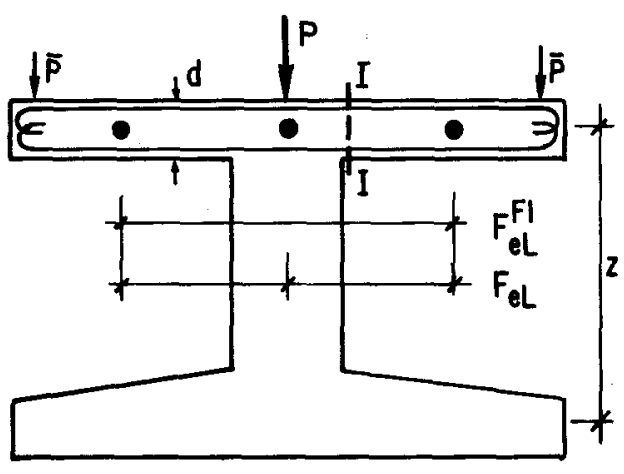

Flanschfochwerk :

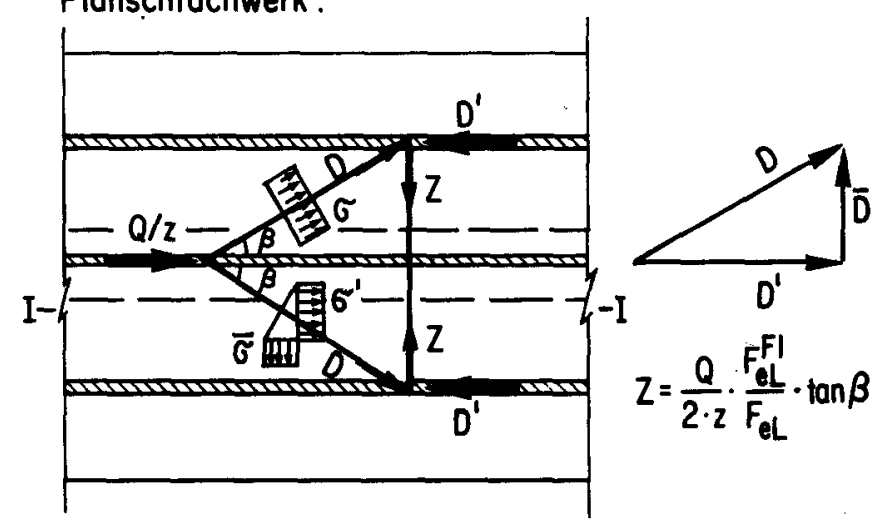

a) Längsschub allein, gleiche obere und untere Querarmierung

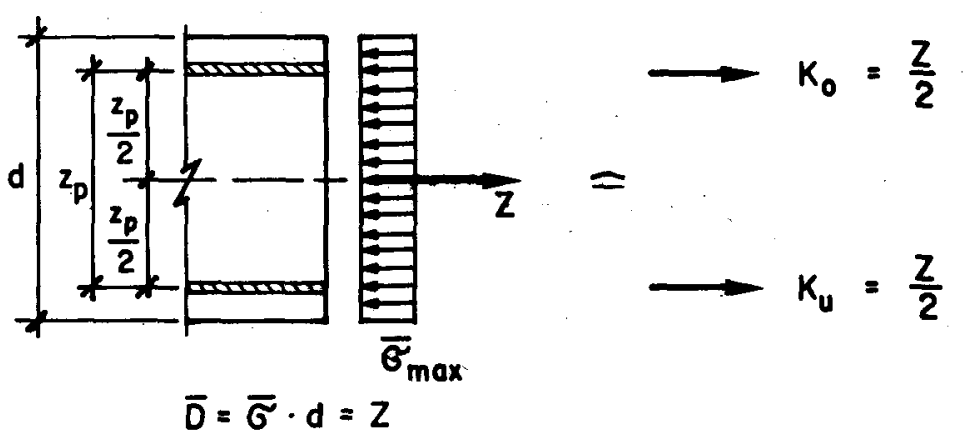

b) Löngsschub allein, ungleiche obere und untere Querarmierung

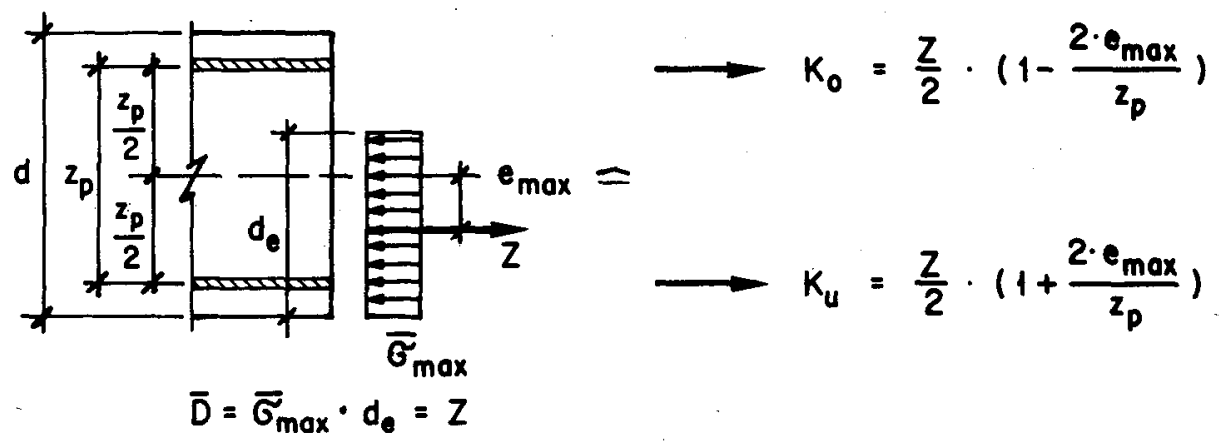

c) Längsschub mit Querbiegung, gleiche oder ungleiche obere und untere Querarmierung

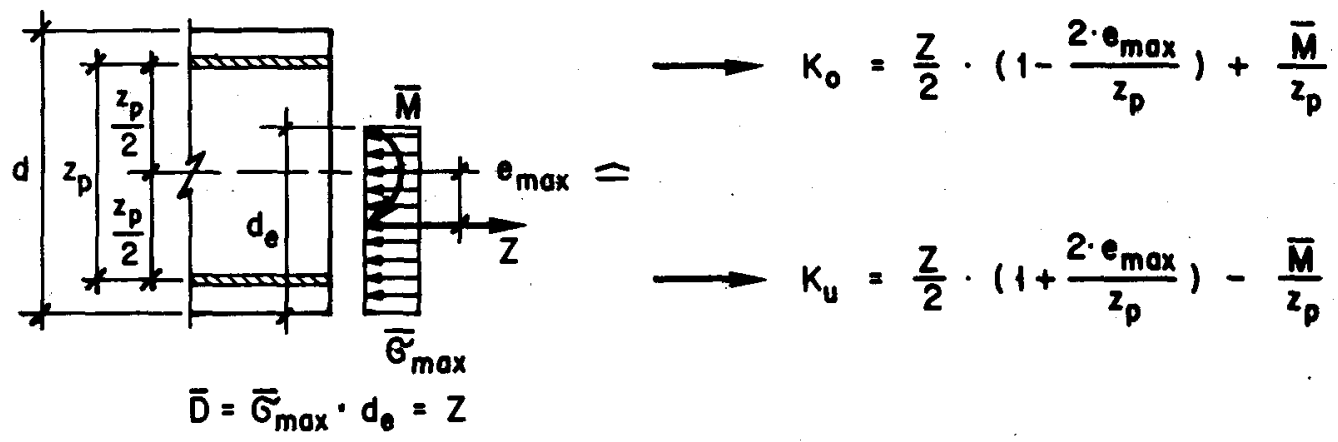

Bild 51: Gleichgewicht im Grenzzustand 

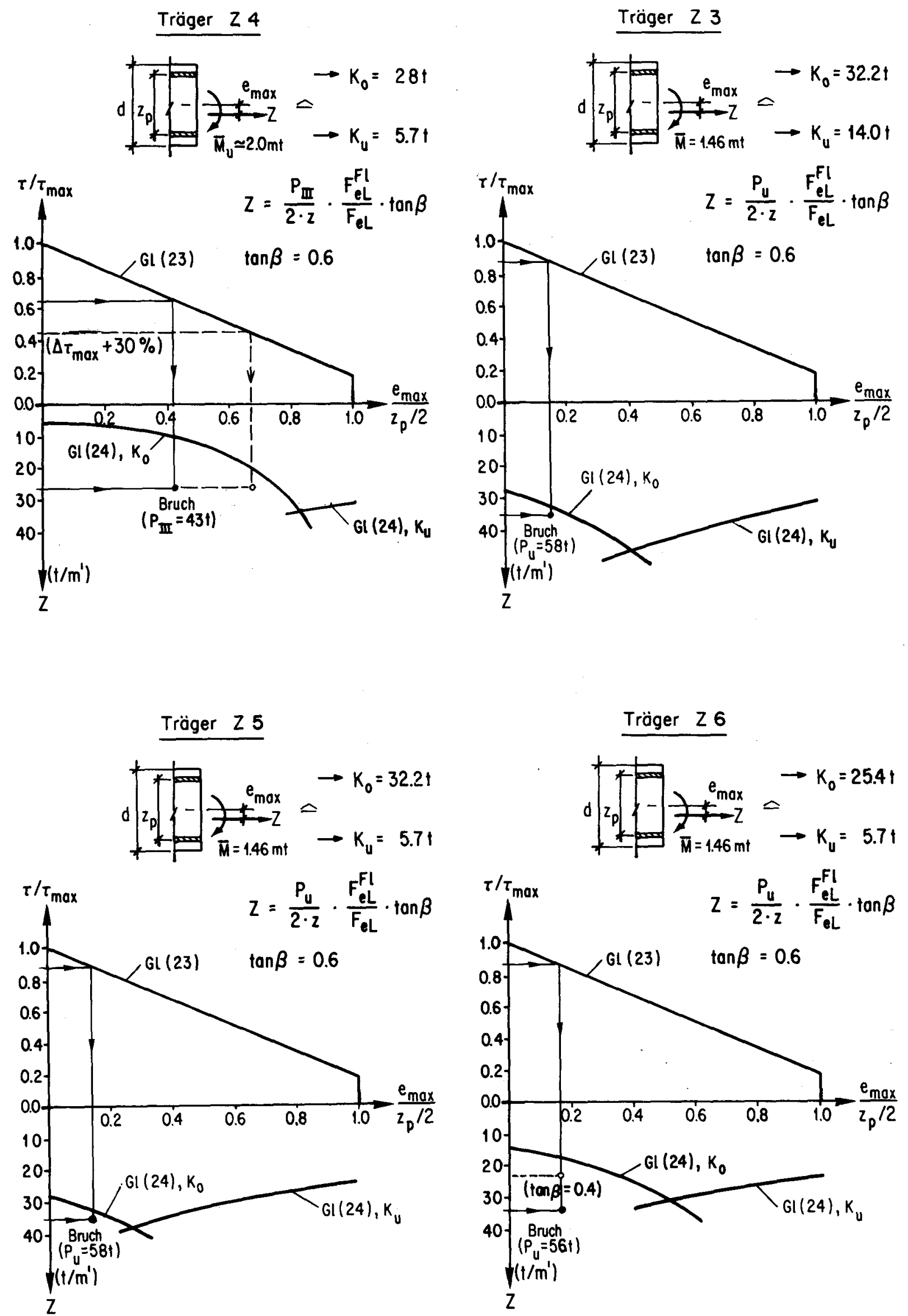

Bild 52: Vergleich mit Versuchsresultate 

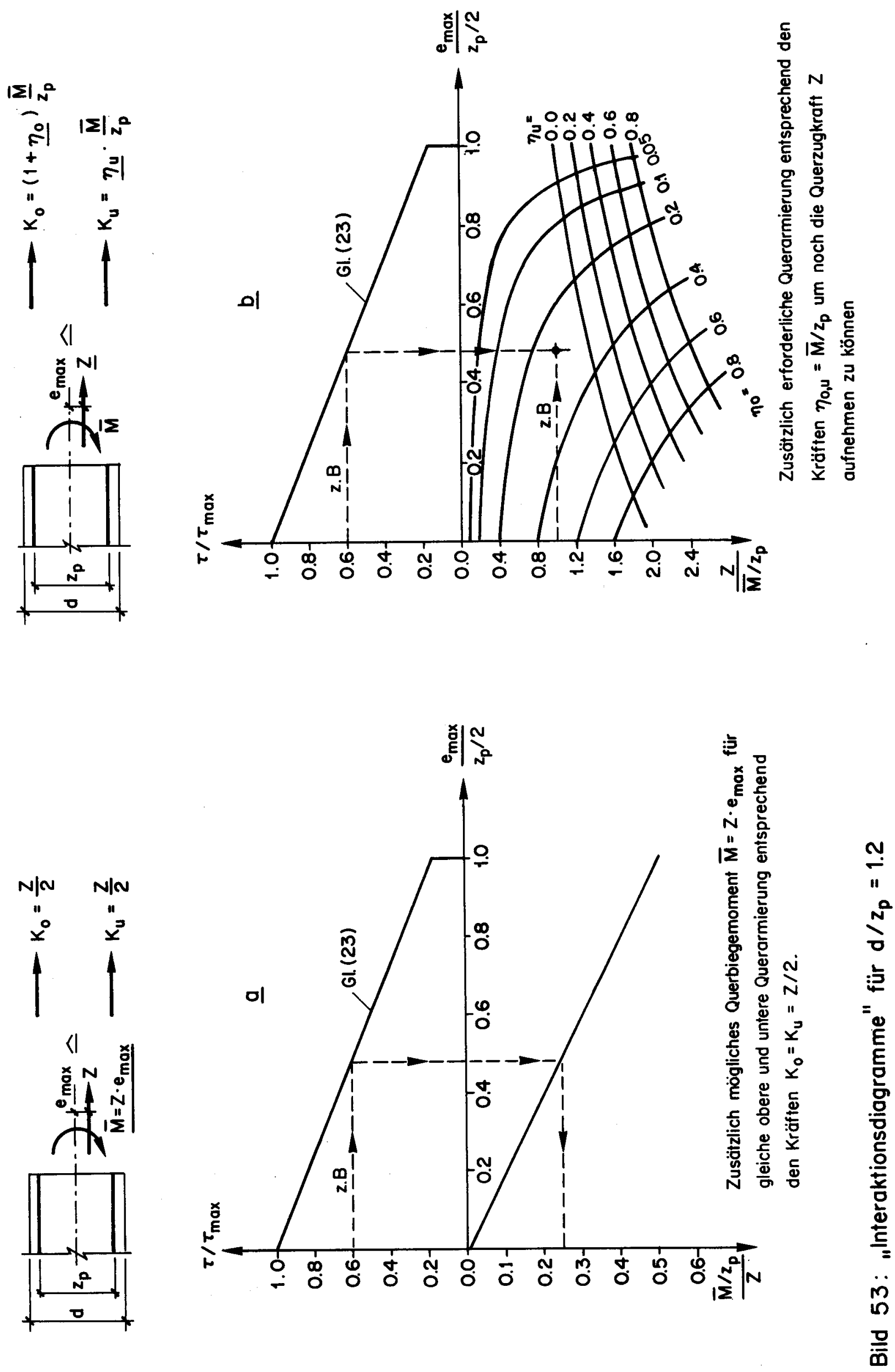


\section{Theoretischer Ansatz}

B. Thürlimann hat in [6] die plastische Analyse eines Fachwerk-Modells auf den Fall mit zusätzlicher Querbiegung erweitert. Sinngemäss wird hier für Längsschub allein sowie für Längsschub mit Querbiegung ein Varschlag für die bei einer Bemessung maximal anzusetzende Exzentrizität e der Querzugkraft aus Längsschub dargelegt (Bild 51).

\section{a) Längsschut al lein, gleiche obere und untere Querarmierung}

Im Schnitt I-I stehen (im Bruchzustand) die gleich grossen Fliesskräfte $K_{0}$, $K_{U}$ der oberen und unteren Querarmierung bzw. die zentrisch angreifende Querzugkraft $Z$ im Gleichgewicht mit der senkrecht zur Balkenaxe stehende Komponente $\bar{D}$ der schrägen Druckkraft D in der Diagonalen des Flanschfachwerkes (Bild 51a). Die Komponenten $\bar{\sigma}$ der Druckspannungen $\sigma$ sind gleichmässig über die Plattendicke d verteilt.

$$
\begin{aligned}
& \bar{D}=z=\frac{Q}{2 \cdot z} \cdot \frac{F_{e L}^{F l}}{F_{e L}} \cdot \tan \beta \wedge K_{0}+K_{U} \\
& \bar{\sigma}=\frac{\bar{D}}{d}=\tau \cdot \tan B
\end{aligned}
$$

\section{b) Längsschub allein, jedoch ungleiche obere und untere Querarmierung}

Im Schnitt I-I stehen (im Bruchzustand) die ungleich grossen Fliesskräfte $K_{0}$, $K_{U}$ der oberen und unteren Querarmierung bzw. die exzentrisch angreifende Querzugkraft $Z$ im Gleichgewicht mit der senkrecht zur Balkenaxe stehenden Komponenten $\bar{D}$ der schrägen Druckkraft $D$ in der Diagonalen des Flanschfachwerkes (Bild 51b). Wird näherungsweise eine gleichmässige Verteilung der Komponenten $\bar{\sigma}$ der Druckspannungen $\sigma$ angenommen, so erreicht die Exzentrizität e ihren Extremwert e max wenn die Spannungen $\sigma$ bzw. $\bar{\sigma}$ auf die Dicke de verteilt den Grenzwert $\sigma_{\max }$ bzw. $\bar{\sigma}_{\max }$ erreichen:

$$
d_{e} \cdot \sigma_{\max }=d \cdot \sigma ; d_{E} \cdot \bar{\sigma}_{\max }=d \cdot \bar{\sigma}
$$

In einem Versuch entspricht dieser Grenzwert der Stauchung des Betons im Druckfeld. In Bemessungsvorschriften werden die schrägen Betondruckspannungen durch das Einführen einer oberen Schubspannungsgrenze $\tau_{\max }$ begrenzt. Daher ist folgender Ansatz plausibel:

$$
\frac{\sigma}{\sigma_{\max }}=\frac{\bar{\sigma}}{\bar{\sigma}_{\max }}=\frac{\tau}{\tau_{\max }}
$$

Darin ist die Längsschubspannung

$$
\tau=\frac{Q}{2 \cdot d \cdot z} \cdot \frac{F l}{F L}
$$

Somit wird

$$
e_{\max }=\frac{d}{2}-\frac{d_{e}}{2}=\frac{d}{2}\left(1-\frac{\tau}{\tau_{\max }}\right)
$$


c) Längsschub mit Querbiegung, gleiche oder ungleiche obere und untere Querarmierung

Wird zum Längsschub zusätzlich ein Querbiegemoment $\bar{M}$ aufgebracht (Bild 51c), so werden die Kräfte $K_{0}$, $K_{u}$ in der oberen und unteren Querarmierung um die Querbiege-Zugkraft vergrössert bzw. um die Querbiege-Druckkraft verkleinert.

Im Schnitt I-I steht auch hier die exzentrisch angreifende Querzugkraft Z im Gleichgewicht mit der senkrecht zur Balkenaxe stehenden Komponenten $\bar{D}$ der schrägen Druckkraft $D$ in der Diagonalen des Flanschfachwerkes (Bild 51c). Wird näherungsweise eine gleichmässige Verteilung der Komponenten $\bar{\sigma}$ der Druckspannungen $\sigma$ angenommen, so erreicht die Exzentrizität e ihren Extremwert $e_{\max }$ wenn die Spannungen $\sigma$ bzw. $\bar{\sigma}$ auf die Dicke $d_{e}$ verteilt den Grenzwert $\sigma_{\max }$ bzw. $\bar{\sigma}_{\max }$ erreichen:

$$
d_{e} \cdot \sigma_{\max }=d \cdot \sigma ; d_{e} \cdot \bar{\sigma}_{\max }=d \cdot \bar{\sigma}
$$

In einem Versuch entspricht dieser Grenzwert der Stauchung des Betons im Druckfeld. In Bemessungsvorschriften werden die schrägen Betondruckspannungen durch das Einführen einer oberen Schubspannungsgrenze $\tau_{\max }$ begrenzt. Daher ist folgender Ansatz plausibel:

$$
\frac{\sigma}{\sigma_{\max }}=\frac{\bar{\sigma}}{\bar{\sigma}_{\max }}=\frac{\tau}{\tau_{\max }}
$$

Darin ist die Längsschubspannung

$$
\tau=\frac{Q}{2 \cdot d \cdot Z} \cdot \frac{\dot{F}_{\mathrm{EL}}^{F I}}{F_{\mathrm{eL}}}
$$

Somit wird

$$
e_{\text {max }}=\frac{d}{2}-\frac{d e}{2}=\frac{d}{2}\left(1-\frac{\tau}{\tau_{\max }}\right)
$$

Unter Berücksichtigung der maximal anzusetzenden Exzentrizität $e_{\max }$ der Querzugkraft kann die erforderliche Fliesskraft der Armierungen somit aus folgenden Formeln bestimmt werden :

bzw.

$$
k_{0, u}=\frac{z}{2}\left(1 \mp \frac{2 \cdot \varepsilon_{\max }}{z_{p}}\right) \pm \frac{\bar{M}}{z_{p}}
$$

$$
K_{0, u}=\frac{Z}{2}\left[\left(1 \mp \frac{d}{z_{p}}\left(1-\frac{\tau}{\tau_{\max }}\right)\right] \pm \frac{\bar{M}}{z_{p}}\right.
$$

Für $\tau=\tau_{\max }$ (bzw. für $e_{\max }=0$ ) vereinfacht sich die Gleichung (24) bzw. (25) zu

$$
k_{0, u}=\frac{Z}{2} \pm \frac{\bar{M}}{z_{p}}
$$




\section{Vergleich mit Versuchsresultaten}

Bild 52 zeigt den Vergleich zwischen der Querzugkraft $Z$ gemäss dem Flanschfachwerk nach Gl. (4) mit einem Ausbreitwinkel $\beta$ von $\tan \beta=0.6$ bei der Bruchlast und der Querzugkraft $Z$ aus Gl. (24) bzw. (25). Die obere Schubspannungsgrenze $\tau_{\max }$ wurde gemäss RL 34 der Norm SIA $162[5] \mathrm{zU} 6 \mathrm{t}_{1}^{*}=66 \mathrm{~kg} / \mathrm{cm}^{2}$ angesetzt.

Bei allen vier Trägern liegen die Versuchsresultate auf der sicheren Seite der Kurven entsprechend der Gl. (24) bzw. Gl. (25). Die relativ grosse Reserve bei Träger Z4 lässt auf ein Unterschätzen der oberen Schubspannungsgrenze $\tau_{\max }$ bzw. der maximal anzusetzenden Exzentrizität $e_{\max }$ schliessen. Wird die obere Schubspannungsgrenze um $\Delta \tau_{\max }=30 \%$ erhöht, so erzielt man eine bessere Uebereinstimmung (in Bild 51a eingetragen). Die relativ grosse Reserve beim Träger 26 kann mit der Verkleinerung des Ausbreitwinkels $\beta$ auf $\tan \beta<0.5$ ( $v g l$. Bild 30b) erklärt werden. Wird der Ausbreitwinkel $\beta$ auf $\tan \beta=0.4$ angesetzt, so erzielt man ebenfalls eine gute Uebereinstimmung (in Bild 51c eingetragen].

\section{Allgemeine Interaktions-Diagramme}

Das Bild 53 zeigt zwei aufgrund der Gleichungen (23) und (24) für d/z $=1.2$ dargestellte Interaktions-Diagramme.

a) Wird in einem Querschnitt oben und unten die gleiche Querarmierung für die Querzugkraft $Z$ eingelegt $\left(K_{0}=K_{U}=Z / 2\right)$, so kann aus Bild 53a herausgelesen werden, welches Querbiegemoment $\bar{M}=Z \cdot e_{\max }$ dieser Querschnitt noch aufnehmen kann.

b) Ist in einem Querschnitt vorerst nur Querarmierung für das Querbiegemoment $\bar{M}$ vorhanden (oben für $\bar{M} / z_{p}$; unten keine), so kann aus Bild 53b herausgelesen werden, wieviel zusätzliche Querarmierung entsprechend den Kräften $\eta_{0, u} \cdot \bar{M} / z_{p}$ dieser Querschnitt benötigt, um noch die Querzugkraft $z$ aufnehmen zu können.

Die Diagramme wurden wie folgt hergeleitet:

Aus G1. (23) ergibt sich

$$
\frac{e_{\max }}{z_{p} / 2}=\frac{d}{z_{p}}\left(1-\frac{\tau}{\tau_{\max }}\right)
$$

Aus G1. (24) wird für $k_{u}$

$$
\frac{z}{\bar{M} / z_{p}}=\left(1+\eta_{u}\right) \cdot \frac{2}{1+\frac{2 \cdot z_{\max }}{z_{p}}}
$$

Aus Gl. (24) wird für $K_{0}$

$$
\frac{z}{\bar{M} / z_{p}}=n_{0} \cdot \frac{2}{1-\frac{2 \cdot e_{\max }}{z_{p}}}
$$

